\title{
PATTERning STRATEgies IN NANOFABrication: From PERIOdic Patterns to Functional NANOSTRUCTURES
}


This research has been financially supported by the $\mathrm{MESA}^{+}$Institute for Nanotechnology and by NanoNed, a nanotechnology program of the Dutch Ministry of Economic Affairs. The research is part of the Strategic Research Orientations 'Advanced Photonic Structures' and 'Nanofabrication' and was carried out within the Supramolecular Chemistry \& Technology (SMCT) and Molecular Nanofabrication (MnF) groups, MESA+ Institute for Nanotechnology, University of Twente.

Cover design by Creative Obsessions - The photograph on the cover of this thesis is an artistic representation of my fingerprint in silicon and symbolizes my connections with the University of Twente.

Printing office: Digital Print Partners (DPP), Postbus 313, 3990 GC, Houten (http://www.gmgroep.nl/dpp)

(C) 2009 Christiaan M. Bruinink

Ph.D. thesis, University of Twente, Enschede, The Netherlands

No part of this work may be reproduced by print, photocopy or any other means without permission in writing from the author. 


\title{
PATterning STRATEgies In NANOFABrication: From PERIOdic Patterns to Functional NANOSTRUCTURES
}

\author{
PROEFSCHRIFT
}

\author{
ter verkrijging van \\ de graad van doctor aan de Universiteit Twente, \\ op gezag van de rector magnificus, \\ prof. dr. H. Brinksma, \\ volgens besluit van het College voor Promoties \\ in het openbaar te verdedigen \\ op donderdag 19 maart 2009 om 13:15 uur
} door

Christiaan Martijn Bruinink

geboren op 21 mei 1978 te Harderwijk 
Dit proefschrift is goedgekeurd door de promotoren:

prof. dr. ir. D. N. Reinhoudt

prof. dr. ir. J. Huskens 


\section{Contents}

Chapter 1 - General introduction 1

1.1 References 4

\section{Chapter 2 - Unconventional strategies in nanofabrication:} patterning principles

2.1 Introduction 7

2.2 Generation of patterns $\quad 10$

2.2.1 Pattern formation by size reduction $\quad 11$

2.2.1.1 Edge lithography 11

2.2.1.2 Additional strategies to size reduction 19

2.2.2 Pattern formation by writing 23

2.2.3 Pattern formation by self-assembly 26

2.2.3.1 Block copolymer lithography 26

2.2.3.2 Physical self-organization of polymers 29

2.2.3.3 Nanosphere lithography 31

2.2.4 Pattern formation by anodic oxidation (anodization) 32

2.2.5 Summary 34

2.3 Replication of patterns $\quad 35$

2.3.1 Pattern replication by imprinting 35

2.3.2 Pattern replication by conformal contact (soft lithography) 40

2.3.3 Summary 46

2.4 Conclusions $\quad 47$

2.5 References and notes $\quad 47$ 


\section{Chapter 3 - Supramolecular microcontact printing and dip-pen nanolithography on molecular printboards}

3.1 Introduction 60

3.2 Results and discussion $\quad 62$

3.2.1 Supramolecular microcontact printing - pattern stability 63

3.2.2 Electroless deposition - fabrication of metal patterns on the printboard 69

3.2.3 Supramolecular DPN - writing local molecular patterns at the sub-100 nm scale $\quad 70$

3.3 Conclusions 72

3.4 Experimental $\quad 72$

\begin{tabular}{ll}
3.5 References and notes & 77 \\
\hline
\end{tabular}

Chapter 4 - Stamps for high-resolution soft lithography

4.1 Introduction

4.2 Results and discussion

4.2.1 General procedure for the fabrication of high-resolution polymer templates

4.2.2 Fabrication of second-generation PDMS stamps

4.2.3 Application of second-generation stamps in soft lithography and the selective etching of gold

4.2.4 Pattern transfer by reactive ion etching

4.3 Conclusions 92

4.4 Experimental 93

4.5 References and notes 95 


\section{Chapter 5 - Fabrication of functional polymer templates as}

5.1 Introduction 97

5.2 Results and discussion 100

5.2.1 CFL templates as high-resolution molds for NIL 100

5.2.1.1 UV crosslinking for post-processing stabilization 101

5.2.1.2 Thermal imprinting and pattern transfer 103

5.2.2 CFL templates as etch masks in wet-chemical etching of metals 106

5.2.2.1 Effect of PDMS transfer on the template processability 107

5.2.2.2 Effect of over-etching on the feature dimensions

5.3 Conclusions 112

5.4 Experimental 113

5.5 References and notes 116

\section{Chapter 6 - Accurate pattern inversion and pattern transfer techniques for nanolithography}

6.1 Introduction

6.2.1 LOCOS for pattern inversion into thermal $\mathrm{SiO}_{2}$

6.2.1.1 Design rules for the transfer layers

6.2.1.2 Pattern inversion of resist patterns into thermal $\mathrm{SiO}_{2}$

6.2.2 Profile control by cryogenic etching

6.3 Conclusions 


\section{Chapter 7 - Fabrication, replication and characterization of 2D photonic crystal waveguides}

7.1 Introduction

7.2 Results and discussion 139

7.2.1 Structural design of the 2D PhCWG

7.2.2 Fabrication of the nanoimprint mold

7.2.3 Replication of 2D PhCWGs

7.2.3.1 Thermal nanoimprint lithography

7.2.3.2 LOCOS inversion procedure

7.2.3.3 Anisotropic etching of waveguide structures into SOI

7.2.4 Optical characterization by PSTM

7.2.4.1 Amplitude analysis of the 2D PhCWG

7.2.4.2 Band structure analysis of the $2 \mathrm{D}$ PhCWG

7.3 Conclusions 


\section{General introduction}

Nanofabrication is central to the development of several emerging technologies by facilitating the structuring of matter in the $1-100 \mathrm{~nm}$ regime. From a technological standpoint, the ability to fabricate structures at these spatial dimensions guarantees the essential continuation in the miniaturization of functional devices, also known in integrated circuit (IC) manufacturing as Moore's Law. ${ }^{[1]}$ The advantages of generating smaller devices are numerous, including lower costs, lower power consumption and higher performance. From a scientific standpoint, nanofabrication also allows for the exploration of fascinating (and in some cases technologically important) occurrences 
that only occur at nanometer dimensions. Revolutionary devices including quantum dot lasers, ${ }^{[2]}$ quantum cascade lasers, ${ }^{[3]}$ and single-electron transistors ${ }^{[4]}$ are the outcome of fundamental studies on electronic processes as the quantum size effect (QSE) ${ }^{[5]}$ and single-electron tunneling (SET). ${ }^{[6]}$

The drive towards the miniaturization of electronic circuits comes at the penalty of higher resistances and higher levels of power dissipation. The advantages of using light instead of electrons as the information carrier account for the efforts that are undertaken to progress the high-density integration and system performance towards all-optical circuits by the incorporation of photonic crystal $(\mathrm{PhC})$ structures. ${ }^{[7]} \mathrm{PhC}$ structures represent a novel class of optical materials (also present in nature ${ }^{[8]}$ ) that contain periodic modulations in dielectric contrast resulting from two ${ }^{-[9]}$ and/or threedimensional ${ }^{[10]}$ structuring of matter at the scale of the optical wavelength. The resulting characteristic photonic band gap $(\mathrm{PBG})$ for a specific range of frequencies ${ }^{[11]}$ is responsible for the corresponding strong confinement and localization of light, ${ }^{[12]}$ and for the control ${ }^{[13]}$ and redistribution ${ }^{[14]}$ of spontaneous emission of light inside the $\mathrm{PhC}$ structures. These are essential elements in the development of future all-optical circuits. Several examples of devices that illustrate the full potential of $\mathrm{PhCs}$ include lossless waveguides, ${ }^{[9]}$ zero-threshold lasers $^{[15]}$ and photonic crystal fibers. ${ }^{[16,17]}$

The major driving force for these technological developments is the demand for nanofabrication techniques that are capable of generating sub-100 nm features. Several state-of-the-art techniques such as deep UV and extreme UV photolithography, electron-beam writing, and ion-beam lithography, are capable of generating nanostructures. However, research and development of new (unconventional) fabrication strategies ${ }^{[18]}$ is crucial despite the enormous success and continuous efforts to extend the fabrication capabilities of these conventional techniques in terms of costeffectiveness and high-throughput processing of sub-100 nm structures. This thesis contributes to the continuous development of unconventional fabrication strategies in several areas of nanofabrication.

Chapter 2 presents a procedural review of unconventional nanofabrication for lowcost and fast prototyping of nanostructures in the sub-100 nm range (instead of dealing with extensions of conventional nanofabrication). Of particular interest are potential patterning principles that result in significant advancements in terms of 
resolution, reproducibility and uniformity of the structures. Many examples of the fabrication of periodic and functional nanostructures are given in this chapter, with a preference for the fabrication of potential nanostructures in photonics.

The first part of this thesis (Chapters 3, 4 and 5) deals with several interesting patterning strategies of matter (molecules and polymers). The main purpose of this research is to investigate in detail the characteristics of supramolecular patterning of molecules and capillary force patterning of polymers in terms of resolution, reproducibility and uniformity by patterning periodic patterns on surfaces.

Chapter 3 describes a patterning strategy by using multiple supramolecular interactions in the transfer of high-stability patterns of functional molecules at a lateral resolution of $60 \mathrm{~nm}$ onto molecular printboards. Printing and competitive rinsing experiments have been carried out to assess the influence of the valency of the guest molecules on the pattern stability and to illustrate the concept of supramolecular microcontact printing $(\mu \mathrm{CP})$ and dip-pen nanolithography (DPN) on molecular printboards. Additionally, the molecular patterns of dendrimer-stabilized nanoparticles have been further exploited as catalysts in the electroless deposition of metals on top of printboards.

The next two chapters (Chapters 4 and 5) discuss a facile fabrication strategy for the high-resolution patterning of polymer films by capillary force lithography (CFL). Additionally to investigate the characteristics of capillary rise in edge lithography, the resulting CFL patterns have been exploited (for the first time) as functional templates in high-resolution lithography. Structural analysis has been carried out by atomic force microscopy (AFM) and scanning electron microscopy (SEM) to assess the mechanical stability and integrity of CFL templates and the corresponding derivatives (in the form of masters, molds and etch masks) during processing.

Chapter 4 illustrates the potential of these templates as masters in the fabrication of second-generation stamps for application in high-resolution soft lithography. This has been shown by several different $\mu \mathrm{CP}$ experiments on gold and subsequent wetchemical etching in the generation of metal patterns on silicon substrates. Structural analysis has been carried out to investigate the discrepancy between the lateral dimensions of the metal features and the indentations present on the secondgeneration stamps. Additionally, these metal patterns have been exploited as etch 
masks in the generation of nanochannels into silicon substrates by reactive ion etching (RIE).

Chapter 5 describes two potential extensions of the use of CFL templates as masters in nanoimprint lithography (NIL) and as etch masks in the wet-chemical etching of metals by incorporating different polymers into the CFL procedure. Transfer of stamp residues has been shown by X-ray photoelectron spectroscopy (XPS) to occur during CFL processing, and corresponding measures have been undertaken to increase the processability of the CFL templates. Finally, the basic concept of overetching is shown as a strategy to feature reduction.

The second part of this thesis (Chapters 6 and 7) illustrates a novel strategy for the accurate pattern inversion of polymer patterns into silicon oxide $\left(\mathrm{SiO}_{2}\right)$ by incorporation of local oxidation of silicon (LOCOS) into NIL processing. The final goal of this research has been to develop a low-cost replication procedure of twodimensional photonic crystal waveguides (2D PhCWGs).

Chapter 6 describes the entire engineering technology to set the design rules for optimal LOCOS inversion in terms of layer thickness and etch selectivities. The partial conversion of silicon nitride $\left(\mathrm{Si}_{3} \mathrm{~N}_{4}\right)$ to silicon oxynitride $\left(\mathrm{SiN}_{\mathrm{x}} \mathrm{O}_{\mathrm{y}}\right)$ during the oxidation step of the LOCOS procedure is shown by etch rate measurements. The use of guidelines is shown to systematically locate the optimal parameters for silicon etching at high anisotropy and high etch selectivity using ultrathin $\mathrm{SiO}_{2}$ etch masks.

Chapter 7 presents the successful fabrication and replication of 2D PhCWGs on silicon-on-insulator (SOI) substrates by elaborating on the technological advancements in NIL processing set in Chapter 6. Local examination using photon scanning tunneling microscopy (PSTM) has been carried out to assess the guiding properties of the resulting $2 \mathrm{D}$ PhCWGs (in terms of confinement and loss of the propagating light) at wavelengths in the telecommunication range.

\subsection{References}

[2] S. Fafard, K. Hinzer, S. Raymond, M. Dion, J. McCaffrey, Y. Feng, S. Charbonneau, Science 1996, 274, 1350. 
[3] J. Faist, F. Capasso, D. L. Sivco, C. Sirtori, A. L. Hutchinson, A. Y. Cho, Science 1994, 264, 553.

[4] M. H. Devoret, D. Esteve, C. Urbina, Nature 1992, 360, 547.

[5] M. Sundaram, S. A. Chalmers, P. F. Hopkins, A. C. Gossard, Science 1991, 254, 1326.

[6] M. H. Devoret, D. Esteve, C. Urbina, Nature 1992, 360, 547.

[7] J. D. Joannopoulos, P. R. Villeneuve, S. Fan, Nature 1997, 386, 143.

[8] P. Vukusic, J. R. Sambles, Nature 2003, 424, 852.

[9] Examples of 2D photonic crystal waveguides: (a) T. F. Krauss, R. M. De La Rue, S. Brand, Nature 1996, 383, 699. (b) E. Chow, S. Y. Lin, S. G. Johnson, P. R. Villeneuve, J. D. Joannopoulos, J. R. Wendt, G. A. Vawter, W. Zubrzycki, H. Hou, A. Alleman, Nature 2000, 407, 983. (c) Y. A. Vlasov, M. O’Boyle, H. F. Hamann, S. J. McNab, Nature 2005, 438, 65.

[10] Examples of 3D photonic crystals: (a) S.Y. Lin, J. G. Flemming, D. L. Hetherington, B. K. Smith, R. Biswas, K. M. Ho, M. M. Sigalas, W. Zubrzycki, S. R. Kurtz, J. Bur, Nature 1998, 394, 251. (b) S. Noda, K. Tomoda, N. Yamamoto, A. Chutinan, Science 2000, 289, 604.

(c) Y. A. Vlasov, X.-Z. Bo, J. C. Sturm, D. J. Norris, Nature 2001, 414, 289. (d) J. G. Flemming, S. Y. lin, I. Ei-Kady, R. Biswas, K. M. Ho, Nature 2002, 417, 52.

(e) M. Qi, E. Lidorikis, P. T. Rakich, S. G. Johnson, J. D. Joannopoulos, E. P. Ippen, H. I. Smith, Nature 2004, 429, 538.

[11] Photonic Band Gap Materials (Ed. C. M. Soukoulis), vol. 315, Kluwer Academic Publishers, Boston 1996.

[12] S. Noda, A. Chutinan, M. Imada, Nature 2000, 407, 608.

[13] (a) P. Lodahl, A. F. van Driel, I. S. Nikolaev, A. Irman, K. Overgaag, D. Vanmaekelbergh, W. L. Vos, Nature 2004, 430, 654. (b) S. Ogawa, M. Imada, S. Yoshimoto, M. Okano, S. Noda, Science 2004, 305, 227.

[14] M .Fujita, S. Takahashi, Y. Tanaka, T. Asano, S. Noda, Science 2005, 308, 1296.

[15] O. Painter, R. K. Lee, A. Scherer, A. Yariv, J. D. O’Brien, P. D. Dapkus, I. Kim, Science 1999, 284, 1819.

[16] (a) J. C. Knight, J. Broeng, T. A. Birks, P. S. J. Russell, Science 1998, 282, 1476.

(b) R. F. Cregan, B. J. Mangan, J. C. Knight, T. A. Birks, P. S. J. Russel, P. J. Roberts,

D. C. Allan, Science 1999, 285, 1537. (c) C. M. Smith, N. Venkataraman, M. T. Gallagher, D. Müller, J. A. West, N. F. Borrelli, D. C. Allan, K. W. Koch, Nature 2003, 424, 657.

[17] For recent reviews on photonic crystal fibers: (a) J. C. Knight, Nature 2003, 424, 847. (b) P. Russel, Science 2003, 299, 358.

[18] Y. Xia, J. A. Rogers, K. E. Paul, G. M. Whitesides, Chem. Rev. 1999, 99, 1823. 


\section{Unconventional strategies in nanofabrication: patterning principles}

This chapter gives a current overview of technological advancements (from 1995 to mid-2008) within the field of unconventional nanofabrication for accurate patterning in the sub-100 $\mathrm{nm}$ range. The focus lies on patterning principles that result in advancements in terms of resolution, pattern fidelity and capability to structure different materials, and on the fabrication of nanophotonic structures.

\subsection{Introduction}

1965 is the year that the co-founder of Intel $^{\circledast}$ Gordon Moore came with his prediction, now known as Moore's Law, that the number of transistors on a chip doubles every two years. ${ }^{[1]}$ Intel's commitment to Moore's Law has become a source of inspiration for several technological developments resulting in an exponential increase in the number of transistors of computer microprocessors: from 2200 in 1965 to about one billion in 2007 with the Quad-Core Intel ${ }^{\oplus}$ processor (Figure 2.1). 


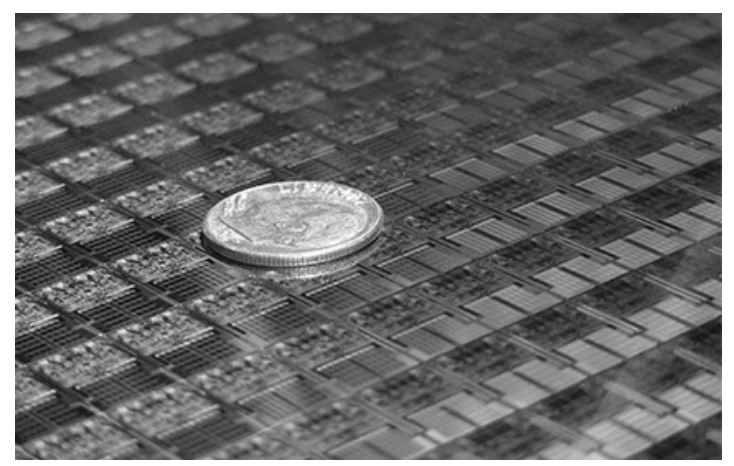

Figure 2.1 Intel's $45 \mathrm{~nm}$ high-k metal gate silicon technology is the next-generation Intel ${ }^{\circledR}$ Core $^{\mathrm{TM}}$ micro architecture. With about twice the density of the preceding $65 \mathrm{~nm}$ technology, this technology packs about double the number of transistors into the same silicon space (to about one billion for a quad-core processor). ${ }^{[2]}$

The International Technology Roadmap for Semiconductors (ITRS) ${ }^{[3]}$ is an assessment of the Semiconductor Industry Association (SIA ${ }^{[4]}$ to chart the necessary advancements in technology by identifying the technological challenges for the continuation of Moore's Law (Fig. 2.2).

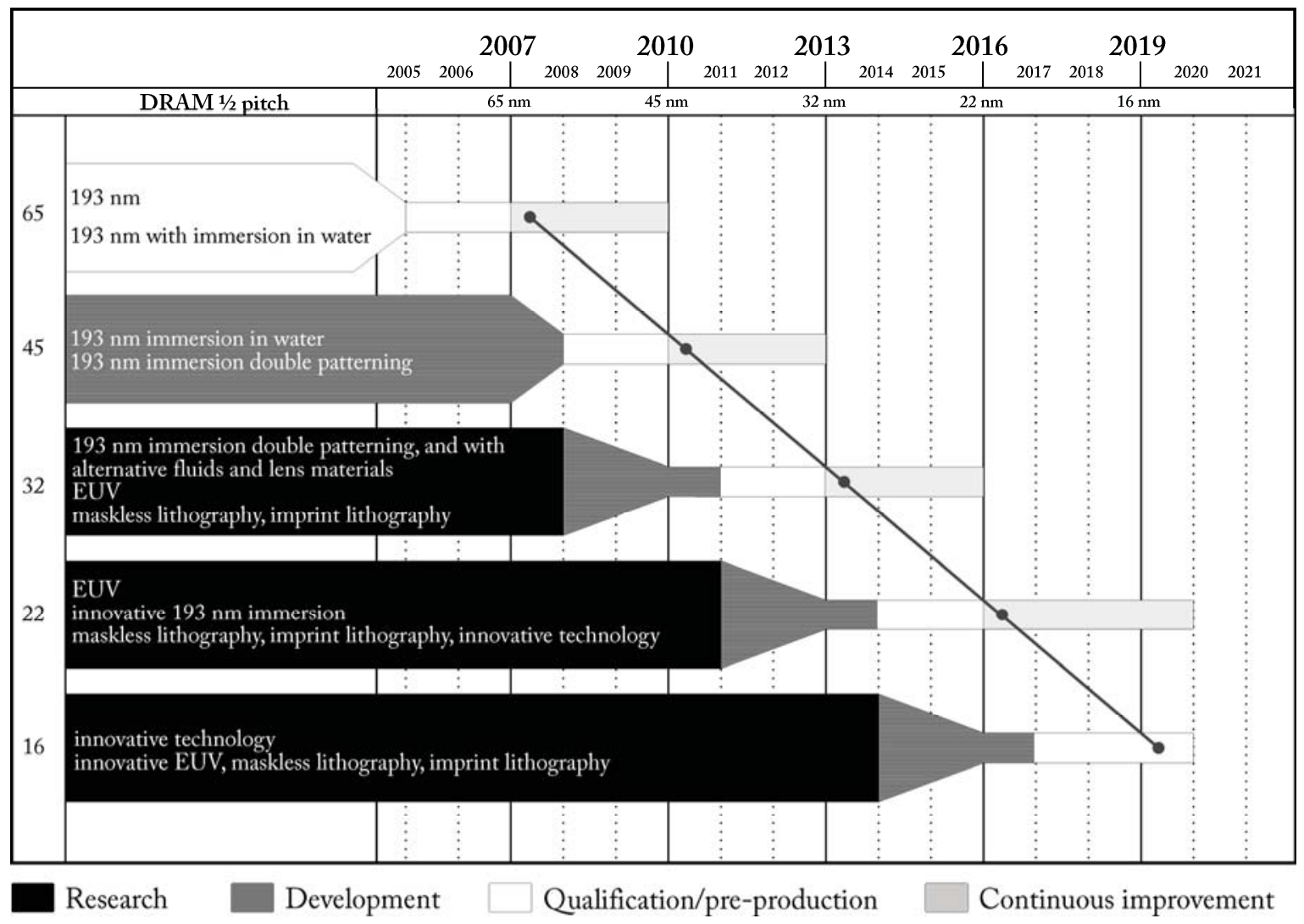

Figure 2.2 Graphical representation of the International Technology Roadmap for Semiconductors and the necessary technological advancements for maintaining the exponential decrease of the half-pitch for dynamic random-access memory (DRAM) components (dark grey line). ${ }^{[3]}$ 
The main challenge is to meet the stringent industrial requirements of cost-effective manufacturing with excellent critical dimension (CD) control in combination with accurate control of positioning (overlay) at ever-increasing resolution. ${ }^{[5]}$ As shown in Fig. 2.2, the roadmap forecasts a 45-nm node target in 2010 for deep-UV (DUV, $193 \mathrm{~nm}$ ) lithography with the ArF excimer laser, corresponding to transistor gate lengths down to $18 \mathrm{~nm}$ and a spacing of $45 \mathrm{~nm}$.

The inherent limitations of photolithography (in general) with respect to resolution (R) and depth-of-focus (DOF) are set by two scaling laws:

$$
\begin{aligned}
& \mathrm{R}=k_{1} \cdot \frac{\lambda}{N A} \\
& \mathrm{DOF}=0.5 \cdot k_{2} \cdot \frac{\lambda}{N A^{2}}
\end{aligned}
$$

where $\mathrm{R}$ is the critical dimension, $\lambda$ is the exposure wavelength, $N A$ the numerical aperture of the lens system, and $k_{1}$ and $k_{2}$ are the imaging constants. Previous strategies to extend the lifetime of optical lithography in terms of ultimate resolution with acceptable levels of DOF were aiming towards illumination sources with shorter wavelengths, optical systems with higher numerical apertures and smaller $\mathrm{k}_{1}$ values. ${ }^{[6]}$ Shorter exposure wavelengths $(<193 \mathrm{~nm})$ induce many complications to imaging optics and resist systems. The latest strategies focus on resolution enhancement techniques $^{[7]}$ such as off-axis illumination, phase-shifting masks, optical proximity correction, and immersion lithography in order to meet the key industrial requirements for cost-effective manufacturing up to the 45-nm node target. Beyond the 45-nm node, the semiconductor industry will soon run out of economically feasible strategies for DUV (193 nm) lithography thereby necessitating the research and development of alternative, next-generation lithographic technologies (NGL) such as extreme-UV (EUV) lithography, ${ }^{[8]}$ maskless lithography (ML2) ${ }^{[9,10,11,12]}$ and imprint technology ${ }^{[13]}$ (see Fig. 2.2).

NGL techniques are capable of fabricating high-resolution features; however, the development of these techniques still requires ingenuity to overcome several specific technological challenges to become feasible for cost-effective semiconductor 
manufacturing. One of the potential NGL techniques, i.e., imprint lithography, is a direct result of the technological accomplishments in the field of unconventional nanofabrication. ${ }^{[14]}$ The limitations of conventional nanofabrication in many (scientific) areas, e.g., chemistry, biology, and materials science, act as a driving force for many scientists to invent unconventional approaches to nanofabrication. These limitations include (i) low accessibility for fundamental scientific research due to high capital and operating costs, (ii) difficulty of operation, (iii) low applicability to several important classes of (bio)organic ${ }^{[15]}$ and organometallic materials, (iv) low control over surface functionality, and (v) few opportunities for innovation due to the high stage of development of conventional nanofabrication.

This chapter attempts to present a (procedural) review of unconventional nanofabrication for low-cost and fast prototyping of nanostructures, instead of dealing with extensions of conventional nanofabrication. The purpose is to illustrate only the most promising patterning principles in unconventional nanofabrication in terms of resolution, pattern fidelity (reproducibility, uniformity), and the capability to structure different materials. Conceptually, the generation and replication of patterns are two different approaches in terms of process characteristics and requirements and in fact accentuate different aspects of nanofabrication. This chapter reviews the current stateof-the-art of unconventional nanofabrication by categorizing the techniques into one of the two major tracks: generation of patterns (Section 2.2) and replication of patterns (Section 2.3). Examples of the fabrication of periodic and functional nanostructures are given in these two sections, with the emphasis to a very active and fascinating field of application: nanophotonics.

\subsection{Generation of patterns}

The conventional approach of generating high-resolution patterns is to use directwrite technologies that usually exploit a beam of photons or high energy particles to pattern matter in a subtractive and serial manner. Examples of these techniques include ion-beam lithography (IBL) ${ }^{[9]}$ electron-beam lithography (EBL) ${ }^{[10]}$ protonbeam writing, ${ }^{[16]}$ and multiphoton lithography (MPL). ${ }^{[17]}$ These techniques comply with the stringent requirements for implementation in semiconductor manufacturing 
and are applied in the fabrication of high-resolution masks. The unconventional techniques covered in this section are still in an experimental stage of development.

\subsubsection{Pattern formation by size reduction}

At present, there exists a large set of potential techniques for size reduction in case of generating periodic high-resolution features.

\subsubsection{Edge lithography}

The concept of edge lithography is to exploit the topographical edges of micrometer-scale features in order to fabricate nanometer-scale features using a costeffective patterning strategy. A summation of the edge lithographic techniques available is given in Table 2.1 .

Table 2.1 List of potential strategies in edge lithography

\begin{tabular}{|c|c|c|}
\hline Technique & Patterning principle & Resolution* \\
\hline $\begin{array}{l}\text { near-field phase-shifting } \\
\text { photolithography }\end{array}$ & $\begin{array}{l}\text { constructive and destructive interference of light using } \\
\text { transparent elastomeric masks as the optical element }\end{array}$ & $30 \mathrm{~nm}$ \\
\hline $\begin{array}{l}\text { undercutting by isotropic } \\
\text { wet-chemical etching }\end{array}$ & $\begin{array}{l}\text { lift-off to reproduce the outlines of the original template by } \\
\text { the undercut }\end{array}$ & $50 \mathrm{~nm}$ \\
\hline nanoscale spacer lithography & sidewall deposition on a sacrificial template & $<10 \mathrm{~nm}$ \\
\hline capillary force lithography & $\begin{array}{l}\text { capillary rise of polymer melts by wetting on elastomeric } \\
\text { stamps and successive break down of the meniscus at } \\
\text { temperatures above } \mathrm{Tg}\end{array}$ & $<100 \mathrm{~nm}$ \\
\hline edge-spreading lithography & $\begin{array}{l}\text { diffusion of substrate-compatible ink by guidance of a } \\
\text { topographical template }\end{array}$ & $30 \mathrm{~nm}$ \\
\hline $\begin{array}{l}\text { patterning local disorder in } \\
\text { monolayer resists }\end{array}$ & $\begin{array}{l}\text { introduction of defects by formation of SAMs at the } \\
\text { transitions of a topographical template }\end{array}$ & $50 \mathrm{~nm}$ \\
\hline
\end{tabular}


The first edge-lithographic technique, near-field phase-shifting photolithography, ${ }^{[18]}$ shares its patterning principle with contact-mode photolithography. In contrast to projection photolithography, contact-mode set ups use a transparent mask instead of projection optics. The concept is to minimize the mask-resist gap, allowing the resists to interact with the evanescent waves, i.e., nonpropagating electromagnetic modes, that modulate the intensity of the illuminating light in a region close to the mask at much higher spatial frequencies than is feasible by far-field diffraction.

The corresponding phase shift generates small regions of constructive and destructive interference of the light with lateral dimensions on the order of one-fourth of $\lambda$ (or less), reaching its optimum at mask-resist gaps that are equal to the ratio of $\lambda$ to twice the change in refractive index $(\Delta \mathrm{n})$ between air and the optical mask. ${ }^{[18 b]}$ Nearfield phase-shifting photolithography exploits this interference by projecting only the edges of the transparent mask onto the surface of the resist (Fig. 2.3a). Additionally, pattern inversion of the resulting pattern (i.e., trenches in positive-tone resist) is within the capabilities of this technique simply by changing to negative-tone resists (Fig. 2.3a).

(a)

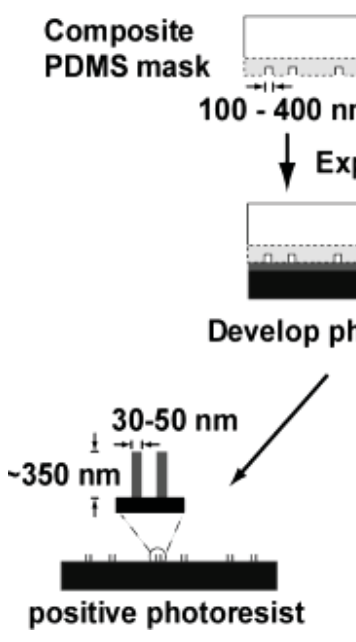

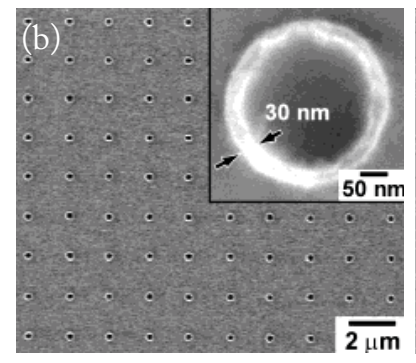
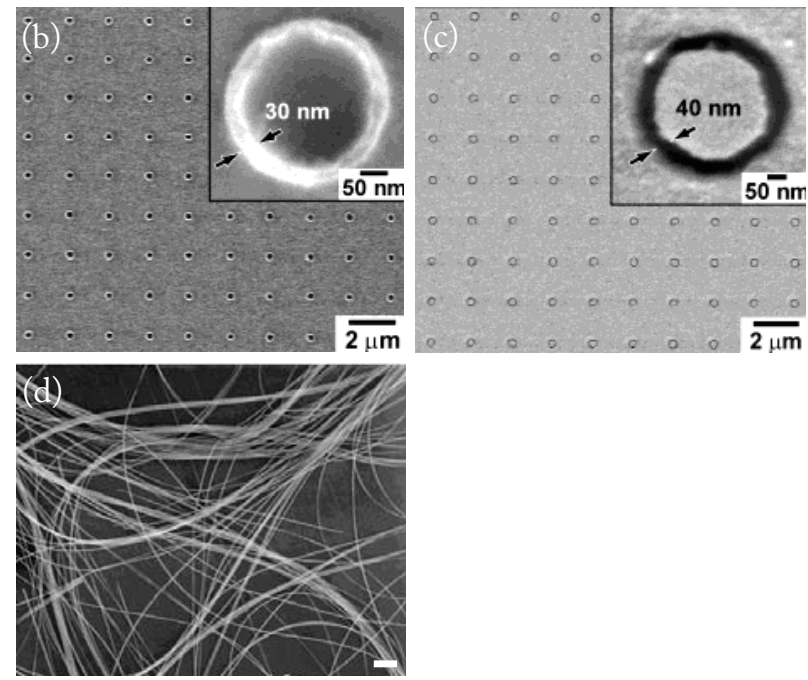

Figure 2.3 (a) Schematic representation of near-field phase-shifting photolithography for positive-tone and negative-tone resists. ${ }^{[18 c]}$ (b) Scanning electron microscope (SEM) image of $30 \mathrm{~nm}$-wide rings in positive-tone resist. $^{[18 c]}$ (c) SEM image of $40 \mathrm{~nm}$-wide trenches in palladium after wet-chemical etching using the resist as the etch mask. ${ }^{[18 c]}$ (d) SEM image of $\sim 2 \mathrm{~cm}$ long and $40 \mathrm{~nm}$-wide silicon nanowires after lift-off from the SOI substrate (scale bar: $2 \mu \mathrm{m}){ }^{[19]}$ 
The disadvantages of conventional contact-mode photo-lithography of using elaborate vacuum systems to bring hard masks in close contact with the resist over large areas potential damage and contamination of the mask - are overcome by incorporation of elastomeric poly(dimethylsiloxane) (PDMS) masks. These masks are also optically transparent, facilitate conformal contact over large areas without application of any external force and, by this means, take advantage of the full potential of the near-field optics. Fig. 2.3b illustrates this fact by the fabrication of features in positive-tone resists as small as $\sim 30 \mathrm{~nm}$.

As this technique is only applicable to photosensitive materials, Whitesides et al. have taken the resulting resist patterns to the next level by patterning different metals on surfaces using lift-off ${ }^{[18 a]}$ and selective wet-chemical etching (Fig. 2.3c) and by generating $80 \mathrm{~nm}$-wide single-crystalline silicon nanowires with lengths over $2 \mathrm{~cm}$ (Fig. 2.3d). Several potential applications in optics of the resulting metal nanowires on surfaces include nanowire polarizers, ${ }^{[18 a]}$ optical notch filters, optical memories, and diffraction gratings with subwavelength features. By addition of amplitude modulating components in the form of thin metal layers onto the elastomeric mask, Schmid et al. have found a means to attain more flexibility in the generation of different types of patterns in resists. ${ }^{[20]}$ Additional resolution improvements are foreseen by reducing the thickness of the resist, by increasing the index of refraction of the resist and by using surface-sensitive resists.

The second edge-lithographic technique also exploits a well-known strategy in semiconductor manufacturing - undercutting (overetching) by isotropic wet-chemical etching. ${ }^{[21]}$ The concept is to use one resist mask to perform two sequential processes: (1) overetching to create a small undercut, and (2) metal lift-off to reproduce the edges of the resist pattern. The procedure of undercutting metal films by isotropic wetchemical etching ${ }^{[22]}$ as a means to nanostructure films over large areas $\left(>4 \mathrm{~cm}^{2}\right)$ is shown in Fig. 2.4a. Wet-chemical etching of polycrystalline metal films is isotropic by nature, resulting in convex sidewalls. For this reason, the minimum undercut depends on the film thickness and the grain size of the metal. For chromium films (with grain sizes of $~ 15-25 \mathrm{~nm}$ ), the minimum feature size increases from $50 \mathrm{~nm}$ (for a film thickness of $10 \mathrm{~nm}$, Fig. 2.4b) to $100 \mathrm{~nm}$ (for a film thickness of $35 \mathrm{~nm}$ ). ${ }^{[22]}$ Additionally, AFM studies have shown that most etch systems are sensitive to grain 
boundaries of polycrystalline metals, ${ }^{[23]}$ therefore suggesting that the grain size is a limiting factor to high-resolution patterning with respect to the line-edge roughness (LER) of the final features. Concerning the impact of the material aspects in undercutting, higher resolution and LER are within reach in case of thin films of amorphous materials (e.g., alloys, glass, etc.) using this technique. A technical advantage of undercutting is the lateral dimension control by the etch time. Strong bandpass filters represent one application of the resulting patterns in optics, as shown in Fig. 2.4c in the case of two-dimensional arrays of slots in chromium on optical grade $\mathrm{Si} / \mathrm{SiO}_{2}$.

(a)
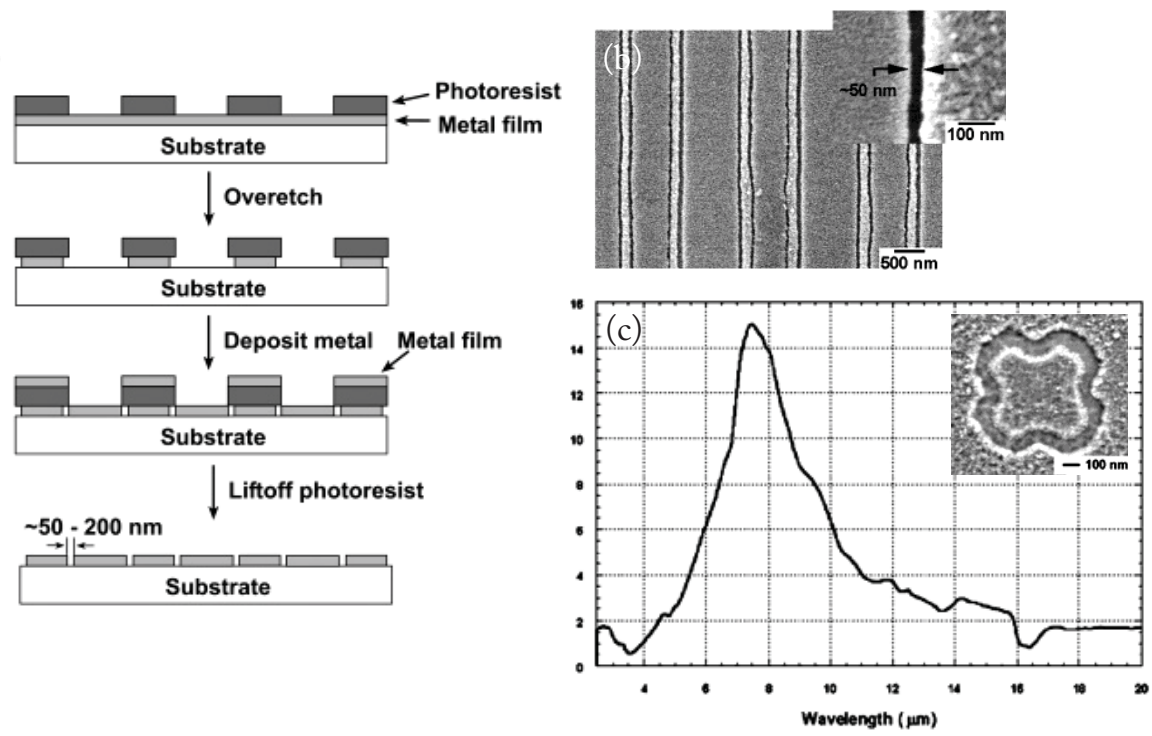

Figure 2.4 (a) Schematic representation of undercutting by isotropic wet-chemical etching. (b) SEM image of $50 \mathrm{~nm}$-wide trenches in chromium and (c) infrared (IR) transmission spectrum of an array of loops in aluminum (see inset) on a substrate of optical grade $\mathrm{Si} / \mathrm{SiO}_{2} \cdot{ }^{[22]}$

The next edge-lithographic technique originates from the area of microelectromechanical systems (MEMS) in the fabrication of gate sidewall spacers: spacer lithography. Several forms exist in edge lithography, ${ }^{[24,25,26]}$ with one common concept: conformal deposition of a thin film of a material over a topographical template of a sacrificial material in combination with successive etching of the sacrificial support to release the final nanostructures (Fig. 2.5a).

The main advantages of this technique are its ultimate resolution with accurate control over the lateral feature dimensions by means of the film thickness during conformal deposition. Additionally, this technique enables iterative processing by 
alternating the two materials in order to generate high pattern densities by multiplication. ${ }^{[24 a, 25]}$ This is an exclusive feature for edge lithographic techniques and overcomes the main disadvantage of low pattern density. Sub-10 nm resolution is within the capabilities, however, at the expense of materials choice for patterning. Potential applications of the resulting structures include the use as hard molds for nanoimprint lithography (see Section 2.3.2) ${ }^{[24,26]}$ and as nanochannels (Fig. 2.5b). Recently, a gas-sensing device for $\mathrm{H}_{2}$ and $\mathrm{CO}$ was reported using this technique for the fabrication of the responsive 70 - $\mathrm{nm}$ wide $\mathrm{ZnO}$ nanowires (Fig. 2.5c).

(a)
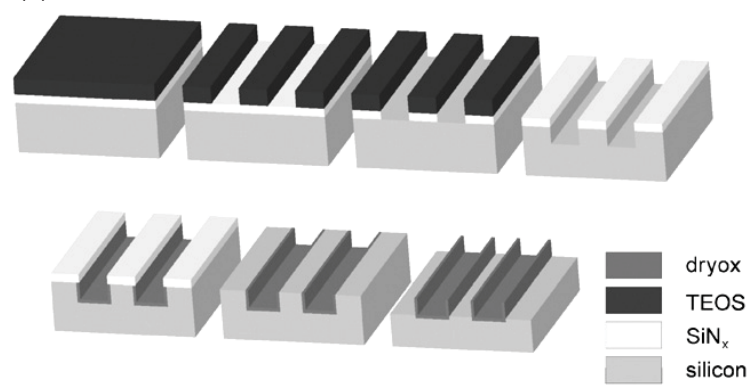

(b)

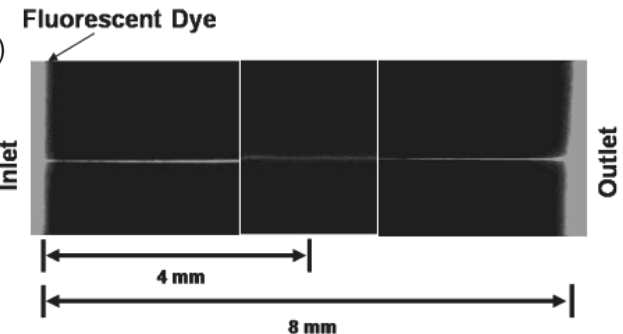

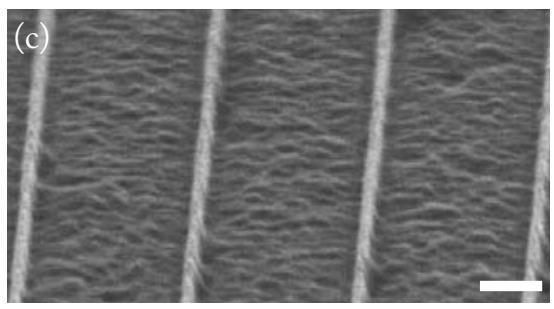

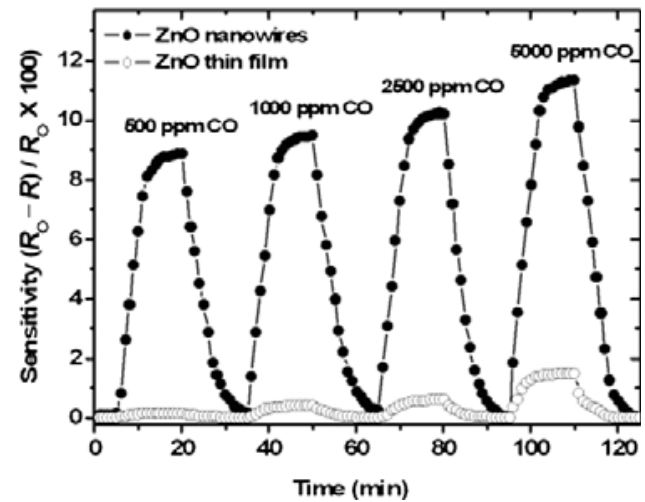

Figure 2.5 (a) Schematic representation of spacer lithography. ${ }^{[26]}$ (b) Fluorescent microscopy images of a continuous flow of water containing a fluorescent dye at three positions along a $1.5 \mathrm{~cm}$-long $30 \mathrm{~nm}$-wide nanochannel. ${ }^{[27]}$ (c) SEM image of the $\mathrm{ZnO}$ nanowires (scale bar: $250 \mathrm{~nm}$ ) and sensitivity curves of the corresponding devices and $\mathrm{ZnO}$ thin films at various concentrations of $\mathrm{CO}$ in the range of 500-5000 ppm at $200{ }^{\circ} \mathrm{C} \cdot[28]$

Capillary force lithography $(\mathrm{CFL})^{[29]}$ is a technique that combines the essential feature of nanoimprint lithography (NIL) ${ }^{[13]}$ - molding a polymer melt - with the key element of soft lithography ${ }^{[30]}$ - molding with elastomeric stamps for large-area patterning $\left(>16 \mathrm{~cm}^{2}\right)^{[29 b]}$ of thin polymer films (with respect to the feature height of the stamp) without the use of external force (Fig. 2.6a). The concept of this technique is the capillary rise of a polymer at temperatures above the glass-transition temperature due to a lowering of the total free energy upon wetting of the elastomeric mold. 
Several theoretical studies have been carried out on the kinetics of the resulting capillary rise, ${ }^{[31]}$ the transient formation and breakdown of the meniscus in microchannels ${ }^{[32]}$ during the patterning of thin polymer films using permeable molds. These have shown that under certain conditions (polymer film thickness, dimensions on the elastomeric stamp, and film-substrate interactions) the minimum height at the center of the meniscus can reach a minimum level at which London forces induce instability across the channel, resulting in the breakdown of the meniscus and partial exposure of the substrate (Fig. 2.6b). ${ }^{[32 a]}$ This was proven by the selective electroless deposition of copper on palladium surfaces. ${ }^{[29 a]}$

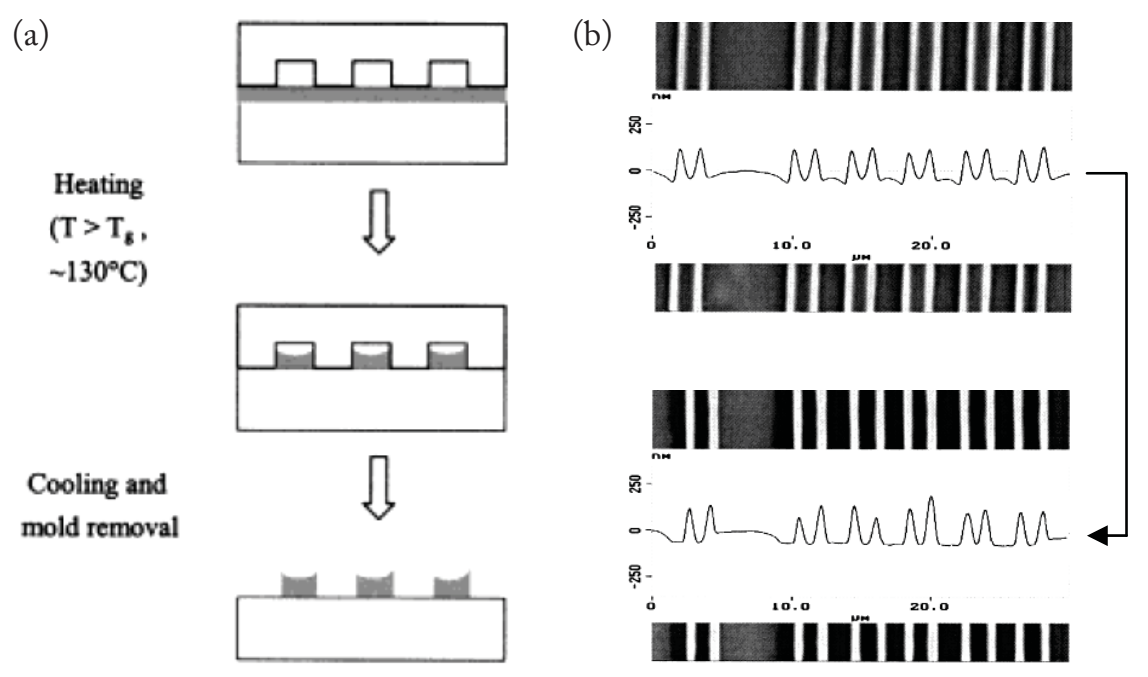

Figure 2.6 (a) Schematic representation of capillary force lithography. ${ }^{[29]}$ (b) AFM images at two stages of the process illustrating the meniscus breakdown. ${ }^{[32 a]}$

Several potential applications of these polymer templates in edge lithography can be found in Chapters 4 and 5 of this thesis, illustrating the sub-100 nm patterning capabilities of CFL. Additionally, as Sections 2.2.3 and 2.3.2 will illustrate, CFL is capable of generating also more complex patterns in polymers.

Edge-spreading lithography $(\mathrm{ESL})^{[33]}$ also originates from the combination of features from two different techniques, that is microcontact printing ${ }^{[30]}$ - printing molecular inks with elastomeric stamps - and nanosphere lithography (NSL) ${ }^{[34]}$ - self-assembly of colloidal particles on surfaces (Fig. 2.7a). These colloidal particles facilitate the diffusion of ink molecules from a planar elastomeric stamp by guidance along its surface to form a self-assembled monolayer (SAM) on the ink-compatible surfaces. 
The guidance is not restricted to colloidal particles, as was shown soon after by the use of resist patterns. As reactive spreading ${ }^{[35]}$ is responsible for the SAM formation on coinage metals, control over the lateral feature dimension depends essentially on the ink supply by means of the contact time of the planar stamp and the ink concentration. ${ }^{[33]}$ As with any technique that takes advantage of molecular diffusion for patterning surfaces, the diffusion is also a source of the loss of resolution. ${ }^{[36]} \mathrm{ESL}$ in its present form could profit from using low-diffusion inks ${ }^{[37]}$ and/or nanoparticles ${ }^{[38]}$ to increase the resolution, up to now $140 \mathrm{~nm}$ features in gold after wet-chemical etching, to the sub-100 nm range and has more potential in the generation of multiple SAMs (Fig. 2.7b) ${ }^{[39]}$ and chemical gradients (Fig. 2.7c). ${ }^{[40]}$ Additionally, the resulting molecular patterns were functional as suitable templates for site-specific adsorption of nanoparticles on surfaces. ${ }^{[41]}$ Extensions of this technique include substituting flat stamps for stamps with a topographical surface in order to generate more complex patterns. ${ }^{[33 \mathrm{~b}]}$

(a)
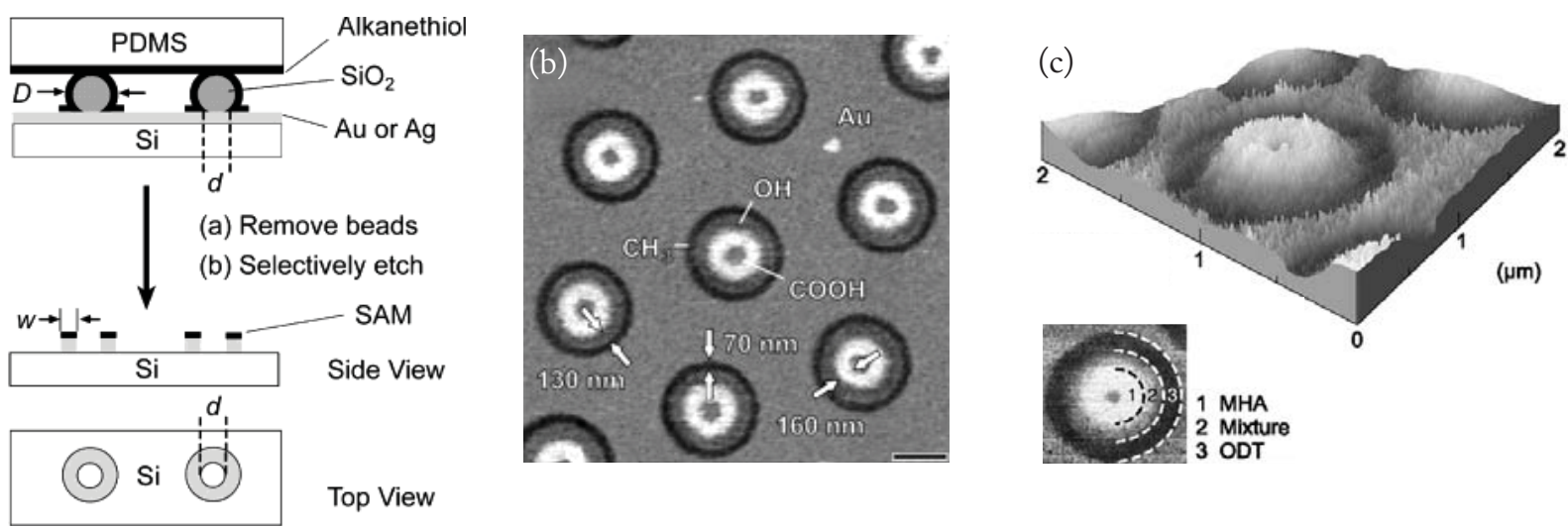

Figure 2.7 (a) Schematic representation of edge-spreading lithography. ${ }^{[33 a]}$ (b) Lateral force microscopy (LFM) image of concentric rings after consecutive printing of three inks with different functionalities (scale bar: $500 \mathrm{~nm}){ }_{.}^{[39]}$ (c) LFM image of a gradient pattern, consisting of two SAMs of different functionalities. ${ }^{[40]}$

Finally, the last strategy in this class for patterning nanostructures relies on the generation of local disorders in SAMs on topographical patterns (Fig. 2.8a). ${ }^{[42]}$ Generally, SAMs are excellent etch masks to protect surfaces against wet-chemical etchants owing to their high surface coverage, high order and low density of defects (as low as $\sim 5$ defects $/ \mathrm{mm}^{2}$ ), ${ }^{[43]}$ especially in combination with defect-tolerant and 
directional wet-etch systems. ${ }^{[44]}$ The concept of this technique is to introduce step edges in the substrate by means of lithography in order to control the positions of transition zones with lower coverage and order during SAM formation. These zones are found to provide nucleation sites for the preferential etching and deposition of materials, allowing respectively the generation of features as small as $50 \mathrm{~nm}$ (Fig. 2.8b) in a range of materials (gold, silver, $\mathrm{SiO}_{2} /$ silicon) and the crystallization of calcite crystals from a $\mathrm{CaCl}_{2}$ solution. ${ }^{[42]}$

(a)
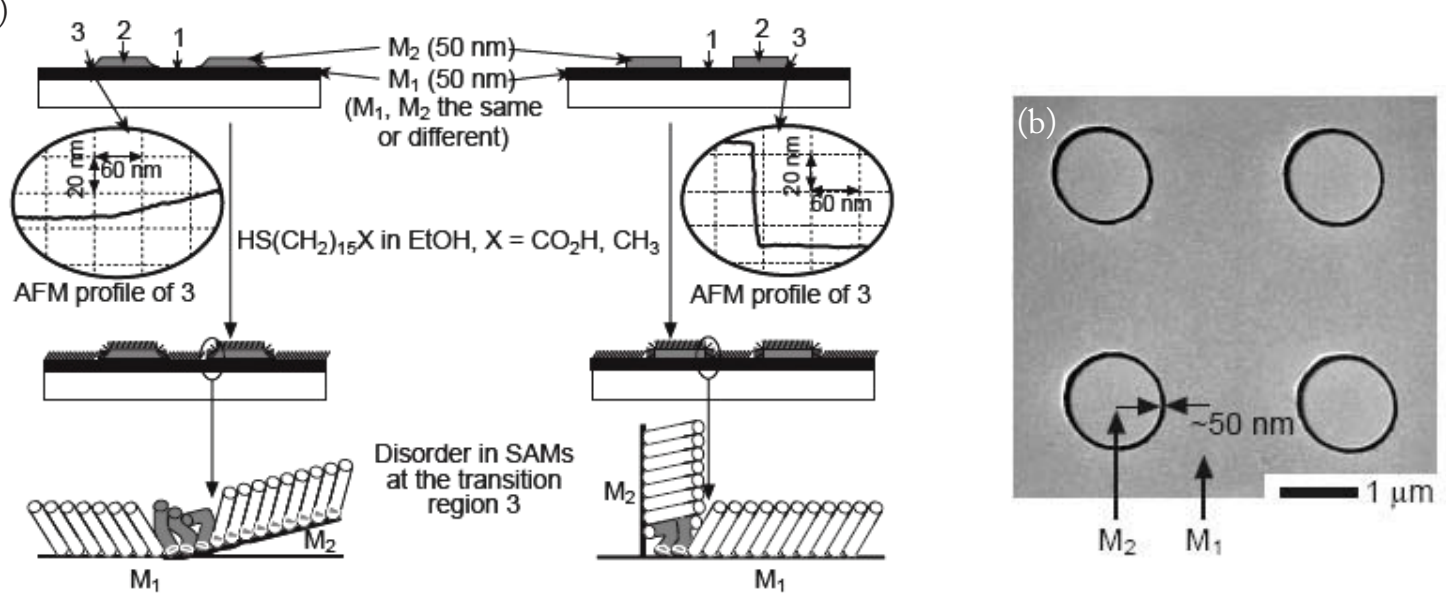

Figure 2.8 (a) Schematic representation for the generation of local disorder in alkanethiol monolayers on topographical substrates. (b) SEM image of $50 \mathrm{~nm}$ trenches by selective etching silver-on-silver substrates (i.e., $\left.\mathrm{M}_{1}=\mathrm{M}_{2}=\mathrm{Ag}\right){ }^{[42 \mathrm{a}]}$

Additionally, these zones are also more susceptible to exchange with molecules in solution, resulting in pattern reversal during wet-chemical etching. The incorporation of a thin layer of titanium in between the two metal layers is another strategy to generate a transition zone to facilitate the preferential electrodeposition of copper into nanowires with lateral dimensions as small as $70 \mathrm{~nm} .{ }^{[45]}$ 


\subsubsection{Additional strategies to size reduction}

A number of techniques are capable of sub-100 $\mathrm{nm}$ patterning by application of size reduction strategies. A summation of the size reduction techniques available is given in Table 2.2.

Table 2.2 List of additional strategies to size reduction

\begin{tabular}{|c|c|c|}
\hline Technique & Patterning principle & Resolution* \\
\hline maskless photolithography & $\begin{array}{l}\text { constructive and destructive interference of light using the } \\
\text { resist pattern as the optical element }\end{array}$ & $50 \mathrm{~nm}$ \\
\hline lithographic molding & anisotropic etching of $<100>$ silicon & $40 \mathrm{~nm}$ \\
\hline replica molding & $\begin{array}{l}\text { molding against elastomeric stamps under isotropic } \\
\text { deformation (compression, stretching, bending or a } \\
\text { combination of these deformations) }\end{array}$ & $30 \mathrm{~nm}$ \\
\hline sectioning with a microtome & $\begin{array}{l}\text { sectioning thin metal films in an encapsulating matrix of a } \\
\text { polymer }\end{array}$ & $20 \mathrm{~nm}$ \\
\hline $\begin{array}{l}\text { superlattice nanowire pattern } \\
\text { transfer }\end{array}$ & $\begin{array}{l}\text { transfer of metal nanowires using a superlattice as a } \\
\text { supporting template }\end{array}$ & $8 \mathrm{~nm}$ \\
\hline nanopantography & $\begin{array}{l}\text { focusing of a broad-area ion beam using arrays of circular } \\
\text { features in a metal/insulator layer as microlenses }\end{array}$ & $10 \mathrm{~nm}$ \\
\hline
\end{tabular}

* The resolution values in this table represent the smallest feature dimensions that have been shown to date with the respective technique, but do (in most cases) not represent the ultimate limit.

The first in line is maskless photolithography, ${ }^{[46]}$ a technique that exploits the direct interaction of resist patterns upon flood-illumination with incoherent and polychromatic light. This technique is the maskless form of near-field phase-shifting photolithography (Section 2.2.1.1). As a substitute, the resist patterns serve as optical elements to phase-shift and direct the incident light into the resist during exposure (Fig. 2.9a). As the resist pattern is the optical element, the final patterns after resist development strictly depend on the contours of the initial resist patterns. ${ }^{[46]}$ The same resolution improvements for near-field phase-shifting photolithography also apply for this technique. 
The procedure for lithographic molding ${ }^{[47]}$ starts with the fabrication of a grating by anisotropic etching of $\langle 100\rangle$ silicon in an aqueous $\mathrm{KOH}$ solution. As the procedure relies on the anisotropic etching along the $<111>$ crystal plane of the silicon, the resulting template combines an ultra-smooth surface with excellent feature dimension control over large areas. The triangular profile with its sharp radius of curvature at the tip of the profile $(\sim 40 \mathrm{~nm})^{[47]}$ is practical in the molding of elastomeric stamps for printing ${ }^{[47,48]}$ and for the application as hard molds in nanoimprint lithography. ${ }^{[49]}$ Fig. $2.9 \mathrm{~b}$ is illustrative for the feasibility of nanocontact printing using the stamps to print high-resolution molecular patterns on surfaces with high reproducibility, in this case of a fourth generation poly(amidoamine) dendrimer (G4-PAMAM) on silicon. The accuracy of printing with high-molecular-weight inks at these dimensions demonstrates that only the sharp tips of the composite stamp come into contact with the surface.
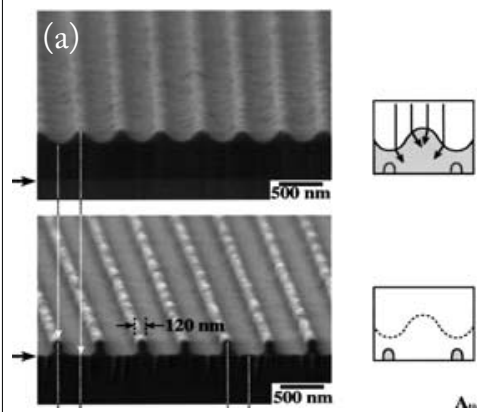
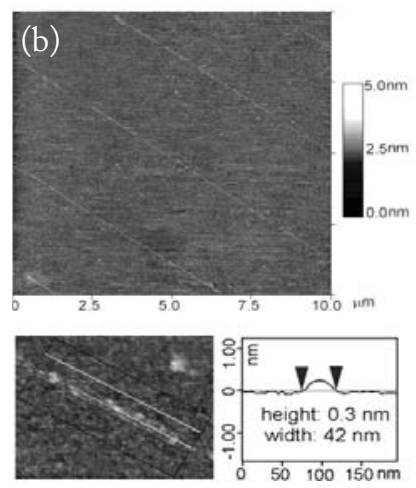

(c)
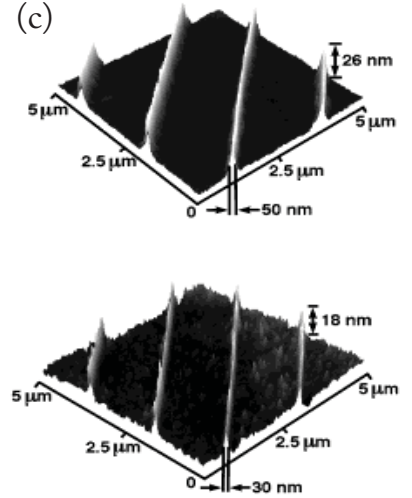

Figure 2.9 (a) SEM images of the resist pattern before and after flood-exposure and development in maskless photolithography, with the drawings illustrating the interaction of the resist with the light. ${ }^{[46]}$ (b) AFM images of $42 \mathrm{~nm}$-wide dendrimer lines on a silicon surface after microcontact printing, including a high-resolution image and average cross-section. ${ }^{[48]}$ (c) Series of AFM images of the master structure (top) and replica in PU (bottom) after one cycle of deformation by bending. ${ }^{[50 \mathrm{~b}]}$

Replica molding is the basis of any soft-lithographic technique and comprises the solidifying of a liquid precursor against hard molds in high accuracy over large areas (see Section 2.3.2). The elasticity of PDMS facilitates the manipulation of the feature dimensions by mechanical deformation of the stamp (compression, stretching, bending or a combination of these deformations). The concept is to perform several cycles consisting of two basic molding steps: (1) replica molding of UV-curable polyurethane (PU) against PDMS stamps under deformation, (2) replica molding of PDMS against 
the PU replica. ${ }^{[50]}$ The ability to make positive as well as negative polymeric replicas and to reduce the lateral feature dimensions adds a lot of flexibility to this potential technique. Fig. 2.9c illustrates the effect on the lateral feature dimensions after one cycle of replica molding under mechanical deformation, resulting in lateral feature dimensions as small as $30 \mathrm{~nm} \cdot{ }^{[50 \mathrm{~b}]}$ Additionally, the isotropic deformation of the PDMS mold also permits the fabrication of structures with gradients in lateral feature dimensions and of complex structures, such as diffraction gratings on planar and bent surfaces and features on hemispherical objects. The principal challenge with exploiting uniform deformations is to attain a high level of uniformity at the sub-100 nm scale.

The next two techniques take advantage of deposition techniques that are capable of growing thin films over large areas by translating the resulting films into structures with lateral dimensions corresponding to the thickness of the film (see Fig. 2.10). One strategy to expose the cross-section of a metal film is to incorporate the thin film into a soft encapsulating matrix and subsequent sectioning the matrix with a microtome (Fig. 2.10a). ${ }^{[51]}$ The last step is the most challenging aspect of this technique in order to minimize the delamination at the matrix-metal interface. The second strategy is more generally applicable and results in ultrahigh-density arrays of metal and/or semiconductor nanowires on any surface. The technique, superlattice nanowire pattern transfer (SNAP), ${ }^{[52]}$ owes its name from exploiting a superlattice of alternating materials grown by molecular beam epitaxy (MBE) in order to transfer metal nanowires onto the surface of a substrate (Fig. 2.10b). The technological advantage is the direct control over the spacing and the lateral dimensions of the resulting nanowires by the thicknesses of the alternating layers. Successful implementation of these arrays in nanofabrication has been shown in the fabrication of nanoimprint molds. ${ }^{[53 a]}$ The highest density to date consists of an array of 20 platinum nanowires, $8 \mathrm{~nm}$ in diameter with a spacing of $16 \mathrm{~nm}$. The nanowires have high aspect ratios (up to $10^{6}$ ) and, in addition, it is possible to perform the transfer process multiple times to generate simple circuits with nanowire junction densities of over $10^{11}$ junctions $\cdot \mathrm{cm}^{2}$. Promising nanomechanical devices incorporating such high-density arrays of nanowires include high-frequency resonators ${ }^{[52]}$ and high-performance nanowire fieldeffect transistors (FETs). ${ }^{[53 c]}$ 
(a)
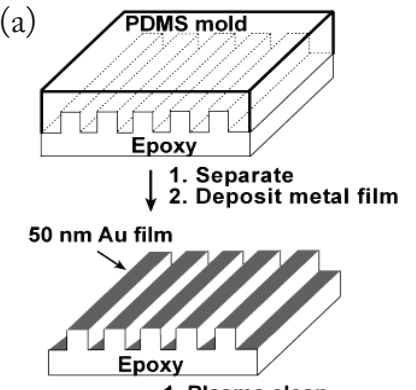

1. Plasma clean 2. Embed in epoxy
3.Thermally cure epoxy

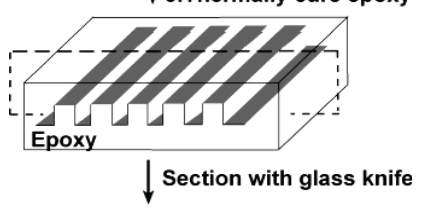

Section with glass knife
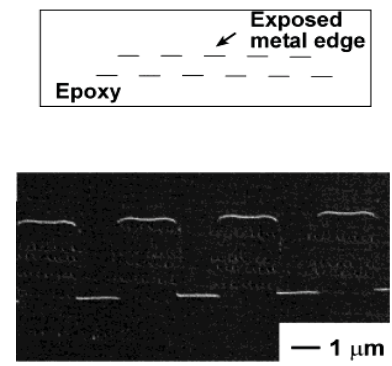
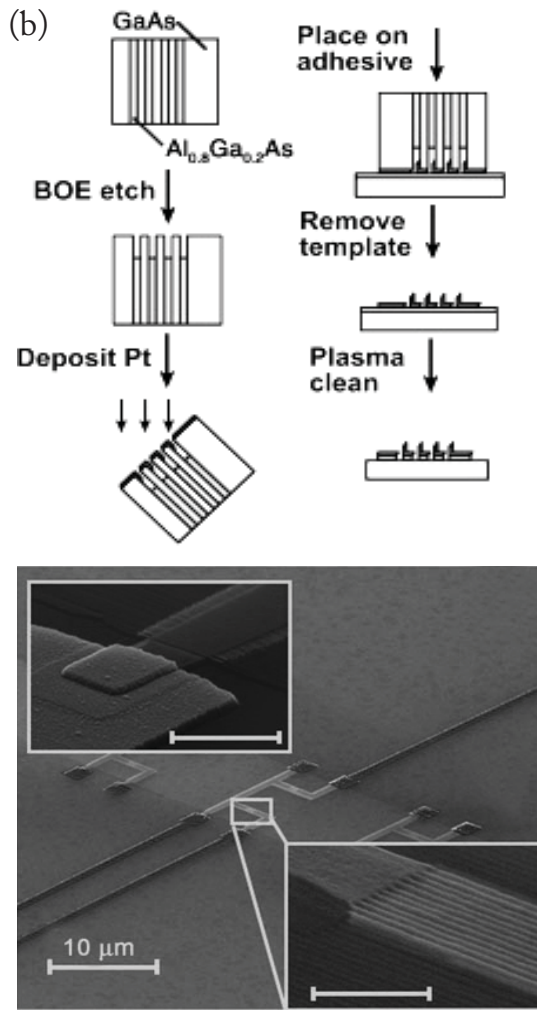

Figure 2.10 (a) Schematic representation of sectioning thin films with a microtome and a SEM image of $50 \mathrm{~nm}$-wide gold features in a continuous epoxy matrix. ${ }^{[51]}$ (b) Schematic representation of the SNAP technique ${ }^{[52]}$ and a SEM image of an inverter with silicon nanowire source-drain regions in connection with large silicon bars (bottom inset) that contain the metal -wire contacts (top inset) (scale bars: $500 \mathrm{~nm}$ ). ${ }^{[53 c]}$

The most recent technique in this section (nanopantography) exploits arrays of circular features in a metal/insulator multilayer as ion-focussing microlenses to write nanopatterns in parallel into the underlying substrate by applying appropriate voltages to the lens electrodes during exposure of a broad-area ion beam. ${ }^{[54]}$ Using this technique, the ion beam focuses in spots that are about 100 times smaller than the lateral dimension of the microlenses. ${ }^{[54]}$ As the lens array is part of the substrate, nanopantography is not susceptible to misalignment errors due to vibrations or thermal expansion. Strategies to generate a range of patterns include continuous tilting the substrate during exposure to the beam and addressing individual microlenses by patterning of the top metal layer for isolation. ${ }^{[54 b]}$ 
patterning principles

\subsubsection{Pattern formation by writing}

Writing is the main process in the semiconductor industry for generating arbitrary patterns on surfaces and in most cases involves serial techniques that require large capital investments. Examples of serial techniques include ion-beam and electronbeam lithography. ${ }^{[9,10]}$ Unconventional techniques, also denoted by the general term of scanning-probe lithography (SPL), ${ }^{[12]}$ employ a rigid stylus for generating highresolution structures by means of material modification, deposition, and removal of material in a mechanical, physical, optical, electrical, or chemical fashion. SPL in general has several strengths, including nanoscale spatial resolution (on the order of $10 \mathrm{~nm}$ ), excellent reproducibility, low-cost facilities, basic operating procedures and the capability of real-time imaging of the patterns for accurate alignment at the nanometer level. ${ }^{[12 b]}$ For most of the SPL techniques, however, the main challenge remains the intrinsic low writing speed. Among the best known examples of parallel writing operations is the Millipede system of IBM to read-and-write in thin polymer layers for data-storage. ${ }^{[55]}$

This section will cover dip-pen nanolithography $(\mathrm{DPN})^{[56]}$ as the most prevalent technique for add-on writing by means of probes in parallel over the surface to deliver chemical reagents in nanoscopic regions on the substrate (Fig. 2.11).

(a)

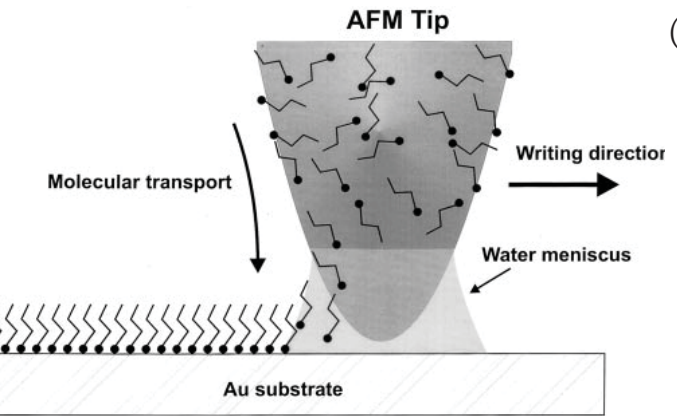

(b)

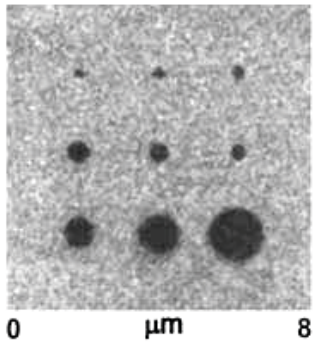

Figure 2.11 (a) Schematic representation of the DPN process of writing structures of molecules by means of scanning an ink-containing atomic force microscopy (AFM) probe across the surface. The water meniscus is responsible for the molecular transport to surfaces. ${ }^{[56]}$ (b) LFM image and scaling analysis for patterning dots (by holding the probe in stationary contact with the surface) of octadecanethiol (ODT) on gold. ${ }^{[63 b]}$

DPN is possibly the only lithographic technique that offers high-resolution and registration with direct-writing capabilities and is accessible to anyone that can use the 
AFM. This is the reason for the fast development of DPN after its introduction in $1999,{ }^{[56 a]}$ as shown by the number of contributions within the first 3 years. ${ }^{[57]}$ During this time, DPN was found to be compatible with small organic molecules, ${ }^{[56,58]}$ organic polymers, ${ }^{[59]}$ biological molecules $\left(\mathrm{DNA}^{[60 a]}\right.$ and collagen ${ }^{[60 b]}$ ), colloidal particles ${ }^{[61]}$ and metal ions ${ }^{[62]}$ for patterning surfaces ranging from metals to insulators and on top of functional monolayers. Of high importance for future biological device applications (e.g., detection in biomolecular assays) and studies on hierarchical assembly processes of biological systems was the experimental proof that writing by DPN preserves the structure, functionality and activity of biomolecules on surfaces. ${ }^{[60 b]}$ The probesubstrate ink transport process is a complex process influenced by a range of parameters, including temperature, ambient humidity, water solubility of the ink, condition and chemical composition of the surface and the ink, distribution and mobility of the ink on the probe, and the shape of the probe. Understanding of these parameters is important and would facilitate efforts for developing new ink-substrate combinations and inking systems, for improvement of the ultimate resolution, and for the fabrication of custom probes. Several systematic studies illustrate that the dependence of the deposition rates on temperature and ambient humidity originate from the water solubility of the ink. ${ }^{[63,64]}$ Additionally, the correlation of time with deposition rate, as shown in Fig. 2.11b for ODT on gold, is a characteristic feature applicable to a large range of different molecules (only with different deposition rate constants) and is consistent with the probe serving as a source of constant ink flux. ${ }^{[65]}$ As is the case for SAMs made from solution, the resulting monolayers by DPN are of high quality and can function as excellent etch resists in the etching of different metals and semiconductors. ${ }^{[66]}$

DPN offers several exclusive features of industrial importance in terms of site selective exchange ${ }^{[58 b]}$ to fabricate complex nanostructures and also erasure to allow elimination or repair of defective nanostructures (Fig. 2.12). ${ }^{[67]}$ DPN also allows the direct patterning of metal nanostructures by electrochemical DPN (E-DPN), ${ }^{[62 a]}$ in which a conductive probe serves as the electrode with the water meniscus as a nanoscale electrochemical cell to deposit metals $(\mathrm{Pt}, \mathrm{Au}, \mathrm{Ag}, \mathrm{Cu}, \mathrm{Pd})$ by means of electrochemical reduction of metal ions. 
patterning principles
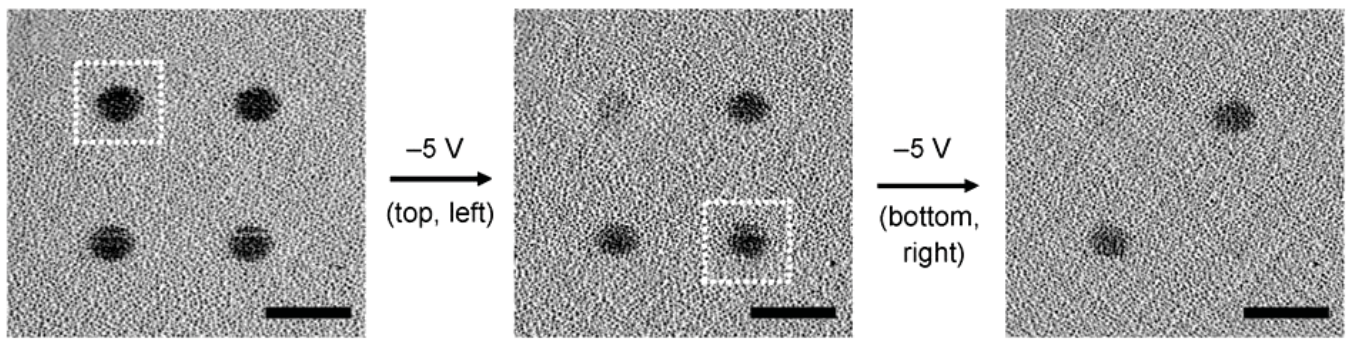

Figure 2.12 LFM images showing the selective elimination of 16-mercaptohexadecanoic acid (MHA) dots by conductive AFM. The initial array consists of $500 \mathrm{~nm}$ in diameter dots of MHA in a background of ODT. Successive selective electrochemical elimination of MHA in two areas on the surface is shown by scanning a conductive probe at a potential of $-5 \mathrm{~V}$ containing ODT over the specific areas on the surface. ${ }^{[67]}$

Scientific research has put a lot of effort into shifting the paradigm of DPN in terms of the low intrinsic writing speeds of a few micrometers per second by highthroughput solutions. Starting from the implementation of passive probe arrays of 8, ${ }^{[56 b]} 26$ (Fig. $2.13 \mathrm{a}$ ), ${ }^{[68 \mathrm{a}]}$ and 55000 probes (Fig. 2.13c) ${ }^{[69]}$ by micromachining technology, ${ }^{[70,71 a]}$ new types of elastomeric probes ${ }^{[71]}$ to active parallel-probe arrays by thermomechanical probe actuation and microfluidic ink delivery systems, ${ }^{[72]}$ the scientific community has come with a lot of interesting solutions toward highthroughput writing. NanoInk ${ }^{[73]}$ is a company that takes DPN writing to the commercial level by providing several potential production tools (see Fig. 2.13).
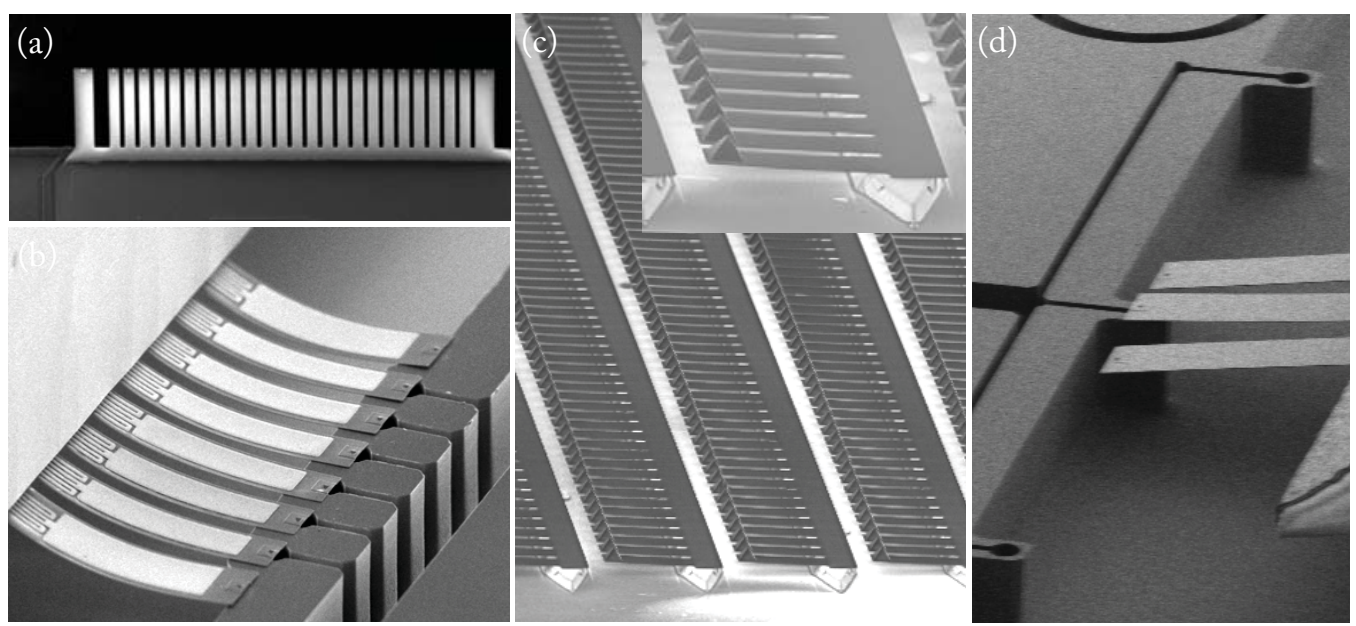

Figure 2.13 SEM images of different technological advancements in DPN. ${ }^{[73]}$ (a) Passive multi-probe array, consisting of 24 identical writing probes with two flanking probes of the same length - the left one is the reader probe, the right one is allows for better leveling of the array. (b) ActivePen ${ }^{\mathrm{TM}}$ option for addressing individual probes in one multi-probe array, include features as multilayer writing with separate probes and selective parallel writing. (c) 2D nano PrintArray ${ }^{\mathrm{TM}}$ consisting of 55000 probes across $1 \mathrm{~cm}^{2}$. (d) Inkwell ${ }^{\mathrm{TM}}$ arrays for selective inking of the probes. 
Polymer pen lithography $(\mathrm{PPL})^{[74]}$ is a very recent technique that merges feature size control and registration capabilities of DPN with large-area capability of contact printing by using soft elastomeric probe arrays (up to 11 million probes) and allows lateral feature dimensions ranging from $90 \mathrm{~nm}$ to hundreds of micrometers by changing the force and contact time.

\subsubsection{Pattern formation by self-assembly}

Self-assembly is the spontaneous organization of two (or more) constituents into larger structures in two or three dimensions by noncovalent forces and is ubiquitous in nature. ${ }^{[75]}$ The concept of self-assembly is that the final structure is close to or at thermodynamic equilibrium owing to the dynamic and reversible interactions of the self-assembling constituents. The characteristics of these constituents predetermine the final structure, therefore control over the self-assembly process into the final structure is within reach by tailoring the properties of the constituents in terms of surface topography and functionality. From a technological perspective, self-assembly is low-cost, fast, self-healing for irregularities and scalable, and several of the selfassembling systems have large potential in nanofabrication.

\subsubsection{Block copolymer lithography}

Block copolymers (BCPs) are a special class of polymers with two (or more) polymer chains bound together through one covalent bond. ${ }^{[76]}$ Generally, most combinations of polymers are immiscible for entropic reasons and have a strong tendency to phase separate. ${ }^{[77]}$ Owing to the constraints by the covalent bond, the different polymer chains in BCPs can only intermolecularly phase separate to a distance compatible with the size of the polymer chains (i.e., $\sim 10-100 \mathrm{~nm})^{[78]}$ resulting in the self-assembly of a variety of high-resolution periodic domains at equilibrium. For coil-coil diblock copolymers, the molecular weight, volume fraction of the components and the degree of polymer incompatibility determine the domain structure in the bulk state (Fig. 2.14). 
patterning principles

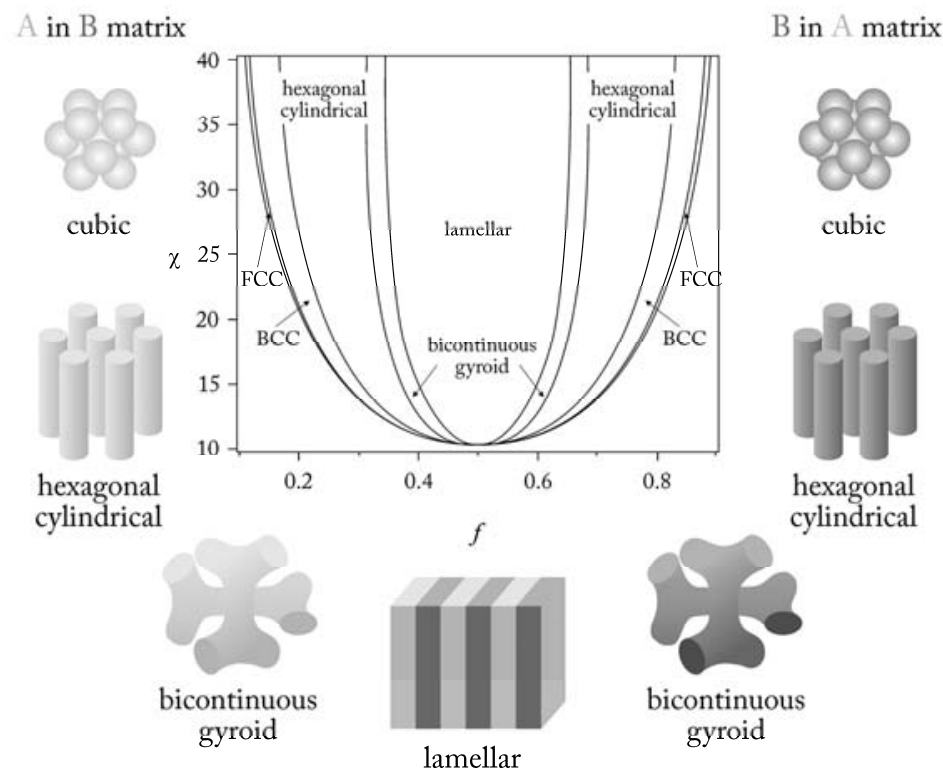

Figure 2.14 Generic phase diagram for a diblock (A-B) copolymer illustrating several of the possible domain structures (lamellar, bicontinuous gyroid, hexagonal cylindrical, cubic), as a function of the volume fraction and degree of polymer incompatibility (in terms of the Flory-Huggins parameter $\chi$ ).

Two additional factors that affect the domain structure of resulting patterns in thin films of diblock copolymers are the polymer-air and polymer-substrate interactions and the film thickness relative to the natural domain period of the bulk due to the $2 \mathrm{D}$ confinement in thin films. Systematic control over the domain structure and lateral dimensions by these parameters illustrates the potential of diblock copolymers for nanofabrication with respect to $\mathrm{CD}$ control. ${ }^{[79]}$

Two intrinsic difficulties concerning the application of BCPs for large-scale nanofabrication are the inherent short-range lateral order and the unfavorable domain orientation with respect to the substrate. Strategies for long-range lateral and orientational order are found in film thickness control, ${ }^{[80]}$ interfacial interaction control, ${ }^{[81]}$ and application of external fields such as electrical fields. ${ }^{[82]}$ These strategies, in combination with conventional lithography, allow the precise alignment of the overlaying BCP patterns on substrates and spatial control of the domain patterns. ${ }^{[83]}$ Additionally, the incorporation of multiple functionalities into the components of the BCP facilitates the spatial control by large-scale patterning. The most interesting example comprises the incorporation of high-resolution poly(4hydroxystyrene) (PHS) and poly( $\alpha$-methylstyrene) (PaMS) into one BCP and multistep patterning with DUV lithography. ${ }^{[84]}$ 
$\mathrm{BCP}$ nanostructures serve in most applications as templates in the fabrication of functional nanostructures. The primary means of achieving these nanoporous templates is by selective removal of one of the components of the BCP by chemical etching, ${ }^{[85]}$ selective degradation, ${ }^{[85]}$ or chemical modification (e.g., crosslinking ${ }^{[86]}$ ). Owing to developments in $\mathrm{BCP}$ chemistry, novel high-resolution Fe-containing ${ }^{[87]}$ and Si-containing ${ }^{[88]} \mathrm{BCPs}$ are available with larger etching contrasts within the $\mathrm{BCP}$. As shown in Fig. 2.15, the extremely high feature density of BCP templates (up to $\sim 10^{11}$ holes $\left.\cdot \mathrm{cm}^{-2}\right)^{[85]}$ makes BCP lithography useful for the fabrication of a variety of functional nanostructures, including magnetic storage media, ${ }^{[87]}$ PDMS stamps, ${ }^{\left[{ }^{[8]}\right.}$ semiconductor capacitors, ${ }^{[00]}$ quantum dots (Fig. 2.15a), ${ }^{[91]}$ nanoparticles, ${ }^{[92]}$ nanowires (Fig. 2.15b), ${ }^{[93]}$ nanotubes, ${ }^{[94]}$ nanopillars, ${ }^{[95]}$ nanoporous materials ${ }^{[85,96]}$ and in particular photonic crystals. ${ }^{[97]}$ As shown in Figs. 2.14 and $2.15 \mathrm{c} / \mathrm{d}$, BCPs have the capability to form 1D (lamellar), 2D (hexagonal cylindrical), and 3D (bicontinuous gyroid) photonic crystals and are therefore extremely useful as photonic band gap (PBG) materials. ${ }^{[98]}$
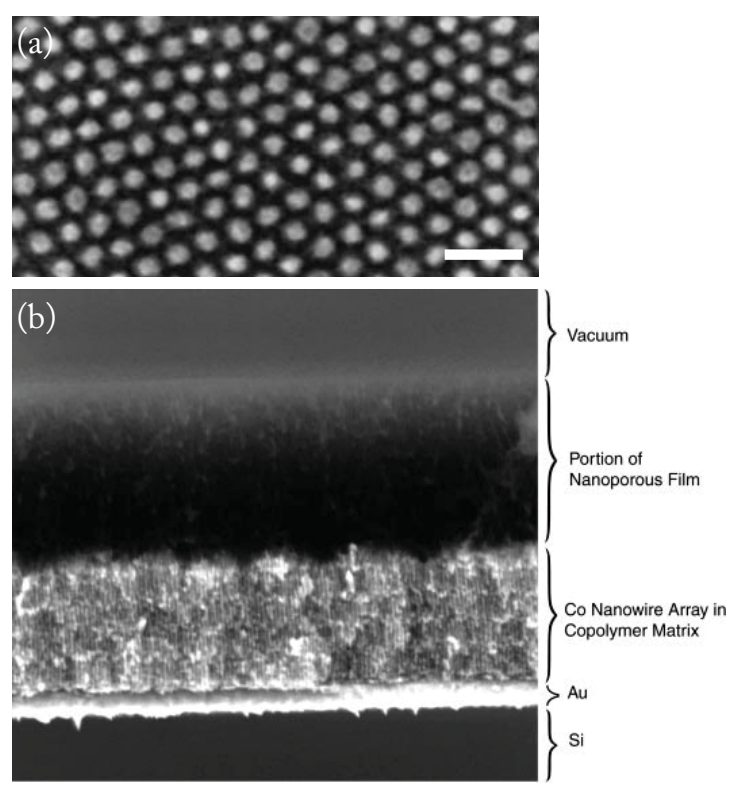
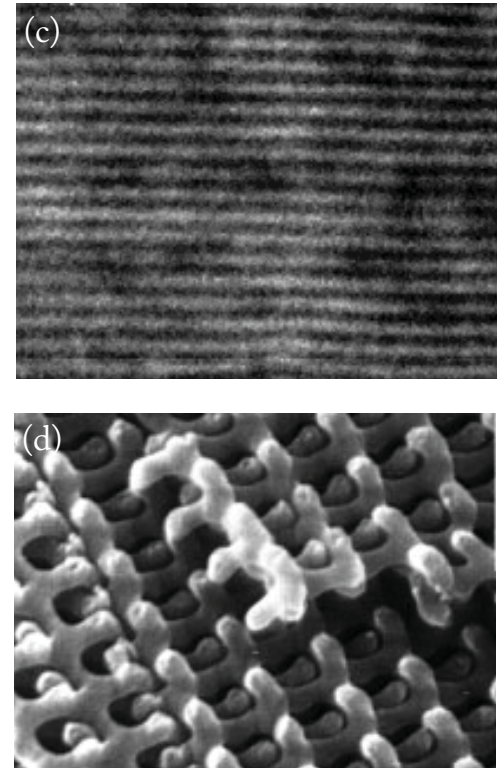

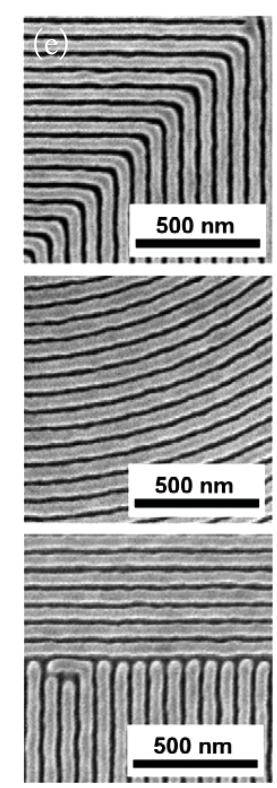

Figure 2.15 Several examples of BCP lithography in the fabrication of different functional nanostructures: (a) SEM image of a dense array of GaAs quantum dots with a mean lateral dimension of $23 \mathrm{~nm}( \pm 3 \mathrm{~nm})$ (scale bar: $100 \mathrm{~nm}$ ), ${ }^{[91]}$ (b) cross-sectional SEM image of a dense array of Co nanowires within a block copolymer matrix, ${ }^{[93 a]}(\mathrm{c} / \mathrm{d})$ SEM images of photonic band gap materials, a lamellar structure of a poly(styrene-bisoprene) film (molecular weight of $390 \mathrm{kDa}$, symmetric block copolymer, including $40 \%$ of homopolymer) with a periodicity of $140 \mathrm{~nm}^{[99 a]}$ and bicontinuous gyroid structure of another poly(styrene-b-isoprene) film (molecular weight of $300-450 \mathrm{kDa}$ and $38 \%$ of poly(styrene)) after selective UV etching of isoprene, resulting in freestanding styrene networks. ${ }^{[996]}$ (e) Series of non-regular structures: $90^{\circ}$ bends, arcs, and T-junctions. ${ }^{[79 a]}$ 
Preferential sequestering of optically transparent nanoparticles (e.g., CdSe nanoparticles) into one of the BCP domains can substantially increase the intrinsic small dielectric contrast within the BCP, resulting in larger band gaps. ${ }^{[100]}$ The practical short wavelength limit for polymer PBG materials is around $300 \mathrm{~nm}$ due to the strong absorption in the ultraviolet regime. The ease of tailoring the domain size (on the order of $10-50 \mathrm{~nm}$ ) of BCP domain patterns to $\lambda / 4 \mathrm{n}$ (with $\mathrm{n}$ the refractive index of the domain material) by the molecular weight as well as by addition of homopolymers or selective non-volatile solvents allows the practical extension of BCP PBG materials to the visible region. ${ }^{[101]}$

$\mathrm{BCPs}$ are also attractive for general nanolithography because of the potential improvements in CD control and line-edge roughness (LER) of the templates. The latter is in theory proportional to the degree of polymer incompatibility within the $\mathrm{BCP}$ and therefore in principal allows tailoring of the LER by polymer chemistry. ${ }^{[102]}$ Groundbreaking work in BCP lithography was shown recently in the fabrication of non-regular and complex structures (see Fig. 2.15e ${ }^{[103]}$ by blending pure block copolymers with homopolymers. The combination of resulting flexibility in pattern generation with precise CD control and low LER of these polymer templates illustrates that BCP lithography has the potential to become part of the nextgeneration lithographic techniques. ${ }^{[79 a]}$

\subsubsection{Physical self-organization of polymers}

This technique exemplifies the potential of CFL (Section 2.3.2) and concerns the capillary-driven self-organization of polymers into multilevel polymeric structures during the annealing of existing polymer microstructures in the presence of elastomeric stamps. ${ }^{[104]}$ This strategy overcomes the requirement of surface modification procedures, as in the case of (anisotropic) spinodal dewetting. ${ }^{[105]}$ Fig. 2.16a illustrates the time-line during the formation of the most interesting structures by capillary dynamics. By rotating the stamp $90^{\circ}$ with respect to the polymer template, holes form in the void spaces in between the elastomeric mold and the polymeric pattern owing to the formation of two menisci within a certain time interval. 

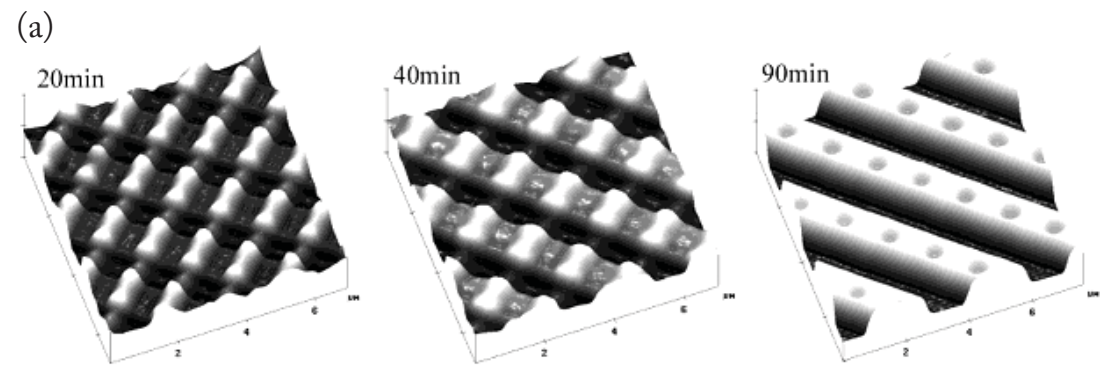

(b)
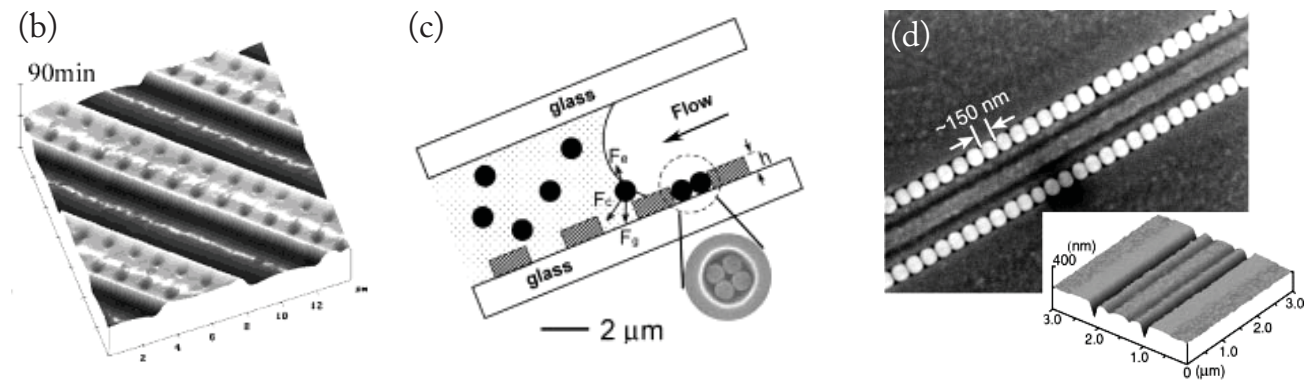

Figure 2.16 (a) AFM images of transient polymeric structures as a function of annealing time $\left(130{ }^{\circ} \mathrm{C}\right)$ using molds with a $1 \mu \mathrm{m}$ line-and-space pattern on top of a $700 \mathrm{~nm}$ line-and-space polymeric structure (height scale $300 \mathrm{~nm})^{[104 a]}$ (b) AFM image of the resulting structure in case of using molds with a $3 \mu \mathrm{m}$ line-and-space pattern (height scale $500 \mathrm{~nm}$ ). ${ }^{[104 a]}$ (c) Schematic illustration of the assembly process for generating aggregates of nanoparticles under physical confinement of the polymeric template, using the interplay of several forces acting on the nanoparticles during the assembly process: capillary force $\left(F_{c}\right)$, gravitational force $\left(F_{g}\right)$, and electrostatic force $\left(F_{e}\right) \cdot{ }^{[107]}(d)$ SEM image of two linear chains of $150 \mathrm{~nm}$ PS particles on a polymeric template made by optical near-field phase-shifting lithography (see Section 2.2.1.1). ${ }^{[107]}$

As shown by the transient structures in Fig. 2.16a, the second meniscus starts to form at the time the elastomeric mold contacts the surface of the substrate (in this particular case, at $40 \mathrm{~min}$ ) and depending on the lateral dimensions of the mold with respect to the half-width of the polymer meniscus, capillary dynamics can result in one or two rows of small holes (Figs. 2.16a/b). The lateral dimensions of these holes are about one third of the channel width of the mold and monotonically decrease at higher annealing temperatures. By performing this procedure on silicon-on-insulator, one could generate a mask for the fabrication of 1D photonic waveguides in silicon by successive etching of the substrate. ${ }^{[106]}$ Additionally, these multilevel polymeric structures are useful to act as physical templates to direct the assembly of single nanoparticles (Figs. 2.16c/d) ${ }^{[107]}$ and the multilayer assembly of functional polymers. ${ }^{[108]}$ 
patterning principles

\subsubsection{Nanosphere lithography}

The easy access of large quantities of monodisperse colloids has been a stimulant for exploiting the self-assembly of colloidal aggregates ${ }^{[109]}$ as building blocks for the generation of systems with complexities that are useful for photonic applications. ${ }^{[110,111]}$

Nanosphere lithography (NSL) ${ }^{[34]}$ exploits the self-assembly of colloids in the costeffective generation of mono- and bilayer colloidal crystal masks for various postprocessing steps, including metal deposition, ${ }^{[34]} \mathrm{SAM}$ formation ${ }^{[112]}$ and reactive ion etching. ${ }^{[113]}$ After removal of the colloids by lift-off, large-area arrays of functional nanostructures are left on the surface of the substrate. For NSL, most contributions exploit the resulting metal nanostructure arrays after metal deposition as templates in the transformation to metal nanoparticles by thermal annealing, ${ }^{[114]}$ as catalysts in the generation of silicon nanopillars by molecular beam epitaxy, ${ }^{[115]}$ carbon nanofibers $(\mathrm{CNFs})^{[116]}$ and $\mathrm{ZnO}$ nanopillars ${ }^{[117]}$ by chemical vapor deposition (CVD), as etch masks in the fabrication of nanoimprint molds ${ }^{[118]}$ and $2 \mathrm{D}$ photonic crystal patterns $^{[119]}$ by reactive ion etching, and as wet-etch masks in the generation of silicon nanopillars with different orientations by successive anisotropic silicon etching. ${ }^{[120]}$
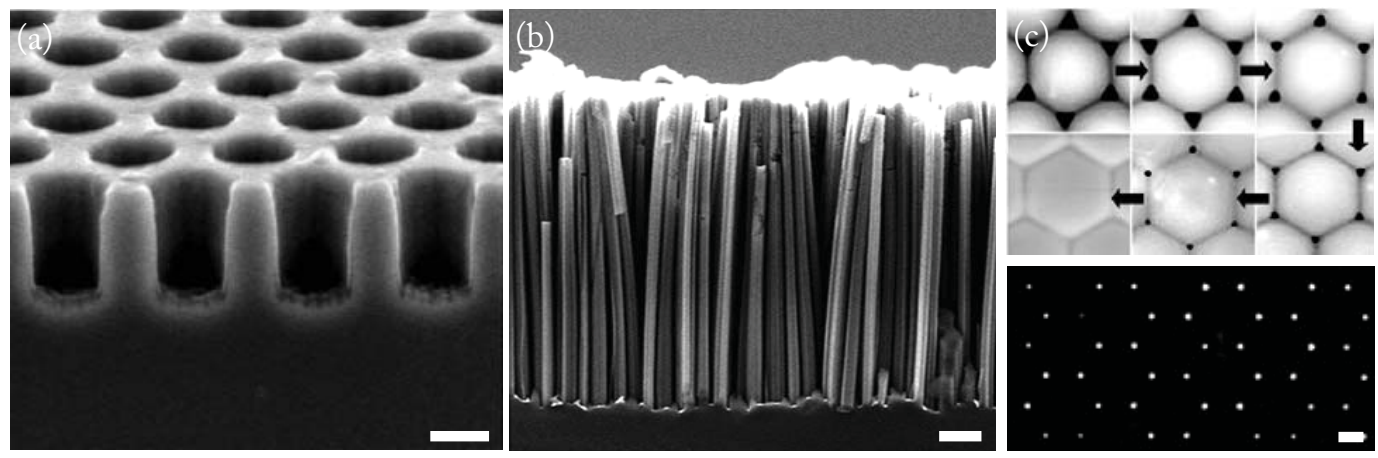

Figure 2.17 Several examples of NSL in the fabrication of different functional nanostructures: (a) SEM image of 2D photonic crystal patterns (scale bar: $250 \mathrm{~nm}$ ), ${ }^{[119]}$ (b) cross-sectional SEM image of silicon nanowires (SiNWs) after etching in HF using the silver pattern as a catalyst (scale bar: $1 \mu \mathrm{m}$ ). ${ }^{[120]}$ (c) Effect of thermal annealing of colloidal poly(styrene) particles by microwave pulse heating in water/ethanol/acetone (3:1:1) on the dimensions of the aperture in between the particles and SEM image of the resulting array of Co magnetic particles by deposition with lateral dimensions down to $30 \mathrm{~nm}$ (scale bar: $200 \mathrm{~nm}$ ). ${ }^{[121 b]}$

Control over the lateral dimensions and the periodicity of the metal structure arrays is feasible by $\mathrm{O}_{2}$ plasma, ${ }^{[113]}$ thermal annealing, ${ }^{[121]}$ or ion polishing ${ }^{[122]}$ and by 
adjusting the diameter of the colloidal particles, respectively. According to computer simulations, ${ }^{[121]}$ it is within the capability of NSL to generate several different feature shapes by adjusting the relative position of the evaporation source during deposition. However, with the restriction that clogging of the nano-apertures limits the total time accessible for metal deposition. Although NSL offers large-area patterning in a parallel fashion, on the macroscopic scale this technique remains susceptible to defects, distortions and grain boundaries that form during the self-assembly of colloidal particles on surfaces.

\subsubsection{Pattern formation by anodic oxidation (anodization)}

The electrochemistry of elements ${ }^{[123]}$ offers possibilities for the generation of nanoscale patterns in a large set of materials. Since the growing demand for highresolution templates to fabricate magnetic, electronic and photonic devices, the anodic oxidation (or anodization) of thin aluminum films in acidic solutions ${ }^{[24]}$ has received considerable interest during the last 25 years owing to the formation of high-density arrays (up to $10^{11}$ holes $\cdot \mathrm{cm}^{-2}$ ) of cylindrical nanopores in a triangular lattice in anodic aluminum oxide (AAO) films (Fig. 2.18). ${ }^{[125]}$

(a) OXIDE
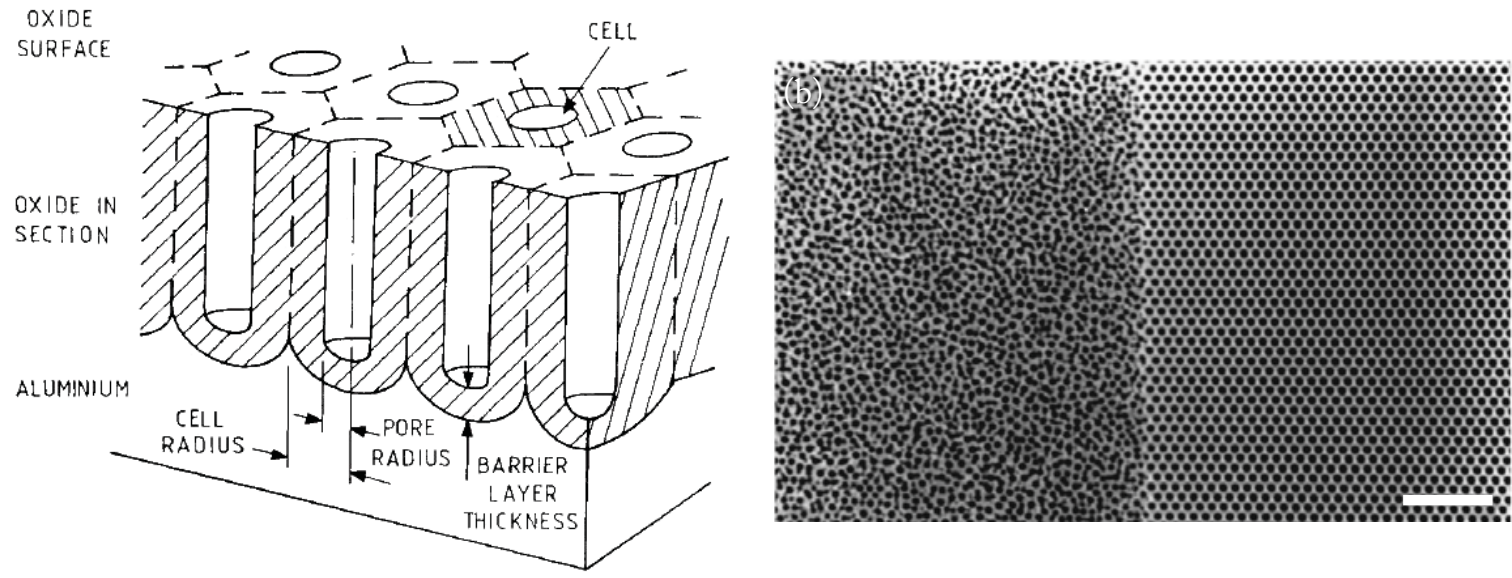

Figure 2.18 (a) Configuration diagram of porous AAO membranes. The hexagonal array of cells contains a cylindrical pore in the center of each cell. These pores are perpendicular to the surface of the aluminum film and extend down to a non-porous aluminum oxide barrier layer. The pore diameter is between one third and half of the lateral dimensions of the cell. Pore widening by etching in acidic solutions is optional to fine-tune the lateral dimensions of the pore. ${ }^{[124]}$ (b) SEM images illustrating the effect of pre-texturing by means of a hard $\mathrm{SiC}$ mold on the formation of defect-free AOO membranes over surface areas on the order of millimeters (scale bar: $1 \mu \mathrm{m}) \cdot{ }^{[127 \mathrm{~b}]}$ 
The balance during the anodization process of the AAO membranes between oxide dissolution at the electrolyte/oxide interface and oxide formation at the oxide/ aluminum interface is crucial for steady-state pore propagation into the aluminum film. ${ }^{[126]}$ Fundamental research on the pore formation suggests that repulsive forces at the oxide/aluminum interface between adjacent pores promote the self-organization of the pores into hexagonal arrays. ${ }^{[126]}$ As the regularity of the pores and pore arrangement increase with time, the resulting membranes in a conventional one-step anodization process are far from perfect. A two-step anodization process, ${ }^{[127 a]}$ annealing and electropolishing of the aluminum layer, and pre-texturing the aluminum film by imprinting with hard molds ${ }^{[127 b]}$ are measures taken to overcome the irregularities of the conventional anodization process for the generation of defect-free AAO membranes over large areas (Fig. 2.18b).

Precise control of the pore dimensions in the range of 10-200 nm (with corresponding cell dimensions of $25-420 \mathrm{~nm}$ ) is feasible by adjusting the electrolyte composition (sulfuric acid for small-pore membranes $(10-30 \mathrm{~nm})$, oxalic acid for medium-pore membranes $(30-80 \mathrm{~nm})$, and phosphoric acid for large-pore membranes $(>80 \mathrm{~nm})$ ), anodization voltage and temperature. ${ }^{[124]}$ The extent of this control and the regularity of the patterns illustrate the applicability of the AAO membranes as excellent templates in the fabrication of arrays of one-dimensional nanostructures, such as nanowire and nanotube arrays of metals, ${ }^{[128]}$ semiconductors, ${ }^{[129]}$ carbon, ${ }^{[130]}$ and replication into metal membranes, ${ }^{[131]}$ PDMS stamps ${ }^{[132]}$ and imprint molds. ${ }^{[133]}$

The development of ultra-thin AAO membranes (with thicknesses of about several hundreds of nanometers) overcomes the difficulties of the conventional AAO membranes to fabricate zero-dimensional nanostructures (e.g., nanoparticles and nanoholes) directly on the substrate due to the oxide barrier layer and aluminum backside. Owing to the small aspect ratio of the pores, these (freestanding) membranes $^{[134]}$ are excellent deposition and etch masks for the fabrication of semiconductor, oxide and metal nanoparticle ${ }^{[135]}$ and nanohole $\mathrm{e}^{[136]}$ arrays, respectively, and structures from processes that initiate from nanoparticle arrays onto any surface (e.g., nanotubes ${ }^{[137 \mathrm{a}]}$ and nanopore arrays $\left.{ }^{[137 \mathrm{~b}]}\right)$.

Fig. 2.19 illustrates the general applicability of AAO templates. Several contributions integrate the $\mathrm{AAO}$ templates in the fabrication of functional structures 
by conventional lithography, ${ }^{[138]}$ e.g., by the FIB sputtering of AAO templates for the selective closure of part of the nanochannels. ${ }^{[1386]}$ As in the case of BCP lithography (Section 2.2.3.1), the ultra-high density of the AAO templates opens up the possibility to fabricate photonic crystal devices with this technique (Fig. 2.19c). ${ }^{[139]}$

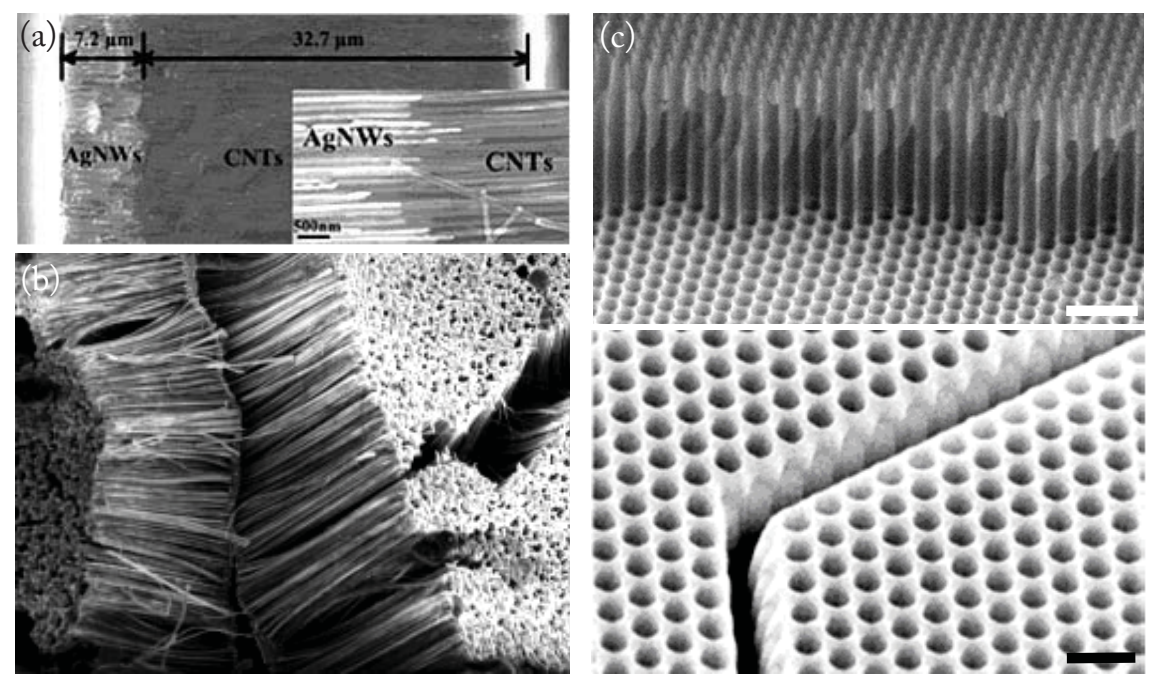

Figure 2.19 Several examples of AAO membranes in the fabrication of different functional nanostructures: (a) cross-sectional SEM image of a $\mathrm{AgNW} / \mathrm{a}-\mathrm{CNT}$ heterojunction array within the AAO membrane, ${ }^{[140]}$ (b) SEM image of uniform metal nanotube arrays after dissolution of the AAO template and the metal core, ${ }^{[128 c]}$ (c) SEM images of a GaAs substrate after dry etching using the AAO template as the etch mask (scale bar: $1 \mu \mathrm{m}$ ), and the resulting 2D photonic crystal waveguide after writing of the line-defect by FIB (scale bar: $500 \mathrm{~nm}) .{ }^{[139]}$

The tunable structural parameters of the AAO membranes, the cost-effective large area patterning capability and the general applicability in the fabrication of different types of nanostructures for a range of materials makes this also a potential technique for nanofabrication.

\subsubsection{Summary}

The current set of techniques in this section is capable of structuring a wide range of materials at the sub-100 $\mathrm{nm}$ scale in a parallel and cost-effective fashion for many potential applications. The degree of development of these techniques differs to a large extent and this is essentially dependent on the potential of generating arbitrary structures. On the one side there is the set of edge lithography techniques, in principle incapable of generating arbitrary features. The high-resolution features are, however, very useful in the cost-effective fabrication of nanowires and nanochannels. On the 
other side there are DPN and (to a less extent) BCP lithography, capable of generating arbitrary structures. A lot of research is done for these particular techniques to meet the stringent requirements of IC manufacturing in terms of feature perfection $(\mathrm{CD}$ and defect control), registration (overlay) and integration into complete circuit layouts. For this reason, the convergence of several unconventional techniques with or without the interference of conventional lithography is foreseen in the fabrication of complete IC layouts.

\subsection{Replication of patterns}

Pattern replication is the cost-effective reproduction of structural information present on the surface of a master into a different material, and with high fidelity. The generation of high-resolution masters with serial direct-write technologies, e.g., electron- and ion beam lithography, is only a practical protocol for high-throughput IC manufacturing in combination with a cost-effective and high-throughput replication technique. This section presents a selection of the most powerful techniques in unconventional nanofabrication for replicating high-resolution $(<100 \mathrm{~nm})$ features of a master.

\subsubsection{Pattern replication by imprinting}

Nanoimprint lithography (NIL) is the revelation of the 90s for high-throughput patterning of polymers ${ }^{[141]}$ owing to its low capital costs and potential in highresolution patterning, as shown by the demonstration of $10 \mathrm{~nm}$ feature reproduction with a parallel-plate press soon after its inception. ${ }^{[142]}$ Since 2003 , NIL is part of the emerging technologies of the ITRS for future IC manufacturing at the 32-nm node and beyond (Fig. 2.2). The concept of thermal NIL is the compression molding of a thin polymer film cast on a substrate with a hard mold at temperatures above the glasstransition temperature $\left(T_{g}\right)$, in order to force the polymer to conform to the nanoscale surface-relief features of the mold (Fig. 2.20). For most applications, anisotropic $\mathrm{O}_{2}$ plasma etching is essential to remove the polymer residual layer and therefore enable successive processing of the substrate. A lot of fundamental research can be found in literature addressing the critical and industrially relevant process issues of thermal NIL due to the mechanical nature of the process (Table 2.3). 
(a)

Imprint
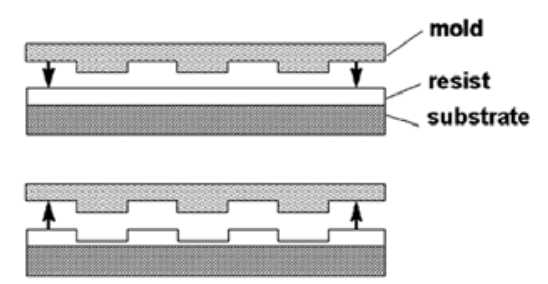

Pattern Transfer

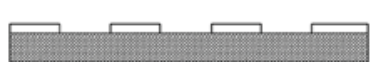

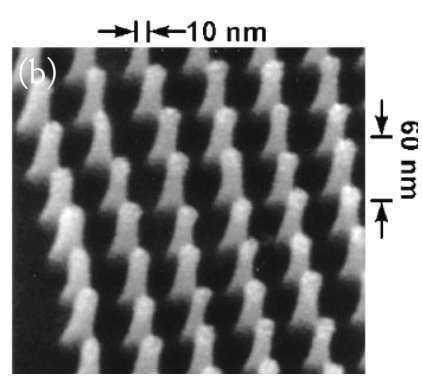

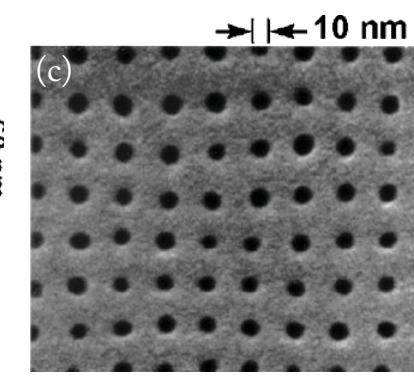

Figure 2.20 (a) Schematic representation of thermal nanoimprint lithography. (b, c) SEM images of a hard mold with a high-density array of pillars with lateral dimensions of $10 \mathrm{~nm}$ and the corresponding imprint in poly(methylmethacrylate) (PMMA). ${ }^{[142]}$

As shown in Table 2.3, considerations for selecting mold materials include hardness (for preservation of the nanoscale features), durability (for high-throughput), thermal expansion coefficient (for accurate registration and pattern fidelity during imprinting at high temperatures), and compatibility with IC manufacturing processes (for production of the molds).

Table 2.3 List of process issues in thermal imprinting

\begin{tabular}{|c|c|}
\hline $\begin{array}{l}\text { Molding/ } \\
\text { demolding }\end{array}$ & $\begin{array}{l}\text { Processing cycle time: fast heating systems with negligible resist cooling times by } \\
\text { application of heat to the stamp }{ }^{[143]} \text { or of a single laser pulse, }{ }^{[144]} \text { room temperature } \\
\text { systems, }{ }^{[145,146,147]} \text { and pressure control, }{ }^{\left[148,{ }^{*}\right]} \\
\text { demolding implications }{ }^{[149]} \text { and incorporation of step-and-repeat systems } \\
\text { presence of residual layer }{ }^{[150 a]} \\
\text { multilevel processing (alignment) } \\
\text { contamination control: intermediate polymer stamps }(\mathrm{IPS})^{[152]} \\
\text { real-time monitoring systems }{ }^{[153]} \text { and feature analysis }{ }^{[150 b]}\end{array}$ \\
\hline Resist & $\begin{array}{l}\text { visco-elastic properties }^{[154]} \\
\text { etch resistance and selectivity } \\
\text { thermal stability during thermal imprinting and mechanical stability during } \\
\text { demolding }[155,156]\end{array}$ \\
\hline Mold & $\begin{array}{l}\text { hardness, durability and surface energy control: anti-adhesive coatings }{ }^{[157,158]} \text { and novel } \\
\text { anti-adhesive mold materials }{ }^{[147,159,160]} \\
\text { mold design rules for optimal filling }{ }^{[161]} \\
\text { implications by difference in thermal expansion coefficient between substrate/mold }{ }^{[162]}\end{array}$ \\
\hline Transfer & pattern transfer fidelity of plasma etching ${ }^{[163]}$ \\
\hline
\end{tabular}

* Several efficient strategies to reduce the imprint pressure in nanoimprint lithography are part of soft lithography (see Section 2.3.2). 
Several materials that are eligible for thermal NIL are $\mathrm{Si}, \mathrm{SiO}_{2}, \mathrm{SiC}$, silicon nitride, and metals. The stringent material aspects for molds, however, do not exclude the use of composite molds consisting of flexible backplanes supporting thin molds to combine global flexibility with local hardness of the nanoscale features ${ }^{[164]}$ and the use of transparent molds (quartz, silica) for room-temperature NIL of UV-curable resists (UV-NIL). ${ }^{[165]}$

Generally, the total surface area of high-resolution molds is known to cause strong adhesion of the resist at the mold during imprinting and results in severe damage to the resist patterns. Solutions to reduce this adhesion and increase the mold durability are (1) incorporation of internal release agents in resist formulations, (2) application of low-surface energy coatings (in thin film ${ }^{[157]}$ or monolayer ${ }^{[158]}$ of surfactants) to the mold, and (3) fabrication of a mold from materials with intrinsic low surface energy. ${ }^{[147,159,160]}$

The ideal resist formulations for NIL have several characteristic properties for successful pattern transfer, including good deformability at the imprint conditions (pressure, temperature, and viscosity), sufficient mechanical strength and excellent mold-releasing properties to maintain the structural integrity of the polymeric features during demolding, and superior etching properties for successive pattern transfer into the substrate with high fidelity. Practically, imprint temperatures that are $70-90{ }^{\circ} \mathrm{C}$ above $T_{g}$ of the thermoplastic polymer are chosen to ensure sufficient polymer flow. ${ }^{[166]}$ For reasons of high-throughput, current research focuses on approaches that lower the total processing time of imprinting and includes the development of new high-resolution resist formulations (other than the standard PMMA resist) that are more suitable for nanoimprint applications. The low structural integrity of resist features from resist formulations with a low $\mathrm{T}_{\mathrm{g}}$ infer that thermally curable liquid resists are a good option for low-pressure imprinting that result in features with good structural integrity owing to thermal crosslinking. A very recent thermally curable resist $^{[167]}$ makes use of the hydrosilylation chemistry of siloxane polymers and consists of four basic components (PDMS precursor, crosslinker, catalyst and inhibitor). The inhibitor keeps the catalyst inactive and in combination with the low viscosity of the resist allows low-pressure $(0.2-0.6 \mathrm{MPa})$ imprinting at room temperature down to a resolution of $70 \mathrm{~nm}$. Two additional features of this resist is the very fast curing time 
of $10 \mathrm{~s}$ upon heating the system to $120^{\circ} \mathrm{C}$ and the ability to tune the modulus by the molecular weight of the PDMS precursor and the reactive groups in the crosslinker. Siloxane block or graft copolymers ${ }^{[168]}$ also form a potential class of (in this case) thermoplastic polymers for NIL (conditions: $170{ }^{\circ} \mathrm{C}, 1.3 \mathrm{MPa}$ ) by providing dual surface properties owing to the phase separation at annealing (Section 2.2.3.1). This dual surface property results in superior mold-release performance without delamination from the substrate (impossible for homopolymer resists). For certain (thermo-sensitive) applications, UV-curable epoxysilicone resists ${ }^{[147]}$ have recently become available for room temperature imprinting and overcome the oxygen sensitivity and large shrinking problems during curing of conventional acrylic UVcurable resists. Owing to the high silicon content, the three new resist formulations possess excellent etch resistance to facilitate successive pattern transfer into the substrate by plasma processing. A last potential inorganic resist formulation for room temperature imprinting is one of several spin-on-glass types, hydrogen silsesquioxane (HSQ). ${ }^{[145]}$

Real-time imprint monitoring systems (RIMS) ${ }^{[153]}$ are under development that allow ultimate control over the imprinting process (temperature, pressure, time). Fig. 2.21 illustrates the capability of RIMS for monitoring imprint processes on the nanosecond scale.

(a)

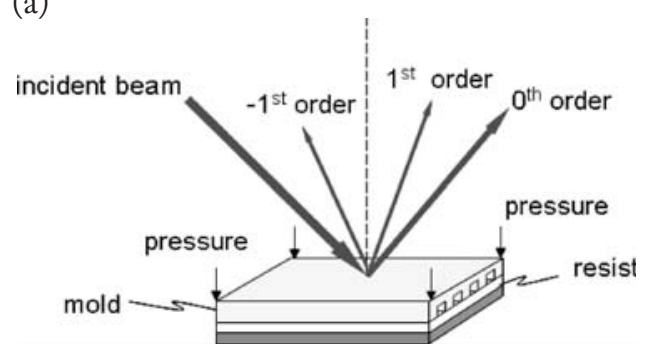

(b)

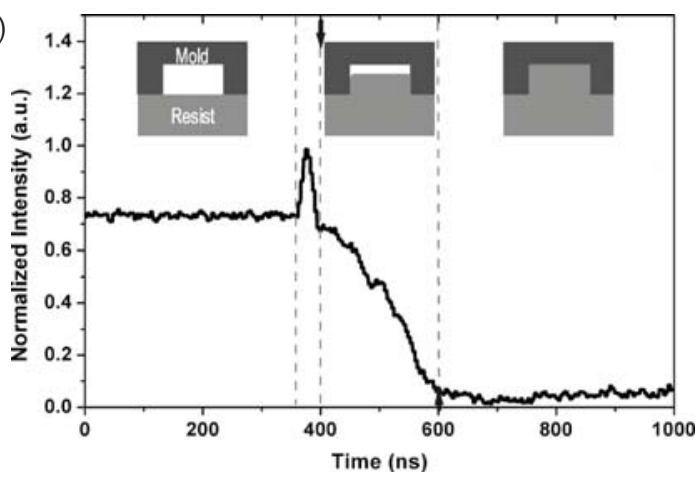

Figure 2.21 (a) Schematic of the measurement set-up for RIMS. The laser beam is incident on the mold and an ultrafast photodetector is collecting the negative first order diffraction signal for analysis by an oscilloscope. (b) Typical curve illustrating the intensity change as a function of time, with the blue arrows indicating the start and end point of the imprint. ${ }^{[153]}$

The condition for short processing times in IC manufacturing has driven current research activities in thermal NIL to step-and-repeat patterning schemes that have a 
strong similarity with the stepper system in conventional photolithography. These schemes concern the lateral confinement of heat transfer by application of a single laser pulse with a suitable wavelength as the heat source in order to melt the surface of polymeric $^{[143 a]}$ as well as hard materials (e.g., silicon) ${ }^{[143 b]}$ in less than 1 ns, allowing ultrafast imprinting times of about 200 ns (Fig. 2.21b). Additional advantages of these heating sources are the negligible resist cooling time and reduction of thermal expansion effects.

A very illustrative procedure in literature that has close operational similarities to microtransfer molding ${ }^{[169]}$ and that aims for enhancing the patterning capability is reverse NIL ${ }^{[170]}$ of true 3D patterns (Fig. 2.22). For reverse nanoimprint lithography, the successful transfer depends on the balance of the surface energies between the mold and the substrate and can result in two different reversal imprint modes. ${ }^{[171]}$ Analogs in GaAs of the 3D structure in Fig. 2.22 form potential photonic structures to control the emission of light. ${ }^{[172]}$
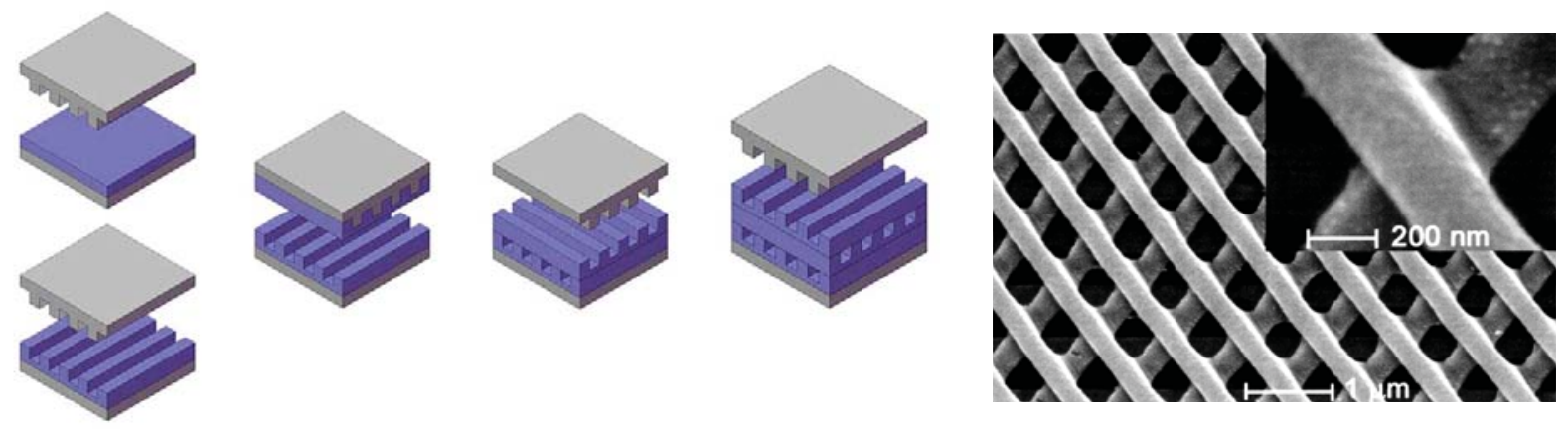

Figure 2.22 Schematic representations of multi-level patterning by reverse nanoimprint lithography and a representative SEM image of a true 3D periodic structures consisting of a stack of three consecutive polymer structures by building up in a layer-by-layer fashion. ${ }^{[170 a]}$

Although research and development still need to address some of the important issues for integration in IC manufacturing, Fig. 2.23 illustrates that NIL has already found numerous applications. These applications include electronics (e.g., hybrid plastic electronics, ${ }^{[173 a]}$ organic thin-film transistors, ${ }^{[174]}$ silicon nanoelectronic devices $\left.{ }^{[175]}\right)$, photonics (e.g., organic near-IR lasers, ${ }^{[176]}$ nonlinear optical polymeric nanostructures, ${ }^{[177]}$ high-resolution organic light-emitting diodes (OLEDs) ${ }^{[178]}$, and 
high-performance polarizers ${ }^{[179]}$ ), magnetics (e.g., high-density magnetic media ${ }^{[180]}$ ), and biological applications (e.g., manipulation of DNA in nanofluidic channels, ${ }^{[181]}$ nanoscale protein patterning $\left.{ }^{[182]}\right)$.
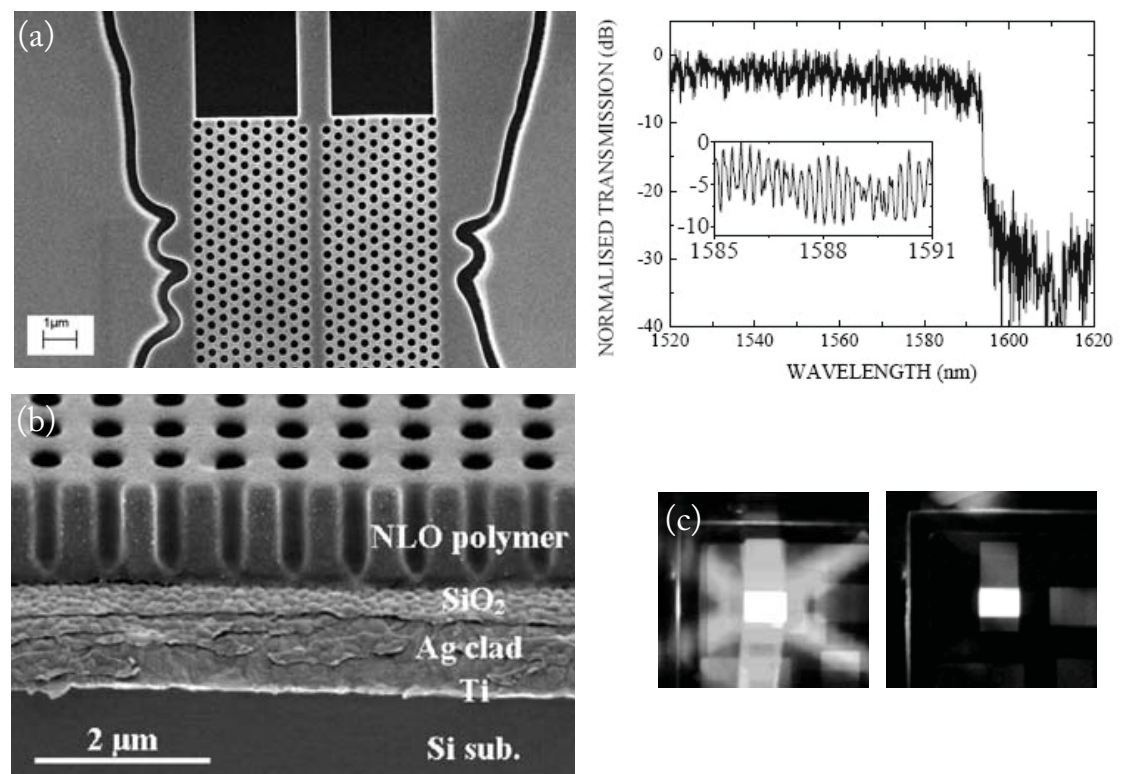

Figure 2.23 Several examples of photonic nanostructures by application of nanoimprint lithography: (a) SEM image of a $10 \mu \mathrm{m}$-long $2 \mathrm{D}$ photonic crystal waveguide in SOI and a transmission spectrum for quasi-TE laser light, ${ }^{[183]}$ (b) SEM image of a defect-free 2D photonic crystal in a nonlinear optical polymer, ${ }^{[177 b]}$ (c) photographs of OLEDs with (left image) and without (right image) the presence of the photonic crystal. ${ }^{[178]}$

\subsubsection{Pattern replication by conformal contact (soft lithography)}

Soft lithography is a collective name for a set of non-photolithographic techniques that rely on the use of elastomeric stamps as the key element for pattern transfer. ${ }^{[30]}$ Soft lithography offers immediate advantages over conventional lithographic techniques in the structuring of materials that are incompatible with corrosive etchants, high-energy radiation and high temperatures and in the structuring of fragile or non-planar substrates by large-area conformal contact with substrates without resorting to high pressures. Several of these advantages ensue from the properties of PDMS as the elastomeric stamp. The elasticity and low surface energy of elastomeric PDMS stamps $\left(21.6 \cdot 10^{-3} \mathrm{~J} \cdot \mathrm{m}^{-2}\right)^{[184]}$ allow conformal contact over large areas and facilitate easy release from the substrate after patterning, respectively. PDMS is a durable elastomer, chemically inert to most materials, resistant to many organic solvents, ${ }^{[185]}$ optically transparent down to $\sim 280 \mathrm{~nm}$, and moreover allows the 
modification of its surface by SAMs to provide a wide range of surface energies. ${ }^{[186]}$ However, the low tensile modulus of common PDMS (Dow Corning, Sylgard 184) of 1.8 $\mathrm{MPa}$ compromises the dimensional stability of high-resolution features due to capillary forces, self-adhesion and mechanical stresses during patterning. ${ }^{[187]}$ Several formulations of PDMS with a higher tensile modulus extend the use of elastomeric stamps in high-resolution soft lithography and include thermally curable PDMS (h-PDMS) ${ }^{[188 a, b]}$ and ultraviolet (UV)-curable PDMS, ${ }^{[188 c]}$ with a tensile modulus of 8.2 $\mathrm{MPa}$ and 3.4 $\mathrm{MPa}$, respectively, and combinations thereof in the form of composite stamps. ${ }^{[188 \mathrm{~b}]}$

Table 2.4 Potential alternative stamp materials in literature for high-resolution soft lithography

Stamp material Properties

Teflon AF 2400 (DuPont) ${ }^{[159]}$

$\sigma=1.6 \mathrm{GPa}$, surface energy of $15.6 \mathrm{~J} \cdot \mathrm{m}^{-2}$, resistant to any organic solvent, 10000 times larger gas permeability than PDMS (ideal for soft molding ${ }^{[209]}$ ), polymers feature with aspect ratios $>6$ in sub-100 nm range by imprinting, transparent from deep ultraviolet (UV) and near-infrared (IR)

$\mathrm{UV}$-curable urethane acrylate pre-polymer (Ebecryl 265, SK-UCB) $)^{[189 a]}$

UV-curable polymer (Ebecryl 280 by SK-UCB) $)^{[189 b]}$

UV-curable perfluoropolyether $(\mathrm{PFPE})^{[190]}$

UV-curable NOA 63

(Norland Products) $^{[191]}$

polyolefin plastomers (POPs $)^{[192]}$ $\sigma=20-320 \mathrm{MPa}$ (tunable by type and amount of modulator) for contact printing and nanoimprinting, surface energy of $23.0 \mathrm{~J} \cdot \mathrm{m}^{-2}$, and resistant to most organic solvents

$\sigma=40 \mathrm{MPa}$, surface energy of $23.0 \mathrm{~J} \cdot \mathrm{m}^{-2}$, optional selfreplication by UV-curing, shrinkage of $0.7 \%$ during curing, and resistant to most organic solvents

$\sigma=4 \mathrm{MPa}$, higher resistance to organic solvents and lower intrinsic surface energy than PDMS

good mechanical stability, with a softening transition above $48{ }^{\circ} \mathrm{C}$ for conformal contact (ideal for polymer spin transfer printing $\left.{ }^{[203]}\right)$, transparent from 360 to $1260 \mathrm{~nm}$

fast preparation by compression molding $(\sim 1 \mathrm{~h})$, significant contact-printing performance in the sub-100 nm range, and no surface contamination during conformal contact with the substrate, as is the case with $\mathrm{PDMS}^{[193]}$

In Table 2.4, several other potential stamp materials are shown with a wide range of properties, depending on the application in high-resolution soft lithography 
(e.g., contact printing, imprinting, etc.). Any soft-lithographic technique starts with replica molding (REM) of the surface-relief features of a master into elastomeric stamps by casting and curing of the elastomeric precursor against the master. REM is capable of replicating features with very high fidelity and accuracy down to lateral dimensions of $\sim 1.5 \mathrm{~nm},{ }^{[194 \mathrm{a}]}$ i.e., the length scale of large molecules. ${ }^{[194 \mathrm{~b}]}$ Additionally, the advantages of REM in soft lithography are numerous, from the procedural simplicity to the capability of generating multiple elastomeric copies from a single master.

The first set of techniques in soft lithography relies on the intimate conformal contact of elastomeric stamps with the surface of substrates for efficient transfer of matter on substrates. The first technique, microcontact printing, exploits elastomeric stamps as ink reservoirs to transfer molecules (initially alkanethiols) that are capable of forming SAMs on the surface of various metal films ${ }^{[30]}$ and is capable of patterning large surfaces in a single impression. A lot of studies have been done on several aspects of contact printing, including the transport mechanism of alkanethiols, ${ }^{[195]}$ formation and order of SAMs on metal films, ${ }^{[36 a]}$ and different inking modes for stamps. ${ }^{[36 b]}$ This type of contact printing is an additive process and the resulting SAMs on metal films have found many applications in nanotechnology, ${ }^{[196]}$ including the use as ultra-thin resists in the selective wet-chemical etching of metal films ${ }^{[197 \mathrm{a}]}$ and as passivating layers in selective deposition. ${ }^{[197 b]}$ Additionally, microcontact printing is extremely useful for the direct patterning of a wide range of interesting species, such as colloidal particles, ${ }^{[198 \mathrm{a}]}$ proteins, ${ }^{[198 \mathrm{~b}]}$ organic reagents, ${ }^{[198 \mathrm{c}]}$ catalysts, ${ }^{[198 \mathrm{~d}]}$ dendrimers, ${ }^{[198 \mathrm{e}]}$ nanoparticles, ${ }^{[38]}$ and polymers ${ }^{[198 f]}$ on a range of surfaces. Even with the application of resolution enhancement approaches, ${ }^{[199]}$ composite stamps ${ }^{[188 b]}$ and low-diffusion inks, ${ }^{[37]}$ the resolution of microcontact printing encounters practical limits at about $100 \mathrm{~nm}$ due to surface diffusion of molecular inks and the isotropic nature of etching and deposition systems that convert SAM patterns into patterns of functional materials.

Recent research in soft lithography has come up with several potential contactprinting techniques that circumvent the intrinsic limitations of microcontact printing and include the direct patterning of organic and inorganic on surfaces at sub-100 nm resolution (Table 2.5). As shown in Table 2.5, these direct contact-printing techniques 
are the result of ingenious engineering of the adhesion forces at the stamp/film and the film/substrate interfaces to facilitate the additive or subtractive transfer of matter by means of conformal contact of elastomeric stamps with substrates. The correct preparation of the stamp and the substrate is of critical importance, as shown in the case for the preparation of metal layers on elastomeric stamps by metal deposition without substantial formation of nanocracks that can deteriorate the integrity of the final nanostructures. ${ }^{[200]}$ nTP and MTP exploit the intrinsic low adhesion of metals (in particular gold) to PDMS and concentrate only on interfacial adhesion to the substrate.

Table 2.5 List of potential contact-printing techniques for high-resolution soft lithography

\begin{tabular}{|c|c|c|}
\hline Technique* & Type & Patterning principle \\
\hline nTP ${ }^{[201]}$ & additive & $\begin{array}{l}\text { transfer of metal patterns to surfaces by covalent bonding between metal } \\
\text { and SAMs or by surface condensation between surface silanols ( } \mathrm{Si}-\mathrm{OH}) \\
\text { and titanols ( } \mathrm{Ti}-\mathrm{OH}) \text { from the adhesion layer }\end{array}$ \\
\hline $\operatorname{MTP}^{[202]}$ & additive & $\begin{array}{l}\text { transfer of metal patterns by the formation of intimate junctions between } \\
\text { metal and polymer films upon annealing }\end{array}$ \\
\hline $\mathrm{STP}^{[203]}$ & additive & $\begin{array}{l}\text { transfer of polymer patterns by selective surface functionalization of the } \\
\text { stamp }^{[203 \mathrm{a}]} \text { or by electrostatic interactions between the polymer and the } \\
\text { polyelectrolyte adhesion promoter }{ }^{[203 \mathrm{~b}]}\end{array}$ \\
\hline DTL $^{[204]}$ & additive & $\begin{array}{l}\text { transfer of PDMS from the PDMS stamp by UV/ozone exposure } \\
\text { (oxidation) and successive cohesive mechanical failure at the surface of } \\
\text { the PDMS stamp }\end{array}$ \\
\hline $\begin{array}{l}\text { detachment } \\
\text { nanolithography }{ }^{[205]}\end{array}$ & subtractive & $\begin{array}{l}\text { transfer of polymer from the substrate by formation of intimate junctions } \\
\text { between the polymer and stamp upon annealing }\end{array}$ \\
\hline
\end{tabular}

* nTP: nanotransfer lithography; MTP: metal transfer printing; STP: spin transfer printing; DTL: decal transfer lithography.

In contrast, a systematic study on detachment lithography has shown the interplay of the intrinsic (adhesion forces and wettability) and external (temperature, pressure) parameters for the engineering of polymer detachment. ${ }^{[205 c]}$ 
There are several immediate technological advantages arising from these direct contact-printing techniques over microcontact printing, such as (1) sub-100 nm resolution, owing to the absence of surface diffusion of molecular inks or edge disorders, and (2) reduction of the number of transfer layers, owing to the higher etch resistance of polymers and metals. Additionally, the absence of a residual layer eliminates additional post-processing steps as is the case of imprinting of polymers (Section 2.3.1). The examples in Fig. 2.24 illustrate the capability of direct-contact printing techniques in the fabrication of nanostructures.
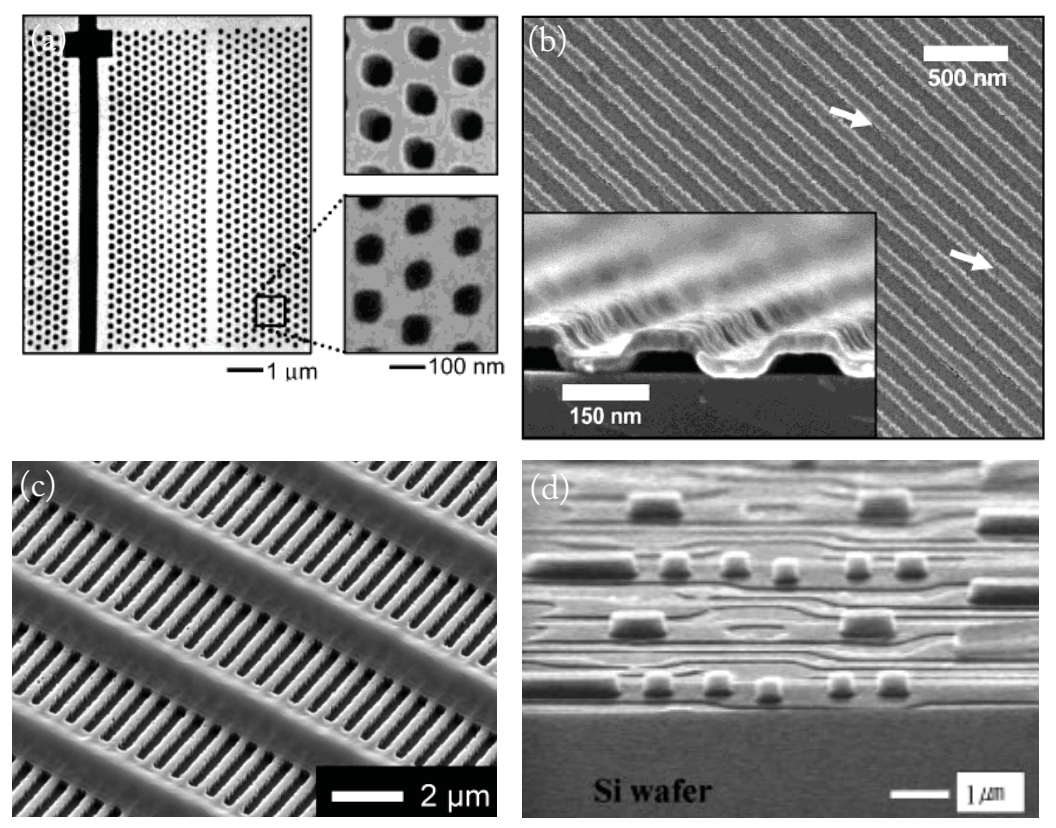

Figure 2.24 Several examples of nanostructures by contact printing: (a) SEM image of a 2D photonic waveguide pattern in gold with close-up images of the metallic PDMS stamp (top image) and of the final pattern after nTP (bottom image), ${ }^{[201 a]}$ (b) SEM images of arrays of nanochannels, ${ }^{[206]}$ (c) complex grating by two sequential application of DTL and successive reactive ion etching, ${ }^{[204 b]}$ and (d) complex polymer structures by simultaneous of physical and chemical nanostructures by STP. ${ }^{[203 b]}$

The contact-printing techniques in this section are potentially useful for the direct patterning of functional materials on delicate surfaces, for example the patterning of conductive polymers (e.g., PEDOT, m-MTDATA) for high density organic electronic circuits. ${ }^{[207]}$

The second set of techniques in soft lithography relies on the intimate conformal contact of elastomeric stamps with polymer films for efficient molding of polymers on substrates (Table 2.6). Contact-molding techniques take immediate advantage of the 
latest development of novel mold materials (Table 2.4) in the accurate replication of patterns into polymers. The combination of conformal contact over large areas and suitable surface energy of the stamp eliminates the need of high external pressures as is the case in most nanoimprint lithography schemes. CFL has the capability of generating (Sections 2.2.1.1 and 2.2.3.2) and replicating nanoscale features in polymer layers by the concept of capillary rise. The fact that contact molding of nanometer scale features by capillary rise is feasible with impermeable molds, ${ }^{[208]}$ and not on the micrometer scale, illustrates that the capillary pressure increases with decreasing feature size. For this reason the resolution of techniques using capillary rise (CFL, soft molding) seems to depend on the availability of high-resolution masters, as shown by the feature replication of $30 \mathrm{~nm} .{ }^{[208]}$ Additionally, contact-molding techniques have the capability to replicate polymer features with zero residual layer ${ }^{[29]}$ and therefore allow the direct patterning of the underlying substrate.

Table 2.6 List of potential contact-molding techniques for high-resolution soft lithography

\begin{tabular}{ll}
\hline Technique & Patterning principle \\
\hline CFL $^{[29]}$ & capillary rise of polymer melts by wetting on elastomeric stamps at temperatures above \\
& $\mathrm{Tg}$ \\
& $\begin{array}{l}\text { capillary rise of solvent-containing polymer films (i.e., spin coating or dipping without } \\
\text { baking) by wetting on elastomeric stamps at room temperature }\end{array}$ \\
& \\
soft imprint & low-pressure molding of a prepolymer/monomer film and successive UV curing \\
lithography &
\end{tabular}

An interesting technique to increase the aspect ratio of polymeric features is nanodrawing, ${ }^{[212]}$ i.e., the elongation of polymeric features upon removal of the stamp at higher temperatures (Fig. 2.24), again by engineering of the adhesive forces at the stamp/polymer interface.

An innovative development in post-processing that can significantly improve the line-edge roughness (LER) of nanostructures (of metals, semiconductors, dielectrics) was shown very recently by exploiting self-perfection by liquefaction (SPEL). ${ }^{[213]}$ SPEL exploits the use a single laser pulse for the selective melting of nanostructures on 
the timescale of hundreds of nanoseconds in order to guide the self-perfection of the nanostructures before solidification, as shown in Fig. 2.25c for sub-100 nm silicon lines on SOI. The possibility of post-process enhancement of the LER of nanostructures is an option to meet one of the most difficult requirements for IC manufacturing (LER $<3 \mathrm{~nm}$ ).
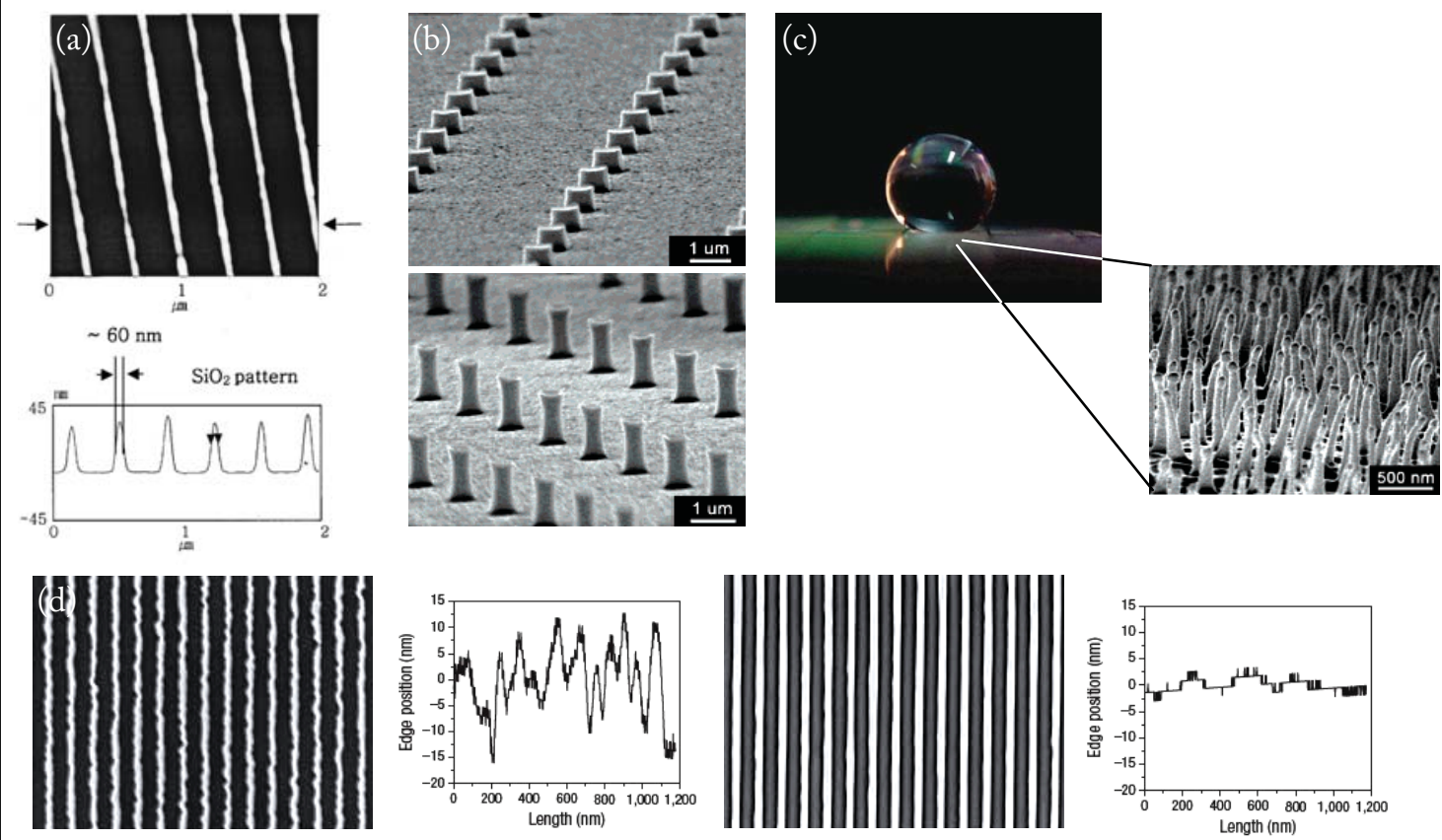

Figure 2.25 Several examples of nanostructures by contact molding: (a) high-resolution periodic lines in $\mathrm{SiO}_{2}$ by soft molding and reactive ion etching, ${ }^{[210 b]}$ (b) SEM images illustrating from top to bottom the effect of nanodrawing on the feature dimensions of PMMA nanohairs, ${ }^{[212]}$ and (c) optical image of a water droplet on a very hydrophobic surface consisting of long PMMA nanohairs (see SEM image) on 3M tape. ${ }^{[212]}$ (d) SPEL in action: $70 \mathrm{~nm}$-wide silicon lines on SOI, before (top image) and after open-SPEL by application of a single laser pulse of $545 \mathrm{~mJ} \cdot \mathrm{cm}^{-2}$ and the corresponding edge profiles illustrating a reduction of the $3 \sigma$-LER from 19.5 to $3.6 \mathrm{~nm} \cdot{ }^{[213]}$

\subsubsection{Summary}

The current set of techniques in this section is capable of replicating the surfacerelief features of hard masters into a range of materials with high accuracy, in part by the recent developments of novel stamp materials that comply with the specific tasks for soft lithography at the sub-100 $\mathrm{nm}$ scale. Unconventional techniques offer immediate advantages in the fabrication of structures that are difficult to achieve by conventional photolithography, including patterning on non-planar surfaces, fabrication of $3 \mathrm{D}$ structures, structuring materials other than the resist, and 
modification of surfaces. Especially nanoimprint lithography has the potential to become of prime importance in future IC manufacturing.

\subsection{Conclusions}

Starting from the pioneering work of Whitesides' group, the technological advancements and the number of publications over the past 15 years are illustrative for the activities of a large number of scientific groups and for the current technological status of unconventional nanofabrication in the generation and replication of nanoscale patterns. The beauty of this field lies in its diversity. The practical utility, costefficiency and versatility of unconventional techniques to structure a wide range of materials on the nanometer scale are a large stimulus for fundamental studies in materials science, chemistry, biology and physics.

The focus of this chapter was to provide an up-to-date overview of the most potential patterning principles in unconventional lithography. For the purpose of developing next-generation lithography techniques, the recent performance of nanoimprint lithography (Section 2.3.1), block copolymer lithography (Section 2.2.3.1) and dip-pen nanolithography (Section 2.2.2) in the fabrication of high-resolution patterns indicates the promise for future IC manufacturing, in spite of the fact that several important technological issues are still open for investigation. Each technique has certain characteristic advantages, and it is unlikely that a single technique will ever dominate all areas of application. Additionally, the availability and simplicity of the remaining techniques in this chapter make the fabrication of highresolution features a practical reality for many researchers in nanoscience and technology.

\subsection{References and notes}

The figures in this chapter have been reproduced from literature with permission from the publishers (Copyright @ AAAS, NPG, Wiley-VCH, ACS, APS, AIP, AVS, IOP, OSA, Elsevier, Springer).

[2] http://blogs.intel.com/technology/2007/09 (accessed at 25-02-2009)

[3] http://www.itrs.net/links/2007ITRS/home2007.htm (accessed at 25-02-2009)

[4] http://www.sia-online.org 
[5] M. D. Levenson, Solid State Technol. 1995, 57.

[6] M. Rothschild, Mater. Today 2005, 8, 18.

[7] Resolution Enhancement Techniques in Optical Lithography (Ed. A. K.-K. Wong), SPIE Optical Engineering Press, Bellingham, WA 2001.

[8] Recent review on EUV lithography: B. Wu, A. Kumar, J. Vac. Sci. Technol. B 2007, 25, 1743.

[9] For ion-beam lithography (IBL), see: (a) U. S. Tandon, Vacuum 1992, 43, 241. (b) F. Watt, A. A. Bettiol, J. A. van Kan, E. J. Teo, M. B. H. Breese, Int. J. Nanosci. 2005, 4, 269.

[10] For electron-beam lithography (EBL), see: R. S. Dhaliwal, W. A. Enichen, S. D. Golladay, M. S. Gordon, R. A. Kendall, J. E. Lieberman, H. C. Pfeiffer, D. J. Pinckney, C. F. Robinson, J. D. Rockrohr, W. Stickel, E. V. Tressler, IBM J. Res. Dev. 2001, 45, 615.

[11] For direct writing, see: J. A. Lewis, G. M. Gratson, Mater. Today 2004, 7, 32.

[12] For scanning-probe lithography (SPL), see: (a) S. Krämer, R. R. Fuierer, C. B. Gorman, Chem. Rev. 2003, 103, 4367. (b) A. A. Tseng, A. Notargiacomo, T. P. Chen, J. Vac. Sci. Technol. B 2005, 23, 877.

[13] Recent reviews on imprint lithography: (a) L. J. Guo, Adv. Mater. 2007, 19, 495. (b) L. J. Guo, J. Phys. D: Appl. Phys. 2004, 37, R123.

[14] General reviews on (unconventional) nanofabrication: (a) Y. Xia, J. A. Rogers, K. E. Paul, G. M. Whitesides, Chem. Rev. 1999, 99, 1823. (b) B. D. Gates, Q. Xu, M. Stewart, D. Ryan, C. G. Willson, G. M. Whitesides, Chem. Rev. 2005, 105, 1171.

[15] T. Blätter, C. Huwiler, M. Ochsner, B. Städler, H. Solak, J. Vörös. H. M. Grandin, J. Nanosci. Nanotechology 2006, 6, 2237.

[16] F. Watt, M. B. H. Breese, A. A. Bettiol, J. A. van Kan, Mater. Today 2007, 10, 20.

[17] C. N. LaFratta, J. T. Fourkas, T. Baldacchini, R. A. Farrer, Angew. Chem. Int. Ed. 2007, 46, 6238 .

[18] (a) J. A. Rogers, K. E. Paul, R. J. Jackman, G. M. Whitesides, Appl. Phys. Lett. 1997, 70, 2658. (b) J. A. Rogers, K. E. Paul, R. J. Jackman, G. M. Whitesides, J. Vac. Sci. Technol. B 1998, 16, 59. (c) T. W. Odom, V. R. Thalladi, J. C. Love, G. M. Whitesides, J. Am. Chem. Soc. 2002, 124, 12112.

[19] Y. Yin, B. Gates, Y. Xia, Adv. Mater. 2000, 12, 1426.

[20] H. Schmid, H. Biebuyck, B. Michel, O. J. F. Martin, N. B. Piller, J. Vac. Sci. Technol. B 1998, 16,3422 .

[21] W. Kern, C. A. Deckert, in Thin Film Processes (Eds.: J. L. Vossen, W. Kern), Academic Press, Boston, MA 1978, p. 401.

[22] J. C. Love, K. E. Paul, G. M. Whitesides, Adv. Mater. 2001, 13, 604.

[23] A. Bietsch, B. Michel, Appl. Phys. Lett. 2002, 80, 3346.

[24] (a) Y.-K. Choi, J. S. Lee, J. Zhu, G. A. Somorjai, L. P. Lee, J. Bokor, J. Vac. Sci. Technol. B 2003, 21, 2951. (b) X.-M. Yan, S. Kwon, A. M. Contreras, J. Bokor, G. A. Somorjai, 
Nano Lett. 2005, 5, 745. (c) S. Kwon, X. Yan, A. M. Contreras, J. A. Liddle, G. A. Somorjai, J. Bokor, Nano Lett. 2005, 5, 2557.

[25] K.-H. Chung, W. Y. Choi, S.-K. Sung, D. H. Kim, J. D. Lee, B.-G. Park, J. Vac. Sci. Technol. B 2003, 21, 1491.

[26] J. Haneveld, E. Berenschot, P. A. Maury, H. V. Jansen, J. Micromech. Microeng. 2006, 16, S24.

[27] X. Liang, K. J. Morton, R. H. Austin, S. Y. Chou, Nano Lett. 2007, 7, 3774.

[28] H.-W. Ra, K.-S. Choi, J.-H. Kim, Y.-B. Hahn, Y.-H. Im, Small 2008, 4, 1105.

[29] (a) K. Y. Suh, Y. S. Kim, H. H. Lee, Adv. Mater. 2001, 13, 1386. (b) K. Y. Suh, H. H. Lee, Adv. Funct. Mater. 2002, 12, 405.

[30] Y. Xia, G. M. Whitesides, Angerw. Chem. Int. Ed. 1998, 37, 550.

[31] K. Y. Suh, P. Kim, H. H. Lee, Appl. Phys. Lett. 2004, 85, 4019.

[32] (a) K. Y. Suh, P. J. Yoo, H. H. Lee, Macromolecules 2002, 35, 4414. (b) K. Y. Suh, S. Chu, H. H. Lee, J. Micromech. Microeng. 2004, 14, 1185.

[33] (a) J. M. McLellan, M. Geissler, Y. Xia, J. Am. Chem. Soc. 2004, 126, 10830. (b) M. Geissler, J. M. McLellan, Y. Xia, Nano Lett. 2005, 5, 31.

[34] (a) J. C. Hulteen, R. P. van Duyne, J. Vac. Sci. Technol. A 1995, 13, 1553. (b) C. L. Haynes, R. P. van Duyne, J. Phys. Chem. B 2001, 105, 5599.

[35] H. A. Biebuyck, G. M. Whitesides, Langmuir 1994, 10, 4581.

[36] (a) E. Delamarche, H. Schmid, A. Bietsch, N. B. Larsen, H. Rothuizen, B. Michel, H. Biebuyck, J. Phys. Chem. B 1998, 102, 3324. b) L. Libiuouelle, A. Bietsch, H. Schmid, B. Michel, E. Delamarche, Langmuir 1999, 15, 300.

[37] (a) M. Liebau, J. Huskens, D. N. Reinhoudt, Adv. Funct. Mater. 2001, 11, 147. (b) A. Perl, M. Péter, B. J. Ravoo, D. N. Reinhoudt, J. Huskens, Langmuir 2006, 22, 7568.

[38] O. Cherniavkaya, A. Adzic, C. Knotson, B. J. Gross, L. Zang, R. Liu, D. M. Adams, Langmuir 2002, 18, 7029.

[39] M. Geissler, J. M. McLellan, J. Chen, Y. Xia, Angerw. Chem. Int. Ed. 2005, 44, 3596.

[40] M. Geissler, P. Chalsani, N. S. Cameron, T. Veres, Small 2006, 2, 760.

[41] M. T. Zin, H.-L. Yip, N.-Y. Wong, H. Ma, A. K.-Y. Jen, Langmuir 2006, 22, 6346.

[42] (a) J. Aizenberg, A. J. Black, G. M. Whitesides, Nature 1998, 394, 868. (b) A. J. Black, K. E. Paul, J. Aizenberg, G. M. Whitesides, J. Am. Chem. Soc. 1999, 121, 8356.

[43] X.-M Zhao, J. L. Wilbur, G. M. Whitesides, Langmuir 1996, 12, 3257.

[44] M. Geissler, H. Schmid, A. Bietsch, B. Michel, E. Delamarche, Langmuir 2002, 18, 2374.

[45] H. Yang, J. C. Love, F. Arias, G. M. Whitesides, Chem. Mater. 2002, 14, 1385.

[46] K. E. Paul, T. L. Breen, J. Aizenberg, G. M. Whitesides, Appl. Phys. Lett. 1998, 73, 2893.

[47] J. L. Wilbur, E. Kim, Y. Xia, G. M. Whitesides, Adv. Mater. 1995, 7, 649.

[48] H.-W Li, B. V. O. Muir, G. Fichet, W. T. S. Huck, Langmuir 2003, 19, 1963. 
[49] Z. Yu, S. Y. Chou, Nano Lett. 2004, 4, 341.

[50] (a) Y. Xia, E. Kim, X.-M. Zhao, J. A. Rogers, M. Prentiss, G. M. Whitesides, Science 1996, 273, 347. (b) Y. Xia, J. J. McClelland, R. Gupta, D. Qin, X.-M. Zhao, L. L. Sohn, R. J. Celotta, G. M. Whitesides, Adv. Mater. 1997, 9, 147.

[51] Q. Xu, B. Gates, G. M. Whitesides, J. Am. Chem. Soc. 2004, 126, 1332.

[52] N.A. Melosh, A. Boukai, F. Diana, B. Gerardot, A. Badolato, P. M. Petroff, J. R. Heath, Science 2003, 300, 112.

[53] (a) M. D. Austin, H. Ge, W. Wu, M. Li, Z. Yu, D. Wasserman, S. A. Lyon, S. Y. Chou, Appl. Phys. Lett. 2004, 84, 5299. (b) G.-Y. Jung, E. Johnston-Halperin, W. Wu, Z. Yu, S.-Y. Wang, W. M. Tong, Z. Li, J. E. Green, B. A. Sheriff, A. Boukai, Y. Bunimovich, J. R. Heath, R. S. Williams, Nano Lett. 2006, 6, 351. (c) D. Wang, Y. Bunimovich, A. Boukai, J. R. Heath, Small 2007, 3, 2043.

[54] (a) L. Xu, S. C. Vemula, M. Jain, S. K. Nam, V. M. Donnelly, D. J. Economou, P. Ruchhoeft, Nano Lett. 2005, 5, 2563. (b) L. Xu, A. Nasrullah, Z. Chen, M. Jain, P. Ruchhoeft, D. J. Economou, V. M. Donnelly, Appl. Phys. Lett. 2008, 92, 013124.

[55] P. Vettiger, M. Despont, U. Drechsler, U. Dürig, W. Häberle, M. I. Lutwyche, H. E. Rothuizen, R. Stutz, R. Widmer, G. K. Binnig, IBM J. Res. Dev. 2000, 44, 323.

[56] (a) R. D. Piner, J. Zhu, F. Xu, S. H. Hong, C. A. Mirkin, Science 1999, 283, 661.

(b) S. H. Hong, C. A. Mirkin, Science 2000, 288, 1808.

[57] D. S. Ginger, H. Zhang, C. A. Mirkin, Angew. Chem. Int. Ed. 2004, 43, 30.

[58] (a) H. Zhang, S.-W. Chung, C. A. Mirkin, Nano Lett. 2003, 3, 43. (b) A. Ivanisevic, K. V. McCumber, C. A. Mirkin, J. Am. Chem. Soc. 2002, 124, 11997.

[59] B. W. Maynor, S. F. Filocamo, M. W. Grinstaff, J. Liu, J. Am. Chem. Soc. 2002, 124, 522. (b) J. H. Lim, C. A. Mirkin, Adv. Mater. 2002, 14, 1474. (c) A. Noy, A. E. Miller, J. E. Klare, B. L. Weeks, B. W. Woods, J. J. DeYoreo, Nano Lett. 2002, 2, 109.

[60] (a) L. M. Demers, D. S. Ginger, S. J. Park, Z. Li, S.W. Chung, C. A. Mirkin, Science 2002, 296, 1836. (b) D. L. Wilson, R. Martin, S. Hong, M. Cronin-Golomb, C. A. Mirkin, D. L. Kaplan, Proc. Natl. Acad. Sci. USA 2001, 98, 13660.

[61] J. C. Garno, Y. Yang, N. A. Amro, S. Cruchon-Dupeyrat, S. Chen, G.-Y. Liu, Nano Lett. 2003, 3, 389.

[62] (a) Y. Li, B. W. Maynor, J. Liu, J. Am. Chem. Soc. 2001, 123, 2105. (b) B.W. Maynor, Y. Li, J. Liu, Langmuir 2001, 17, 2575.

[63] (a) B. L. Weeks, A. Noy, A. E. Miller, J. J. De Yoreo, Phys. Rev. Lett. 2002, 88, 255505.

(b) P. Manandhar, J. Jang, G. C. Schatz, M. A. Ratner, S. Hong, Phys. Rev. Lett. 2003, 90, 115505.

[64] S. Rozhok, R. Piner, C. A. Mirkin, J. Phys. Chem. 2003, 107, 751.

[65] J. Jang, S. Hong, G. C. Schatz, M. A. Ratner, J. Chem. Phys. 2001, 115, 2721. 
[66] D. A. Weinberger, S. Hong, C. A. Mirkin, B. W. Wessels, T. B. Higgins, Adv. Mater. 2000, 12,1600 .

[67] J.-W. Jang, D. Maspoch, T. Fujigaya, C. A. Mirkin, Small 2007, 3, 600.

[68] (a) K. Salaita, S. W. Lee, X. Wang, L. Huang, T. M. Dellinger, C. Liu, C. A. Mirkin, Small 2005, 1, 940. (b) H. Zhang, N. A. Amro, S. Disawal, R. Elghanian, R. Shile, J. Fragala, Small 2007, 3, 81 .

[69] K. Salaita, Y. Wang, J. Fragala, R. A. Vega, C. Liu, C. A. Mirkin, Angew. Chem. Int. Ed. 2006, 45, 7220 .

[70] M. Zhang, D. Bullen, S.-W. Chung, S. Hong, K. S. Ryu, Z. Fan, C. A. Mirkin, C. Liu, Nanotechology 2002, 13, 212.

[71] (a) X. Wang, K. S. Ryu, D. A. Bullen, J. Zou, H. Zhang, C. A. Mirkin, C. Liu, Langmuir 2003, 19, 8951. (b) H. Zhang, R. Elghanian, N. A. Amro, S. Disawal, R. Eby, Nano Lett. 2004, 4, 1649.

[72] B. Rosner, T. Duenas, D. Banerjee, R. Shile, N. Amro, J. Rendlen, Smart Mater. Struct. 2006, 15, S124.

[73] http://www.nanoink.net

[74] F. Huo, Z. Zheng, G. Zheng, L. R. Giam, H. Zhang, C. A. Mirkin, Science 2008, 321, 1658.

[75] J. M. Lehn, Angew. Chem. Int. Ed. 1990, 29, 1304.

[76] F. S. Bates, G. H. Fredrickson, Annu. Rev. Phys. Chem. 1990, 41, 525.

[77] F. S. Bates, Science 1991, 251, 898.

[78] I. W. Hamley, The Physics of Block Copolymers, Oxford University Press, NY 1998.

[79] Recent reviews on block copolymer lithography: (a) M. P. Stoykovich, P. F. Nealy, Mater. Today 2006, 9, 20. (b) M. Li, C. K. Ober, Mater. Today 2006, 9, 30. (c) S. Krishnamoorthy, C. Hinderling, H. Heinzelmann, Mater. Today 2006, 9, 40.

[80] (a) M. J. Fasolka, D. J. Harris, A. M. Mayes, M. Yoon, S. G. J. Mochrie, Appl. Phys. Lett. 1997, 79, 3018. (b) J. Y. Cheng, C. A. Ross, E. L. Thomas, H. I. Smith, G. J. Vancso, Appl. Phys. Lett. 2002, 81, 3657.

[81] (a) E. Huang, L. Rockford, T. P. Russell, C. J. Hawker, Nature 1998, 395, 757. (b) S. O. Kim, H. H. Solak, M. P. Stoykovich, N. J. Ferrier, J. J. de Pablo, P. F. Nealey, Nature 2003, 424, 411.

[82] T. L. Morkved, M. Lu, A. M. Urbas, E. E. Ehrichs, H. M. Jaeger, P. Mansky, T. P. Russell, Science 1996, 273, 931.

[83] (a) J. Y. Cheng, C.A. Ross, E.L. Thomas, H.I. Smith, G.J. Vancso, Adv. Mater. 2003, 15, 1599. (b) J. Y. Cheng, F. Zhang, H. I. Smith, G. J. Vancso, C. A. Ross, Adv. Mater. 2006, 18, 597. 
[84] (a) P. Du, Li, K. Douki, X. Li, C. B. W. Garcia, A. Jain, D.-M. Smilgies, L. J. Fetters, S. M. Gruner, U. Wiesner, C. K. Ober, Adv. Mater. 2004, 16, 953. (b) M. Li, K. Douki, K. Goto, X. Li, C. Coenjarts, D. M. Smilgies, C. K. Ober, Chem. Mater. 2004, 16, 3800.

[85] M. Park, C. Harrison, P. M. Chaikin, R. A. Register, D. H. Adamson, Science 1997, 276, 1401.

[86] V. Z.-H. Chan, J. Hoffman, V. Y. Lee, H. Latrou, A. Avgeropoulos, N. Hadjichristidis, R. D. Miller, E. L. Thomas, Science 1999, 286, 1716.

[87] (a) J. Y. Cheng, C. A. Ross, V. Z.-H. Chan, E. L. Thomas, R. G. H. Lammertink, G. J. Vancso, Adv. Mater. 2001, 13, 1174. (b) R. G. H. Lammertink, M. A. Hempenius, V. Z.-H. Chan, E. L. Thomas, G. J. Vancso, Chem. Mater. 2001, 13, 429.

[88] K. Fukukawa, L. Zhu, P. Gopalan, M. Ueda, S. Yang, Macromolecules 2005, 38, 263.

[89] D. H. Kim, Z. Lin, H.-C. Kim, U. Jeong, T. P. Russell, Adv. Mater. 2003, 15, 811.

[90] C. T. Black, K. W. Guarini, K. R. Milkove, S. M. Baker, T. P. Russell, M. T. Tuominen, Appl. Phys. Lett. 2001, 79, 409.

[91] R. R. Li, P. D. Dapkus, M. E. thompson, W. G. Jeong, C. Harrison, P. M. Chaikin, R. A. Register, D. H. Adamson, App. Phys. Lett. 2000, 76, 1689.

[92] I. A. Ansari, I. W. Hamley, J. Mater. Chem. 2003, 13, 2412.

[93] (a) T. Thurn-Albrecht, J. Schotter, G. A. Kästle, N. Emley, T. Shibauchi, L. Krusin-Elbaum, K. Guarini, C. T. Black, M. T. Tuominen, T. P. Russell, Science 2000, 290, 2126. (b) W. A. Lopes, H. M. Jaeger, Nature 2001, 414, 735.

[94] J. Q. Lu, T. E. Kopley, N. Moll, D. Roitman, D. Chamberlin, Q. Fu, J. Liu, T. P. Russell, D. A. Rider, I. Manners, M. A. Winnink, Chem. Mater. 2005, 17, 2227.

[95] H.-C. Kim, X. Jia, C. M. Stafford, D. H. Kim, T. J. McCarthy, M. Tuominen, C. J. Hawker, T. P. Russell, Adv. Mater. 2001, 13, 795.

[96] (a) U. Jeong, H.-C. Kim, R. L. Rodriquez, I. Y. Tsai, C. M. Stafford, J. K. Kim, C. J. Hawker, T. P. Russell, Adv. Mater. 2002, 14, 274. (b) K. Shin, K. A. Leach, J. T. Goldbach, D. H. Kim, J. Y. Jho, M. Tuominen, C. J. Hawker, T. P. Russell, Nano Lett. 2002, 2, 933.

[97] Y. Fink, A. M. Urbas, M. G. Bawendi, J. D. Joannopoulos, E. L. Thomas, J. Lightwave Technol. 1999, 17, 1963.

[98] A. C. Edrington, A. M. Urbas, P. DeRege, C. X. Chen, T. M. Swager, N. Hadjichristidis, M. Xenidou, L. J. Fetters, J. D. Joannopoulos, Y. Fink, E. L. Thomas, Adv. Mater. 2001, 13, 421.

[99] (a) A. M. Urbas, R. Sharp, Y. Fink, E. L. Thomas, M. Xenidou, L. J. Fetters, Adv. Mater. 2000, 12, 812. (b) A. M. Urbas, M. Maldovan, P. DeRege, E. L. Thomas, Adv. Mater. 2002, 14,1850 . 
[100] Y. Lin, A. Böker, J. He, K. Sill, H. Xiang, C. Abetz, X. Li, J. Wang, T. Emrick, S. Long, Q. Wang, A. Balazs, T. P. Russell, Nature 2005, 434, 55.

[101] A. M. Urbas, Y. Fink, and E. L. Thomas, Macromolecules 1999, 32, 4748.

[102] A. N. Semenov, Macromolecules 1993, 26, 6617.

[103] M. P. Stoykovich, M. Müller, S. O. Kim, H. H. Solak, E. W. Edwards, J. J. de Pablo, P. F. Nealey, Science 2005, 308, 1442.

[104] (a) K. Y. Suh, H. H. Lee, Adv. Mater. 2002, 14, 346. (b) K. Y. Suh, H. H. Lee, J. Micromech. Microeng. 2005, 15, 400.

[105] A. M. Higgins, R. A. L. Jones, Nature 2000, 404, 476.

[106] A. Birner, R. B. Wehrspohn, U. M. Gösele, K. Busch, Adv. Mater. 2001, 13, 377.

[107] Y. Xia, Y. Yin, Y. Lu, J. McLellan, Adv. Funct. Mater. 2003, 13, 907.

[108] P. T. Hammond, Adv. Mater. 2004, 16, 1271.

[109] Y. Xia, B. Gates, Y. Yin, Y. Lu, Adv. Mater. 2000, 12, 693.

[110] A. van Blaaderen, R. Ruel, P. Wiltzius, Nature 1997, 385, 321.

[111] Y. A. Vlasov, X.-Z. Bo, J. C. Sturm, D. J. Norris, Nature 2001, 414, 289.

[112] C. Bae, H. Shin, J. Moon, M. M. Sung, Chem. Mater. 2006, 18, 1085.

[113] C. L. Cheung, R. J. Nikolić, C. E. Reinhardt, T. F. Wang, Nanotechology 2006, 17, 1339.

[114] B. J. Y. Tan, C. H. Sow, T. S. Koh, K. C. Chin, A. T. S. Wee, C. K. Ong, J. Phys. Chem. B $2005,109,11100$.

[115] L. Schubert, P. Werner, U. Gösele, Nano Lett. 2005, 5, 2524.

[116] K. H. Park, S. Lee, K. H. Koh, R. Lacerda, K. B. K. Teo, W. I. Milne, J. Appl. Phys. 2005, 97, 024311.

[117] D. F. Liu, Y. J. Xiang, Q. Liao, J. P. Zhang, X. C. Wu, Z. X. Zhang, L. F. Liu, W. J. Ma, J. Shen, W. Y. Zhou, S. S. Xie, Nanotechology 2007, 18, 405303.

[118] (a) C.-W. Kuo, J.-Y. Shiu, Y.-H. Cho, P. Chen, Adv. Mater. 2003, 15, 1065. (b) C.-W. Kuo, J.-Y. Shiu, P. Chen, G. A. Somorjai, J. Phys. Chem. B 2003, 107, 9950.

[119] S. Han, Z. Hao, J. Wang, Y. Luo, J. Vac. Sci. Technol. B 2005, 23, 1585.

[120] K. Peng, M. Zhang. A. Lu, N.-B. Wong, R. Zhang, S.-T. Lee, Appl. Phys. Lett. 2007, 90, 163123.

[121] (a) A. Kosiorek, W. Kandulski, P. Chudzinski, K. Kempa, M. Giersig, Nano Lett. 2004, 4, 1359. (b) A. Kosiorek, W. Kandulski, H. Glaczynska, M. Giersig, Small 2005, 1, 439.

[122] Y. B. Zheng, S. J. Wang, A. C. H. Huan, Y. H. Wang, J. Appl. Phys. 2006, 99, 034308.

[123] P. A. Malachesky, in Encyclopedia of Electrochemistry of the Elements (Ed. A. J. Bard), Vol. 4, Marcel Dekker, NY 1973, p. 63.

[124] R. C. Furneaux, W. R. Rigby, A. P. Davidson, Nature 1989, 337, 147.

[125] Y. Lei, W. Cai, G. Wilde, Prog. Mater. Sci. 2007, 52, 465. 
[126] O. Jessensky, F. Muller, U. Gösele, J. Appl. Phys. 1998, 72, 1173.

[127] (a) H. Masuda, M. Satoh, Jpn. J. Appl. Phys. 1996, 35, L126. (b) H. Masuda, H. Yamada, M. Satoh, H. Asoh, M. Nakao, T. Tamamura, Appl. Phys. Lett. 1997, 71, 2770.

[128] (a) K. Nielsch, F. Muller, A. P. Li, U. Gösele, Adv. Mater. 2000, 12, 582. (b) H. Q. Cao, Z. Xu, H. Sang, D. Sheng, C. Y. Tie, Adv. Mater. 2001, 13, 121. (c) C. Mu, Y. X. Yn, R. M. Wang, K. Wu, D. S. Xu, G. L. Guo, Adv. Mater. 2004, 16, 1550.

[129] (a) D. Routkevitch, T. Bigioni, M. Moskovits, J. M. Xu, J. Phys. Chem. 1996, 100, 14037. (b) Y. Lei, L. D. Zhang, G. W. Meng, G. H. Li, X. Y. Zhang, C. H. Liang, W. Chen, S. X. Wang, Appl. Phys. Lett. 2001, 78, 1125.

[130] (a) G. L. Che, B. B. Lakshmi, E. R. Fisher, C. R. Martin, Nature 1998, 393, 346. (b) J. Li, C. Papadopoulos, J. M. Xu, Appl. Phys. Lett. 1999, 402, 253.

[131] (a) Masuda, K. Fukuda, Science 1995, 268, 1466. (b) T. Yanagishita, K. Nishio, H. Masuda, Adv. Mater. 2005, 17, 2241.

[132] (a) W. Lee, J.-K. Lee, Adv. Mater. 2002, 14, 1187. (b) C. Goh, K. M. Coakley, M. D. McGehee, Nano Lett. 2005, 5, 1545.

[133] P.-S. Lee, O.-J. Lee, S.-K. Hwang, S.-H. Jung, S. E. Jee, K.-H. Lee, Chem. Mater. 2005, 17, 6181.

[134] (a) C.-S. Toh, B. M. Kayes, E. J. Nemanick, N. S. Lewis, Nano Lett. 2004, 4, 767. (b) M. Tian, S. Xu, J. Wang, N. Kumar, E. Wertz, Q. Li, P. M. Campbell, M. H. W. Chan, T. E. Mallouk, Nano Lett. 2005, 5, 697.

[135] (a) X. Y. Mei, D. Kim, H. E. Ruda, Q. X. Guo, Appl. Phys. Lett. 2002, 81, 361. (b) K. Liu, J. Nogués, C. Leighton, H. Masuda, K. Nishio, I. V. Roshchin, I. K. Schuller, Appl. Phys. Lett. 2002, 81, 4434. (c) Y. Lei, W. K. Chim, H. P. Sun, G. Wilde, Appl. Phys. Lett. 2005, 86, 103106.

[136] (a) D. Crouse, Y.-H. Lo, A. E. Miller, M. Crouse, Appl. Phys. Lett. 2000, 76, 49.

(b) Y. Kanamori, K. Hane, H. Sai, H. Yugami, Appl. Phys. Lett. 2001, 78, 142.

(c) N. Matsuura, T. W. Simpson, I. V. Mitchell, X.-Y. Mei, P. Morales, H. E. Ruda, Appl. Phys. Lett. 2002, 81, 4826.

[137] (a) T. Iwasaki, T. Motoi, T. Den, Appl. Phys. Lett. 1999, 75, 2044. (b) G. Q. Ding, W. Z. Shen, M. J. Zheng, D. H. Fan, Appl. Phys. Lett. 2006, 88, 103106.

[138] (a) A.-P. Li, F. Müller, A. Birner, K. Nielsch, U. Gösele, Adv. Mater. 1999, 11, 483. (b) N.-W. Liu, A. Datta, C.-Y. Liu, C.-Y. Peng, H.-H. Wang, Y.-L. Wang, Adv. Mater. 2005, 17, 222.

[139] M. Nakao, S. Oku, H. Tanaka, Y. Shibata, A. Yokoo, T. Tamamura, H. Masuda, Opt. Quant. Electron. 2002, 34, 183.

[140] J. Luo, Z. Huang, Y. Zhao, L. Zhang, J. Zhu, Adv. Mater. 2004, 16, 1512.

[141] S. Y. Chou, P. R. Krauss, P. J. Renstrom, Science 1996, 272, 85. 
patterning principles

[142] S. Y. Chou, P. R. Krauss, W. Zhang, L. Guo, L. Zhang, J. Vac. Sci. Technol. B 1997, 15, 2897.

[143] Y. Nakada, K. Nimomiya, Y. Takaki, Jpn. J. Appl. Phys. 2006, 45, L1241.

[144] (a) Q. Xia, C. Keimel, H. Ge, Z. Yu, W. Wu, S. Y. Chou, Appl. Phys. Lett. 2003, 83, 4417. (b) S. Y. Chou, C. Keimel, J. Gu, Nature 2002, 417, 835.

[145] (a) Y. Igaku, S. Matsui, H. Ishigaki, J. Fujita, M. Ishida, Y. Ochiai, H. Namatsu, M. Komuro, H. Hiroshima, Jpn. J. Appl. Phys. 2002, 41, 4198. (b) S. Matsui, Y. Igaku, H. Ishigaki, J. Fujita, M. Ishida, Y. Ochiai, H. Namatsu, M. Komuro, J. Vac. Sci. Technol. B 2003, 21, 688. (c) M. Kawamori, K. Nakamatsu, Y. Haruyama, S. Matsui, Jpn. J. Appl. Phys. 2006, 45, 8994.

[146] (a) D.-Y. Khang, H. H. Lee, Appl. Phys. Lett. 2000, 76, 870. (b) D.-Y. Chang, H. Yoon, H. H. Lee, Adv. Mater. 2001, 13, 749.

[147] X. Cheng, L. J. Guo, P.-F. Fu, Adv. Mater. 2005, 17, 1419.

[148] H. Gao, H. Tan, W. Zhang, K. Morton, S. Y. Chou, Nano Lett. 2006, 6, 2438.

[149] Y. Guo, G. Liu, Y. Xiong, Y. Tian, J. Micromech. Microeng. 2007, 17, 9.

[150] (a) H.-J. Lee, H. W. Ro, C. L. Soles, R. L. Jones, E. K. Lin, W. Wu, D. R. Hines, J. Vac. Sci. Technol. B 2005, 23, 3023. (b) H.-J. Lee, C. L. Soles, H. W. Ro, R. L. Jones, E. K. Lin, W. Wu, D. R. Hines, Appl. Phys. Lett. 2005, 87, 263111.

[151] N. Li, W. Wu, S. Y. Chou, Nano Lett. 2006, 6, 2626.

[152] http://www.obducat.com (Intermediate Polymer Stamp (IPS) ${ }^{\circledR}$, including European Patent: EP1731962, accessed at 25-02-2009)

[153] (a) Q. Xia, Z. Yu, H. Gao, S. Y. Chou, Appl. Phys. Lett. 2006, 89, 073107. (b) Z. Yu, H. Gao, S. Y. Chou, Nanotechnology 2007, 18, 065304.

[154] (a) H. Schultz, M. Wissen, N. Bogdanski, H.-C. Scheer, K. Mattes, C. Friedrich, Microelectron. Eng. 2006, 83, 259. (b) H.-C. Scheer, N. Bogdanski, M. Wissen, S. Möllenbeck, J. Vac. Sci. Technol. B 2007, 25, 2392. (c) H.-C. Scheer, N. Bogdanski, M. Wissen, Jpn. J. Appl. Phys. 2005, 44, 5609.

[155] C.-H. Hsueh, S. Lee, H.-Y. Lin, L.-S. Chen, W.-H. Wang, Mat. Sci. Eng. A 2006, 433, 316.

[156] Y. Ding, H. W. Ro, J. F. Douglas, R. L. Jones, D. R. Hine, A. Karim, C. L. Soles, Adv. Mater. 2007, 19, 1377.

[157] (a) R. W. Jaszewski, H. Schift, B. Schnyder, A. Schneuwly, P. Gröning, Appl. Surf. Sci. 1999, 143, 301. (b) K. Nakamatsu, N. Yamada, K. Kanda, Y. Haruyama, S. Matsui, Jpn. J. Appl. Phys. 2006, 45, L954. (c) N. Yamada, K. Nakamatsu, K. Kanda, Y. Haruyama, S. Matsui, Jpn. J. Appl. Phys. 2007, 46, 6373.

[158] (a) G.-Y. Jung, Z. Li, W. Wu, Y. Chen, D. L. Olynick, S.-Y. Wang, W. M. Tong, R. S. Williams, Langmuir 2005, 21, 1158. (b) C.-F. Wang, S.-F. Chiou, F.-H. Ko, J.-K. Chen, C.-T. Chou, C.-F. Huang, S.-W. Kuo, F.-C. Chang, Langmuir 2007, 23, 5868. 
[159] (a) D.-Y. Khang, H. Kang, T. Kim, H. H. Lee, Nano Lett. 2004, 4, 633. (b) D.-Y. Khang, H. H. Lee, Langmuir 2004, 20, 2445.

[160] D. G. Choi, J. H. Jeong, Y. S. Sim, E. S. Lee, W. S. Kim, B. S. Bae, Langmuir 2005, 21, 9390.

[161] H. D. Rowland, A. C. Sun, P. R. Schunk, W. P. King, J. Micromech. Microeng. 2005, 15, 2414.

[162] Y. Chen, J. Tao, X. Zhao, Z. Cui, J. Microlithogr. Microfabr. Microsyst. 2006, 5, 011002.

[163] M. Li, L. Chen, W. Zhang, S. Y. Chou, Nanotechnology 2003, 14, 33.

[164] D. Suh, S.-J. Choi, H. H. Lee, Adv. Mater. 2005, 17, 1554.

[165] J. Haisma, M. Verheijen, K. van den Heuvel, J. van den Berg, J. Vac. Sci. Technol. B 1996, 14, 4124.

[166] (a) H.-C. Scheer, N. Bogdanski, M. Wissen, S. Möllenbeck, Microelectron. Eng. 2008, 85, 890. (b) H.-C. Scheer, N. Bogdanski, M. Wissen, T. Konishi, Y. Hirai, J. Vac. Sci. Technol. B 2005, 23, 2963. (c) H.-C. Scheer, H. Schultz, Microelectron. Eng. 2001, 56, 311.

[167] C. Pina-Hernandez, J.-S. Kim, L. J. Guo, P.-F. Fu, Adv. Mater. 2007, 19, 1222.

[168] P. Choi, P.-F. Fu, L. J. Guo, Adv. Funct. Mater. 2007, 17, 65.

[169] X.-M. Zhao, Y. Xia, G. M. Whitesides, Adv. Mater. 1996, 8, 837.

[170] (a) L.-R. Bao, X. Cheng, X. D. Huang, L. J. Guo, S. W. Pang, A. F. Yee, J. Vac. Sci. Technol. B 2002, 20, 2881. (b) H. Park, H. Li, X. Cheng, J. Vac. Sci. Technol. B 2007, 25, 2325.

[171] (a) X. D. Huang, L.-R. Bao, X. Cheng, L. J. Guo, S. W. Pang, A. F. Yee, J. Vac. Sci. Technol. B 2002, 20, 2872. (b) H. Ooe, M. Morimatsu, T. Yoshikawa, H. Kawata, Y. Hirai, J. Vac. Sci. Technol. B 2005, 23, 375.

[172] S. Ogawa, M. Imada, S. Yoshimoto, M. Okano, S. Noda, Science 2004, 305, 227.

[173] M. C. McAlpine, R. S. Friedman, C. M. Lieber, Nano Lett. 2003, 3, 443.

[174] D. Li, L. J. Guo, Appl. Phys. Lett. 2006, 88, 063513.

[175] (a) L. Guo, P. R. Krauss, S. Y. Chou, Appl. Phys. Lett. 1997, 71, 1881. (b) W. Zhang, S. Y. Chou, Appl. Phys. Lett. 2003, 83, 1632.

[176] P. Del Carro, A. Camposeo, R. Stabile, E. Mele, L. Persano, R. Cingolani, D. Pisignano, Appl. Phys. Lett. 2006, 89, 201105.

[177] (a) M. Okinaka, S. Inoue, K. Tsukagoshi, Y. Aoyagi, J. Vac. Sci. Technol. B 2006, 24, 271.

(b) M. Okinaka, S. Inoue, K. Tsukagoshi, Y. Aoyagi, J. Vac. Sci. Technol. B 2007, $25,899$.

[178] S. Jeon, J.-W. Kang, H.-D. Park, J.-J. Kim, J. R. Youn, J. Shim, J. Jeong, D.-G. Choi, K.-D. Kim, A. O. Altun, S.-H. Kim, Y.-H. Lee, Appl. Phys. Lett. 2008, 92, 223307.

[179] J. J. Wang, L. Chen, X. Liu, P. Sciortino, F. Liu, F. Walters, X. Deng, Appl. Phys. Lett. 2006, 89, 141105.

[180] G. M. McClelland, M. W. Hart, C. T. Rettner, M. E. Best, K. R. Carter, B. D. Terris, Appl. Phys. Lett. 2002, 81, 1483. 
patterning principles

[181] L. J. Guo, X. Cheng, C.-F. Chou, Nano Lett. 2004, 4, 69.

[182] (a) J. D. Hoff, L.-J. Cheng, E. Meyhöfer, L. J. Guo, A. J. Hunt, Nano Lett. 2004, 4, 853.

(b) D. Falconet, D. Pasqui, S. Park, R. Eckert, H. Schift, J. Gobrecht, R. Barbucci, M. Textor, Nano Lett. 2004, 4, 1909.

[183] P. I. Borel, B. Bilenberg, L. H. Frandsen, T. Nielsen, J. Fage-Pedersen, A. V. Lavrinenko, J. S. Jensen, O. Sigmund, A. Kristensen, Opt. Express 2007, 15, 1261.

[184] S. J. Clarson, J. A. Semlyen, Siloxane polymers, Prentice Hall: Englewood Cliffs, NJ 1993.

[185] J. N. Lee, C. Park, G. M. Whitesides, Anal. Chem. 2003, 75, 6544.

[186] (a) E. Delamarche, C. Donzel, F. S. Kamounah, H. Wolf, M. Geissler, R. Stutz, P. SchmidtWinkel, B. Michel, H. J. Mathieu, K. Schaumburg, Langmuir 2003, 19, 8749. (b) J. Lee, M. J. Kim, H. H. Lee, Langmuir 2006, 22, 2090. (c) D. Wang, R. D. Oleschuk, J. H. Horton, Langmuir 2008, 24, 1080.

[187] (a) E. Delamarche, H. Schmid, B. Michel, H. Biebuyck, Adv. Mater. 1997, 9, 741.

(b) C. Y. Hui, A. Jagota, Y. Y. Lin, E. J. Kramer, Langmuir 2002, 18, 1394.

[188] (a) H. Schmid, B. Michel, Macromolecules 2000, 33, 3042. (b) T. W. Odom, J. C. Love, D. B. Wolfe, K. E. Paul, G. M. Whitesides, Langmuir 2002, 18, 5314. (c) K. M. Choi, J. A. Rogers, J. Am. Chem. Soc. 2003, 125, 4060.

[189] (a) P. J. Yoo, S.-J. Choi, J. H. Kim, D. Suh, S. J. Baek, T. W. Kim, H. H. Lee, Chem. Mater. 2004, 16, 5000. (b) S.-J. Choi, P. J. Yoo, S. J. Baek, T. W. Kim, H. H. Lee, J. Am. Chem. Soc. 2004, 126, 7744.

[190] J. P. Rolland, E. C. Hagberg, G. M. Denison, K. R. Carter, J. M. DeSimone, Angerw. Chem. Int. Ed. 2004, 43, 5796.

[191] J. Park, Y. S. Kim, Nano Lett. 2005, 5, 1347.

[192] G. Csucs, T. Künzler, K. Feldman, F. Robin, N. D. Spencer, Langmuir 2003, 19, 6104.

[193] K. Glasmäster, J. Gold, A.-S. Andersson, D. S. Sutherland, B. Kasemo, Langmuir 2003, 19, 5475 .

[194] (a) B. D. Gates, G. M. Whitesides, J. Am. Chem. Soc. 2003, 125, 14986. (b) F. Hua, Y. Sun, A. Gaur, M. A. Meitl, L. Bilhaut, L. Rotkina, J. Wang, P. Geil, M. Shim, J. A. Rogers, Nano Lett. 2004, 4, 2467.

[195] N. B. Larsen, H. Biebuyck, E. Delamarche, B. Michel, J. Am. Chem. Soc. 1997, 119, 3017.

[196] J. C. Love, L. A. Estroff, J. K. Kriebel, R. G. Nuzzo, G. M. Whitesides, Chem. Rev. 2005, $105,1103$.

[197] (a) Y. Xia, X.-M. Zhao, E. Kim, G. M. Whitesides, Chem. Mater. 1995, 7, 2332.

(b) N. L. Jeon, R. G. Nuzzo, Y. Xia, M. Mrksich, G. M. Whitesides, Langmuir 1995, 11, 3024. 
[198] (a) P. C. Hidber, W. Helbig, E. Kim, G. M. Whitesides, Langmuir 1996, 12, 1375. (b) A. Bernard, E. Delemarche, H. Schmid, B. Michel, H. R. Bosshard, H. Biebuyck, Langmuir 1998, 14, 2225. (c) J. Lahiri, E. Ostuni, G. M. Whitesides, Langmuir 1999, 15, 2055. (d) H. Kind, J.-M. Bonard, C. Emmenegger, L.-O. Nilsson, K. Hernadi, E. MaillardSchaller, L. Schlapbach, L. Forró, K. Kern, Adv. Mater. 1999, 11, 1285. (e) X. C. Wu, A. M. Bittner, K. Kern, Langmuir 2002, 18, 4984. (f) J. Park, P. T. Hammond, Adv. Mater. 2004, 16, 520.

[199] (a) Y. Xia, J. Tien, D. Qin, G. M. Whitesides, Langmuir 1996, 12, 4033. (b) Y. Xia, G. M. Whitesides, Langmuir 1997, 13, 2059.

[200] H. Schmid, H. Wolf, R. Allenspach, H. Reil, S. Karg, B. Michel, E. Delamarche, Adv. Funct. Mater. 2003, 13, 145.

[201] (a) Y.-L. Loo, R. L. Willet, K. W. Baldwin, J. A. Rogers, J. Am. Chem. Soc. 2002, 124, 7654.

(b) Y.-L. Loo, R. L. Willet, K. W. Baldwin, J. A. Rogers, Appl. Phys. Lett. 2002, 81, 562.

(c) Y.-L. Loo, D. V. Lang, J. A. Rogers, J. W. P. Hsu, Nano Lett. 2003, 3, 913.

[202] Z. Wang, J. Yuan, J. Zhang, R. Xing, D. Yan, Y. Han, Adv. Mater. 2003, 15, 1009.

[203] (a) L.-R. Bao, L. Tan, X. D. Huang, Y. P. Kong, L. J. Guo, S. W. Pang, A. F. Yee, J. Vac. Sci. Technol. B 2003, 21, 2749. (b) Y. S. Kim, S. J. Baek, P. T. Hammond, Adv. Mater. 2004, 16, 581.

[204] (a) W. R. Childs, R. G. Nuzzo, J. Am. Chem. Soc. 2002, 124, 13584. (b) H. Ahn, K.J. Lee, A. Shim, J. A. Rogers, R. G. Nuzzo, Nano Lett. 2005, 5, 2533. (c) H. Ahn, K. J. Lee, W. R. Childs, J. A. Rogers, R. G. Nuzzo, J. Appl. Phys. 2006, 100, 084907.

[205] (a) J. Choi, D. Kim, P. J. Yoo, H. H. Lee, Adv. Mater. 2005, 17, 166. (b) J. K. Kim, J. W. Park, H. Yang, M. Choi, J. H. Choi, K. Y. Suh, Nanotechnology 2006, 17, 940. (c) J. K. Kim, K. Y. Suh, Appl. Phys. Lett. 2008, 92, 223107.

[206] J. Zaumseil, M. A. Meitl, J. W. P. Hsu, B. R. Acharya, K. W. Baldwin, Y. L. Loo, J. A. Rogers, Nano Lett. 2003, 3, 1223.

[207] S. Y. Park, Y. H. Noh, H. H. Lee, Appl. Phys. Lett. 2006, 88, 113503.

[208] H. Yoon, T. Kim, S. Choi, K. Y. Suh, M. J. Kim, H. H. Lee, Appl. Phys. Lett. 2006, 88, 254104.

[209] E. Kim, Y. xia, X.-M. Zhao, G. M. Whitesides, Adv. Mater. 1997, 9, 651.

[210] (a) Y. S. Kim, K. Y. Suh, H. H. Lee, Appl. Phys. Lett. 2001, 79, 2285. (b) S. Y. Kim, J. Park, H. H. Lee, Appl. Phys. Lett. 2002, 81, 1011.

[211] (a) W. M. Choi, O. O. Park, Nanotechnology 2004, 15, 135. (b) W. M. Choi, O. O. Park, Appl. Phys. Lett. 2004, 85, 3310.

[212] H. E. Jeong, S. H. Lee, P. Kim, K. Y. Suh, Nano Lett. 2006, 6, 1508.

[213] S. Y. Chou, Q. Xia, Nature Nanotechnology, 2008, 3, 295. 


\section{Supramolecular microcontact printing and dip-pen nanolithography on molecular printboards}

This chapter describes the transfer of functional molecules onto selfassembled monolayers (SAMs) by means of soft and scanning-probe lithographic techniques - microcontact printing $(\mu C P)$ and dip-pen nanolithography (DPN), respectively - and the stability of molecular patterns during competitive rinsing conditions.

The formation of kinetically stable molecular patterns with a lateral resolution of $60 \mathrm{~nm}$ exemplifies the use of $\beta-C D S A M s$ as molecular printboards for the selective immobilization of printboard-compatible guests at the nanometer scale through the use of specific, multivalent supramolecular interactions.

* Parts of this chapter have been published in: C. M. Bruinink, C. A. Nijhuis, M. Péter, B. Dordi, O. CrespoBiel, T. Auletta, A. Mulder, H. Schönherr, G. J. Vancso, J. Huskens, D. N. Reinhoudt, Chem. Eur. J. 2005, 11, 3988 (Copyright (c) 2005 Wiley-VCH Verlag GmbH \& Co. KGaA); T. Auletta, B. Dordi, A. Mulder, A. Sartori, S. Onclin, C. M. Bruinink, M. Péter, C. A. Nijhuis, H. Beijleveld, H. Schönherr, G. J. Vancso, A. Casnati, R. Ungaro, B. J. Ravoo, J. Huskens, D. N. Reinhoudt, Angerw. Chem. Int. Ed. 2004, 43, 369; Angew. Chem. 2004, 116, 373 (Copyright (C) 2004 Wiley-VCH Verlag GmbH \& Co. KGaA). 


\subsection{Introduction}

The ability to transfer functional molecules onto surfaces on the nanometer scale is one of the enabling principles in the field of nanotechnology. Generally, there are two strategies for the transfer of molecules onto surfaces: (i) the use of templates (e.g., patterns of self-assembled monolayers (SAMs) or polymers) to direct and control the selective deposition of molecules from solution; (ii) active deposition onto surfaces by means of a patterning element (e.g., a stamp or a probe). The advantage of the latter patterning strategy is that the transfer of molecules onto surfaces does not rely on expensive photolithographic procedures and does not require processing conditions that are incompatible with several interesting types of functional molecules (e.g., biomolecules) or susceptible to cross-contamination by nonspecific binding. Therefore, direct-patterning strategies in the micro- and nanometer regime such as soft lithography ${ }^{[1,2]}$ and scanning-probe lithographies (SPL) ${ }^{[3]}$ are extensively used for immobilizing functional molecules on surfaces. Examples include microcontact printing $(\mu \mathrm{CP})^{[1]}$ of proteins on glass ${ }^{[4]}$ or of polymers on reactive SAMs on gold ${ }^{[5]}$ and dip-pen nanolithography (DPN) $)^{[6,7]}$ of DNA on gold and glass. ${ }^{[8]}$ However, in most cases, the immobilization of molecules on the surface is accomplished either by covalent binding to the surface, or to SAMs consisting of anchoring molecules, or by nonspecific physisorption.

Supramolecular interactions combine the advantages of chemisorption and physisorption to immobilize functional molecules on surfaces. Supramolecular interactions are directional and specific and allow fine-tuning of the adsorption/ desorption processes at receptor surfaces, ${ }^{[9]}$ which is not feasible for conventional routes of immobilizing molecules on surfaces. Cyclodextrins (CDs) and their derivatives are attractive receptor molecules for application in receptor surfaces, as these molecules have been known to accommodate and form inclusion complexes with various organic guest molecules in aqueous solutions through hydrophobic interactions. ${ }^{[10,11]} \beta-\mathrm{CD}$ adsorbates containing multiple attachment points form SAMs on gold with a high degree of order, in which the recognition sites are pointing toward the outer interface of the monolayer in a quasi-hexagonal lattice. ${ }^{[12,13]}$ This high degree of order and dense packing of the monolayer are prerequisites for the application of these SAMs in sensor applications in general, ${ }^{[14]}$ and specifically in the 
present $\beta$-CD SAMs. ${ }^{[9]}$ These conditions render the (hydrophobic) recognition sites of all $\beta$-CD receptors on the surface essentially identical for guest complexation and minimize nonspecific adsorption.

The interaction strength of small, monovalent guest molecules to these $\beta-C D$ SAMs has been studied by electrochemical impedance spectroscopy (EIS) ${ }^{[13]}$ and surface plasmon resonance (SPR) ${ }^{[15]}$ spectroscopy. Such guest molecules bind to $\beta-\mathrm{CD}$ SAMs with a binding strength that is identical to the intrinsic interaction strength with native $\beta-C D$ in solution. Application of guest molecules that can form multivalent interactions offer a tool to fine-tune the overall interaction strength from reversible to permanent binding to the surface. The use of $\beta$-CD SAMs as molecular printboards ${ }^{[16]}$ exploits this central property in order to position multivalent guests on the surface. For this purpose, a divalent printboard-compatible guest molecule ${ }^{[17]}$ has been specifically designed to study multivalent interactions at the printboard. ${ }^{[18,19]}$ The contrasting binding behavior of this molecule and, in general, multivalent molecules in comparison to monovalent guest molecules is that the former molecules are capable of forming additional interactions and, therefore, have higher overall binding strengths with the $\beta-C D$ surface. The overall interaction strength of this divalent guest molecule to the $\beta$-CD SAM was five orders of magnitude higher than the binding strength of the monovalent guest molecule. The use of multivalent guests such as dendrimers ${ }^{[9]}$ and polymers ${ }^{[20]}$ are known to exhibit even stronger interactions with $\beta$-CD SAMs. The advantage of dendrimers is that the number of interactions with the printboard is tunable by adjusting the dendrimer generation. ${ }^{[21]}$

The successful positioning of functional printboard-compatible guest molecules into molecular patterns on surfaces by supramolecular $\mu \mathrm{CP}$ and supramolecular DPN has been reported previously. ${ }^{[22]}$ The reversibility of supramolecular binding allows, in principle, the preparation of stable molecular patterns (e.g., by printing or writing) under one set of conditions and erasure of these patterns under another set of conditions. This chapter represents the first systematic study that addresses the kinetic stability of molecular patterns on $\beta-\mathrm{CD}$ printboards towards (competitive) rinsing conditions in relation to the number of binding functionalities (i.e., valency). 


\subsection{Results and discussion}

Self-assembled monolayers (SAMs) of the $\beta$-CD heptathioether adsorbate $1 \mathrm{~b}$ (Scheme 3.1) were prepared on gold layers $(20 \mathrm{~nm}$ ) on silicon substrates, as described previously in the literature. ${ }^{[13,23]}$ Scheme 3.1 also depicts different adamantyl- (Ad-) functionalized guest molecules $(2,3,4 \mathrm{~b})$ that were used in the present study for the selective transfer onto the $\beta-\mathrm{CD}$ printboard on gold by supramolecular $\mu \mathrm{CP}$ and/or supramolecular $\mathrm{DPN}^{[22]}$ (Scheme 3.2).

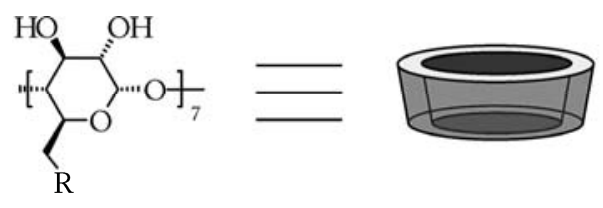

1a $\quad \mathrm{R}=\mathrm{OH}$

$1 \mathrm{~b} \quad \mathrm{R}=\mathrm{NHC}(\mathrm{O})\left(\mathrm{CH}_{2}\right)_{11} \mathrm{~S}\left(\mathrm{CH}_{2}\right)_{11} \mathrm{CH}_{3}$

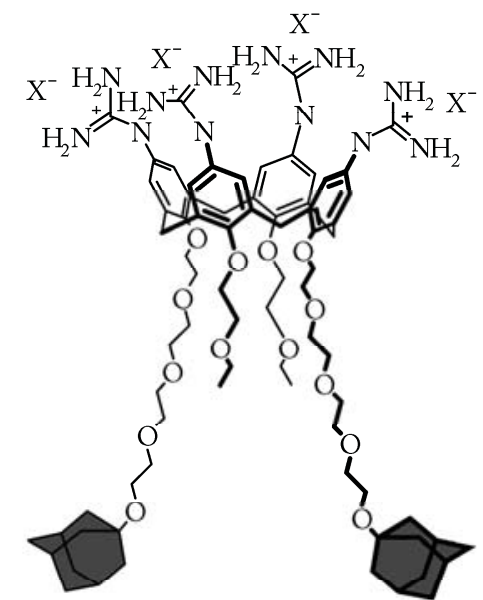

$3 \mathrm{X}=\mathrm{CH}_{3} \mathrm{COO}^{-}$
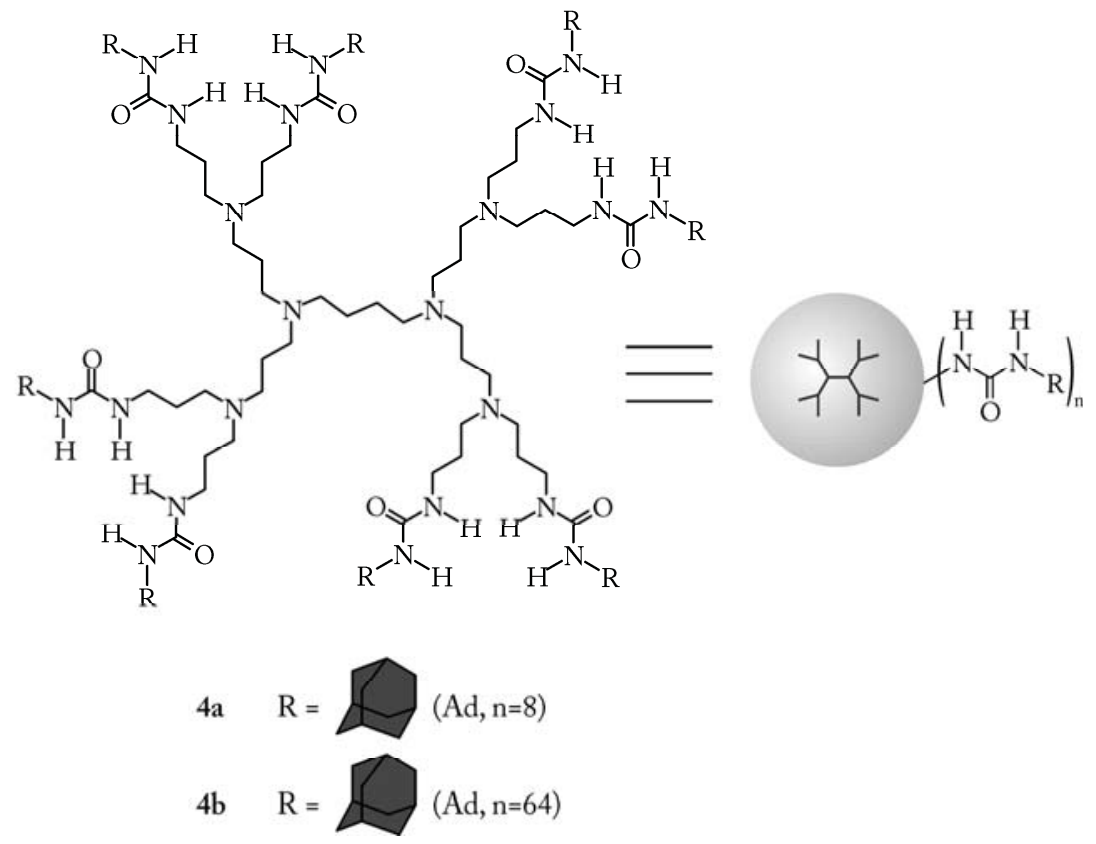

Scheme 3.1 Chemical structures of the receptors, $\beta$-cyclodextrin (1a) and the $\beta$-CD adsorbate for attachment to gold (1b), and of the guests, 1-adamantyl-3-methyl-urea (2), bis(adamantyl)-functionalized calix[4]arene (3), and the fifth-generation adamantyl-functionalized poly(propylene imine) dendrimer (G5-PPI-(Ad) $\left.{ }_{64}\right)(4 \mathrm{~b})$. The chemical structure of the G2-PPI-(Ad) $)_{8}(\mathbf{4 a})$ is shown for convenience. 
on molecular printboards
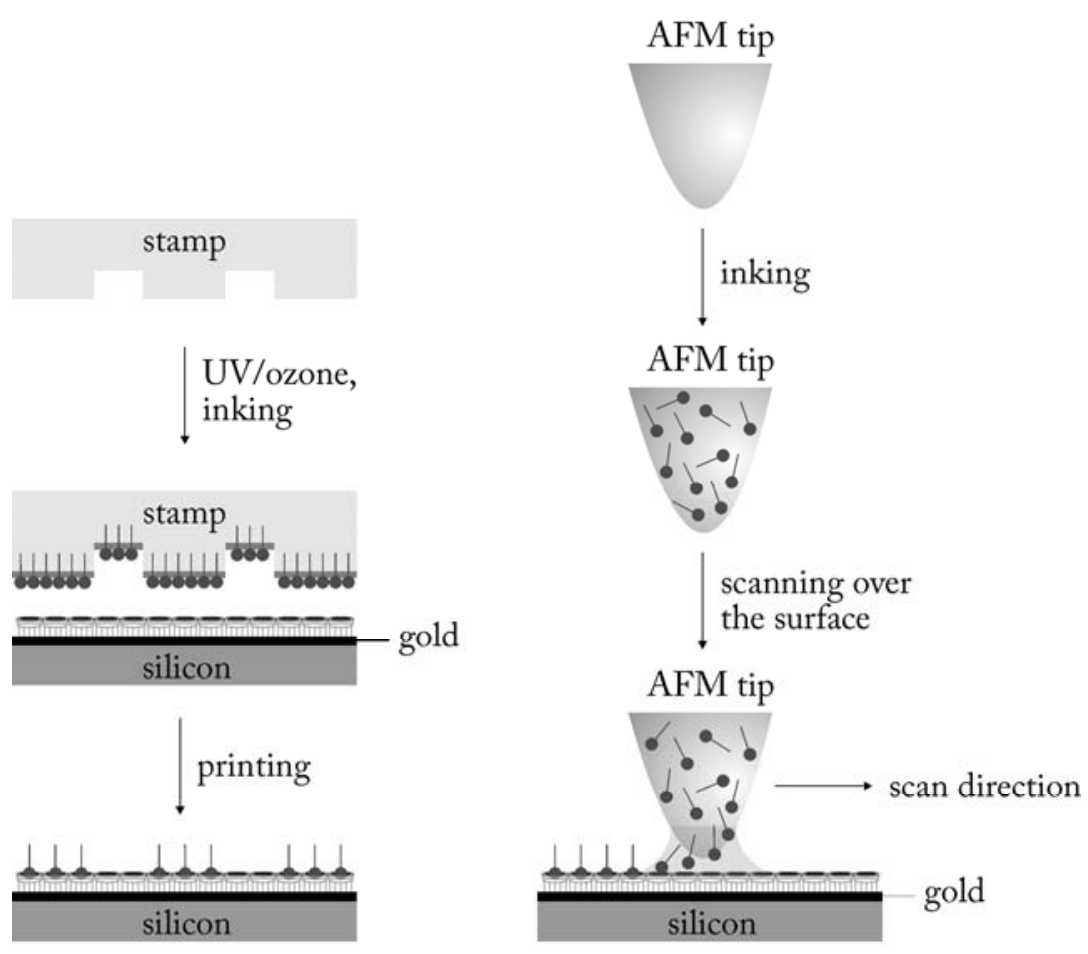

Supramolecular $\mathrm{mCP}$

Supramolecular DPN

Scheme 3.2 Schematic representation of the transfer of printboard-compatible guests on the $\beta$-CD printboard by supramolecular $\mu \mathrm{CP}$ (left) and supramolecular DPN (right).

\subsubsection{Supramolecular microcontact printing - pattern stability}

For the supramolecular printing experiments, poly(dimethylsiloxane) (PDMS) stamps were fabricated by replica molding (REM) of a silicon master structure with periodic lines ( $3 \mu \mathrm{m}$ width, $5 \mu \mathrm{m}$ spacing, $1.2 \mu \mathrm{m}$ depth). Treatment of the PDMS stamps by mild oxidation in a UV/ozone plasma reactor for 60 min was carried out to render the PDMS stamps hydrophilic for subsequent immersion inking in an aqueous solution of the guest for about 15-30 min. Contact-printing experiments were carried out by manually placing the stamp on the surface and leaving the stamp in contact with the surface for 1-2 min. Prior to studying the stability of the resulting molecular patterns of 2,3 , and $4 \mathrm{~b}$ by atomic force microscopy (AFM) friction-force imaging, $\mathrm{X}$-ray photoelectron spectroscopy (XPS) analysis ${ }^{[24]}$ was performed to study the degree of guest deposition by adsorption from solution and by transfer from PDMS stamps (Table 3.1). 
Table 3.1 Elemental analysis by XPS of the $\beta$-CD printboard before and after deposition of divalent guest 3 by adsorption from solution and by $\mu \mathrm{CP}$, and the theoretical XPS values ${ }^{[*]}$ relating to the number of monolayers of 3 present on the printboard.

XPS [percentage composition]

\begin{tabular}{|c|c|c|c|c|c|}
\hline & Guest deposition & $\mathrm{C}(1 \mathrm{~s})$ & $\mathrm{N}(1 \mathrm{~s})$ & $\mathrm{O}(1 \mathrm{~s})$ & $S(2 p)$ \\
\hline \multirow{5}{*}{ 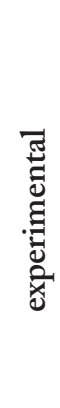 } & no guest & $79.5 \pm 0.9$ & $2.6 \pm 0.5$ & $16.2 \pm 0.4$ & $1.7 \pm 0.1$ \\
\hline & (bare $\beta-C D)$ & & & & \\
\hline & adsorption & $77.8 \pm 0.6$ & $4.0 \pm 0.3$ & $17.1 \pm 0.6$ & $1.2 \pm 0.3$ \\
\hline & $\mu \mathrm{CP}$ & $74.5 \pm 0.7$ & $3.7 \pm 0.2$ & $20.7 \pm 1.0$ & $1.1 \pm 0.2$ \\
\hline & $\mu \mathrm{CP}+$ rinsing & $76.7 \pm 0.5$ & $3.6 \pm 0.4$ & $18.5 \pm 0.9$ & $1.3 \pm 0.1$ \\
\hline \multirow{7}{*}{ 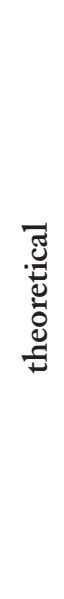 } & \multicolumn{5}{|l|}{ \# monolayers of $3^{[*]}$} \\
\hline & 0 (bare $\beta$-CD) & 81.0 & 2.7 & 13.5 & 2.7 \\
\hline & 1 & 79.2 & 4.1 & 14.4 & 2.2 \\
\hline & 2 & 78.0 & 5.0 & 15.1 & 1.9 \\
\hline & 3 & 77.1 & 5.7 & 15.6 & 1.6 \\
\hline & 4 & 76.4 & 6.3 & 16.0 & 1.4 \\
\hline & 5 & 75.8 & 6.7 & 16.2 & 1.3 \\
\hline
\end{tabular}

The close-to-theoretical XPS values in Table 3.1 confirm the atomic composition of bare $\beta-\mathrm{CD}$ printboards. For the purpose of this study, divalent guest 3 was deposited on the entire surface of the $\beta-\mathrm{CD}$ printboard by printing with featureless PDMS stamps and by adsorption from solution. Physisorption due to electrostatic interactions was ruled out by rinsing the substrates with $50 \mathrm{mM}$ aqueous $\mathrm{NaCl}$ solution $(200 \mathrm{~mL})$ and water $(50 \mathrm{~mL})$. The amounts of guest present on the printboards were estimated by comparing experimental atomic compositions with the corresponding theoretical values. The latter relate to a number of guest monolayers, in which a guest monolayer is defined as the amount of guest that leads to full saturation of available 
host sites by using the highest possible valency. For 3, this corresponds to divalent binding. ${ }^{[18]}$ To correct for the small deviations in atomic composition of the bare $\beta-\mathrm{CD}$ printboard between the experimental and theoretical values, the (absolute) changes in atomic composition were taken for comparison. From the clear trends shown in Table 3.1, the carbon and oxygen data appeared to offer the best estimation.

Approximately one monolayer of guest molecules on the surface (expected: $\Delta \mathrm{C} \%-1.8, \Delta \mathrm{O} \%+0.9)$ was left after adsorption from solution $(\Delta \mathrm{C} \%-1.7, \Delta \mathrm{O} \%+0.9)$, with also the $\mathrm{N}$ and $\mathrm{S}$ changes in close agreement. Conversely, the experimental values indicate that (i) up to 5 guest monolayers (expected: $\Delta \mathrm{C} \%-5.0, \Delta \mathrm{O} \%+4.5$ ) were transferred during physical contact of the stamp with the printboard $(\Delta \mathrm{C} \%-5.2 \%, \Delta \mathrm{O} \%+2.7)$, and that (ii) $1-2$ guest monolayers $(\Delta \mathrm{C} \%-2.8, \Delta \mathrm{O} \%+2.3)$ were remaining after rinsing the substrate with $50 \mathrm{mM}$ aqueous $\mathrm{NaCl}(200 \mathrm{~mL})$ (expected: $\Delta \mathrm{C} \%-3.0, \Delta \mathrm{O} \%+1.6)$. Submonolayer coverages were the result after rinsing with competitive rinsing solutions (e.g., 1-10 $\mathrm{mM}$ native $\beta-\mathrm{CD}$ at $\mathrm{pH} 2$, data not shown).

The stability of the molecular patterns of a series of different guest molecules was studied by comparing the patterns on the $\beta-\mathrm{CD}$ printboard directly after $\mu \mathrm{CP}$ with the patterns after rinsing with water, $1 \mathrm{mM} \beta-\mathrm{CD}$, or $10 \mathrm{mM} \beta-\mathrm{CD}$ solutions $(200 \mathrm{~mL}$; Fig. 3.1). By contact-mode AFM imaging of the $\beta-\mathrm{CD}$ printboard after $\mu \mathrm{CP}$, clear patterns of different friction contrast were seen, confirming the transfer of the guest molecules from the PDMS stamp onto the substrates (Figs. 3.1a, 3.1d and 3.1g). ${ }^{[25]}$ Complete removal of the patterns of monovalent guest 2 from the $\beta$-CD printboard was already induced by rinsing with water, as shown by the disappearance of the friction contrast (Figs. 3.1a-b). However, this rinsing procedure did not affect the patterns of divalent guest 3 (Figs. 3.1d and 3.1e). Additionally, even rinsing with substantial amounts of $10 \mathrm{mM} \beta-\mathrm{CD}$ solution did not result in complete removal of the patterns of 3 (Fig. 3.1f), even though competition is known to enhance multivalent dissociation kinetics. ${ }^{[26]}$ The high stability of the molecular patterns of 3 to rinsing with $10 \mathrm{mM} \beta-\mathrm{CD}$ solution stems from the high overall interaction strength if a guest is capable of forming more than one supramolecular interaction with $\beta$-CDs on the printboard. ${ }^{[18,19]}$ On a qualitative basis, it is clear from the relative friction contrast changes before and after rinsing with $10 \mathrm{mM} \beta$-CD solution, that molecular patterns 
of multivalent guest $4 \mathrm{~b}$ (Figs. $3.1 \mathrm{~g}$ and $3.1 \mathrm{i}$ ) are even more stable towards competitive rinsing than the divalent guest 3 (Figs. 3.1d and 3.1f).
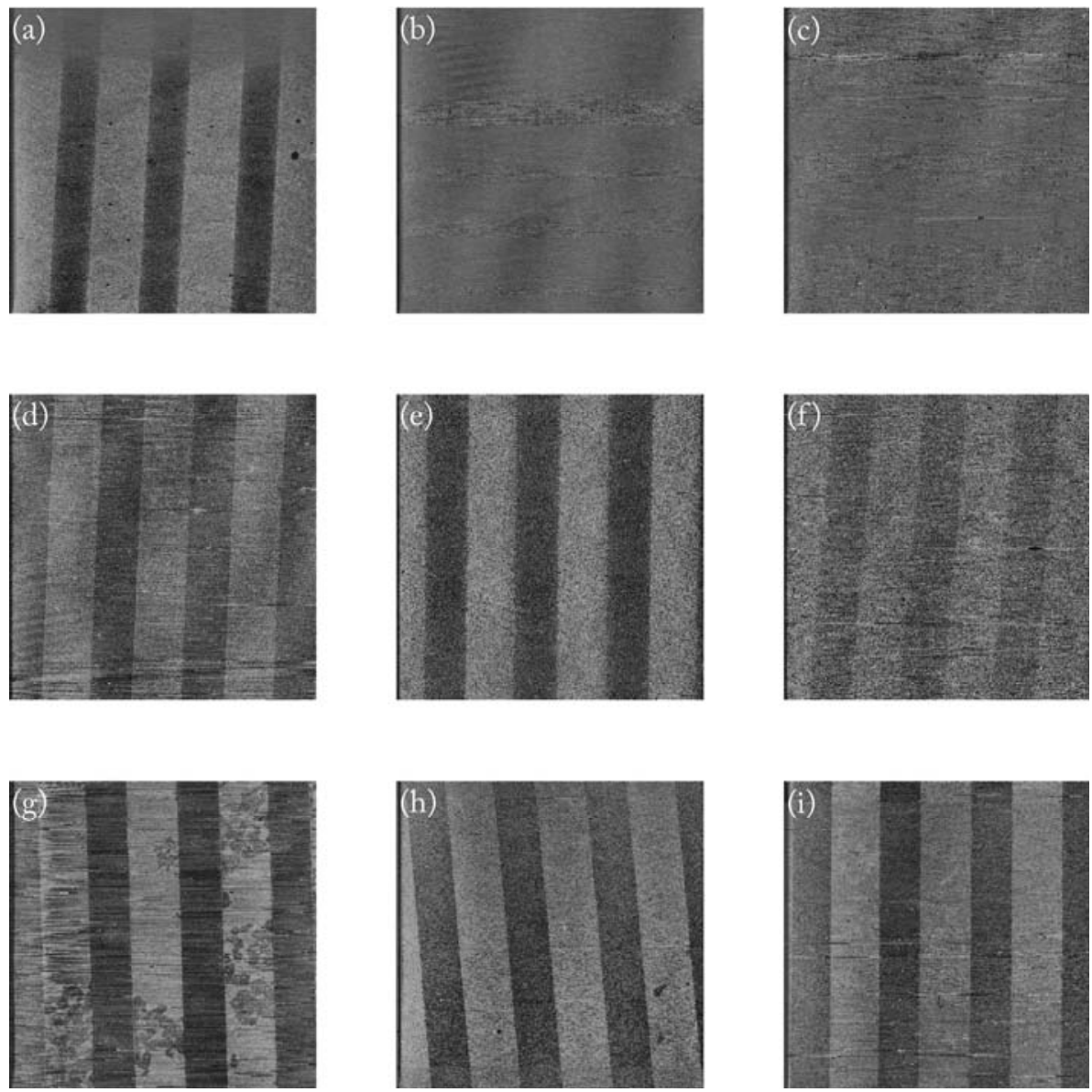

Figure 3.1 Contact-mode (CM) AFM friction images of patterns of monovalent guest 2 (top), divalent guest 3 (center), and multivalent guest $4 \mathrm{~b}$ (bottom) showing patterns present on the $\beta$-CD printboard (image sizes $50 \mu \mathrm{m} \times 50 \mu \mathrm{m}$; friction forces [a.u.] increase from dark to bright contrast): after $\mu \mathrm{CP}$ of the guests (a, d, g) (brighter areas), after rinsing with water (b, e, h) and after rinsing with $10 \mathrm{mM} \beta-\mathrm{CD}$ at $\mathrm{pH} 2$ (c, f, i).

The fact that the transfer of $4 \mathrm{~b}$, as its per- $\beta-\mathrm{CD}$ complex, ${ }^{[27]}$ by $\mu \mathrm{CP}$ onto the $\beta-C D$ printboard is successful and the fact that its patterns are very stable during competitive rinsing emphasize its strongly multivalent nature. The association of this type of multivalent guest to the printboard most probably occurs through seven Ad-CD interactions, as has been recently shown for similar ferrocenyl-functionalized dendrimers. ${ }^{[21]}$ AFM height measurements (not shown) illustrate that multilayers of guest $4 \mathrm{~b}$ (feature height about $3 \mathrm{~nm}$ ) were transferred onto the $\beta$-CD printboard during $\mu \mathrm{CP}$. Friction contrast variations within the areas of transfer (Fig. 3.1g) 
correlate with nonuniformities in layer thickness. Additionally, rinsing with $10 \mathrm{mM}$ $\beta-\mathrm{CD}$ solution was found to effectively suppress physisorption, resulting in $\sim 1$ monolayer of $4 \mathrm{~b}$ on the $\beta-\mathrm{CD}$ printboard (feature height after rinsing was about $1 \mathrm{~nm})$. Apparently, the interaction strength of guest $4 \mathrm{~b}$ with the $\beta-\mathrm{CD}$ printboard is sufficient to apply the present receptor surfaces as molecular printboards for the printing of stable molecular patterns of multivalent guests by supramolecular $\mu \mathrm{CP}$.

Electrochemical impedance spectroscopy (EIS) measurements were carried out in order to obtain more information on the degree of deposition of guest $4 \mathrm{~b}$ on the printboard. For this purpose, multivalent guest $4 \mathrm{~b}$ was deposited on the entire surface of $\beta$-CD printboards by printing with featureless PDMS stamps and by adsorption from solution. The effect of rinsing with $10 \mathrm{mM} \mathrm{CD}$ solution $(200 \mathrm{~mL})$ at $\mathrm{pH} 2$ and water $(50 \mathrm{~mL})$ at $\mathrm{pH} 2$ was also taken into account (Table 3.2).

Table 3.2 EIS results of the $\beta$-CD printboard before and after deposition of multivalent guest $4 \mathrm{~b}$ by adsorption from solution and by $\mu \mathrm{CP}$.

\begin{tabular}{lc}
\hline Guest deposition & $\begin{array}{c}\mathrm{R}_{\mathrm{CT}}{ }^{[*]} \\
{[\mathrm{k} \Omega]}\end{array}$ \\
\hline no guest & 42 \\
adsorption & 8.4 \\
$\mu \mathrm{CP}$ & 75 \\
$\mu \mathrm{CP}+$ rinsing & 7.3 \\
\hline
\end{tabular}

$\overline{[*]}$ Charge-transfer resistance towards $\left[\mathrm{Fe}(\mathrm{CN})_{6}\right]^{4-} /\left[\mathrm{Fe}(\mathrm{CN})_{6}\right]^{3-}$.

The initial charge-transfer resistance $\left(\mathrm{R}_{\mathrm{CT}}\right)$ of the $\beta$-CD printboard $(42 \mathrm{k} \Omega)$ towards the negatively charged $\left[\mathrm{Fe}(\mathrm{CN})_{6}\right]^{4-} /\left[\mathrm{Fe}(\mathrm{CN})_{6}\right]^{3-}$ reporter redox couple is similar to $\mathrm{R}_{\mathrm{CT}}$ values reported previously in the literature ${ }^{[13]}$ and reflects a high degree of order of this monolayer to block the redox current to the gold efficiently. The presence of guest $4 b$ on the printboard is known to significantly decrease this $\mathrm{R}_{\mathrm{CT}}$ since electrostatic attraction of the anionic reporter redox couple with the positively charged dendritic core facilitates the oxidation/reduction at the electrode surface. ${ }^{[9]}$ This was found to be the case for deposition of $4 \mathrm{~b}$ onto the printboard by adsorption from solution and by $\mu \mathrm{CP}$ and subsequent rinsing $(7-8 \mathrm{k} \Omega)$, resulting in monolayer 
coverages of guest molecules on the printboard. Conversely, the two-fold increase in the $\mathrm{R}_{\mathrm{CT}}$ value upon deposition by $\mu \mathrm{CP}$ (without rinsing) suggests, in agreement with AFM analysis, the transfer of multilayers of guest $4 \mathrm{~b}$ onto the $\beta-\mathrm{CD}$ printboard during $\mu \mathrm{CP}$. In this case, the increase of the layer thickness of guest molecules on top of the printboard impedes the redox current to the electrode surface and, therefore, results in a higher $\mathrm{R}_{\mathrm{CT}}$ value.

To rule out any interference from nonspecific interactions on the $\beta-\mathrm{CD}$ printboard, printing experiments under the same conditions were carried out on SAMs of 11-mercapto-undecanol (OH SAMs) (Figure 3.2).
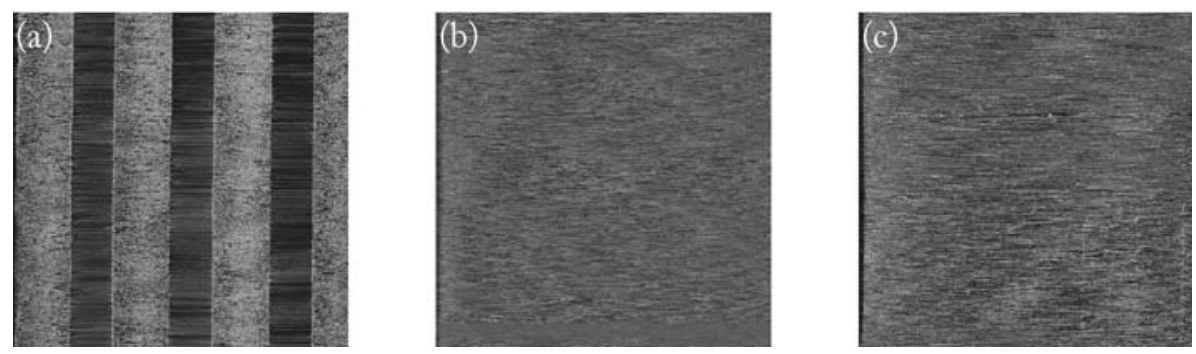

Figure 3.2 CM AFM friction images of micrometer-size line patterns of multivalent guest $4 \mathrm{~b}$ (brighter areas) on 11-mercaptoundecanol SAMs (bottom) showing patterns present on the $\beta$-CD printboard (image sizes $50 \mu \mathrm{m} \times 50 \mu \mathrm{m}$; friction forces [a.u.] increase from dark to bright contrast): after $\mu \mathrm{CP}$ of the guests (brighter areas) (a), after rinsing with $1 \mathrm{mM} \beta-\mathrm{CD}$ at $\mathrm{pH} 2$ (b) and after rinsing with $10 \mathrm{mM} \beta-\mathrm{CD}$ at $\mathrm{pH} 2$ (c).

$\mathrm{OH}$ SAMs were chosen as reference layers, since these SAMs have a similar polarity as SAMs of $\beta-C D$, but are incapable of forming inclusion complexes with the guest molecules. Figs. 3.1g-i and 3.2a-c illustrate the concept of the molecular printboard by the difference in stability of the patterns of multivalent guest $4 \mathrm{~b}$ on the $\beta-\mathrm{CD}$ printboard and on $\mathrm{OH}$ SAMs upon subsequent rinsing with different concentrations of $\beta-C D$ solutions.

Again, patterns with a clear friction contrast are apparent in contact-mode AFM images of the $\mathrm{OH}$ SAM after $\mu \mathrm{CP}$. This indicates that the transfer of guest molecules from the stamp onto the $\beta$-CD printboard does not rely on specific interactions, but that physical contact is sufficient to generate patterns of molecules (Figs. 3.1g and 3.2a). The requirement of specific supramolecular interactions for the stability of the molecular patterns on the printboard is, however, evident from the complete removal of the patterns of multivalent guest $4 \mathrm{~b}$ from the $\mathrm{OH}$ SAMs upon rinsing with $1 \mathrm{mM}$ 
and $10 \mathrm{mM} \beta-\mathrm{CD}$ solutions (Figs. 3.2b and 3.2c), a result confirming physisorption in this case. Patterns of $4 \mathrm{~b}$ on the $\beta-\mathrm{CD}$ printboards were stable even after rinsing with substantial amounts of a $10 \mathrm{mM} \beta-\mathrm{CD}$ solution (Fig. 3.1i).

Poly(propylene imine) (PPI) dendrimers such as $\mathbf{4 b}$ have already been employed as nanocontainers for dye molecules on the $\beta-\mathrm{CD}$ printboard ${ }^{[28]}$ and as water-soluble nanoreactors for the formation of gold nanoparticles in solution. ${ }^{[29]}$ In the present study, the latter dendrimer-stabilized gold nanoparticles were exploited to facilitate the fabrication of metal patterns on the printboard by incorporating an additive technique, namely electroless deposition (ELD) into the present supramolecular patterning strategies.

\subsubsection{Electroless deposition - fabrication of metal patterns on the printboard}

$\mathrm{ELD}^{[30]}$ is a routine and cost-effective fabrication technique for the selective deposition of metal on surfaces. It is an autocatalytic redox process that comprises the reduction and deposition of metal ions (as the source of metal) from solution to a substrate in the presence of a reducing agent. To initiate ELD of metal on surfaces, the only requirement is a surface containing a catalyst. For this purpose, G5-PPI dendrimer-stabilized gold nanoparticles were prepared by a literature procedure, ${ }^{[29]}$ and subsequently transferred as catalysts for ELD onto the molecular printboard by supramolecular $\mu \mathrm{CP}$. Fig. 3.3 shows AFM height images of this printboard before and after immersion in a copper ELD bath for $5 \mathrm{~min}$ and indicates average heights of 2 and $64 \mathrm{~nm}$ (see cross-section AFM analysis in Fig. 3.3), respectively. The fact that the patterns of dendrimer-stabilized nanoparticles were stable during competitive rinsing with $10 \mathrm{mM} \beta-\mathrm{CD}(200 \mathrm{~mL}$ ) at $\mathrm{pH} 2$ (Fig. 3.3a) supports the idea that the gold particles reside inside the dendrimers ${ }^{[29]}$ and that multivalent interactions of several Ad functionalities at the outer interface of the nanoparticle with the printboard account for the pattern stability. As argued before, the dense, insulating shell of supramolecular Ad- $\beta-\mathrm{CD}$ complexes protects the nanoparticles inside the dendrimer from coagulation. ${ }^{29]}$ The ELD experiment shown here illustrates that these nanoparticles are still accessible to act as catalytic centers for the ELD of copper. Fig. 3.3b also illustrates the high selectivity for copper deposition in the target areas containing the dendrimer-stabilized gold nanoparticles. The direct placement of these 
catalytic centers and the protective nature of the $\beta$-CD SAM for the underlying gold substrate accounts for this observation.
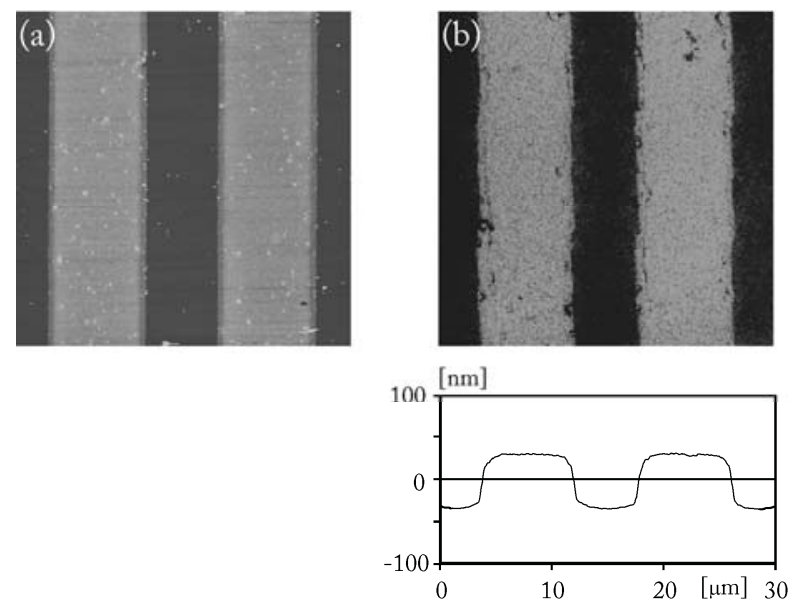

Figure 3.3 CM AFM height images of patterns on the $\beta$-CD printboard (image sizes $30 \mu \mathrm{m} \times 30 \mu \mathrm{m}$ ): (a) patterns of G5-PPI dendrimer-stabilized gold nanoparticles after supramolecular $\mu \mathrm{CP}$ and subsequent rinsing with $10 \mathrm{mM} \beta-\mathrm{CD}$ solution at $\mathrm{pH} \mathrm{2,} \mathrm{and} \mathrm{(b)} \mathrm{the} \mathrm{corresponding} \mathrm{copper} \mathrm{structures} \mathrm{by} \mathrm{subsequent}$ electroless deposition for $5 \mathrm{~min}$.

The combination of ELD and $\mu \mathrm{CP}$ has been reported previously to offer a routine and cost-effective fabrication technique for metal patterns. ${ }^{[31,32]}$ However, the distinct difference between the present work and these previous reports is the use of multivalent supramolecular interactions between the catalyst and the surface that can subsequently serve as a strong connection between the metal patterns and the substrate.

\subsubsection{Supramolecular DPN - writing local molecular patterns at the sub-100 $\mathrm{nm}$ scale}

DPN is a promising lithographic technique that allows the extension of the supramolecular patterning strategy on the printboard to the sub-100 nm scale (Scheme 3.2). ${ }^{[6,7]}$ Previously, it has been shown that patterns of 3 on the $\beta-C D$ printboard transferred by DPN are similar to those prepared by supramolecular $\mu \mathrm{CP}$, with regard to the issues of ink transfer and pattern stability upon rinsing. ${ }^{[22]}$ To perform DPN, wet inking of clean silicon nitride AFM tips was done by immersion for $15 \mathrm{~min}$ in an aqueous solution of the guest. The writing experiments on $\beta$-CD printboards were carried out in ambient atmosphere $\left(\mathrm{T}=25^{\circ} \mathrm{C}\right.$, relative humidity $=$ 40-50\%) by scanning the tip in contact mode along a line on the substrate for a 
certain period of time. To investigate the potential of supramolecular DPN, the lateral resolution was investigated by writing patterns with different width-to-length ratios (Fig. 3.4a). ${ }^{[33]}$
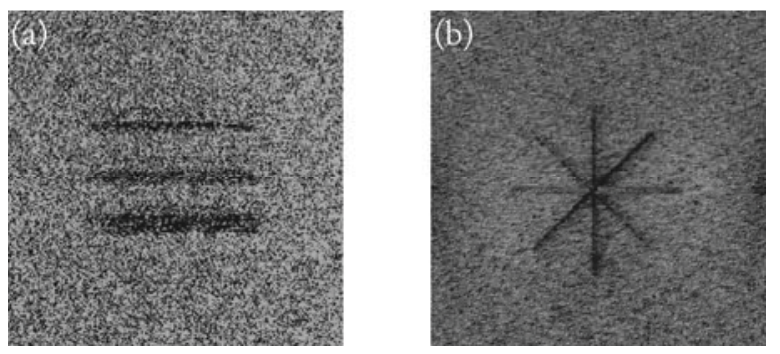

Figure 3.4 CM AFM friction images of patterns present on the $\beta$-CD printboard: (a) an array of lines, $7 \mu \mathrm{m}$ long with mean widths ( \pm standard deviation) of $290 \pm 20 \mathrm{~nm}, 480 \pm 30 \mathrm{~nm}$, and $880 \pm 50 \mathrm{~nm}$, of divalent guest 3 after scanning the tip at a velocity of $\sim 21 \mu \mathrm{m} / \mathrm{s}$ across the $\beta$-CD printboard for $10 \mathrm{~min}$ for each line (image size $15 \mu \mathrm{m} \times 15 \mu \mathrm{m}$; friction forces [a.u.] increase from dark to bright contrast), and (b) an array of lines, $3 \mu \mathrm{m}$ long with average widths of approximately $60 \pm 20 \mathrm{~nm}$, of multivalent guest $4 \mathrm{~b}$ after scanning the tip at a velocity of $\sim 4 \mu \mathrm{m} / \mathrm{s}$ across the $\beta$-CD printboard for $10 \mathrm{~min}$ for each line (image size $6 \mu \mathrm{m} \times 6 \mu \mathrm{m}$; friction forces [a.u.] increase from dark to bright contrast). Readout of the patterns was done with the same tip by increasing the scan size and the scan velocity (approximately 15 times the writing speed).

Fig. 3.4a shows a friction AFM image of a pattern of 3 consisting of three lines, $7 \mu \mathrm{m}$ long with targeted widths of $220 \mathrm{~nm}, 440 \mathrm{~nm}$, and $880 \mathrm{~nm}$ (top to bottom), respectively, written by DPN on the $\beta-\mathrm{CD}$ printboard over $10 \mathrm{~min}$ for each line. Inversion of the relative friction contrast in the image in comparison to the friction contrasts seen in Figs. 3.1 and 3.2 is probably related to the different character of the contacting surfaces (i.e., ink-containing tip versus bare tip), as was shown before for similar $\beta-\mathrm{CD}$ systems, ${ }^{[22]}$ as well as for poly(amidoamine) (PAMAM) dendrimers on other SAMs. ${ }^{[34]}$

Overinking during the writing stage as well as the effect of instrumental drifts are parameters that could cause the pattern broadening in this particular experiment to be more significant at high-resolution writing (ca. 30\% for the $220 \mathrm{~nm}$-wide line, 10\% for the $440 \mathrm{~nm}$-wide line). The sub-100 nm writing capability of supramolecular DPN is shown in Fig. 3.4b with the array of lines of guest $4 \mathrm{~b}$ on the printboard at a resolution of $60 \mathrm{~nm}$. In addition to writing periodic line patterns, this specific pattern was written in four stages by adjusting the scan angle of the tip by $45^{\circ}$ each time with respect to the previous line. In this particular experiment, gold-coated tips containing a poly(ethylene 
glycol) SAM were used to allow a better transfer of $4 \mathrm{~b}$ to the printboard. Scanning with a bare silicon nitride AFM tip over the $\beta-C D$ printboard under the same experimental conditions did not form any visible pattern, thus proving that pattern formation arises from the transfer of guest molecules and not from mechanical forces applied by the tip to the printboard.

\subsection{Conclusions}

The results in this chapter demonstrate the successful application of micro- and nanopatterning strategies, supramolecular $\mu \mathrm{CP}$ and supramolecular $\mathrm{DPN}$, respectively, that employ the concept of the molecular printboard.

Supramolecular $\mu \mathrm{CP}$ with guests of different valencies was carried out on the $\beta-\mathrm{CD}$ printboard for testing the stability of the patterns during rinsing with substantial amounts of water and competitive aqueous solutions. It was found that all kinetic stabilities can be seen: rapid removal by water in the case of a monovalent guest, slower partial removal by competitive rinsing in the case of a divalent guest, and complete stability for truly multivalent guests. Interference of nonspecific interactions in the formation of molecular patterns on the printboard was ruled out by the complete removal of the molecular pattern during mild rinsing conditions on reference OH SAMs. Clearly, the novelty of the present patterning strategy over existing fabrication strategies for molecular patterns on substrates lies in the tunable pattern stability of printboard-compatible guests on the molecular printboard through the use of specific, multivalent supramolecular interactions.

Supramolecular DPN was exploited on the $\beta-\mathrm{CD}$ printboard to extend the supramolecular patterning strategy into the sub-100 $\mathrm{nm}$ range, as shown by the nanoscale patterns with $60 \mathrm{~nm}$ line width.

ELD of metal patterns on the printboard demonstrates that molecular printboards constitute a useful tool for the fabrication and application of supramolecular architectures on surfaces.

\subsection{Experimental}

Chemicals and materials: 11-Mercaptoundecanol, native $\beta$-cyclodextrin, sulfuric acid $\left(\mathrm{H}_{2} \mathrm{SO}_{4}\right)$, hydrogen peroxide $\left(\mathrm{H}_{2} \mathrm{O}_{2}\right)$, methylamine, 1-adamantyl isocyanate, $\mathrm{NaCl}$, 
$\mathrm{CuSO}_{4}, \mathrm{Na}_{2} \mathrm{SO}_{4}, \mathrm{NaOH}$, formaldehyde, sodium formate, ethylenediaminetetraacetate sodium salt ( $\mathrm{Na}_{4} \mathrm{EDTA}$ ), toluene, chloroform, ethanol, dichloromethane, and diethylether were purchased from commercial sources in the highest available purity and used without further purification. A PDMS prepolymer kit (Sylgard 184 silicone elastomer) including PDMS curing agent (Sylgard silicone elastomer curing agent) was purchased from the Dow Corning Corporation. $100 \mathrm{~mm}$-type OSP silicon wafers were purchased from Okmetic and the $20 \mathrm{~nm}$ gold-on-silicon wafers from Ssens BV (Hengelo, The Netherlands).

The synthesis of the $\beta$-CD heptathioether adsorbate $\mathbf{1 b}$ and the preparation of SAMs on gold substrates were reported previously. ${ }^{[13,23]}$ The synthesis of the fifthgeneration adamantyl-terminated poly(propylene imine) dendrimer (G5-PPI-(Ad) $\left.{ }_{64}\right)$ $4 \mathrm{~b}$ and its per- $\beta-\mathrm{CD}$ complex were carried out according to literature procedures. ${ }^{[28]}$

1-Adamantyl-3-methyl-urea (2): A solution of methylamine (0.105 g, $3.4 \mathrm{mmol})$ in chloroform $(20 \mathrm{~mL})$ was added to 1-adamantyl isocyanate $(0.5 \mathrm{~g}, 2.8 \mathrm{mmol})$. This mixture was stirred overnight at room temperature under argon. The solvent was removed under vacuum. Subsequently, diethyl ether was added to the residue to isolate the product as a white solid $(0.260 \mathrm{~g}, 1.25 \mathrm{mmol} ; 45 \%) .{ }^{1} \mathrm{H}$ NMR (300 $\mathrm{MHz}$, $\mathrm{CDCl}_{3}$ ): 4.13 (br s, 1H), 4.09 (br s, $\left.1 \mathrm{H}\right), 2.71$ (d, $\left.J=5.1 \mathrm{~Hz}, 2 \mathrm{H}\right), 2.09-2.03$ (m, 3H; $\left.\mathrm{CH}_{2} \mathrm{CHCH}_{2}[\mathrm{Ad}]\right), \quad 1.98-1.94$ (m, 6H; $\left.\mathrm{CHCH}_{2} \mathrm{C}[\mathrm{Ad}]\right), \quad 1.68-1.64 \quad(\mathrm{~m}, \quad 6 \mathrm{H}$; $\left.\mathrm{CHCH}_{2} \mathrm{CH}[\mathrm{Ad}]\right) ;{ }^{13} \mathrm{C} \mathrm{NMR}\left(100 \mathrm{MHz}, \mathrm{CDCl}_{3}\right)$ : 158.24, 50.75, 42.58, 36.48, 29.60, 26.89; FAB-MS: $m / z 209.2\left([\mathrm{M}+\mathrm{H}]^{+}\right.$, calcd. 209.2).

Substrate and monolayer preparation: Prior to use, all glassware was cleaned by immersion in piraña (3:1 ratio of conc. $\mathrm{H}_{2} \mathrm{SO}_{4}$ and $33 \mathrm{wt} \% \mathrm{H}_{2} \mathrm{O}_{2}$ ) for $30 \mathrm{~min}$ and rinsing with substantial amounts of water. Gold substrates of $1 \mathrm{~cm} \times 1 \mathrm{~cm}$ were cleaned by oxygen plasma treatment for $5 \mathrm{~min}$ and the resulting oxide layer was reduced by dipping the substrates for $10 \mathrm{~min}$ in absolute EtOH. ${ }^{[35]}$ Subsequently, the clean gold substrates were immersed in an adsorbate solution (0.1-1.0 mM) with a minimal delay of $16 \mathrm{~h}$. After incubation, the substrates were rinsed with substantial amounts of dichloromethane, ethanol, and water.

Supramolecular microcontact printing: Stamps were fabricated by casting a 10:1 (v/v) mixture of PDMS and curing agent (Sylgard 184, Dow Corning) against a silicon master. Curing for $1 \mathrm{~h}$ at $60{ }^{\circ} \mathrm{C}$ and release of the PDMS stamps was done at this 
curing temperature to avoid buildup of tension due to thermal shrinkage. ${ }^{[36]}$ PDMS stamps were left in the oven at $60{ }^{\circ} \mathrm{C}$ for at least $18 \mathrm{~h}$ to ensure complete curing.

By nature, PDMS is very hydrophobic $\left(\theta_{\mathrm{adv}}=120^{\circ}\right)$ and in order to transfer polar inks oxidation of the stamps is essential. Oxidation of PDMS stamps was carried out in a commercial UV/ozone plasma reactor (Ultra-Violet Products Inc., model PR-100) for $60 \mathrm{~min}$ at a distance of about $2 \mathrm{~cm}$ from the plasma source. This type of reactor contains a low-pressure mercury UV light operating with UV emissions at $185 \mathrm{~nm}$ $\left(1.5 \mathrm{~mW} \cdot \mathrm{cm}^{-2}\right)$ and $254 \mathrm{~nm}\left(15 \mathrm{~mW} \cdot \mathrm{cm}^{-2}\right)$ to generate molecular oxygen. UV/ozone (UVO) treatment ${ }^{[37]}$ of the PDMS stamp was chosen over other techniques ${ }^{[38,39,40]}$ because this milder treatment results in similar changes of the surface chemical properties of the PDMS as oxygen plasma treatment and in surfaces that were found to be more resistant towards mechanical stress (i.e., crack formation ${ }^{[41]}$ ) during $\mu \mathrm{CP}$. The PDMS stamps were kept hydrophilic by immersing the stamps in an aqueous ink solution immediately after UVO treatment. ${ }^{[42]}$ Hydrophobic recovery of PDMS after the UVO treatment ${ }^{[43]}$ restricts the use of this type of surface modification for $\mu \mathrm{CP} .^{[44]}$ Contact angle measurements over time illustrate that PDMS was moderately hydrophilic $\left(\theta_{\mathrm{adv}}=68^{\circ}\right)$ after the UVO exposure and that hydrophobic recovery was insignificant for at least several hours $\left(\theta_{\mathrm{adv}} \approx 10^{\circ}\right.$ after $\left.4 \mathrm{~h}\right)$ in the case the stamp was kept under water. Additionally, this hydrophobic recovery was found to level off, as seen by the contact angle increase of about $4^{\circ}$ within the next 20 h. For reproducibility, all printing experiments were carried out within the first $4 \mathrm{~h}$ after UVO treatment, without reactivation of the stamps.

Subsequently, the ink was applied on the stamps by immersion into the adsorbate solution (0.1-1.0 $\mathrm{mM}$ in hydrophobic constituents) for 15-30 min. After withdrawal from the solution and drying under a continuous stream of nitrogen for $1 \mathrm{~min}$, the stamps were manually applied onto the substrate and left in contact with the surface for 1-2 min onto the substrate (the $\beta$-CD printboard or reference 11-mercapto-1undecanol SAM). Re-inking was carried out after each printing step. Finally, the substrates were systematically rinsed with $200 \mathrm{~mL}$ of aqueous solutions of native $\beta-\mathrm{CD}(1$ or $10 \mathrm{mM}$ at $\mathrm{pH}$ 2), $\mathrm{NaCl}(50 \mathrm{mM})$ or Milli-Q water. Additional rinsing with Milli-Qwater was done in the first two cases in order to remove any excess of $\beta-\mathrm{CD}$ or salt on the printboard. 
on molecular printboards

Supramolecular DPN: $\mathrm{Si}_{3} \mathrm{~N}_{4}$ tips were cleaned in chloroform overnight and the ink was applied onto the tip by immersion into the adsorbate solution $(0.5-1.0 \mathrm{mM}$ in hydrophobic constituents) with minimal delay of $15 \mathrm{~min}$. After withdrawal and drying, the tip was scanned in contact mode across the surface of the $\beta$-CD printboard for a certain period of time $\left(\mathrm{T}=25{ }^{\circ} \mathrm{C}\right.$, relative humidity $\left.=40-50 \%\right)$. The written patterns were recorded with the same tip by increasing the scan size and the scan velocity (ca. 15 times the writing speed).

Electroless deposition (ELD) of copper: To trigger the ELD of $\mathrm{Cu}$ on the printboard, G5-PPI dendrimer-stabilized gold nanoparticles were prepared according to the literature procedure ${ }^{[29]}$ and transferred onto the printboard by conducting supramolecular $\mu \mathrm{CP}$. After rinsing with $200 \mathrm{~mL}$ of aqueous solutions of native $\beta-\mathrm{CD}$ (10 $\mathrm{mM}$ at $\mathrm{pH}$ 2) and Milli-Q, the substrate was immersed in the copper ELD bath containing $40 \mathrm{mM} \mathrm{CuSO}, 140 \mathrm{mM} \mathrm{Na} \mathrm{SO}_{4}, 120 \mathrm{mM} \mathrm{Na}{ }_{4}$ EDTA, $300 \mathrm{~mm}$ $\mathrm{NaHCOO}$, and $30 \mathrm{mM} \mathrm{HCHO}$ at $\mathrm{pH}$ 13. After taking the substrate out of the ELD bath, the substrate was rinsed with Milli-Q and dried under a continuous stream of nitrogen for $1 \mathrm{~min}$.

Characterization: AFM analyses were carried out with a NanoScope III multimode AFM (Veeco/Digital Instruments, Santa Barbara, CA, USA) equipped with a J-scanner, in contact mode using $\mathrm{Si}_{3} \mathrm{~N}_{4}$ cantilevers (Nanoprobes, Veeco/Digital Instruments) with a nominal spring constant of $0.1 \mathrm{~N} \cdot \mathrm{m}^{-1}$. To ensure maximum sensitivity for lateral forces in the friction-force images, the sample was scanned in $90^{\circ}$ with respect to the long axis of the cantilever. AFM imaging was performed in ambient atmosphere $\left(25^{\circ} \mathrm{C}\right.$, relative humidity $\left.=40-50 \%\right)$.

XPS analyses were carried out with a Physical Electronics Quantum2000 equipped with an $\mathrm{Al} \mathrm{K}$ monochromatic excitation source (source energy $=1486.7 \mathrm{eV}$, take-off angle set to $30^{\circ}$ ), a spherical sector analyzer and a multichannel plate detector (16 detector elements). For the survey scan (pass energy of $117 \mathrm{eV}$ ), the $\mathrm{X}$-ray beam was set to high power mode $(100$ Watt per $100 \mu \mathrm{m})$ to scan a total area of $1000 \mu \mathrm{m} \times 500 \mu \mathrm{m}$; for element scans (pass energy of $29.35 \mathrm{eV}$ ), the $\mathrm{X}$-ray beam was set to $25 \mathrm{~W}$ per $100 \mathrm{~m}$ to scan a total scan area of $1000 \mu \mathrm{m} \times 500 \mu \mathrm{m}(298 \mathrm{~K}$ and $1 \cdot 10^{-8}-3 \cdot 10^{-8}$ Torr). The sensitivity factors used for $\mathrm{C}, \mathrm{N}, \mathrm{O}$ and $\mathrm{S}$ for calculating the 
atomic concentration are respectively $0.314,0.499,0.733$ and 0.717 . The hydrocarbon $\mathrm{C}(1 \mathrm{~s})$ signal at $284.8 \mathrm{eV}$ was used as the reference to correct for surface charging.

Electrochemical impedance spectroscopy measurements were carried out with an AUTOLAB PGSTAT10 (ECOCHEMIE, Utrecht, The Netherlands) in a custombuilt three-electrode setup consisting of a platinum counter electrode, a mercurous sulfate reference electrode $\left(\mathrm{V}_{\mathrm{MSE}}=0.61 \mathrm{~V}_{\mathrm{NHE}}\right)$ and a gold working electrode (held by a screw cap to the bottom of the cell and exposing a geometric area of $0.44 \mathrm{~cm}^{2}$ to the electrolyte solution). Scans were carried out using a frequency range from $10 \mathrm{kHz}$ to $0.1 \mathrm{~Hz}$ in $1 \mathrm{mM} \mathrm{K}{ }_{3} \mathrm{Fe}(\mathrm{CN})_{6} / \mathrm{K}_{4} \mathrm{Fe}(\mathrm{CN})_{6}$ containing $0.1 \mathrm{M} \mathrm{K}_{2} \mathrm{SO}_{4}$ at $-0.2 \mathrm{~V}_{\mathrm{MSE}}$ with a $5 \mathrm{mV}$ amplitude. The charge-transfer resistance $\left(\mathrm{R}_{\mathrm{CT}}\right)$ of the monolayer on gold was determined from the Nyquist plot by fitting the experimental data to an equivalent Randles circuit containing a charge-transfer resistance of the monolayer in parallel with the capacitance of the monolayer and in series with the resistance of the solution. ${ }^{[45,46]}$

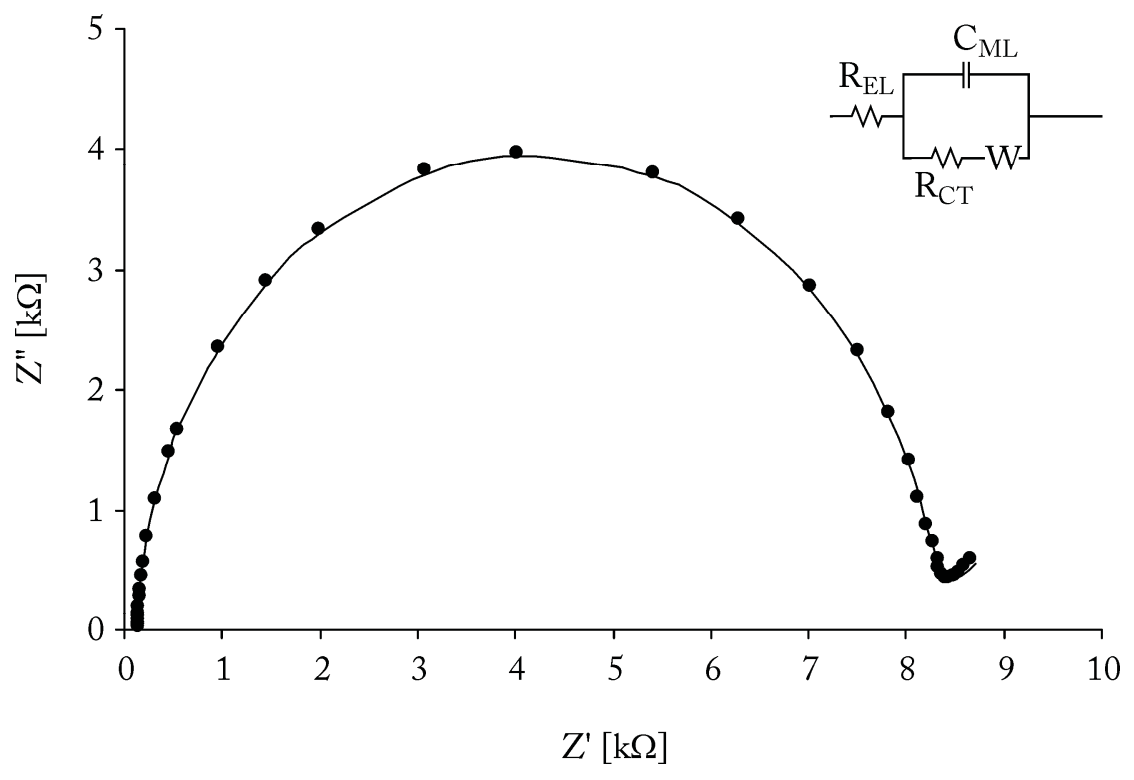

This figure is taken as an example of a Nyquist electrochemical impedance spectroscopy plot of a $\beta-\mathrm{CD}$ printboard after adsorption of guest $4 \mathrm{~b}$ from solution and the corresponding fit to an equivalent Randles circuit $\left(\mathrm{R}_{\mathrm{CT}}=8.1 \mathrm{k} \Omega\right)$.

NMR spectroscopy was carried out at $25{ }^{\circ} \mathrm{C}$ using a Varian Unity Inova 300 spectrometer and Varian Unity $400 \mathrm{WB}$ spectrometer respectively. ${ }^{1} \mathrm{H}$ NMR 
(300 MHz) and ${ }^{13} \mathrm{C}$ NMR $(100 \mathrm{MHz})$ chemical shifts are given relative to residual $\mathrm{CHCl}_{3}$ (7.27 ppm and $77.0 \mathrm{ppm}$, respectively).

FAB mass spectrometry was carried out with a Finnigan MAT90 spectrometer with $m$-nitrobenzylalcohol (NBA) as the matrix.

\subsection{References and notes}

[1] Y. Xia, G. M. Whitesides, Angew. Chem. Int. Ed. 1998, 37, 550; Angerw. Chem. 1998, 110, 568.

[2] B. Michel, A. Bernard, A. Bietsch, E. Delamarche, M. Geissler, D. Juncker, H. Kind, J.-P. Renault, H. Rothuizen, H. Schmid, P. Schmid-Winkel, R. Stutz, H. Wolf, IBMJ. Res. Dev. 2001, 45, 697.

[3] S. Krämer, R. R. Fuierer, C. B. Gorman, Chem. Rev. 2003, 103, 4367.

[4] A. Bernard, J.-P. Renault, B. Michel, H. R. Bosshard, E. Delamarche, Adv. Mater. 2000, 12, 1067.

[5] L. Yan, W. T. S. Huck, X.-M. Zhao, G. M. Whitesides, Langmuir 1999, 15, 1208.

[6] M. Jaschke, H.-J. Butt, Langmuir 1995, 11, 1061.

[7] (a) R. D. Piner, J. Zhu, F. Xu, S. Hong, C. A. Mirkin, Science 1999, 283, 661.

(b) C. A. Mirkin, S. Hong, R. D. Levine, ChemPhysChem 2001, 2, 37.

[8] L. M. Demers, D. S. Ginger, S.-J. Park, Z. Li, S.-W. Chung, C. A. Mirkin, Science 2002, 296, 1836.

[9] J. Huskens, M. A. Deij, D. N. Reinhoudt, Angerw. Chem. Int. Ed. 2002, 41, 4467; Angerw. Chem. 2002, 114, 4647.

[10] J. Szejtli, in Comprehensive Supramolecular Chemistry, Vol. 3 Cyclodextrins (Vol. Eds. J. Szejtli, T. Osa), Pergamon Press, Oxford, 1996. pp. 5-41 and pp. 189-205.

[11] M. V. Rekharsky, Y. Inoue, Chem. Rev. 1998, 98, 1875.

[12] H. Schönherr, M. W. J. Beulen, J. Bügler, J. Huskens, F. C. J. M. Van Veggel, D. N. Reinhoudt, G. J. Vancso, J. Am. Chem. Soc. 2000, 122, 4963.

[13] M. W. J. Beulen, J. Bügler, M. R. de Jong, B. Lammerink, J. Huskens, H. Schönherr, G. J. Vancso, A. Boukamp, H. Wieder, A. Offenhäuser, W. Knoll, F. C. J. M. Van Veggel, D. N. Reinhoudt, Chem. Eur. J. 2000, 6, 1176.

[14] A. Ulman, An Introduction to Ultrathin Organic Films, Academic Press, San Diego, 1991.

[15] M. R. de Jong, J. Huskens, D. N. Reinhoudt, Chem. Eur. J. 2001, 7, 4164. 
[16] Generally, a molecular printboard is a SAM of receptor molecules that have specific recognition sites, for example, molecular cavities, at which one can immobilize molecules from aqueous solutions through specific and directional supramolecular interactions.

[17] See guest 3 (Scheme 3.1). This calix[4]arene derivative has guanidinium functionalities at the upper rim to increase water solubility. Poly(ethylene glycol) (PEG) linkers at the lower rim space out the two adamantyl functionalities in order allow this molecule to form inclusion complexes with two neighboring $\beta$-CDs on the $\beta$-CD SAM (lattice constant $\beta$-CD $\sim 1.8 \mathrm{~nm}$ ), while retaining water solubility and preventing nonspecific interactions with the printboard.

[18] A. Mulder, T. Auletta, A. Sartori, S. Del Ciotto, A. Casnati, R. Ungaro, J. Huskens, D. N. Reinhoudt, J. Am. Chem. Soc. 2004, 126, 6627.

[19] J. Huskens, A. Mulder, T. Auletta, C. A. Nijhuis, M. J. W. Ludden, D. N. Reinhoudt, J. Am. Chem. Soc. 2004, 126, 6784.

[20] O. Crespo-Biel, M. Péter, C. M. Bruinink, B. J. Ravoo, D. N. Reinhoudt, J. Huskens, Chem. Eur. J. 2005, 11, 2426.

[21] C. A. Nijhuis, J. Huskens, D. N. Reinhoudt, J. Am. Chem. Soc. 2004, 126, 12266.

[22] T. Auletta, B. Dordi, A. Mulder, A. Sartori, S. Onclin, C. M. Bruinink, M. Péter, C. A. Nijhuis, H. Beijleveld, H. Schönherr, G. J. Vancso, A. Casnati, R. Ungaro, B. J. Ravoo, J. Huskens, D. N. Reinhoudt, Angerw. Chem. Int. Ed. 2004, 43, 369; Angerw. Chem. 2004, 116, 373.

[23] M. W. J. Beulen, J. Bügler, B. Lammerink, F. A. J. Geurts, E. M. E. F. Biemond, K. G. C. Van Leerdam, F. C. J. M. Van Veggel, J. F. J. Engbersen, D. N. Reinhoudt, Langmuir 1998, 14, 6424.

[24] A. Benninghoven, Angew. Chem. Int. Ed. 1994, 33, 1023; Angew. Chem. 1994, 106, 1075.

[25] A drawback of surface modification of PDMS, including UVO treatment for long periods of time (> $30 \mathrm{~min})$, is that the formation of a thin silica-like surface layer $\left(\mathrm{SiO}_{\mathrm{x}}\right)$ changes the mechanical and adhesive properties of the stamp. The resulting change in deformation characteristics of the PDMS stamp under slight pressure, in order to make conformal contact between the stamp and the substrate, is the most probable cause that affects accurate pattern reproduction of the original stamp features (10 $\mu \mathrm{m}$ with $5 \mu \mathrm{m}$ spacing). See the printing results of line patterns (about $9 \mu \mathrm{m}$ with $6 \mu \mathrm{m}$ spacing, Figs. 3.1 and 3.2).

[26] J. Rao, J. Lahiri, L. Isaacs, R. M. Weis, G. M. Whitesides, Science 1998, 280, 708.

[27] Use of the per- $\beta-C D$ complex of $4 b$ is necessary to increase its water solubility (conditions: excess of native $\beta-\mathrm{CD}$ in water at $\mathrm{pH} 2$ ) in order to obtain a sufficient concentration of the ink solutions for $\mu \mathrm{CP}$ and $\mathrm{DPN}$ on the molecular printboard.

[28] J. J. Michels, M. W. P. L. Baars, E. W. Meijer, J. Huskens, D. N. Reinhoudt, J. Chem. Soc., Perkin Trans. 2 2000, 1914. 
on molecular printboards

[29] J. J. Michels, J. Huskens, D. N. Reinhoudt, J. Chem. Soc., Perkin Trans. 2 2002, 102.

[30] G. O. Mallory, J. B. Hajdu, Electroless Plating: Fundamentals and Applications, American Electroplaters and Surface Finishers Society, Orlando, FL, 1990.

[31] A. M. Bittner, X. C. Wu, K. Kern, Adv. Funct. Mater. 2002, 12, 432.

[32] X. C. Wu, A. M. Bittner, K. Kern, Langmuir 2002, 18, 4984.

[33] Relative humidity was found to play an important role in the present DPN experiments, since all successful writing experiments were carried out at humidities above $40 \%$. These results support the currently accepted view that relative humidity is essential to the success of DPN. See: S. Rozhok, R. Piner, C. A. Mirkin, J. Phys. Chem. B 2003, 107, 751.

[34] G. H. Degenhart, B. Dordi, H. Schönherr, G. J. Vancso, Langmuir 2004, 20, 6216.

[35] H. Ron, I. Rubinstein, Langmuir 1994, 10, 4566.

[36] H. Schmid, B. Michel, Macromolecules 2000, 33, 3042.

[37] K. Efimenko, W. E. Wallace, J. Genzer, J. Colloid Interface Sci. 2002, 254, 306.

[38] M. J. Owen, P. J. Smith, J. Adhes. Sci. Technol. 1994, 8, 1063.

[39] S. Perutz, J. Wang, E. J. Kramer, C. K. Ober, K. Elles, Macromolecules 1998, 31, 4272.

[40] S. K. Thanawala, M. J. Chaudhury, Langmuir 2000, 16, 1256.

[41] (a) H. Hillborg, U. W. Gedde, Polymer 1998, 39, 1991. (b) H. Hillborg, J. F. Ankner, U. W. Gedde, G. D. Smith, H. K. Yasuda, K. Wikström, Polymer 2000, 41, 6851.

[42] For supramolecular $\mu \mathrm{CP}$ experiments, the intrinsic hydrophobic character of PDMS $\left(\theta_{\mathrm{adv}}=120^{\circ}\right)$ is insufficient to assure transfer of polar guest molecules from the PDMS stamp onto the $\beta-\mathrm{CD}$ printboard.

[43] H. Hillborg, N. Tomczak, A. Olàh, H. Schönherr, G. J. Vancso, Langmuir 2004, $20,785$.

[44] C. Donzel, M. Geissler, A. Bernard, H. Wolf, B. Michel, J. Hilborn, E. Delamarche, Adv. Mater. 2001, 13, 1164.

[45] (a) B. A. Boukamp, Solid State Ionics 1985, 18-19, 136. (b) B. A. Boukamp, Solid State Ionics 1986, 20, 31 .

[46] This circuit is a leaking capacitor that consists of a charge-transfer resistance of the monolayer (RCT) parallel with the capacitance of the monolayer (CML), and in series with the resistance of the solution/electrolyte (REL). A Warburg element (W) in series with RCT describes the diffusion of the redox couple from and to the electrolyte. 


\section{Stamps for high-resolution soft lithography"}

This chapter describes a novel soft-lithographic technique by combining capillary force lithography (CFL) and replica molding (REM) for the fabrication of high-resolution, second-generation stamps for soft lithography. The fabrication of different gold patterns at a resolution of $\sim 125 \mathrm{~nm}$ by application of these stamps in several microcontact printing $(\mu C P)$ experiments illustrates the applicability of the technique for high-resolution soft lithography, without the need for cleanroom facilities.

" Parts of this chapter have been published in: C. M. Bruinink, M. Péter, M. J. de Boer, L. Kuipers, J. Huskens, D. N. Reinhoudt, Adv. Mater. 2004, 16, 1086 (Copyright (C) 2004 Wiley-VCH Verlag GmbH \& Co. KGaA); C. M. Bruinink, M. Péter, P. A. Maury, M. J. de Boer, L. Kuipers, J. Huskens, D. N. Reinhoudt, Adv. Funct. Mater. 2006, 16, 1555 (Copyright (C) 2006 Wiley-VCH Verlag GmbH \& Co. KGaA). 


\subsection{Introduction}

Photolithography has been the main technology for integrated circuit (IC) fabrication during the last decades, ${ }^{[1]}$ however, its extension to the sub-100 $\mathrm{nm}$ range requires the development of state-of-the-art lithographic techniques: e.g., deep-UV and extreme-UV photolithography, ${ }^{[2,3]}$ soft $\mathrm{X}$-ray lithography, ${ }^{[4]}$ electron-beam writing, ${ }^{[5]}$ and ion-beam lithography. ${ }^{[6]}$ The costs render these techniques less suitable for exploratory research applications, in which requirements such as pattern uniformity, reproducibility, and accurate alignment are not stringent. The development of complementary non-photolithographic techniques, including soft lithography, ${ }^{[7]}$ nanoimprint lithography (NIL), ${ }^{[8]}$ and capillary force lithography $(\mathrm{CFL})^{[9]}$ has been successful during the last decade in the field of micro- and nanofabrication.

Soft lithography ${ }^{[7]}$ is a versatile and cost-effective patterning technique for the routine fabrication of structures of $\geq 500 \mathrm{~nm}$. Soft lithography uses elastomeric poly(dimethylsiloxane) (PDMS) stamps to transfer a relief pattern to the surface of a substrate by conformal contact. Generally, high-resolution soft lithography still depends on the availability of state-of-the-art lithographic techniques for stamp fabrication. Several elegant and cost-effective methods for stamp fabrication with minimum feature sizes of about $200 \mathrm{~nm}$ have been reported by Whitesides. ${ }^{[10,11]}$ The basic strategy is to start from elastomeric PDMS stamps with micrometer-size features and exploit non-photolithographic techniques to fabricate submicrometer-size patterns. Several methods exploit the elastomeric properties of commercial PDMS: by microcontact printing $(\mu \mathrm{CP})$ with stamps under lateral compression, ${ }^{[12]}$ under uniaxial stretching in the plane of the stamp or under vertical pressure normal to the plane of the stamp; ${ }^{[10]}$ or by replica molding (REM) against PDMS stamps under lateral compression. ${ }^{[13]}$ Of a greater impact are alternatives like lithographic molding ${ }^{[14]}$ and repetitive REM that combine the facile fabrication of robust high-resolution masters with that of second-generation stamps.

One technique that also has the potential to fabricate these second-generation masters is capillary force lithography (CFL).$^{[9,15]} \mathrm{CFL}$ is an emerging nonphotolithographic technique that combines the essential feature of $\mathrm{NIL}^{[8]}$ - molding a polymer melt - with the key element of soft lithography ${ }^{[7]}$ - molding with an 
elastomeric stamp for large-area patterning of polymer films. At a temperature (T) above the glass-transition temperature $\left(\mathrm{T}_{\mathrm{g}}\right)$ of the polymer, capillary forces drive the polymer to conform to the mold pattern. The initial polymer film thickness and the feature dimensions of the mold determine the type of the resulting polymer patterns. CFL of thick polymer layers result in a negative copy of the mold, with a residual layer that remains on the substrate. Upon decreasing the initial polymer film thickness, the (concave) meniscus cannot rise to completely fill the spaces between the mold and the substrate, owing to polymer insufficiency, resulting in the formation of high-resolution polymer structures at the feature edges of the original stamp patterns. To the best of our knowledge, this chapter describes the first practical application of these CFL polymer structures as potential masters for high-resolution soft lithography. Structural analysis was carried out by atomic force microscopy (AFM) and scanning electron microscopy (SEM) to reveal information on the mechanical stability of the CFL polymer templates during stamp processing and the pattern transfer fidelity by application of the resulting stamps in soft lithography.

\subsection{Results and discussion}

\subsubsection{General procedure for the fabrication of high-resolution polymer templates}

Scheme 4.1 depicts the basis for the present soft-lithographic procedure for generating high-resolution polymer templates on substrates by CFL of thermoplastic polymers.

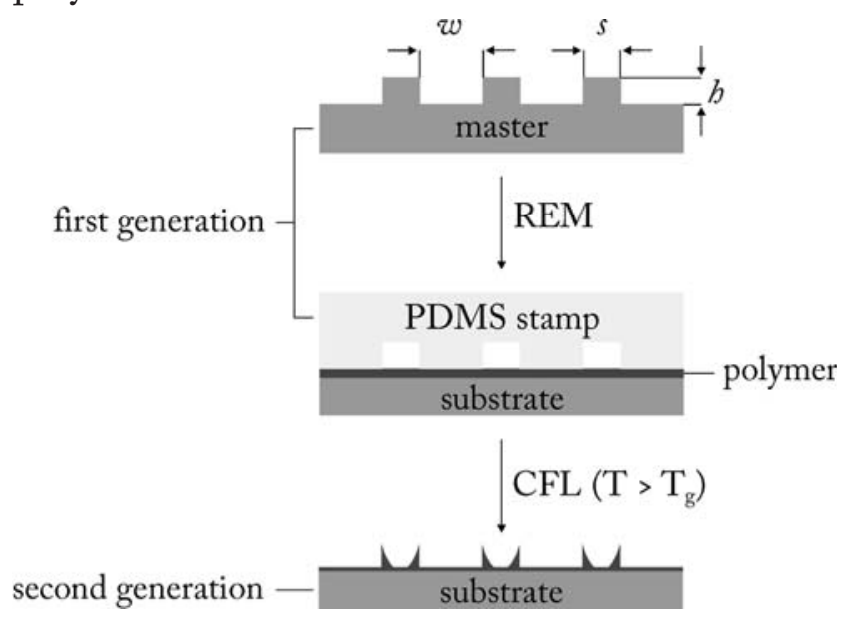

Scheme 4.1 Schematic representation of the general (two-stage) fabrication procedure of second-generation polymer templates: (1) replica molding (REM) of the first-generation master into PDMS stamps $(w=5 \mu \mathrm{m}, s=3 \mu \mathrm{m}, h=1.2 \mu \mathrm{m}) ;(2) \mathrm{CFL}^{[9]}$ on thin polymer layers at $\mathrm{T}>\mathrm{T}_{\mathrm{g}}$ of the polymer for several hours. 
A liquid prepolymer of PDMS (Sylgard 184, Dow Corning Corporation) was cast against a silicon master. All the masters in this study were fabricated by standard photolithography and contain either continuous (lines) or discontinuous (pillars) features of micrometer size (width, $w=5 \mu \mathrm{m}$, spacing, $s=3 \mu \mathrm{m}$, height, $h=1.2 \mu \mathrm{m}$ ). The PDMS was cured for $1-18 \mathrm{~h}$ at $60^{\circ} \mathrm{C}$ in order to obtain stamps of minimal $\left(\sim 0.5 \mathrm{~N} \cdot \mathrm{mm}^{-2}\right)$ to medium modulus $\left(\sim 2 \mathrm{~N} \cdot \mathrm{mm}^{-2}\right),{ }^{[16]}$ respectively, suitable for conducting CFL over large areas. Special care was taken to prevent the interference of dust particles by bringing the substrate and stamp into contact with minimal delay. Larger dust particles in particular were found to act as sites at which conformal contact was gradually lost during CFL. High-temperature processing is known to limit the performance of PDMS stamps. ${ }^{[9]}$ Without taking proper precautions, the contact between the substrates and PDMS stamps cannot withstand the thermal stress that builds up owing to differences in thermal expansion coefficients ( $\alpha_{\text {PDMS }}$ " $\left.\alpha_{S_{\mathrm{S}}}\right)$. To prevent separation, the thickness of the PDMS stamps was kept down to about $1 \mathrm{~mm}$, in order to ensure sufficient flexibility. ${ }^{[17]}$ This measure was found to improve the overall performance of commercial PDMS for CFL processing of polymer layers effectively, even though the stamp gradually hardens by thermal curing during processing.

To obtain high-resolution polymer templates, thin layers $(\sim 35-200 \mathrm{~nm})$ of thermoplastic polymers, e.g., poly(methylmethacrylate) (PMMA) and polystyrene (PS) were applied onto silicon substrates by spin coating. At this range of polymer film thicknesses, no dewetting effects by physical confinement - which could impair the alignment of the polymer features at the feature edges of the PDMS stamp - were to be expected. ${ }^{[18]}$ The initial conditions for CFL on thin polymer layers are crucial for the formation of useful symmetrical template structures. Of all the different procedures, pre-equilibration of the PDMS stamp and the substrate to the annealing temperature before bringing the PDMS stamp into conformal contact with the substrate was found to result in the most-symmetrical polymer structures over large areas. The annealing temperature $\mathrm{T}$ was set sufficiently above the glass-transition temperature $\mathrm{T}_{\mathrm{g}}\left(\mathrm{T} \approx \mathrm{T}_{\mathrm{g}}+40^{\circ} \mathrm{C}\right)$ in order to ensure polymer mobility for patterning the polymer layers during CFL. Typical annealing times were chosen in the range of 2-20 h. It was found by optical inspection that cooling down the stamp and substrate 
for $5 \mathrm{~s}$ (instead of $30 \mathrm{~min}$ ) at room temperature was already sufficient to release the stamp from the substrate, without generating (microscopic) distortions to the polymer template.

Capillary kinetics of a polymer melt in permeable microchannels ${ }^{[19]}$ have been shown to depend on several parameters, such as time, polymer flow (temperature, type of the polymer, wetting capabilities of the stamp) and the amount of polymer accessible to fill the spaces between the mold and the substrate (initial polymer film thickness, fill factor of the stamp). As a result, one can expect to have control over the lateral and vertical dimensions of the resultant polymer features by changing any of these parameters (Fig. 4.1).
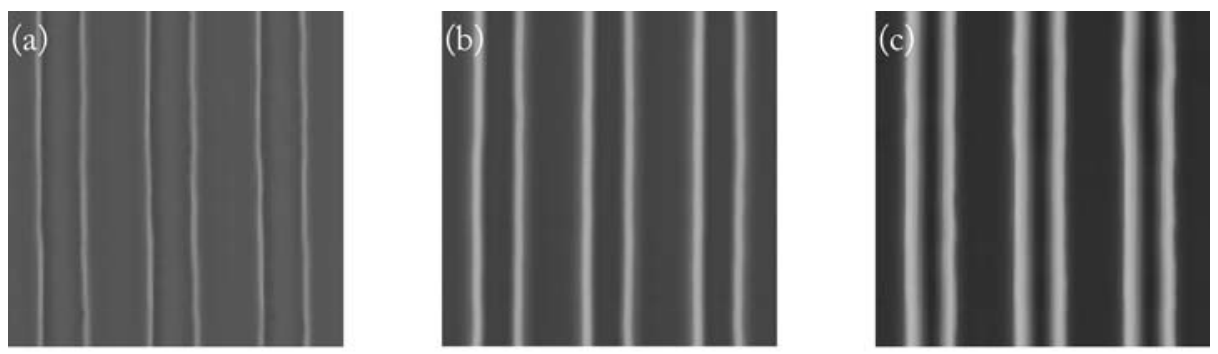

(d)
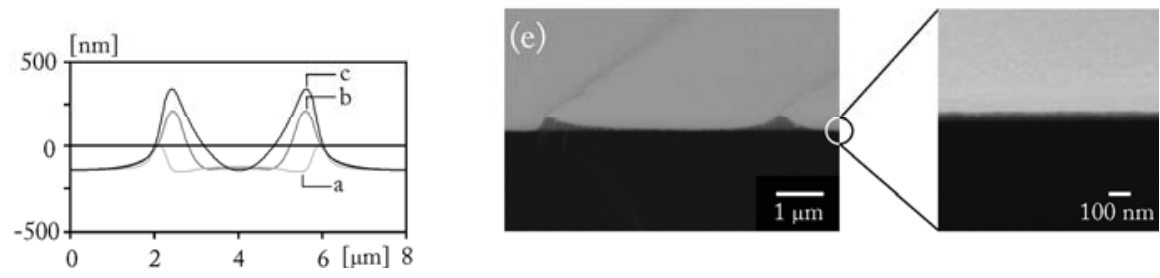

Figure 4.1 Contact-mode (CM) AFM height images ( $\mathrm{z}$ range: $1000 \mathrm{~nm}$; image size: $25 \mu \mathrm{m} \times 25 \mu \mathrm{m})$ of different high-resolution PS templates. These templates were fabricated by CFL on initial polymer thicknesses of $35 \mathrm{~nm}$ (a), $80 \mathrm{~nm}$ (b), and $150 \mathrm{~nm}$ (c) at $150{ }^{\circ} \mathrm{C}$ for $20 \mathrm{~h}$. The feature widths (full width at half maximum, FWHM) of these PS structures, respectively $440 \mathrm{~nm}, 680 \mathrm{~nm}$, and $850 \mathrm{~nm}$, were determined from the average height cross-sectional analysis of the respective meniscus of each template (d). (e) SEM image of the crosssectional view of a symmetrical PS template on silicon. From the close-up SEM image, a thin residual layer is seen on the silicon substrate.

Fig. 4.1 shows the result of increasing the initial polymer film thickness from $35 \mathrm{~nm}$ to $150 \mathrm{~nm}$ with the other process parameters kept constant (PS, $M_{w}=240 \mathrm{kDa}$, $150{ }^{\circ} \mathrm{C}, 20 \mathrm{~h}$ ). Cross-sectional AFM analyses of the resulting PS templates (Fig. 4.1d) reveal that it is possible to exert certain control over the feature line width of the 
polymer template, ranging in this case from $440 \mathrm{~nm}$ to $850 \mathrm{~nm}$ (Figs. 4.1a-c). Adjusting the other process parameters should result in polymer templates with an even larger range of feature widths. Under the present process conditions, a thin residual layer of $<100 \mathrm{~nm}$ is always present in the template, protecting the underlying substrate from direct post-processing (Fig. 4.1e).

\subsubsection{Fabrication of second-generation PDMS stamps}

These CFL polymer templates of thermoplastic polymers are directly applicable in the stamp processing stage by replica molding (REM) (Fig. 4.2a). The only requirement for thermoplastic polymer templates in order to function as robust masters for the fabrication of second-generation stamps is to have a $T_{g}$ that is several tens of degrees higher than the thermal curing temperature of PDMS $\left(\mathrm{T}=60{ }^{\circ} \mathrm{C}\right)$. The cross-sectional AFM analysis of a representative CFL master (in this case PMMA, $M_{w}=38 \mathrm{kDa}$ ) illustrates the excellent uniformity of the polymer structures, on which the positions of the $440-n m$-high PMMA structures (FWHM is $\sim 680 \pm 100 \mathrm{~nm}$ ) correlate with the periodicity of the PDMS stamp (Fig. 4.2b).

(a)
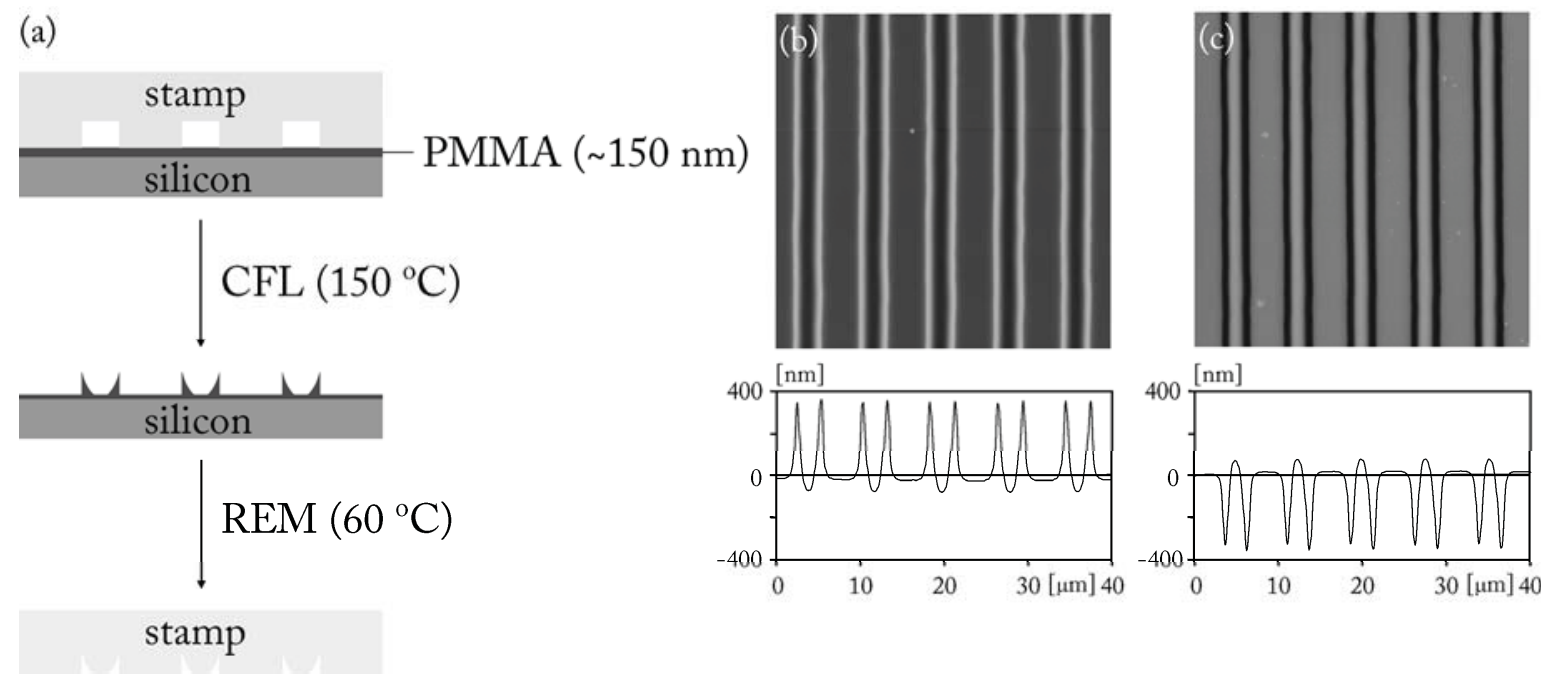

Figure 4.2 (a) Schematic representation of the procedure for the fabrication of second-generation stamps using CFL polymer templates as masters. CM AFM height images (z range $800 \mathrm{~nm}$ ) of the high-resolution secondgeneration master (b) and the corresponding second-generation PDMS stamp (c) (image size $50 \mu \mathrm{m} \times 50 \mu \mathrm{m}$ ), including the corresponding cross-sectional analysis. The PMMA masters were fabricated by CFL with flexible PDMS stamps on a $150 \mathrm{~nm}$ PMMA layer at $150{ }^{\circ} \mathrm{C}$ for $6 \mathrm{~h}$. Second-generation PDMS stamps were fabricated by casting prepolymer PDMS against the PMMA master and curing for $1 \mathrm{~h}$ at $60{ }^{\circ} \mathrm{C}$. Post-curing of the second-generation stamps was done for another $18 \mathrm{~h}$ at $60^{\circ} \mathrm{C}$ after release from the master. 
The general procedure (Section 4.2.1) is, therefore, ideal for the fabrication of second-generation masters using thermoplastic polymers. The remaining irregularities in the width and height of the PMMA edge patterns present in the master most probably originate from dust particles interfering with the alignment of the stamp to the substrate since CFL was carried out outside the cleanroom environment.

The fabrication of second-generation PDMS from CFL templates was done using the same REM procedure for the generation of the first-generation PDMS stamps. Fig. 4.2c shows an AFM image of the resulting PDMS stamp. The corresponding cross-sectional AFM analysis confirms that REM of second-generation PMMA masters was achieved with high fidelity, as shown by the $\sim 420 \mathrm{~nm}$ deep indentation (FWHM is $~ 950 \pm 100 \mathrm{~nm}$ ) in the PDMS stamps. The low interfacial free energy, the elasticity of PDMS, and its chemical inertness to PMMA allow the generation of multiple copies of the PMMA master into second-generation PDMS stamps. No noticeable degradation or damage of the PMMA master was observed after fabrication of more than ten copies.

\subsubsection{Application of second-generation stamps in soft lithography and the selective etching of gold}

To illustrate the applicability in soft lithography, the second-generation PDMS stamps were used in a series of microcontact printing $(\mu \mathrm{CP})$ experiments. As part of soft lithography, $\mu \mathrm{CP}$ is a non-photolithographic patterning technique for the facile formation of patterns of self-assembled monolayers (SAMs) of alkanethiols on surfaces of coinage metals (e.g., gold, silver, palladium, copper) upon conformal contact of an ink-containing elastomeric PDMS stamp with the metal surface. ${ }^{[7]}$ Additionally, the resulting SAM patterns are known to comprise of a class of efficient ultrathin resists in the selective wet etching of the underlying metal layer. The entire $\mu \mathrm{CP}$ process for the routine fabrication of metal patterns on silicon surfaces is shown in Scheme 4.2. 


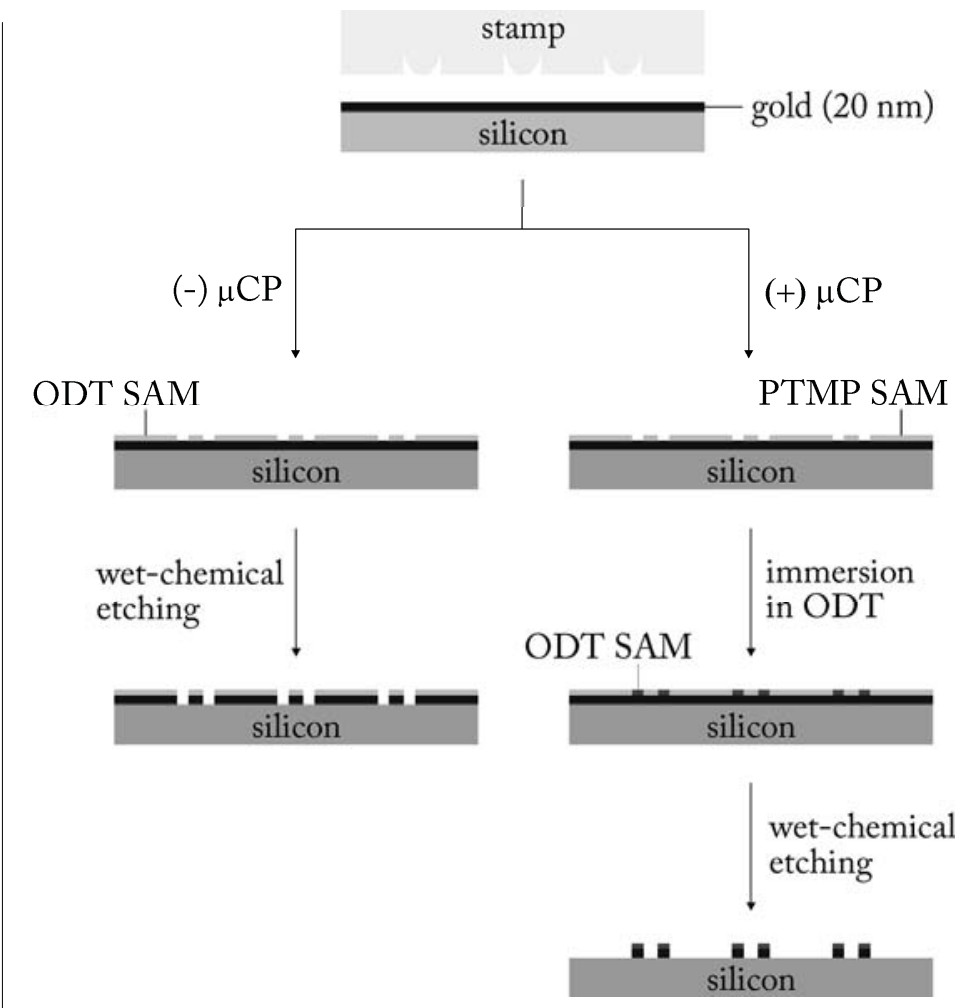

Scheme 4.2 Schematic representation of two different $\mu \mathrm{CP}$ processes using second-generation stamps to fabricate gold features on silicon. (-) $\mu \mathrm{CP}$ includes printing with octadecanethiol (ODT) (left). (+) $\mu \mathrm{CP}$ includes printing with pentaerythritol-tetrakis(3-mercaptopropionate) (PTMP) and subsequent protection of the bare gold by dipping the in ODT solution (right). Selective wet-chemical etching of the gold results in trenches in gold (in case of $(-) \mu \mathrm{CP}$ ) and gold lines (in case of $(+) \mu \mathrm{CP}$ ).

Many selective etch solutions are known for the wet-chemical etching of different coinage metals using SAMs as etch resists. ${ }^{[7]}$ For gold, the most selective etch solution comprises a basic solution containing $\mathrm{K}_{2} \mathrm{~S}_{2} \mathrm{O}_{3} / \mathrm{K}_{3} \mathrm{Fe}(\mathrm{CN})_{6} / \mathrm{K}_{4} \mathrm{Fe}(\mathrm{CN})_{6}$ at $\mathrm{pH}$ 14. ${ }^{[20]}$ This aqueous etch solution is not only less hazardous, but also superior to the conventional gold etch solution (aqueous $\mathrm{KCN}$ at $\mathrm{pH} 14$ ) in terms of etch rate and selectivity (i.e. defects and line-edge resolution). The function of the different components of this etch solution during the etching of gold has been shown in an extensive study. ${ }^{[20]}$ The AFM images in Fig. 4.3 illustrate a higher degree of definition of the gold features with time. From the etching plot (Fig. 4.3d) it is clear that the gold etching in this solution is monotonous in time, representing an etch rate of $3.5 \mathrm{~nm} \cdot \mathrm{min}^{-1}$. The origin for the apparent offset in etching $(\sim 30 \mathrm{~s})$ is unknown. 

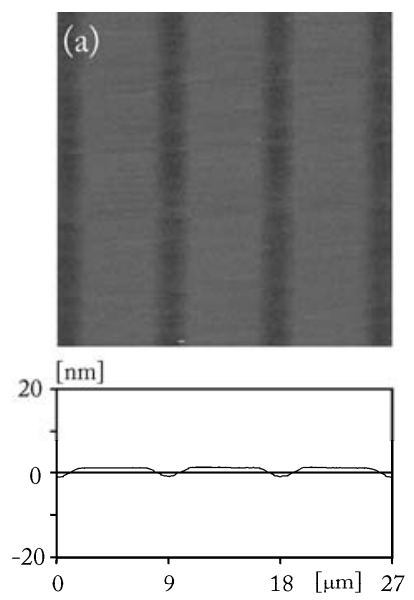

(d)
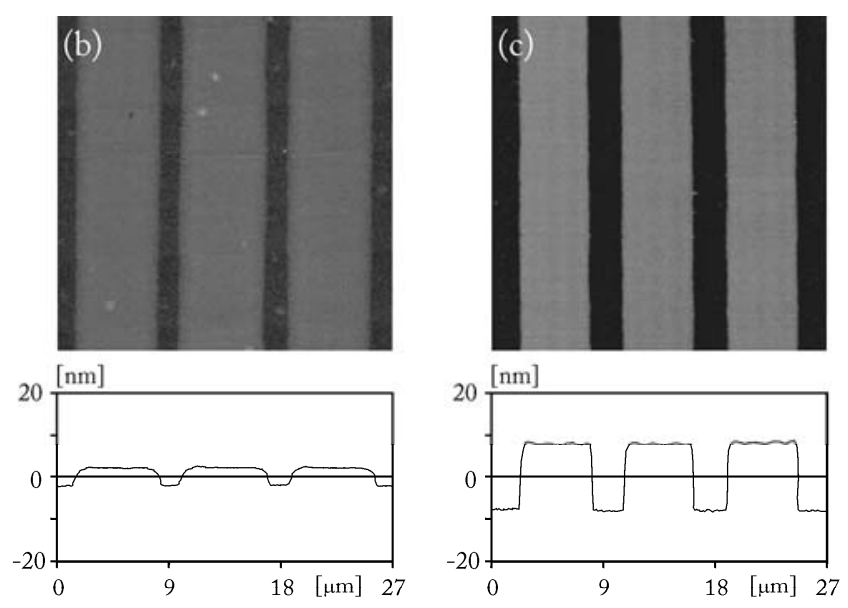

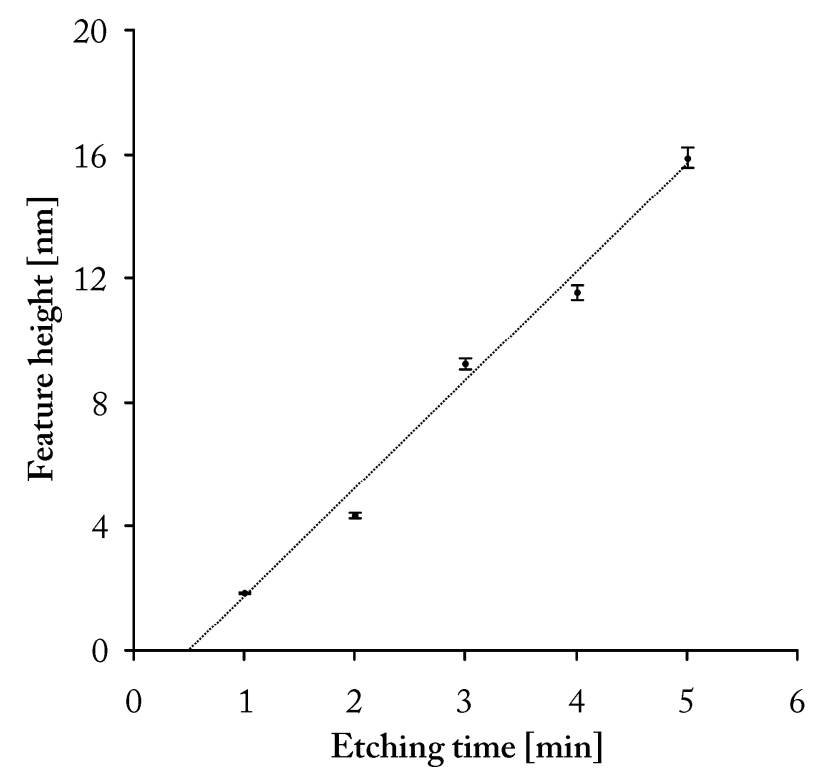

Figure 4.3 CM AFM height images ( $\mathrm{z}$ range: $40 \mathrm{~nm}$; image size: $27 \mu \mathrm{m} \times 27 \mu \mathrm{m}$ ) of the progression of gold etching using ODT SAMs as etch resists, including the average height cross-sectional analyses at etch times of $1 \mathrm{~min}(\mathrm{a}), 2 \mathrm{~min}$ (b), and $5 \mathrm{~min}$ (c). (d) Plot of the feature height versus the etch time of gold in the etch solution at room temperature.

The experimental time for complete etching of a $20 \mathrm{~nm}$-thick gold layer $\left(t_{1}=6-6.5 \mathrm{~min}\right.$, including the offset) is close to the literature value $\left(t_{1}=7-8 \mathrm{~min}\right),{ }^{[20]}$ and therefore the total etch time was set to $7 \mathrm{~min}(=20 \%$ overetching with respect to the theoretical etch time) to ensure complete and reproducible etching of the bare gold regions. Overetching of the gold layer is possible, as the etch window, i.e. the additional time of protection of the gold by SAMs, of this etch solution is known to take several minutes (depending on the type of SAM). ${ }^{[20]}$ Fig. 4.4 illustrates different 
gold features by application of the second-generation PDMS stamps in several $\mu \mathrm{CP}$ processes.
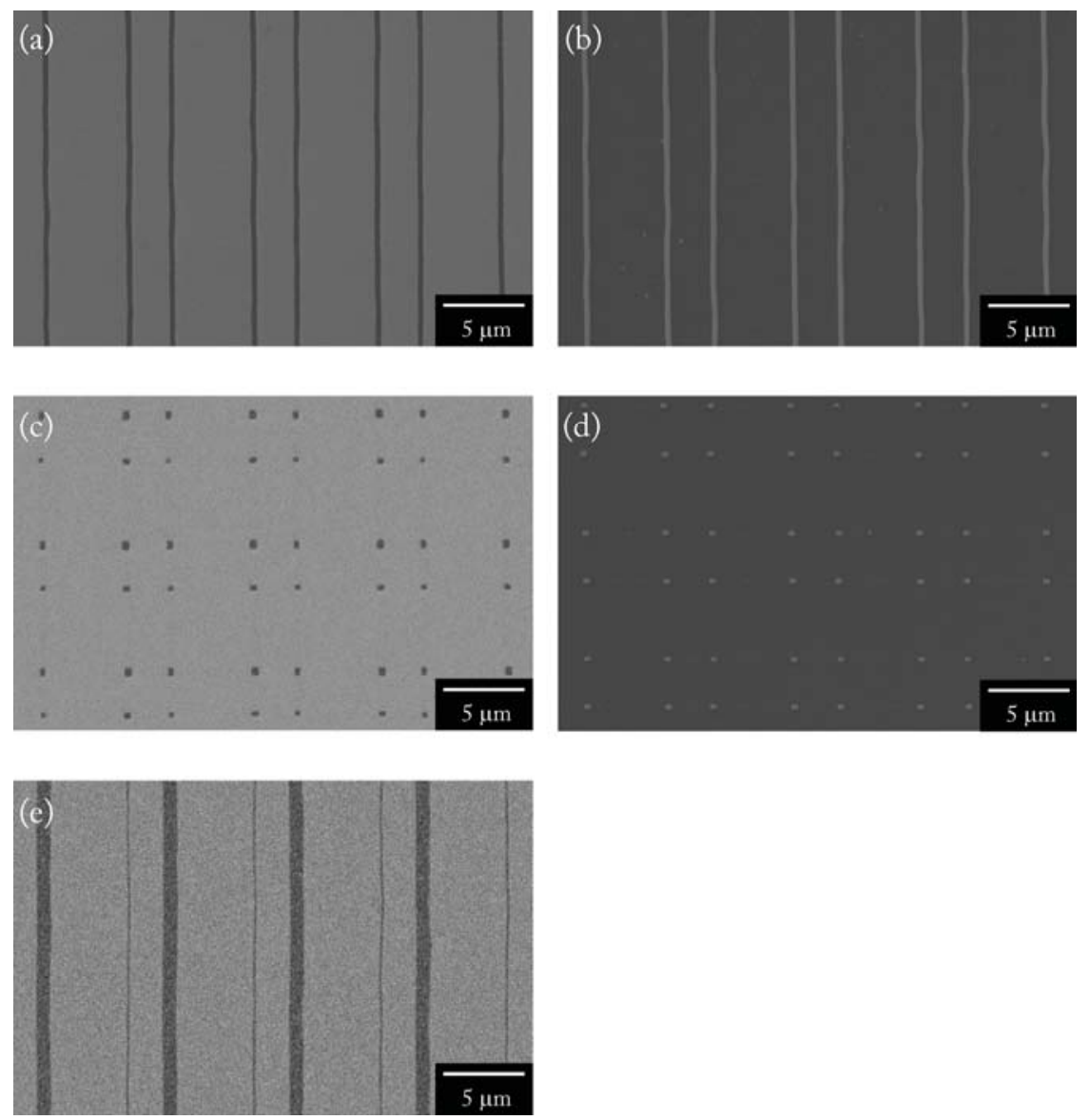

Figure 4.4 SEM images of different structures in gold on silicon, resulting from several $\mu \mathrm{CP}$ experiments with second-generation PDMS stamps and subsequent wet-chemical etching of the gold. (a) Arrays of $\sim 350 \mathrm{~nm}$-wide trenches in gold by $(-) \mu \mathrm{CP}$ with ODT for $60 \mathrm{~s}$ and subsequent selective wet-chemical etching of gold for $7 \mathrm{~min}$. (b) Arrays of $\sim 350 \mathrm{~nm}$-wide gold lines by $(+) \mu \mathrm{CP}$ with PTMP for $60 \mathrm{~s}$, dipping in an ODT solution for $5 \mathrm{~s}$ and subsequent selective wet-chemical etching of gold for $10 \mathrm{~min}$. (c/d) 350 nm-wide hole/dot grids of gold on silicon by cross $-\mu \mathrm{CP}$, i.e. two sequential printing steps on the same substrate with $90^{\circ}$ rotation between the prints. The printing time in this particular experiment was $30 \mathrm{~s}$ for each printing step ( $1 \mathrm{~min}$ in total). (e) Arrays of alternating $\sim 125 \mathrm{~nm}$-wide and $\sim 800 \mathrm{~nm}$-wide trenches in gold by (-) $\mu \mathrm{CP}$ (same conditions) with a secondgeneration PDMS stamp containing asymmetric features.

Arrays of $\sim 350 \mathrm{~nm}$-wide trenches in gold layers (Fig. 4.4a) were fabricated by $\mu \mathrm{CP}$ of octadecanethiol (ODT) on a $20 \mathrm{~nm}$-thick gold layer and subsequent selective wetchemical etching. Since only the edges of the original (first-generation) master pattern are transferred into the gold layer, the present $\mathrm{CFL}$ technique to fabricate secondgeneration stamps in combination with $\mu \mathrm{CP}$ and wet-chemical etching is a new 
alternative to the general class of edge-lithographic techniques. ${ }^{[21,22,23,24]}$ The major advantage of this technique over the existing edge-lithographic techniques is that it does not require any cleanroom facilities for the fabrication of the structures.

The discrepancy between the lateral dimensions of the trenches in gold $(\sim 350 \mathrm{~nm})$ and the indentations present on the second-generation PDMS stamp (Fig. 4.2c, crosssectional analysis FWHM $\sim 950 \mathrm{~nm}$ ) suggests that diffusion ${ }^{[25]}$ and deformation of the PDMS stamp ${ }^{[26]}$ during printing contribute to the reduction of the feature dimensions. The inverse structures, arrays of $\sim 350 \mathrm{~nm}$-wide gold lines, were fabricated by positive microcontact printing $((+) \mu \mathrm{CP})^{[27]}$ with PDMS stamps from the same second-generation master (Fig. 4.4b). (+) $\mu \mathrm{CP}$ has been recently developed by IBM to invert the final pattern in gold without the need for new masters ${ }^{[27]}$ and employs printing with pentaerythritol-tetrakis(3-mercaptopropionate) (PTMP), which is compatible with the requirements of $\mu \mathrm{CP}$, but incapable of forming etch-blocking SAMs. Adsorption of octadecanethiol (ODT) from solution to the bare gold areas and subsequent wet-chemical etching yields the positive pattern. It was found that PTMP SAMs retard the etching of gold and longer etching $(+40 \%$ in terms of optimal etch time) was necessary to obtain good-quality patterns.

Cross-printing is a convenient approach to reduce the second lateral feature dimension, ${ }^{[10]}$ and accordingly to increase the inherent low pattern density of this edge lithographic technique. Figs. $4.4 \mathrm{c}$ and $4.4 \mathrm{~d}$ illustrate the resulting gold patterns by $90^{\circ}$ cross-printing with the same stamp prior to wet-chemical etching of the gold.

Although the ultimate resolution of the present technique was not the aim of this study, patterns of alternating $\sim 125 \mathrm{~nm}$ and $\sim 800 \mathrm{~nm}$-wide trenches in gold (Fig. 4.4e) were fabricated by printing with stamps containing very asymmetric features in order to illustrate the potential of printing sub-150 nm with second-generation stamps of commercial PDMS.

\subsubsection{Pattern transfer by reactive ion etching}

The resulting $20 \mathrm{~nm}$-thick gold patterns are sufficiently robust to serve as secondary etch masks in reactive ion etching (RIE) of the underlying silicon substrate, resulting in high aspect ratios. To achieve anisotropic etching of silicon, the black silicon method $(\mathrm{BSM})^{[28]}$ was applied on the etch apparatus prior to etching of the substrates. 
BSM is an experimental procedure that makes use of the fact that the parameter settings for anisotropic etching are close to the ones for the formation of black silicon (i.e. micro spikes) owing to micromasking. For the present RIE apparatus, black silicon was found on bare silicon wafers at the following parameter settings: $10 \mathrm{mTorr}$, $50 \mathrm{~W}, 40 \mathrm{sccm} \mathrm{SF} 6$ and $20 \mathrm{sccm} \mathrm{O}_{2}, 10{ }^{\circ} \mathrm{C}$. At this point, only minor changes to the parameter settings were necessary to obtain anisotropic profiles such as the one shown in Fig. 4.5 (anisotropy of $~ 0.85$ ). ${ }^{[29]}$

(a)

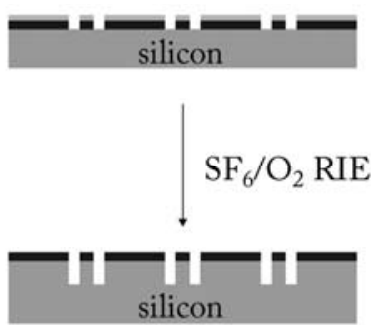

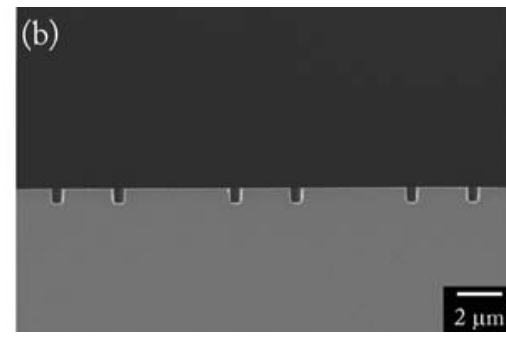

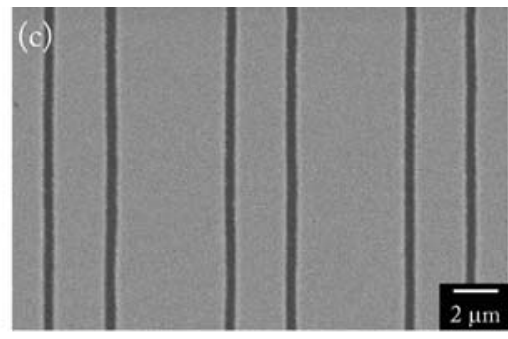

Figure 4.5 (a) Schematic representation of the RIE process. (b) High-resolution cross-sectional SEM image of $500 \mathrm{~nm}$-wide trenches after etching $650 \mathrm{~nm}$ into silicon using $\mathrm{SF}_{6} / \mathrm{O}_{2} \mathrm{RIE}$ (APC open, $50 \mathrm{~W}$ RF power, $40 \mathrm{sccm} \mathrm{SF}$, and $20 \mathrm{sccm} \mathrm{O}, 90 \mathrm{~s}$ ). A clear undercut on the order of $100 \mathrm{~nm}$ was found, resulting in a widening of the silicon trenches with respect to the lateral dimensions in the gold mask $(350 \mathrm{~nm})$. (c) Low-resolution SEM image of the same structures in top view.

Under these RIE conditions, the silicon etch rate was about $0.34 \mu \mathrm{m} / \mathrm{min}$ at a silicon over gold etch selectivity of more than 170 .

\subsection{Conclusions}

The present results in this chapter illustrate that the combination of capillary force lithography (CFL) and replica molding (REM) is a convenient and inexpensive approach for the fabrication of high-resolution, second-generation PDMS stamps from the corresponding second-generation masters without the need of state-of-theart lithographic techniques. The successful application of these stamps in $\mu \mathrm{CP}$ for the fabrication of $\sim 125 \mathrm{~nm}$ features in gold demonstrates its potential to extend the utility of soft lithography in general. Additionally, the general applicability of these templates in high-resolution nanofabrication procedures will allow fabrication of sub-100 nm features (see Chapter 5). 


\subsection{Experimental}

Chemicals and materials: All experiments were carried out in a regular chemical laboratory. Octadecanethiol (ODT), pentaerythritol-tetrakis(3-mercaptopropionate) (PTMP), ethanol, $\mathrm{NaOH}, \mathrm{Na}_{2} \mathrm{~S}_{2} \mathrm{O}_{3}, \mathrm{~K}_{3} \mathrm{Fe}(\mathrm{CN})_{6}$, and $\mathrm{K}_{4} \mathrm{Fe}(\mathrm{CN})_{6}$ were purchased from commercial resources in the highest available purity and used without further purification. A PDMS prepolymer kit (Sylgard 184 silicone elastomer) including PDMS curing agent (Sylgard silicone elastomer curing agent) was purchased from Dow Corning Corporation. Poly(methylmethacrylate) (PMMA, $\mathrm{M}_{\mathrm{w}}=38 \mathrm{kDa}$ and $350 \mathrm{kDa}$ ) and polystyrene (PS, $\mathrm{M}_{\mathrm{w}}=240 \mathrm{kDa}$ ) were purchased from Acros Organics. $100 \mathrm{~mm}$ p-type OSP silicon wafers were purchased from Okmetic and the $20 \mathrm{~nm}$ gold-on-silicon wafers from Ssens BV (Hengelo, The Netherlands).

Stamp fabrication: Stamps were fabricated by casting a 10:1 (v/v) mixture of PDMS and curing agent (Sylgard 184, Dow Corning) against a relief pattern of interest (i.e., silicon master or polymer template). After curing for $1 \mathrm{~h}$ at $60{ }^{\circ} \mathrm{C}$, the stamps were released at this curing temperature to avoid buildup of tension due to thermal shrinkage. ${ }^{[26 b]}$ Only the PDMS stamps for $\mu \mathrm{CP}$ were left in the oven at $60{ }^{\circ} \mathrm{C}$ for at least $18 \mathrm{~h}$ to ensure complete curing.

Capillary force lithography: Silicon substrates of $2 \mathrm{~cm} \times 2 \mathrm{~cm}$ were cleaned by ultrasonic treatment in acetone for $15 \mathrm{~min}$ and dried in a continuous stream of nitrogen. Polymer layers were applied onto these silicon substrates with minimal delay by spin coating a polymer solution in toluene at $3000 \mathrm{rpm}$ for $30 \mathrm{~s}$.

PDMS stamps for CFL were fabricated without additional post-curing in order to obtain very flexible stamps. The thickness of these PDMS stamps was about $1 \mathrm{~mm}$. In order to prevent separation, the PDMS stamps and the polymer layers were equilibrated to the annealing temperature for $30 \mathrm{~min}$ prior to bringing the stamp into contact with the polymer layer. The system was left at $40{ }^{\circ} \mathrm{C}$ above the $\mathrm{T}_{g}$ of the respective thermoplastic polymer for several hours. After cooling to ambient temperature (for $\sim 5 \mathrm{~s}$ ), the PDMS stamps were released from the substrates. The resulting polymer edge structures could function in this case as masters for the fabrication of second-generation PDMS stamps using the common molding and curing procedure. 
Microcontact printing: Cleaning of fresh gold substrates was performed by immersion into ethanol for $10 \mathrm{~min}$. The ink was applied to the second-generation PDMS stamps by exposing the stamps to a drop of ODT (0.3 mM) or PTMP $(1.8 \mathrm{mM})$ solution in ethanol for $1 \mathrm{~min}$. The gold substrates and stamps were dried with a continuous stream of nitrogen prior to printing. All the printing experiments were carried out manually with a print duration between $30-60 \mathrm{~s}$ in all cases. $\mathrm{Re}-$ inking of the stamp was only necessary in between printing steps for cross $-(+) \mu \mathrm{CP}$ experiments. To complete the $(+) \mu \mathrm{CP}$ procedure, the gold substrates were immersed into a $0.2 \mathrm{mM}$ solution of ODT for $5 \mathrm{~s}$.

Wet-chemical etching of gold: After $\mu \mathrm{CP}$, complete etching of the (bare) gold was achieved by immersing the gold substrates in an aqueous solution of $1.0 \mathrm{M} \mathrm{NaOH}$, $0.1 \mathrm{M} \mathrm{Na}_{2} \mathrm{~S}_{2} \mathrm{O}_{3}, 0.01 \mathrm{M} \mathrm{K}_{3} \mathrm{Fe}(\mathrm{CN})_{6}$, and $0.001 \mathrm{M} \mathrm{K}_{4} \mathrm{Fe}(\mathrm{CN})_{6}$ at room temperature for $7 \mathrm{~min}$ (in case of $(-) \mu \mathrm{CP}$ ), or $10 \mathrm{~min}$ (in case of $(+) \mu \mathrm{CP}$ ). [CAUTION: potassium ferricyanide is light sensitive. Photodecomposition releases products that can contain free cyanide. Potassium ferricyanide is also incompatible with acids and liberates $\mathrm{HCN}]$.

Reactive ion etching: Reactive ion etching (RIE) experiments were carried out using an Electrotech Plasmafab 310-340 twin deposition/etch system to transfer the gold patterns into the underlying silicon. The RIE part consists of a parallel-plate system in an aluminum reactor, containing an $\mathrm{RF}$ generator operating at $13.56 \mathrm{MHz}$ and an automatic RF matching network. The pumping system consists of a turbopump in series with a rotary pump. The spacing between the electrodes is $8 \mathrm{~cm}$, with an RF electrode (styros) of $19 \mathrm{~cm}$ in diameter. The temperature of this target electrode was set at $10{ }^{\circ} \mathrm{C}$ and is controllable by backside heating/cooling using an oil bath system. Pressure control is possible in automatic mode (by the capacitive manometer) and in manual mode by adjusting the throttle valve position. Anisotropic etching of silicon was found for the following conditions: Automatic Pressure Control (APC) open, $50 \mathrm{~W}, 40 \mathrm{sccm} \mathrm{SF}_{6}$, and $20 \mathrm{sccm} \mathrm{O}_{2}$.

Characterization: PMMA film thickness determination was carried out with a Plasmos SD 2002 ellipsometer at a wavelength of $632.8 \mathrm{~nm}$. AFM imaging was carried out with a NanoScope III Multimode AFM (Digital Instruments, Santa Barbara, CA, USA) operating in contact mode using $\mathrm{Si}_{3} \mathrm{~N}_{4}$ cantilevers (Nanoprobes, 
Digital Instruments) with an approximate spring constant of $0.32 \mathrm{~N} \cdot \mathrm{m}^{-1}$. For height measurements, the scanner was calibrated in $\mathrm{z}$-direction using a calibration standard with known step height. Low-resolution SEM imaging was carried out with a JEOL JSM-5610 SEM operating in secondary electron detection. High-resolution SEM imaging was carried out with a LEO Gemini 1550 FEG-SEM.

\subsection{References and notes}

[1] Handbook of Microlithography, Micromachining and Microfabrication, Vol. 1 (Ed: P. RaiChoudhury), SPIE Optical Engineering Press, Bellingham, WA 1997.

[2] S. J. Holmes, P. H. Michel, M. C. Hakey, IBMJ. Res. Dev. 1997, 41, 7.

[3] R. H. Stulen, D. W. Sweeney, IEEE J. Quantum Elect. 1999, 35, 694.

[4] J. P. Silverman, J. Vac. Sci. Technol. B 1997, 15, 2117.

[5] M. A. McCord, J. Vac. Sci. Technol. B 1997, 15, 2125.

[6] U. S. Tandon, Vacuum 1992, 43, 241.

[7] (a) Y. Xia, G. M. Whitesides, Angew. Chem. Int. Ed. 1998, 37, 550.

(b) Y. Xia,G. M. Whitesides, Annu. Rev. Mater. Sci. 1998, 28, 153.

[8] S. Y. Chou, P. R. Krauss, P. J. Renstrom, Science 1996, 272, 85.

[9] K. Y. Suh, Y. S. Kim, H. H. Lee, Adv. Mater. 2001, 13, 1386.

[10] Y. Xia, G. M. Whitesides, Langmuir 1997, 13, 2059.

[11] Y. Xia, J. Tien, D. Qin, G. M. Whitesides, Langmuir 1996, 12, 4033.

[12] Y. Xia, G. M. Whitesides, Adv. Mater. 1995, 7, 471.

[13] Y. Xia, E. Kim, X.-M. Zhao, J. A. Rogers, M. Prentiss, G. M. Whitesides, Science 1996, 273, 347.

[14] Lithographic molding combines the patterning of gold by microcontact printing (printing and selective wet-chemical etching of the bare gold) with anisotropic wet etching of silicon in an alcoholic solution of $\mathrm{KOH}$. The resulting structures in silicon function as high-resolution masters for stamp fabrication. J. L. Wilbur, E. Kim, Y. Xia, G. M. Whitesides, Adv. Mater. $1995,7,649$.

[15] K. Y. Suh, H. H. Lee, Adv. Funct. Mater. 2002, 12, 405.

[16] For information on the effect of curing conditions on the material properties of PDMS stamps, see http:/www.zurich.ibm.com/st/microcontact/stamps/material.html (accessed at 25-11-2003).

[17] It was found in preliminary CFL experiments that either application of a low pressure $\left(1.6 \mathrm{~N} \cdot \mathrm{cm}^{-2}\right)$ on regular PDMS stamps or gradually increasing the temperature to the final 
annealing temperature (in combination with the use of low-modulus and flexible PDMS stamps) were successful measures to prevent separation, but at the expense of asymmetric CFL structures.

[18] K. Y. Suh, J. Park, H. H. Lee, J. Chem. Phys. 2002, 116, 7714.

[19] K. Y. Suh, P. Kim, H. H. Lee, Appl. Phys. Lett. 2004, 85, 4019.

[20] Y. Xia, X.-M. Zhao, E. Kim, G. M. Whitesides, Chem. Mater. 1995, 7, 2332.

[21] (a) J. A. Rogers, K. E. Paul, R. J. Jackman, G. M. Whitesides, Appl. Phys. Lett. 1997, 70, 2658. (b) T. W. Odom, V. R. Thalladi, J. C. Love, G. M. Whitesides, J. Am. Chem. Soc. 2002, $124,12112$.

[22] J. C. Love, K. E. Paul, G. M. Whitesides, Adv. Mater. 2001, 13, 604.

[23] J. Aizenberg, A. J. Black, G. M. Whitesides, Nature 1998, 394, 868.

[24] K.E. Paul, T.L. Breen, J. Aizenberg, G.M. Whitesides, Appl. Phys. Lett. 1998, 73, 2893.

[25] (a) E. Delamarche, H. Schmid, A. Bietsch, N. B. Larsen, H. Rothuizen, B. Michel, H. Biebuyck, J. Phys. Chem. B 1998, 102, 3324. (b) L. Libioulle, A. Bietsch, H. Schmid, B. Michel, E. Delamarche, Langmuir 1999, 15, 300.

[26] (a) A. Bietsch, B. Michel, J. Appl. Phys. 2000, 88, 4310. (b) H. Schmid, B. Michel, Macromolecules 2000, 33, 3042.

[27] E. Delamarche, M. Geissler, H. Wolf, B. Michel, J. Am. Chem. Soc. 2002, 124, 3834.

[28] H. Jansen, M. J. de Boer, R. J. Legtenberg, M. Elwenspoek, J. Micromech. Microeng. 1995, 5, 115.

[29] The definition of the (degree of) anisotropy is: anisotropy $=1-\frac{\left(d_{2}-d_{1}\right)}{2 \cdot h}$ with $d_{1}$ and $d_{2}$ the lateral width of the silicon feature at the base and top respectively, and $b$ the height of the silicon feature. 


\section{Fabrication of functional polymer templates as versatile tools for nanolithography*}

This chapter describes the fabrication of high-resolution polymer templates by capillary force lithography (CFL) and its implementation in nanoimprint lithography (NIL) and in the wetchemical etching of metals. Fabrication of gold patterns on silicon substrates with a lateral resolution of 100-150 nm demonstrates the potential of CFL templates as versatile tools for nanolithography.

\subsection{Introduction}

The development of low-cost, high-throughput lithographic techniques for the fabrication of sub-100 $\mathrm{nm}$ patterns has taken a dominant position in nanofabrication during the last decade. The unrelenting efforts that are undertaken in this field are the

* Part of this chapter has been published in: C. M. Bruinink, M. Péter, P. A. Maury, M. J. de Boer, L. Kuipers, J. Huskens, D. N. Reinhoudt, Adv. Funct. Mater. 2006, 16, 1555 (Copyright (C) 2006 Wiley-VCH Verlag $\mathrm{GmbH} \&$ Co. KGaA). 
result of the corresponding high cost and low accessibility of state-of-the-art lithographic techniques, e.g., deep-UV (DUV) and extreme-UV (EUV) photolithography, ${ }^{[1]}$ soft $\mathrm{X}$-ray lithography, ${ }^{[2]}$ electron-beam writing, ${ }^{[3]}$ and ion-beam lithography ${ }^{[4]}$ for large-area fabrication of high-resolution patterns. Although these techniques will remain the main technology for the semiconductor industry, soft lithography ${ }^{[5]}$ and nanoimprint lithography $(\mathrm{NIL})^{[6]}$ have made enormous progress as non-photolithographic techniques in several areas of exploratory and scientific research in terms of sub-100 nm resolution, reproducibility, and uniformity of the patterns over large areas. Generally, the concept of these non-photolithographic techniques lies in the exact replication of the structures on a master by (conformal) contact with the substrate of interest. To overcome the reliance on expensive state-of-the-art equipment for the fabrication of the necessary high-resolution templates, several innovative strategies have been put forward that extend the capabilities of soft lithography and NIL into the sub-100 nm range. These inexpensive strategies basically exploit the combination of different (aspects of) soft- and/or photo-lithographic techniques in order to downscale the feature sizes of the master. From the ever increasing list of existing strategies, the most promising set of lithographic techniques to date is edge lithography. The general feature that relates the lithographic techniques in this set is the translation of the structural characteristics of the master into resultant edge patterns. Edge-lithographic techniques include near-field phase-shifting photolithography, ${ }^{[7]}$ maskless lithography, ${ }^{[8]}$ undercutting by isotropic wet-chemical etching, ${ }^{[9]}$ patterning local disorder in monolayer resists, ${ }^{[10]}$ edge-spreading lithography, ${ }^{[11]}$ and multiplication by sidewall-patterning. ${ }^{[12,13]}$ The development of these techniques has led to the facile fabrication of features with ultimate lateral sizes down to $50 \mathrm{~nm}$.

A novel variant of edge lithography was shown in Chapter 4 by combining capillary force lithography $(\mathrm{CFL})^{[14,15]}$ of thin (with respect to the intrusion depth of the stamp) polymer layers with replica molding $(\mathrm{REM})^{[5]}$ for the fabrication of second-generation stamps suitable for conducting high-resolution soft lithography. ${ }^{[16]}$ Although no special attention was given to the resulting high-resolution structures by Lee et al., ${ }^{[14]}$ it was shown in Chapter 4 that these polymer templates have potential in edge lithography, for example, the use of these templates as masters in soft lithography 
(Scheme 5.1, route A). Fabrication of gold patterns with a resolution of $\sim 125 \mathrm{~nm}$ was feasible by application of the resulting stamps in microcontact printing $(\mu \mathrm{CP})$ and subsequent wet-chemical etching. ${ }^{[16]}$

This chapter reports recent developments of CFL as a nanofabrication technique by utilizing the resulting CFL polymer templates in two additional patterning techniques (Scheme 5.1): as molds for NIL (Scheme 5.1, route B) and as etch masks for the selective wet-chemical etching of metals (Scheme 5.1, route C) by incorporation of a $\mathrm{UV}$-curable polymer and the use of gold-on-silicon substrates in the CFL procedure, respectively.

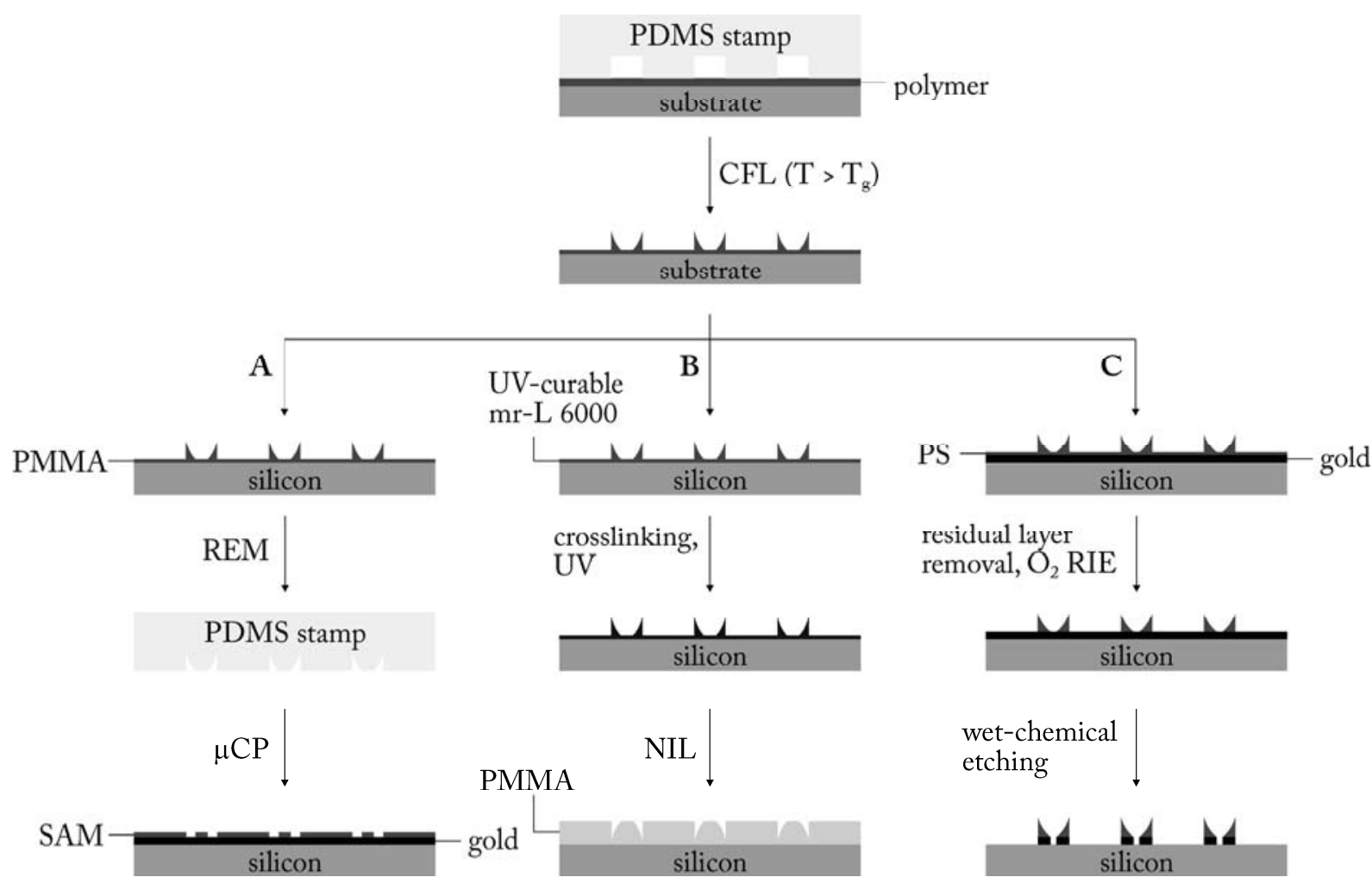

Scheme 5.1 Schematic representation of the different functions of a high-resolution CFL template depending on the type of polymer and substrate: (A) template as master for stamp fabrication; (B) template as mold for NIL; (C) template as etch mask for the wet-chemical etching of metals.

The (mechanical) stability and integrity of the template during processing and the ultimate resolution of the resulting metal patterns are of prime importance in our process evaluation, which was carried out by atomic force microscopy (AFM) and scanning electron microscopy (SEM). Therefore, emphasis was put on the process 
conditions and the etch behavior of the polymer templates (and of the underlying substrate) in order to achieve these goals.

\subsection{Results and discussion}

\subsubsection{CFL templates as high-resolution molds for NIL}

The requirements for polymer templates to successfully function as robust NIL molds (Scheme 5.1, route B) are more stringent than for use as masters for $\mu \mathrm{CP}$ stamp fabrication (Scheme 5.1, route A), because the imprint conditions in standard NIL processing schemes demand the template to be resistant to high temperatures - in the range of $70-80{ }^{\circ} \mathrm{C}$ above the $\mathrm{T}_{\mathrm{g}}$ of the polymer - and high pressures for several minutes.

To date, only low-viscosity thermoplastic polymers, e.g., poly(methylmethacrylate) (PMMA), polystyrene (PS), and some block copolymers have been exploited in CFL. However, the CFL process is universal in that it also allows patterning of other types of polymers, e.g., UV-curable polymers. The use of this type of polymers offers the advantage over thermoplastic polymers of post-processing stabilization of the polymer template by UV crosslinking (Fig. 5.1a).

(a)

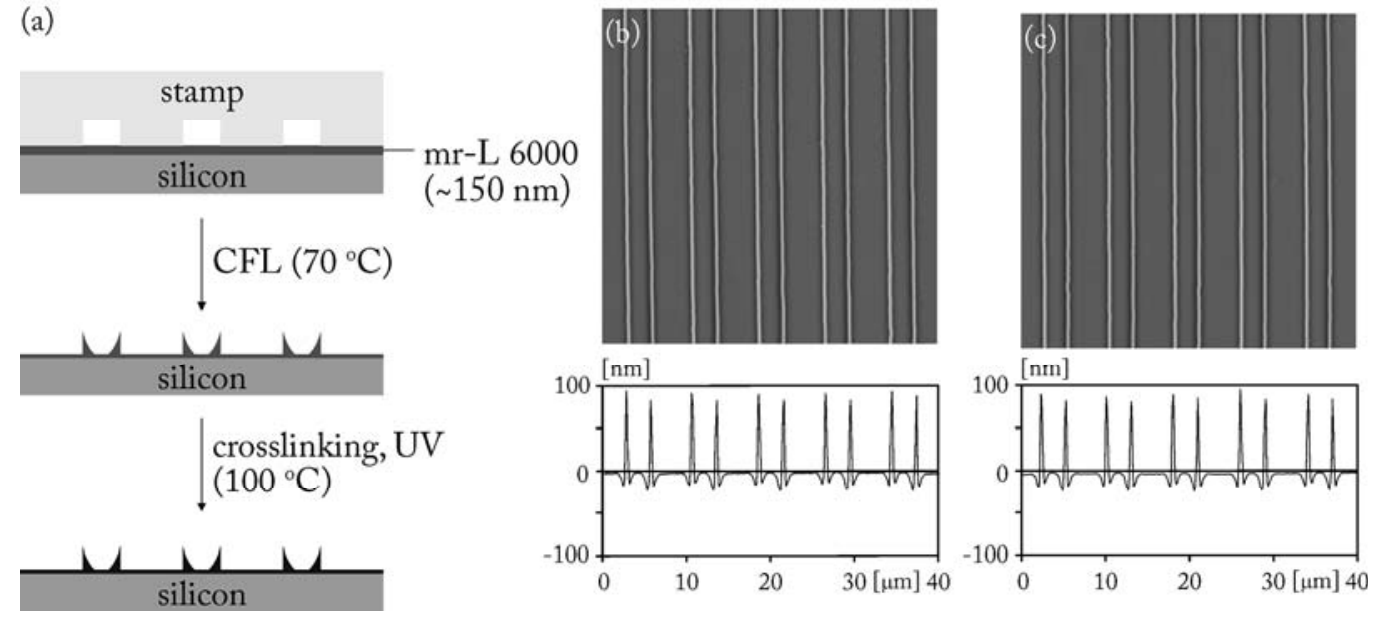

Figure 5.1 (a) Schematic representation of the CFL procedure for the fabrication of NIL molds using the UVcurable resist mr-L 6000. (b/c) CM AFM height images (z range: $800 \mathrm{~nm}$; image size: $40 \mu \mathrm{m} \times 40 \mu \mathrm{m}$ ) of the high-resolution polymer template before (left) and after UV crosslinking (right), including the average height cross-sectional analyses. The polymer template was fabricated by CFL on a $150 \mathrm{~nm} \mathrm{mr}-\mathrm{L} 6000$ layer at $70{ }^{\circ} \mathrm{C}$ for $2 \mathrm{~h}$. Subsequently, the template was exposed to a dose of $360 \mathrm{~mJ} \mathrm{~cm}^{-2}$ (at an intensity of $9 \mathrm{~mW}^{\cdot} \mathrm{cm}^{-2}$ ) in order to induce crosslinking on a hotplate at $100{ }^{\circ} \mathrm{C}$ for $10 \mathrm{~min}$. 


\subsubsection{UV crosslinking for post-processing stabilization}

The commercially available UV-curable polymer mr-L 6000 is a chemically amplified negative-tone photoresist with a very low glass-transition temperature $\left(\mathrm{T}_{\mathrm{g}}\right)$ of $35^{\circ} \mathrm{C}$, permitting moderate processing conditions for patterning by $\mathrm{CFL}$ $\left(\mathrm{T}=70^{\circ} \mathrm{C}, 2 \mathrm{~h}\right.$ ). Special care was taken that, prior to UV exposure, the mr-L 6000 was not exposed to white light (i.e., processing was only carried out in a yellow light environment). Cross-sectional AFM analysis of a representative mr-L 6000 template (Fig. 5.1b) reveals the typical transient meniscus (W-shape) of capillary rise. ${ }^{[17,18]}$ It has been shown that (i) any meniscus evolves in a uniform U-shape upon long annealing times, ${ }^{[17]}$ and that (ii) splitting of the meniscus may give rise to exposure of the underlying substrate in the noncontact areas. ${ }^{[18]}$ Therefore, the observation of a transient meniscus in our template implies that, under the present processing conditions, the polymer pattern could not fully develop the characteristic U-shape meniscus.

UV crosslinking of mr-L 6000 templates typically involves a flood-exposure in a UV exposure system, followed by a post-exposure bake on the hotplate. For an exposure dose of $360 \mathrm{~mJ} \cdot \mathrm{cm}^{-2}$ and a post-exposure bake at $100{ }^{\circ} \mathrm{C}$ for $10 \mathrm{~min}$, the structural integrity of the template was not affected by this post-processing stabilization procedure (Fig. $5.1 \mathrm{~b} / \mathrm{c}$, template height is $\sim 100 \mathrm{~nm}$ and FWHM is $\sim 400 \mathrm{~nm}$ ). UV exposure of this type of resist activates a photo-acid generator responsible for releasing an acid that initiates the cationic polymerization of the epoxy resist upon heating. At this stage, both crosslinking and thermal flow may occur simultaneously, which can have a significant effect on the pattern fidelity upon curing, in particular in case thermal flow dominates over crosslinking. ${ }^{[19]}$ The results shown in Fig. 5.2 are the outcome of an AFM study that was carried out in order to address the interplay between these two processes during a post-exposure bake at $100{ }^{\circ} \mathrm{C}$ for $10 \mathrm{~min}$ in relation to the exposure dose.

The response of unexposed mr-L 6000 templates to thermal treatment excludes the interference of thermal crosslinking with the stabilization of polymer patterns during UV-crosslinking at $100{ }^{\circ} \mathrm{C}$ (Fig. 5.2a). Fig. 5.2 illustrates a clear trend in pattern stability, in terms of feature height and width, as a function of the exposure dose. 

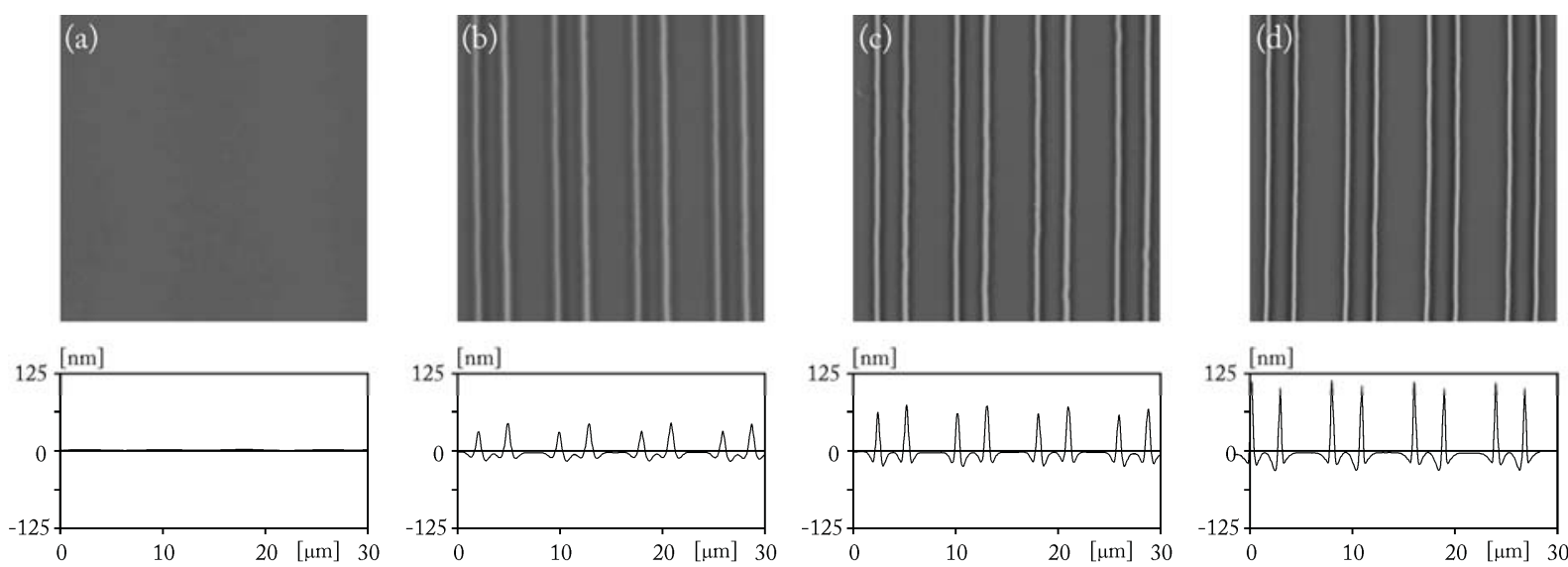

(e)

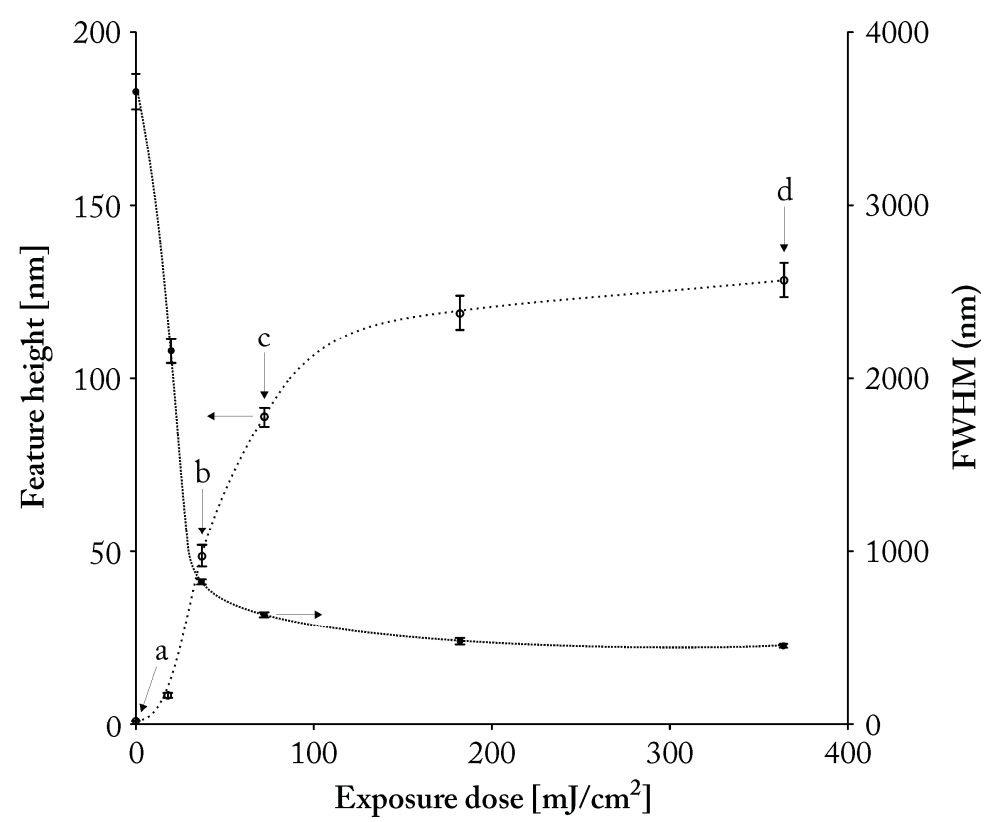

Figure 5.2 Exposure dose dependence of the stability of the polymer templates (CFL at $70{ }^{\circ} \mathrm{C}$ for $2 \mathrm{~h}$ ) towards a standard post-exposure bake at $100^{\circ} \mathrm{C}$ for $10 \mathrm{~min}$ as shown by CM AFM height images (z range: $250 \mathrm{~nm}$; image size: $30 \mu \mathrm{m} \times 30 \mu \mathrm{m}$ ), including the average height cross-sectional analyses, after no exposure (a), and at exposure doses of $36 \mathrm{~mJ} \cdot \mathrm{cm}^{-2}$ (b), $72 \mathrm{~mJ} \cdot \mathrm{cm}^{-2}$ (c), and $360 \mathrm{~mJ} \cdot \mathrm{cm}^{-2}$ (d). (e) Plot of the feature height and FWHM of the polymer template as a function of the exposure dose. The trend lines in the plot are only drawn as a guide to the eye; a-d refer to the corresponding AFM images.

At low exposure doses thermal flow was the dominating process, causing significant sagging of the features of the template (Fig. 5.2b). Upon increasing the exposure dose (Fig. 5.2c) the template structures became more and more heat resistant up to the point that the feature dimensions were independent of the exposure dose (Fig. 5.2d). At these high exposure doses, the polymer template was resistant to thermal flow as crosslinking was the dominating process in this regime. The critical exposure dose of 
$\sim 100 \mathrm{~mJ} \cdot \mathrm{cm}^{-2}$, at which UV crosslinking of the polymer template is still successful, was estimated from the plot in Fig. 5.2e. Quantitatively, this exposure dose $\left(100 \mathrm{~mJ} \cdot \mathrm{cm}^{-2}\right.$ for $150 \mathrm{~nm}$ thick layers) is $33 \%$ larger in comparison to the one from dissolution experiments of full resist layers after exposure $\left(500 \mathrm{~mJ} \cdot \mathrm{cm}^{-2}\right.$ for $1 \mu \mathrm{m}$-thick layers). ${ }^{[19]}$ However, no difference in pattern stability between templates that have undergone a standard post-exposure bake $\left(100{ }^{\circ} \mathrm{C}\right.$ for $\left.10 \mathrm{~min}\right)$ and a gradual post-exposure bake ${ }^{[19]}$ (from $30{ }^{\circ} \mathrm{C}$ to $100{ }^{\circ} \mathrm{C}$ with a ramp of $\sim 4{ }^{\circ} \mathrm{C} \cdot \mathrm{min}^{-1}$ ) was found by AFM analysis. This result implies that the initiation of crosslinking on the hot plate is rather fast on the timescale of thermal flow, and therefore that the rate of crosslinking (resulting in pattern stability) is totally dependent on the exposure dose.

\subsubsection{Thermal imprinting and pattern transfer}

An additional long exposure and hard bake at $120{ }^{\circ} \mathrm{C}$ for $10 \mathrm{~min}$ was carried out prior to imprinting in PMMA to further increase the thermal and mechanical stability. The structural integrity of the mold was not affected by this hard bake. At a FWHM of $\sim 400 \mathrm{~nm}$, the pattern density of the as-fabricated polymer mold was about $10 \%$. For imprinting of such low-pattern-density molds in high-molecular weight PMMA $(\mathrm{Mw}=350 \mathrm{kDa})$, the NIL conditions were set to $180{ }^{\circ} \mathrm{C}$ and $100 \mathrm{bar}$ at an imprint time of $1 \mathrm{~min}$ (Fig. 5.3a). Figs. 5.3b/c illustrate the high fidelity of NIL as a technique for the pattern replication of the polymer mold in (thermoplastic) PMMA. The feature dimensions of this polymer imprint (Fig. 5.3c, $120 \mathrm{~nm}$ in height and FWHM of $390 \mathrm{~nm}$ ) approximate the dimensions of the polymer mold (Fig. 5.3b, $125 \mathrm{~nm}$ in height and a FWHM of $400 \mathrm{~nm}$, fabricated by CFL at $70{ }^{\circ} \mathrm{C}$ for $4 \mathrm{~h}$ ).

AFM analysis of the mold during a durability test of tens of imprints (Figs. 5.3b and 5.3d) also illustrates the high mechanical stability of the mold to imprinting under the present conditions. The anti-adhesive properties of this low-density polymer mold were also outstanding. Specific efforts that were undertaken in the past to eliminate the adhesion of polymer to the mold include the use of ultrathin anti-adhesive layers by plasma deposition, ${ }^{[20]}$ anti-adhesion additives in polymers, ${ }^{[21]}$ and the formulation of novel resists and low-surface energy mold materials. An additive, the formulation of mr-L 6000, or a combination of both factors could account for the excellent antiadhesive properties of the polymer molds during NIL in our case. ${ }^{[22]}$ 
(a)
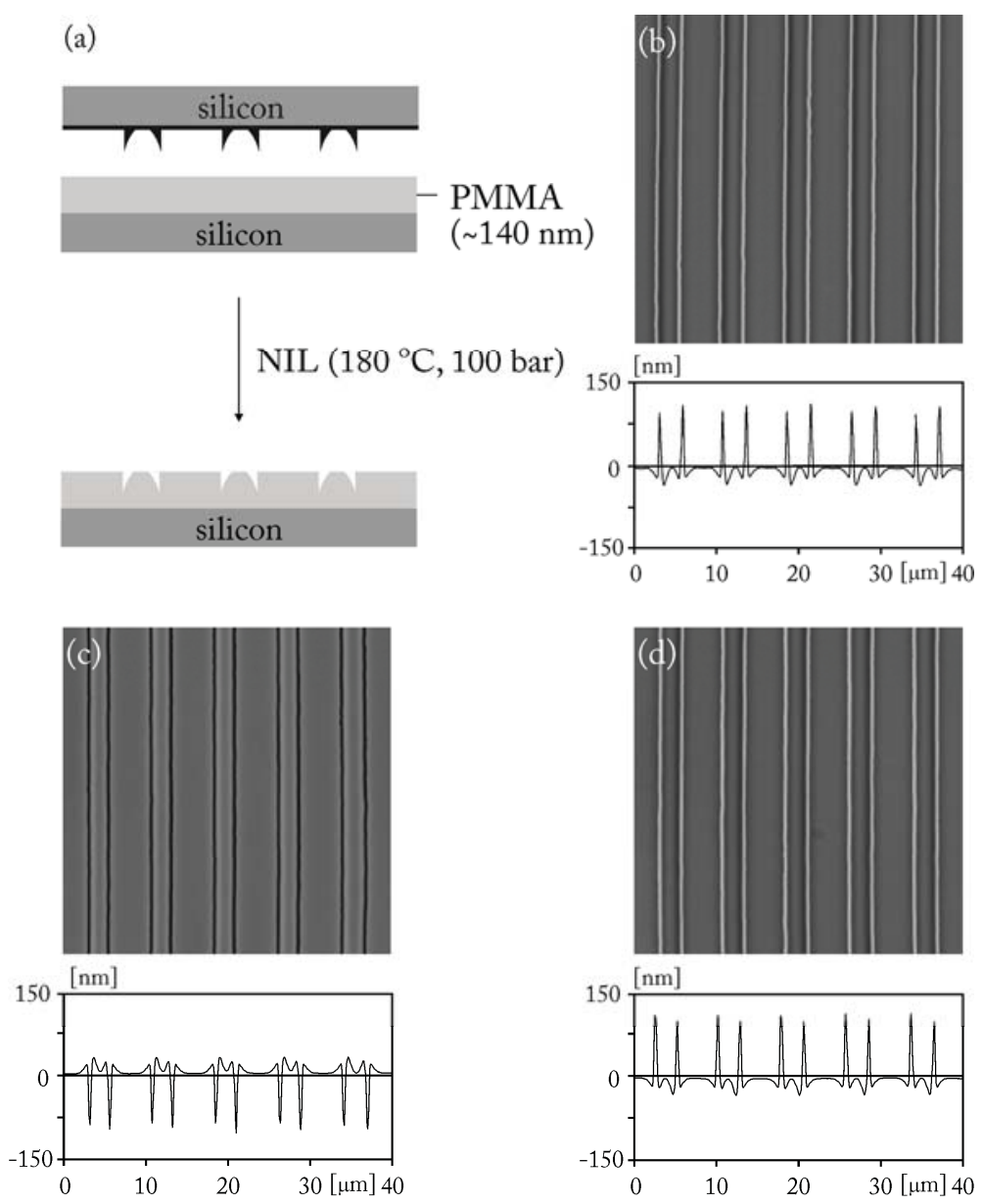

Figure 5.3 (a) Schematic representation of the NIL procedure with CFL molds. (b/c/d) CM AFM height images ( $\mathrm{z}$ range: $300 \mathrm{~nm}$; image size: $40 \mu \mathrm{m} \times 40 \mu \mathrm{m}$ ) of the polymer mold (b), an imprint in PMMA (c), and the same polymer mold after tens of imprints at $180^{\circ} \mathrm{C}$ and $100 \mathrm{bar}(\mathrm{d})$, including average height cross-sectional analyses.

In most NIL applications, additive (deposition) or subtractive (etching) postimprint processing steps usually follow the imprinting step. To use the functionality of the imprint as a template, it is essential to remove the residual polymer layer in the trenches of the imprint by $\mathrm{O}_{2}$ reactive ion etching $\left(\mathrm{O}_{2} \mathrm{RIE}\right)$. The thickness of this residual layer was kept to a minimum by choosing the thickness of the initial PMMA layers $(\sim 140 \mathrm{~nm})$ close to the imprint depth of the polymer mold. Cross-sectional AFM analysis of the imprint (Fig. 5.4a) illustrates a positive taper that is, in general, not the ideal lift-off profile ${ }^{[23]}$ for performing metal lift-off. To prevent tearing and detachment of the metal during lift-off, metal lift-off on these imprints was performed for thin metal layers only ( $10 \%$ of the imprint depth). Special care was taken to position the substrate vertically to the evaporation source during the evaporation of 
$10 \mathrm{~nm}$ of gold. Subsequent sonication in warm acetone was carried out to remove the polymer template. Gold lines of $\sim 150 \mathrm{~nm}$ wide were fabricated on silicon using this procedure (Fig. 5.4b). The imprint can also function as an etch mask in case the imprinting is done on continuous metal layers on silicon substrates. $150 \mathrm{~nm}$-wide trenches were fabricated on gold-on-silicon substrates by wet-chemical etching the gold using a basic ferro/ferricyanide-containing etch solution ${ }^{[24]}$ after the $\mathrm{O}_{2} \mathrm{RIE}$ (Fig. 5.4c).
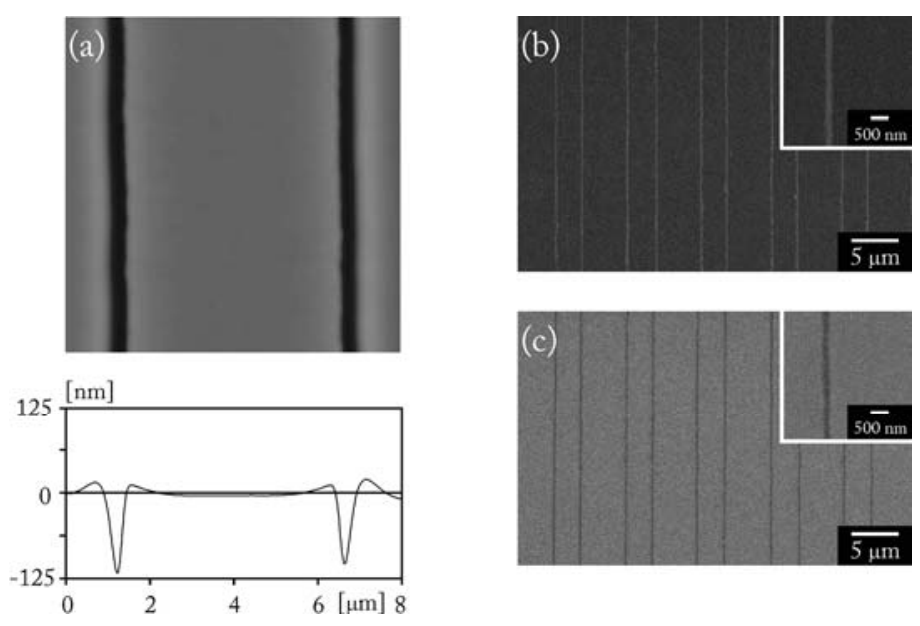

Figure 5.4 (a) CM AFM height image (z range: $250 \mathrm{~nm}$; image size: $8 \mu \mathrm{m} \times 8 \mu \mathrm{m}$ ) of a PMMA imprint, including the average height cross-sectional analysis. The imprint was fabricated by imprinting with a CFL mold in $140 \mathrm{~nm}$ PMMA at $180{ }^{\circ} \mathrm{C}$ and 100 bar. The processing time for $\mathrm{O}_{2} \mathrm{RIE}\left(50 \mathrm{mTorr}, 50 \mathrm{~W}, 20 \mathrm{sccm} \mathrm{O}_{2}\right.$, $15-20 \mathrm{~s}$ ) was set to over-etch $\sim 20 \%$ of the actual residual layer thickness in order to correct for any differences in residual layer thickness over the entire area of the imprint (etch rate of $350 \mathrm{kDa}$ PMMA is $\sim 1.7 \mathrm{~nm}^{\bullet} \mathrm{s}^{-1}$ ). (b/c) SEM images of $150 \mathrm{~nm}$-wide gold lines on silicon after subsequent evaporation of $10 \mathrm{~nm}$ of gold and sonication of the substrate in acetone at $40{ }^{\circ} \mathrm{C}$ for $15 \mathrm{~min}(\mathrm{~b})$ and of $150 \mathrm{~nm}$-wide trenches in gold-on-silicon substrates after wet-chemical etching for $10 \mathrm{~min}$ and removal of the PMMA in acetone (c). The inserts show the higher magnification SEM images of the individual features.

As a result of the positive taper in the PMMA imprint, a discrepancy exists between the feature dimensions of this imprint (after $\mathrm{O}_{2} \mathrm{RIE}, \mathrm{FWHM}$ of $\sim 400 \mathrm{~nm}$ ) and the feature dimensions of the metal structures. By changing the time of $\mathrm{O}_{2} \mathrm{RIE}$ treatment, it should, in principal, be feasible to create different opening sizes to the substrate, resulting in further reduction of the feature dimensions of the metal structures after post-processing (e.g., metal lift off, or wet-chemical etching) to the sub-100 nm scale. Therefore, these results do not represent the lower limit of this approach, as NIL has 
been proven to be the most promising technology for the replication of sub-10 nm features. ${ }^{[25]}$

\subsubsection{CFL templates as etch masks in wet-chemical etching of metals}

One of the major drawbacks of most polymers is the poor etch resistivity toward $\mathrm{O}_{2}$ (containing) plasmas. Recently, poly(ferrocenylsilanes) (PFS) ${ }^{[26]}$ with high etch resistance have been exploited in the CFL processing for the direct transfer of the template pattern into silicon by reactive ion etching. ${ }^{[27]}$ The requirements for polymer templates to function as etch masks in the wet-chemical etching of metals are, however, not so stringent. This procedure (Scheme 5.1, route C) differs from the fabrication of $\mu \mathrm{CP}$ masters (Scheme 5.1, route $\mathrm{A}$ ) in that $\mathrm{CFL}$ of thermoplastic polymers, in this case PMMA $(\mathrm{Mw}=38 \mathrm{kDa})$ and PS $(\mathrm{Mw}=240 \mathrm{kDa})$, is carried out on $20 \mathrm{~nm}$ gold-on-silicon substrates instead of on bare silicon substrates (Fig. 5.5a).
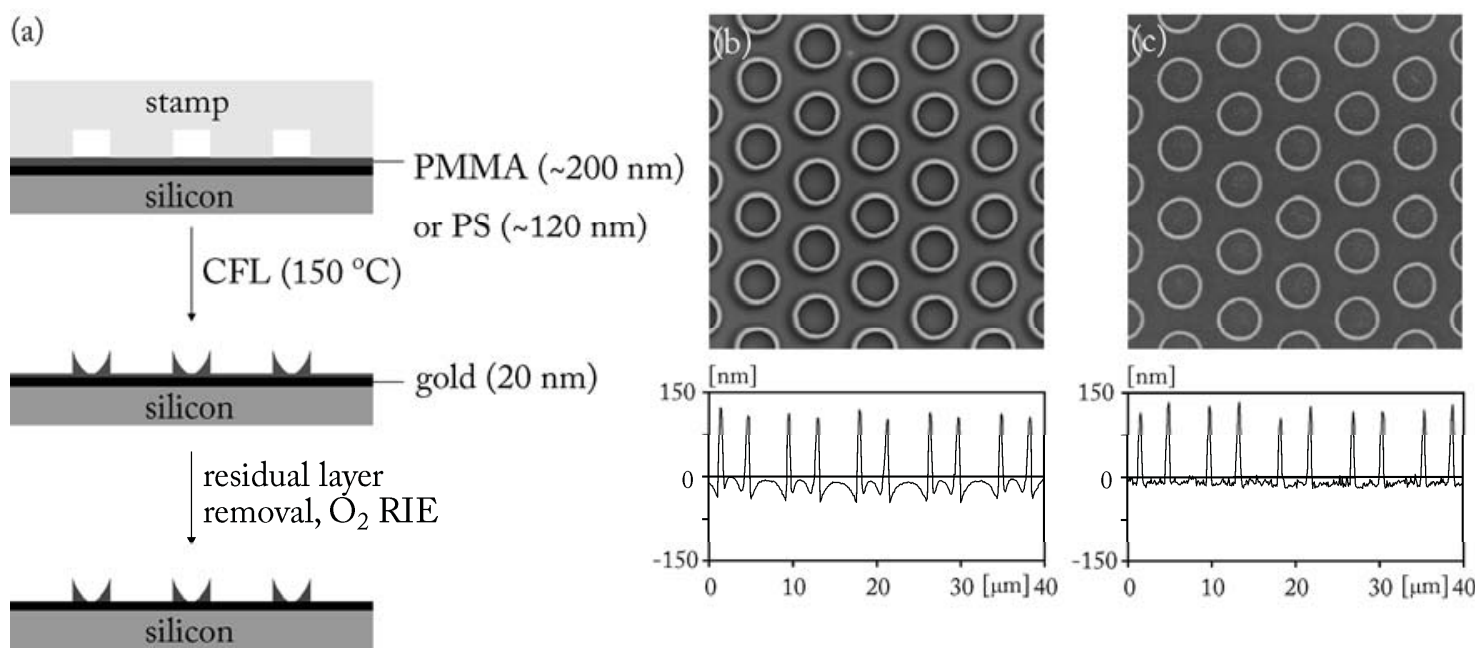

Figure 5.5 (a) Schematic representation of the CFL procedure for the fabrication of polymer (PMMA or PS) etch masks on gold-on-silicon substrates. (b/c) CM AFM height images (z range: $300 \mathrm{~nm}$; image size: $40 \mu \mathrm{m} \times 40 \mu \mathrm{m}$ ) of a PS template (b) and the corresponding etch mask after residual layer removal (c), including the cross-sectional analyses. The PS template was fabricated by CFL on a $\sim 120 \mathrm{~nm}$ PS layer at $150{ }^{\circ} \mathrm{C}$ for $8 \mathrm{~h}$. Removal of the residual layer was done by $\mathrm{O}_{2} \mathrm{RIE}$ for $2 \mathrm{~min}$ in order to expose the underlying gold. This processing time was set to over-etch $\sim 20 \%$ of the actual residual layer thickness in order to correct for any differences in residual layer thickness over the entire area of the template.

Cross-sectional AFM analysis of a representative PS template (Fig. 5.5b) illustrates again the typical transient meniscus (W-shape) of capillary rise. The present processing conditions result in polymer template features of $180 \mathrm{~nm}$ high (FWHM is 
$\sim 480 \mathrm{~nm}$ ). Attempts to etch the underlying gold were futile, due to the presence of a residual layer that was protecting the gold. For this template (Fig. 5.5b), the residual layers was on the order of $80 \mathrm{~nm}$, as determined by an AFM height measurement of the polymer template at a scratch made with a scalpel. Removal of the residual layer without deterioration of the template structures is crucial in the present procedure. The gradual dissolution of the residual polymer in acetone was found to comprise a convenient and fast procedure as polymer templates dissolve within about 10-15 s. However, $\mathrm{O}_{2} \mathrm{RIE}$ was chosen instead in order to have more control over the removal rate of the polymer, which is of the order of $n \mathrm{~nm}^{-1}$. The $\mathrm{O}_{2} \mathrm{RIE}$ etch rates of PS and PMMA were $0.9 \mathrm{~nm} \cdot \mathrm{s}^{-1}$ and $1.9 \mathrm{~nm} \cdot \mathrm{s}^{-1}$, respectively, as determined from film thickness measurements by ellipsometry on full polymer layers upon etching.

\subsubsection{Effect of PDMS transfer on the template processability}

A distinct difference in adhesion of PDMS stamps to PS and PMMA was seen upon cooling of the substrate plus stamp, which appears to result in the clear $\mathrm{O}_{2}$ RIE rate difference found upon removal of the residual layer. PDMS stamps were found to detach from PS templates within seconds whereas manual removal of the stamps was necessary in the case of PMMA templates. For the PS templates, removal of the residual layer was feasible at a process time corresponding to the etch rate for full layers. From the cross-sectional AFM analysis (Fig. 5.5c), it is clear that the PS template structure was still intact (in terms of the profile) after $\mathrm{O}_{2}$ RIE. Additionally, the absolute reduction of $\sim 50 \mathrm{~nm}$ in feature height and FWHM to $\sim 130 \mathrm{~nm}$ and $\sim 430 \mathrm{~nm}$, respectively, indicates that the $\mathrm{O}_{2}$ RIE was isotropic. For the PMMA templates, the complete removal of the residual layer in the former contact areas of the stamp with the PMMA substrate was impossible (Fig. 5.6a/b), even after longer etch times. The contrasting etching behavior of these different polymer templates in $\mathrm{O}_{2}$ RIE was the starting point for investigating the templates and the polymer etch rates in more detail by AFM friction analysis, X-ray photoelectron spectroscopy (XPS), and ellipsometry. Only in the case of PMMA was a significant increase in friction in the contact areas found by AFM friction analysis (Fig. 5.6c). This was attributed to the transfer of low-molecular-weight silicone residues from the PDMS stamp onto the template during CFL of PMMA. Silicon-containing polymers are 
known to form thin, non-volatile silicon oxide $\left(\mathrm{SiO}_{2}\right)$ layers at the surface during $\mathrm{O}_{2}$ plasma exposure and therefore exhibit a high etch resistance toward $\mathrm{O}_{2} \mathrm{RIE}$. ${ }^{[28]}$
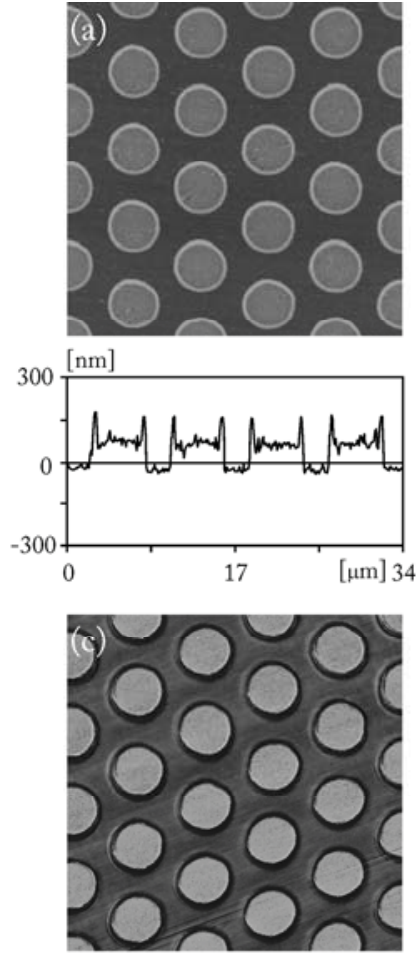

(b)

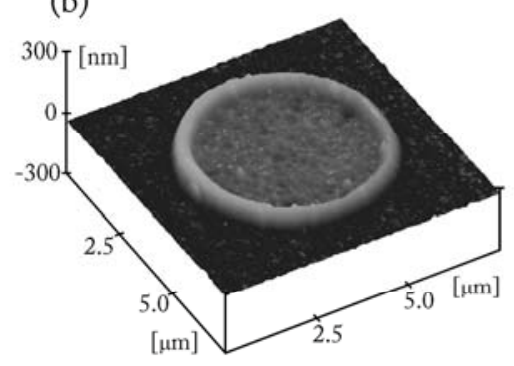

(d)

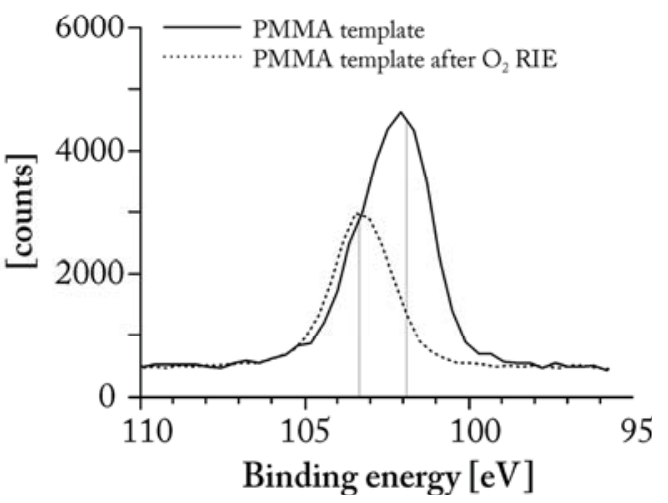

Figure 5.6 (a) CM AFM height image (z range: $600 \mathrm{~nm}$; image size: $34 \mu \mathrm{m} \times 34 \mu \mathrm{m}$ ) of a PMMA template (CFL at $150^{\circ} \mathrm{C}$ for $8 \mathrm{~h}$ ) after $100 \mathrm{~s}$ of $\mathrm{O}_{2} \mathrm{RIE}$, including the cross-sectional analysis. (b) Three-dimensional CM AFM height image (z-range: $600 \mathrm{~nm}$; image size: $7.5 \mu \mathrm{m} \times 7.5 \mu \mathrm{m}$ ) of a close-up of one individual PMMA ring. (c) CM AFM friction image of a PMMA template that was in contact with a minimal-modulus PDMS stamp for $8 \mathrm{~h}$ at $150{ }^{\circ} \mathrm{C}$, prior to $\mathrm{O}_{2} \mathrm{RIE}$ (image size: $34 \mu \mathrm{m} \times 34 \mu \mathrm{m}$; friction forces [a.u.] increase from dark to bright contrast). (d) $\mathrm{Si}(2 \mathrm{p})$ XPS spectra of full PMMA layers before and after $\mathrm{O}_{2}$ RIE. The full polymer layers in this typical experiment were in contact with featureless PDMS stamps under the same CFL conditions. The vertical lines in the spectra represent the positions of the $\mathrm{Si}(2 \mathrm{p})$ peak for PDMS $(101.8 \mathrm{eV})^{[29]}$ and $\mathrm{SiO}_{2}$ $(103.3 \mathrm{eV}){ }^{[33]}$

To examine the transfer of silicone residues and its effects on the polymer etch rate, $\sim 150 \mathrm{~nm}$ thick PS and PMMA layers were put in contact with two featureless PDMS stamps of different modulus at $150{ }^{\circ} \mathrm{C}$ for $8 \mathrm{~h}$ (standard CFL conditions). X-ray photoelectron spectroscopy (XPS) analysis was performed on these layers to determine the amount of silicone transfer, whereas ellipsometry was performed to determine the corresponding etch rate during $20 \mathrm{~s}$ of $\mathrm{O}_{2} \mathrm{RIE}$. The results are shown in Table 5.1. 
Table 5.1 XPS analysis of polymer substrates versus substrates that were in contact with featureless PDMS stamps under CFL process conditions $\left(150{ }^{\circ} \mathrm{C}, 8 \mathrm{~h}\right)$, and the corresponding etch rates for the polymers in $\mathrm{O}_{2}$ RIE (50 mTorr, $50 \mathrm{~W}, 20 \mathrm{sccm} \mathrm{O}_{2}, 20 \mathrm{~s}$ ).

\begin{tabular}{|c|c|c|c|c|c|}
\hline & & & XPS [\%] & & Etch rate \\
\hline & & $\mathrm{C}(1 \mathrm{~s})$ & $\mathrm{O}(1 \mathrm{~s})$ & $\operatorname{Si}(2 p)$ & {$[\mathrm{nm} \cdot \mathrm{s}]$} \\
\hline PMMA & no stamp & 71.4 & 28.6 & 0.0 & 1.9 \\
\hline & medium-modulus stamp & 67.1 & 29.1 & 3.8 & 1.6 \\
\hline & minimal-modulus stamp & 62.4 & 29.7 & 8.0 & 1.4 \\
\hline PS & no stamp & 99.5 & 0.5 & 0.0 & 0.87 \\
\hline & medium-modulus stamp & 96.8 & 1.8 & 1.4 & 0.81 \\
\hline & minimal-modulus stamp & 97.4 & 1.4 & 1.3 & 0.80 \\
\hline
\end{tabular}

For the PS $(\mathrm{C} / \mathrm{O}=1: 0.005)$ and PMMA $(\mathrm{C} / \mathrm{O}=5: 2.01)$ reference layers, the elemental atomic compositions are in close-to-theoretical amounts (PS $\mathrm{C} / \mathrm{O}=1: 0$, PMMA C/O = 5:2). On these reference polymer layers, no Si-containing residues were detected by XPS that could originate from the ambient (e.g., wafer dust, oil vapor from vacuum pumps). The presence and the position of the $\mathrm{Si}(2 \mathrm{p})$-peak $(102.1 \mathrm{eV})$ on each polymer layer that was in contact with a PDMS stamp indicates that contamination of the polymer surface with Si-containing species from the PDMS stamp $(101.8 \mathrm{eV})^{[29]}$ originates only from the CFL process, irrespective of the type of polymer and the type of PDMS stamp.

The most important findings of this XPS study are: (i) the transfer of Si-containing residues onto PMMA is three to six times higher than in the case of PS; (ii) the curing conditions of the PDMS stamp only affect the transfer in the case of PMMA (i.e., more curing results in less contamination); (iii) the presence of Si-containing residues on polymer layers compromises the processability of the polymer etch mask to a certain extent during the $\mathrm{O}_{2}$ RIE, resulting in etch rate reductions of $8 \%$ for PS and 16-26\% for PMMA. On the basis of the AFM height data in Fig. 5.6a, however, the reduction in etch rate on the PMMA template should have been at least $60 \% \cdot{ }^{[30]}$ This discrepancy suggests, in accordance with a previous study on other systems, ${ }^{[31]}$ that 
even more Si-containing residues are transferred in the case of stamps containing patterns. The fact that complete inhibition of etching is not seen in the case of PMMA indicates that the amount of Si-containing residues on the surface is not sufficient to form a continuous, etch-protective layer of $\mathrm{SiO}_{2 \cdot}{ }^{[32]}$ The clear shift of the $\mathrm{Si}(2 \mathrm{p})$-peak of the Si-containing residues to a higher binding energy $(103.4 \mathrm{eV})$, which is consistent with that of $\mathrm{SiO}_{2}{ }^{[33]}$ (Fig. 5.6d), and the high surface roughness ${ }^{[34]}$ $(\sim 20-40 \mathrm{~nm})$ in the former contact areas (Fig. 5.5b) upon $\mathrm{O}_{2}$ plasma exposure support this statement.

\subsubsection{Effect of over-etching on the feature dimensions}

On the basis of the processability by $\mathrm{O}_{2} \mathrm{RIE}$, PS was chosen as the polymer of choice in this procedure. Fig. 5.7b shows a SEM image of $390 \mathrm{~nm}$ wide gold rings on silicon after wet-chemical etching of the underlying gold for $10 \mathrm{~min}$. Over-etching and undercutting by isotropic wet-chemical etching are well-known techniques in the semiconductor industry to decrease feature sizes. ${ }^{[35]}$ Fig. 5.7 a shows our over-etching procedure for downscaling the lateral dimensions of the resulting metal structures under the polymer etch mask to the sub-100 nm range (Fig. 5.8c; average line width: $115 \mathrm{~nm}) \cdot{ }^{[36]}$

(a)
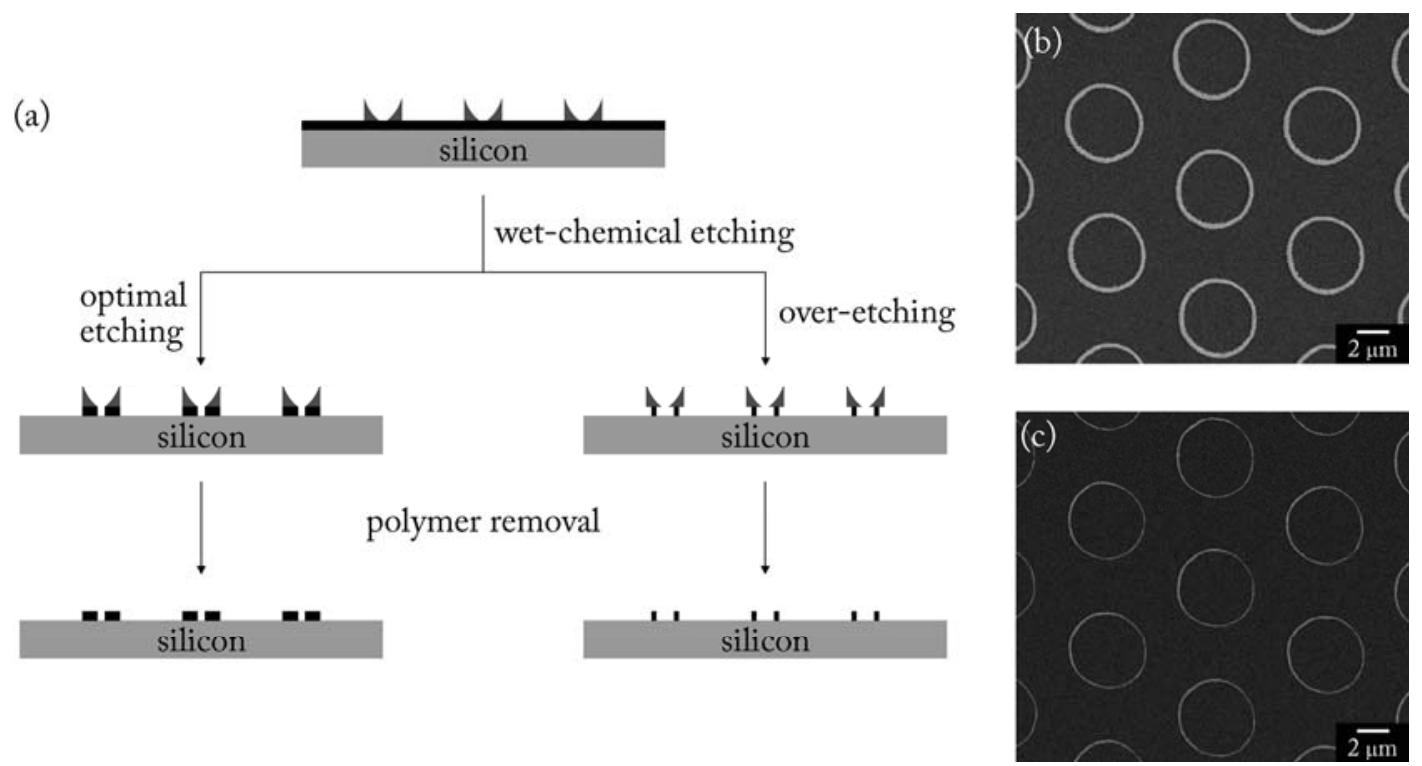

Figure 5.7 (a) Schematic representation of the wet chemical (over)etching procedure. Prior to wet-chemical etching, the gold was reduced in absolute ethanol for $30 \mathrm{~min}$. (b/c) SEM images of gold rings on silicon after wet-chemical etching of gold for $10 \mathrm{~min}$ and $60 \mathrm{~min}$, respectively, at room temperature, and subsequent PS removal by sonication in warm acetone for $10 \mathrm{~min}$. 
The basic concept of this procedure is to over-etch the gold from both sides of the sub-micrometer polymer etch mask to obtain high-resolution metal patterns. This procedure is therefore conceptually different from the procedure that exploits the undercut by isotropic wet-chemical etching to transfer the edges of polymer patterns by metal deposition and lift-off. ${ }^{[9]}$ The two SEM images in Fig. 5.7 illustrate the potential of the present over-etching procedure by the reduction of the lateral dimensions by a factor of 3.6. Additionally, the plot of the line width of the gold features versus the etch time (Fig. 5.8a) and the SEM images (Figs. 5.8b-e) illustrate that high control over the line width of the metal patterns is achieved by the etch time.

(a)

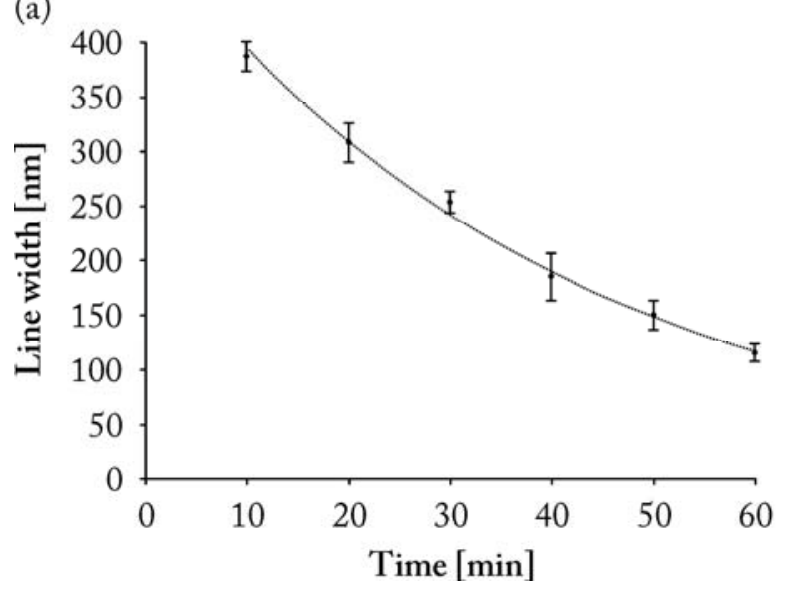

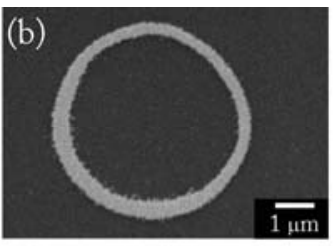

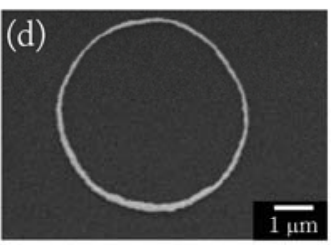

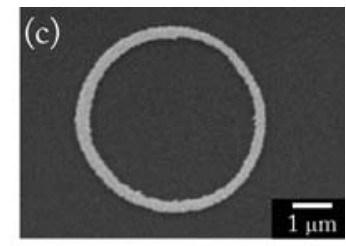

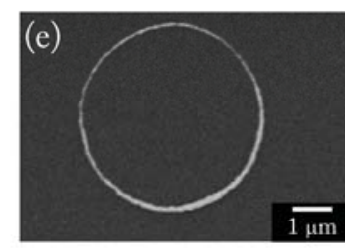

Figure 5.8 (a) Plot of the line width of the gold features versus the etch time at room temperature. The trend line in the plot is only drawn as a guide to the eye. Each data point was calculated from 6 rings (10 data points per ring) at different locations on the substrate (with a distance of $2.5 \mathrm{~mm}$ between each of these rings). The SEM images (b-e) demonstrate gold rings after wet-chemical etching of the gold for $10 \mathrm{~min}$ (b), $20 \mathrm{~min}$ (c), 50 $\min (\mathrm{d})$, and $60 \min (\mathrm{e})$.

The intrinsic etch rate of gold in the aqueous gold etch solution containing $\mathrm{K}_{2} \mathrm{~S}_{2} \mathrm{O}_{3} / \mathrm{K}_{3} \mathrm{Fe}(\mathrm{CN})_{6} / \mathrm{K}_{4} \mathrm{Fe}(\mathrm{CN})_{6}(\mathrm{pH}=14)^{[24]}$ is $\sim 3.5 \mathrm{~nm} \cdot \mathrm{min}^{-1}$ (see Section 4.2 .3 for etch rate determination). At the onset of etching, a reduction rate ( $\mathrm{r}$ ) of the line width of about twice the intrinsic etch rate of gold was estimated from the slope of the plot (Fig. 5.8a, $\mathrm{r}=7.5 \mathrm{~nm} \cdot \mathrm{min}^{-1}$ ). This rate is consistent with the over-etching of the gold taking place at both sides of the etch mask. However, this reduction rate was found to decelerate gradually to about $\mathrm{r}=3.6 \mathrm{~nm} \cdot \mathrm{min}^{-1}$ (Fig. 5.8a). 
As this etch solution is active for at least several hours, a possible explanation for the etching behavior in this typical procedure is that the inward movement of the etching direction underneath the etch mask limits the diffusion of etchants to the etching front. From a detailed study of this etch solution, ${ }^{[24]}$ it is known that the etch rate is sensitive to changes in the concentration of the coordinating ligand (in this case, thiosulfate) and $\mathrm{KOH}$ as, for these electrochemical processes, the oxidation of the gold is the rate-determining step. A reduction in the etch rate by a factor of two, as shown in Fig. 5.8a, was found to correlate to a decrease in the concentration of the coordinating ligand by a factor of ten, or to a decrease in the concentration of $\mathrm{KOH}$ by a factor of five. Since $\mathrm{KOH}$ is in a large excess, the decrease in the concentration of the coordinating ligand at the etching front is the most-probable cause.

By interpolation of the etch curve to the optimal etch time for the $20 \mathrm{~nm}$-thick gold layers $(\sim 7 \mathrm{~min})$, a line width of $\sim 425 \mathrm{~nm}$ was found, which is close to the lateral dimensions of the PS etch mask after $\mathrm{O}_{2}$ plasma treatment $(430 \mathrm{~nm})$. Ultimately, gold patterns with line widths of $\sim 80 \mathrm{~nm}$ have been fabricated with this procedure, although the number of defects in the gold structures increases significantly as the feature sizes approach the grain size of the gold layer. ${ }^{[37]}$

\subsection{Conclusions}

The present results in this chapter demonstrate that capillary force lithography (CFL) is a convenient and inexpensive technique for the fabrication of functional, high-resolution polymer templates with high fidelity and excellent uniformity, without the need of state-of-the-art techniques. The successful application of these templates (i) as masters for stamp fabrication in soft lithography (see Chapter 4), (ii) as molds for NIL, and (iii) as etch masks in the wet-chemical etching of metals illustrates the potential of these templates as tools for high-resolution lithography at the $100 \mathrm{~nm}$ scale. Regarding the ultimate resolution, limitations in the critical dimensions of the resulting patterns are set by the heterogeneity in mass transport inherent to these existing pattern transfer techniques, respectively, alkanethiol inks onto metal surfaces, evaporation of metals onto silicon surfaces and isotropic etching of polymer templates and metals. 
The incorporation of UV-curable mr-L 6000 polymer in the CFL procedure was found to allow the fabrication of high-stability NIL molds by UV crosslinking. A constructive modification of this procedure would constitute crosslinking of the template in situ at the annealing temperature by UV illumination through the (transparent) PDMS stamp after a certain annealing time. Efforts for developing such a direct UV-CFL procedure or combining CFL and photolithography of UV-curable polymers in the fabrication of multilevel structures have not been further undertaken in this study.

The transfer of PDMS onto the template during CFL was shown by XPS analysis to depend on the curing conditions of the PDMS stamps as well as on the type of polymer. For PMMA and low modulus PDMS stamps, the transfer of stamp residues was found to severely compromise the $\mathrm{O}_{2}$ RIE processability of the template in applications that require direct transfer of the template pattern into the underlying substrate (herein, as polymer etch masks for wet-chemical etching of metals). For PS, this was not the case, allowing the residual layer removal and subsequent wet-chemical etching to fabricate $100 \mathrm{~nm}$ gold structures.

The key disadvantage of the current set of edge transfer techniques is that it only allows the fabrication of low-density, straightforward patterns with a feature spacing that is set by the spacing of the original micrometer-size patterns. Although this study has also been dealing only with periodic structures, CFL is capable of fabricating irregular and more complex templates, without substantial modification of the general procedure, through physical self-organization ${ }^{[38]}$ and dewetting, ${ }^{[39,40]}$ and through capillary dynamics. ${ }^{[41]}$ Application of such processes in CFL could provide new opportunities in the fabrication and replication of nanostructures through the combination of top-down and bottom-up approaches.

\subsection{Experimental}

Chemicals and materials: All experiments were carried out in a regular chemical laboratory. Octadecanethiol (ODT), pentaerythritol-tetrakis(3-mercaptopropionate) (PTMP), ethanol, toluene, $\mathrm{NaOH}, \mathrm{Na}_{2} \mathrm{~S}_{2} \mathrm{O}_{3}, \mathrm{~K}_{3} \mathrm{Fe}(\mathrm{CN})_{6}$, and $\mathrm{K}_{4} \mathrm{Fe}(\mathrm{CN})_{6}$ were purchased from commercial sources in the highest available purity and used without further purification. A PDMS prepolymer kit (Sylgard 184 silicone elastomer) 
including PDMS curing agent (Sylgard silicone elastomer curing agent) was purchased from the Dow Corning Corporation. Polystyrene (PS, $\left.M_{w}=240 \mathrm{kDa}\right)$ and poly(methylmethacrylate) (PMMA, $\mathrm{M}_{\mathrm{w}}=38 \mathrm{kDa}$ and $\left.350 \mathrm{kDa}\right)$ and were purchased from Acros Organics; mr-L 6000 was donated by Microresist Technology GmbH, Germany. $100 \mathrm{~mm}$ p-type OSP silicon wafers were purchased from Okmetic and the $20 \mathrm{~nm}$ gold-on-silicon wafers from Ssens BV (Hengelo, The Netherlands).

Stamp preparation: Stamps were fabricated by casting a 10:1 (v/v) mixture of PDMS and curing agent (Sylgard 184, Dow Corning) against a relief pattern of interest (i.e., silicon master or polymer template). After curing for $1-18 \mathrm{~h}$ at $60^{\circ} \mathrm{C}$, the stamps were released at this curing temperature to avoid buildup of tension due to thermal shrinkage. ${ }^{[42]}$ For CFL, the thickness of these PDMS stamps was about $1 \mathrm{~mm}$ to ensure sufficient flexibility.

Polymer template preparation: Silicon substrates of $2 \mathrm{~cm} \times 2 \mathrm{~cm}$ were cleaned by ultrasonic treatment in acetone for $15 \mathrm{~min}$, and dried in a continuous stream of nitrogen. Polymer solutions were applied onto clean silicon substrates by filtering through a syringe filter with a $0.2 \mu \mathrm{m}$ PTFE membrane (Acrodisc CR 25mm). Spincoating was done at $3000 \mathrm{rpm}$ for $30 \mathrm{~s}$ in order to obtain polymer layers of about $150 \mathrm{~nm}$ in thickness, followed by a prebake at $100-120{ }^{\circ} \mathrm{C}$ for $10 \mathrm{~min}$ to remove residual solvent. The stamp and the substrate were equilibrated at the annealing temperature for $30 \mathrm{~min}$ and contact was applied manually with minimal delay to prevent any dust interference. The stamp plus substrate were left in the oven at about $40{ }^{\circ} \mathrm{C}$ above the glass-transition temperature of the respective polymer for $2-20 \mathrm{~h}$. After cooling for $5 \mathrm{~s}$ at room temperature, the PDMS stamp was released from the polymer substrate.

Nanoimprint lithography: Prior to imprinting, crosslinking of the mr-L 6000 polymer template was induced by flood-exposure of the template under a mask aligner (Karl Süss mask aligner MA55) at a dose of $360 \mathrm{~mJ} \cdot \mathrm{cm}^{-2}$ (at an intensity of $9 \mathrm{~mW} \cdot \mathrm{cm}^{-2}$ ) and post-exposure baking at $100{ }^{\circ} \mathrm{C}$ for $10 \mathrm{~min}$. To function as a NIL mold, additional thermal and mechanical stability was achieved by flood-exposure of this template for $10 \mathrm{~J} \cdot \mathrm{cm}^{-2}$ and hard baking at $120^{\circ} \mathrm{C}$ for $10 \mathrm{~min}$.

For imprinting the mold pattern into a thermoplastic polymer (PMMA, $\mathrm{M}_{\mathrm{w}}=350 \mathrm{kDa}$ ), the second-generation mold plus polymer substrate were placed in 
between the plates of a commercial hydraulic press (Specac, 15 tons manual press equipped with electrical heating plates and a temperature controller) and heated to the imprint temperature of $180{ }^{\circ} \mathrm{C}$ (heating rate $\sim 35^{\circ} \mathrm{C} \cdot \mathrm{min}^{-1}$ ). Upon reaching this imprint temperature, an imprint pressure of 100 bar was applied to pattern the PMMA layer. After 1 min imprinting at $180{ }^{\circ} \mathrm{C}$ and 100 bar, the stack was cooled (cooling rate $\sim 20{ }^{\circ} \mathrm{C} \cdot \mathrm{min}^{-1}$ ) to $106^{\circ} \mathrm{C}$ prior to pressure reduction and manual cleavage of the mold from the polymer substrate with a scalpel. The total process cycle (i.e., heating, 1 min imprinting, and cooling) took about 7 min.

Metal evaporation was performed on a commercial evaporation system (Balzers BAK600, E-gun evaporating system). Prior to evaporation, the imprints were exposed to $\mathrm{O}_{2} \mathrm{RIE}\left(50 \mathrm{mTorr}, 50 \mathrm{~W}, 20 \mathrm{sccm} \mathrm{O}_{2}\right.$ ) for 15-20 s. The metals were evaporated on the imprint in the following order: chromium $\left(2-3 \mathrm{~nm}, \sim 1 \AA \cdot \mathrm{s}^{-1}\right.$ at $\left.2 \cdot 10^{-6} \mathrm{mbar}\right)$; gold (10 nm, $\sim \AA \AA \mathrm{s}^{-1}$ at $\left.3 \cdot 10^{-6} \mathrm{mbar}\right)$. Finally, the substrates were sonicated in acetone at $40{ }^{\circ} \mathrm{C}$ for $15 \mathrm{~min}$.

Undercutting by isotropic wet-chemical etching: The PS templates were exposed to $\mathrm{O}_{2}$ RIE ( $50 \mathrm{~m}$ Torr, $50 \mathrm{~W}, 20 \mathrm{sccm} \mathrm{O}_{2}$ ) for $120 \mathrm{~s}$ in order to remove the residual layer. The resulting oxide layer on the gold was reduced by leaving the substrates in absolute ethanol for $30 \mathrm{~min} .{ }^{[43]}$ Subsequently, etching of the underlying gold was achieved by immersing the templates in an aqueous solution of $1.0 \mathrm{M} \mathrm{NaOH}, 0.1 \mathrm{M} \mathrm{Na}_{2} \mathrm{~S}_{2} \mathrm{O}_{3}$, $0.01 \mathrm{M} \mathrm{K}_{3} \mathrm{Fe}(\mathrm{CN})_{6}$, and $0.001 \mathrm{M} \mathrm{K}_{4} \mathrm{Fe}(\mathrm{CN})_{6}$ for various times at room temperature. [CAUTION: potassium ferricyanide is light sensitive. Photodecomposition releases products that can contain free cyanide. Potassium ferricyanide is also incompatible with acids and liberates $\mathrm{HCN}$ ]

Characterization: Polymer film thicknesses were determined with a Plasmos SD 2002 ellipsometer operating at a wavelength of $632.8 \mathrm{~nm}$.

AFM imaging was carried out with a NanoScope III Multimode AFM (Digital Instruments, Santa Barbara, CA, USA) operating in contact mode using $\mathrm{Si}_{3} \mathrm{~N}_{4}$ cantilevers (Nanoprobes, Digital Instruments) with an approximate spring constant of $0.32 \mathrm{~N} \cdot \mathrm{m}^{-1}$. For height measurements, the scanner was calibrated in $\mathrm{z}$-direction using a calibration standard with known step height. 
Low-resolution SEM imaging was carried out with a JEOL JSM-5610 SEM operating in secondary electron detection. High-resolution SEM imaging was carried out with a LEO Gemini 1550 FEG-SEM.

XPS analyses were carried out with a Physical Electronics Quantum2000 equipped with an Al K Alpha monochromatic excitation source (source energy = $1486.7 \mathrm{eV}$, take-off angle set to $45^{\circ}$ ), a spherical sector analyzer and a multichannel plate detector (16 detector elements). For the survey scan (pass energy of $224 \mathrm{eV}$ ), the $\mathrm{X}$-ray beam was set to $25 \mathrm{~W}$ per $100 \mu \mathrm{m}$ to scan a total area of $700 \mu \mathrm{m} \times 300 \mu \mathrm{m}$; for element scans (pass energy of $224 \mathrm{eV}$ ), the X-ray beam was set to $25 \mathrm{~W}$ per $100 \mu \mathrm{m}$ to scan a total scan area of $700 \mu \mathrm{m} \times 300 \mu \mathrm{m}\left(298 \mathrm{~K}\right.$ and $1-3 \cdot 10^{-8}$ Torr). The sensitivity factors used for $\mathrm{C}, \mathrm{O}$, and $\mathrm{Si}$ for calculating the atomic concentration are 0.314, 0.733, and 0.368 , respectively. The hydrocarbon $\mathrm{C}(1 \mathrm{~s})$ signal at $284.8 \mathrm{eV}$ was used as a reference to correct for surface charging.

\subsection{References and notes}

[1] S. J. Holmes, P. H. Michel, M. C. Hakey, IBM J. Res. Dev. 1997, 41, 7.

[2] J. P. Silverman, J. Vac. Sci. Technol. B 1997, 15, 2117.

[3] M. A. McCord, J. Vac. Sci. Technol. B 1997, 15, 2125.

[4] U. S. Tandon, Vacuum 1992,43, 241.

[5] Y. Xia, G. M. Whitesides, Angew. Chem. Int. Ed. 1998, 37, 550.

[6] S. Y. Chou, P. R. Krauss, P. J. Renstrom, Science 1996, 272, 85.

[7] (a) J. A. Rogers, K. E. Paul, R. J. Jackman, G. M. Whitesides, Appl. Phys. Lett. 1997, 70, 2658. (b) T. W. Odom, V. R. Thalladi, J. C. Love, G. M. Whitesides, J. Am. Chem. Soc. 2002, $124,12112$.

[8] K. E. Paul, T. L. Breen, J. Aizenberg, G. M. Whitesides, Appl. Phys. Lett. 1998, 73, 2893.

[9] J. C. Love, K. E. Paul, G. M. Whitesides, Adv. Mater. 2001, 13, 604.

[10] (a) J. Aizenberg, A. J. Black, G. M. Whitesides, Nature 1998, 394, 868. (b) A. J. Black, K. E. Paul, J. Aizenberg, G. M. Whitesides, J. Am. Chem. Soc. 1999, 121, 8356.

[11] M. Geissler, J. M. McLellan, Y. Xia, Nano Lett. 2005, 5, 31.

[12] J. Haneveld, E. Berenschot, P. A. Maury, M. Elwenspoek, J. Micromech. Microeng. 2006, 16, S24.

[13] K.-H. Chung, W. Y. Choi, S.-K. Sung, D. H. Kim, J. D. Lee, B.-G. Park, J. Vac. Sci. Technol. B 2003, 21, 1491. 
[14] K. Y. Suh, H. H. Lee, Adv. Funct. Mater. 2002, 12, 405.

[15] K. Y. Suh, Y. S. Kim, H. H. Lee, Adv. Mater. 2001, 13, 1386.

[16] C. M. Bruinink, M. Péter, M. de Boer, L. Kuipers, J. Huskens, D. N. Reinhoudt, Adv. Mater. 2004, 16, 1086.

[17] K. Y. Suh, S. Chu, H.H. Lee, J. Micromech. Microeng. 2005, 14, 1185.

[18] K. Y. Suh, P. J. Yoo, H.H. Lee, Macromolecules 2002, 35, 4414.

[19] M. Wissen, H. Schultz, N. Bogdanski, H.-C. Scheer, Y. Hirai, H. Kikuta, G. Ahrens, F. Reuther, K. Pfeiffer, Microelecron. Eng. 2004, 73-74, 184.

[20] R. W. Jaszewski, H. Schift, B. Schnyder, A. Schneuwly, P. Gröning, Appl. Surf. Sci. 1999, $143,301$.

[21] M. Bender, M. Otto, B. Hadam, B. Spangenberg, H. Kurz, Microelectron. Eng. 2002, 61-62, 407.

[22] The presence of fluorine-containing additives that are well-known for lowering the surface energy of any surface was excluded by XPS analysis of mr-L 6000. Although no specific elements were detected by XPS analysis that could confirm the presence of other types of surfactants in this commercial resist, the product specifications affirm the use of such additives in mr-L 6000 (i.e., 'surface smoothers') in order to increase the surface smoothness of resist layers in spin coating. Furthermore, the low pattern density can account in part for the excellent properties of the final mr-L 6000 templates as NIL molds.

[23] B. Faircloth, H. Rohrs, R. Tiberio, R. Ruoff, R. R. Krchnavek, J. Vac. Sci. Technol. B 2000, 18, 1866.

[24] Y. Xia, X.-M. Zhao, E. Kim, G. M. Whitesides, Chem. Mater. 1995, 7, 2332.

[25] S.Y. Chou, P.R. Krauss, W. Zhang, L. Guo, L. Zhuang, J. Vac. Sci. Technol. B 1997, 15, 2897.

[26] I. Manners, Chem. Commun. 1999, 10, 857.

[27] I. Korczagin, S. Golze, M. A. Hempenius, G. J. Vancso, Chem. Mater. 2003, 15, 3663.

[28] R. D. Miller, G. M. Wallraff, Adv. Mater. Opt. Electron. 1994, 4, 95.

[29] G. Beamson, D. Briggs, High resolution XPS of organic polymers: the Scienta ESCA300 database, John Wiley \& Sons, Chichester, UK 1992.

[30] AFM height analysis demonstrates that $80 \mathrm{~nm}$ of the $150 \mathrm{~nm}$ thick residual layer is still present in the contact areas after $100 \mathrm{~s} \mathrm{O}_{2}$ RIE. Calculations suggest that the AFM height profile in Fig. 5.6a is the result of an etch rate reduction of at least $60 \%$ (from $1.9 \mathrm{~nm}^{\cdot-1} \mathrm{~s}^{-1}$ to $\left.0.7 \mathrm{~nm} \cdot \mathrm{s}^{-1}\right)$.

[31] K. Glasmäster, J. Gold, A.-S. Andersson, D. S. Sutherland, B. Kasemo, Langmuir 2003, 19, 5475 . 
[32] H. Gokan, Y. Saotome, K. Saigo, F. Watanabe, Y. Ohnishi, in Polymers for High Technology, Electronics, and Photonics (Eds: S. R. Turner, M. J. Bowden), ACS Symposium Series, American Chemical Society, Washington, DC 1986, pp. 358-368.

[33] J. F. Moulder, W. F. Stickle, P. E. Sobol, K. Bomben, Handbook of X-ray Photoelectron Spectroscopy (Ed: J. Chastain), Perkin-Elmer Corporation, Eden Prairie, MN 1992.

[34] V. Z.-H. Chan, E. L. Thomas, J. Frommer, D. Sampson, R. Campbell, D. Miller, C. Hawker, V. Lee, R. D. Miller, Chem. Mater. 1998, 10, 3895.

[35] W. Kern, C. A. Deckert, in Thin Film Processes II (Eds: J. L. Vossen, W. Kern), Academic, Boston, MA 1978.

[36] At these dimensions, the standard deviation $(\sigma)$ in the line width of the gold rings is $\sim 30 \mathrm{~nm}$ : about $50 \%$ of the ring in Fig. 5.7c (and Fig. 5.8e for close-up SEM image) has lateral dimensions in the sub-100 $\mathrm{nm}$ range $(>70 \mathrm{~nm})$.

[37] At these dimensions, the grain size of the metal layer is known the affect the ultimate resolution. For our gold layers, a grain size distribution of 30-60 nm was determined by AFM.

[38] K. Y. Suh, H. H. Lee, Adv. Mater. 2002, 14, 346.

[39] K. Y. Suh, J. Park, H. H. Lee, J. Chem. Phys. B 2002, 116, 7714.

[40] C. Luo, R. Xing, Y. Han, Surf. Sci. 2004, 552, 139.

[41] K. Y. Suh, H. H. Lee, J. Micromech. Microeng. 2005, 15, 400.

[42] H. Schmid, B. Michel, Macromolecules 2000, 33, 3042.

[43] H. Ron, I. Rubinstein, Langmuir 1994, 10, 4566. 


\section{Accurate pattern inversion and pattern transfer techniques for nanolithography}

This chapter describes the local oxidation of silicon (LOCOS) as a means to convert resist patterns into inverse patterns of thermal $\mathrm{SiO}_{2}$ by incorporation of ultrathin functional transfer layers into current fabrication processes. The high transfer accuracy and the excellent feature definition throughout the entire inversion procedure demonstrate the potential of this fabrication procedure in nanolithography. Accurate sub-500 $\mathrm{nm}$ deep silicon etching at high anisotropy and high etch selectivity with respect to ultrathin etch masks of thermal $\mathrm{SiO}_{2}$ illustrates the merits of a systematic etch procedure.

\subsection{Introduction}

The development of micro- and nanolithography in terms of ultimate resolution, process control and economic performance generally depends upon the continuous investments in research and process development. The technological advancements in high-resolution lithography and post-processing limit the applicability of conventional resist systems due to the stringent requirements on the properties of the resist with respect to exposure sensitivity, contrast, resolution and (plasma) etch resistance. An 
interesting development during the last decades is the engineering of many novel resists and/or composite resist systems into the lithographic fabrication process to overcome the problems of conventional resist systems. ${ }^{[1,2]}$ Of technological importance is the invention of multilayer resist systems, allowing a decoupling of the imaging and resolution aspects of pattern transfer of high-resolution lithographic techniques (deepUV (DUV) and extreme-UV (EUV) photolithography, scanning electron beam and ion beam lithography). Generally, a multilayer resist system comprises a thin radiation-sensitive top layer (for resolution purposes) plus a thin intermediate inorganic layer (for plasma etch resistance), or a radiation-sensitive etch-resistant resist, in combination with a thick organic base layer (for planarization and feature aspect ratio purposes). After exposure and development of the high-resolution features in the top layer, successive plasma etch steps of the intermediate and base layer are necessary to transfer the final pattern into the substrate by any additive (e.g., metal liftoff) or subtractive (e.g., reactive ion etching) technique.

As a non-photolithographic technique, nanoimprint lithography (NIL) ${ }^{[3]}$ is not prone to many of the factors that limit the resolution of these high-resolution lithographic techniques such as diffraction, scattering and interference, backscattering from a substrate, or from the chemistry of the resist and the developer. Actually, NIL is the most promising technique for becoming the next-generation high-resolution lithographic tool of the semiconductor industry owing to its wafer-scale patterning capability at sub-10 $\mathrm{nm}$ resolution. ${ }^{[4]}$ The reintroduction of multilayer resist systems in NIL ${ }^{[5,6]} 25$ years after its invention seems surprising, however, this has been done for two main reasons: (i) to generate a proper undercut to facilitate metal lift-off, and (ii) to increase the aspect ratio of the polymer features. However, the advantage of using multilayer resist systems in their present form comes at the expense of process complexity arising from the increase in the number of plasma processing steps. Additionally, the accurate transfer of high-resolution features into silicon necessitate the exact formulation of an anisotropic etch procedure capable of etching silicon at a low etch rate and at a high etch selectivity with respect to mask materials.

This chapter demonstrates the successful implementation of the local oxidation of silicon $(\mathrm{LOCOS})^{[7]}$ and ultrathin functional transfer layers to convert resist patterns into inverse patterns of thermal silicon oxide $\left(\mathrm{SiO}_{2}\right)$ and a systematic procedure for the 
anisotropic etching of silicon. It is of great importance that the entire lithographic procedure is applicable to primary nanolithographic techniques as NIL (see Chapter 7, fabrication of 2D-photonic crystal waveguides (2D PhCWGs) on silicon-on-insulator (SOI)) and compatible with the conventional semiconductor device fabrication technology. The transfer accuracy and feature definition during the successive transfer steps are the most important output factors in the process evaluation by highresolution scanning electron microscopy (SEM). Therefore, emphasis was put on the systematic use of design rules for the present lithographic procedures in terms of pattern transfer fidelity, etch rate and selectivity.

\subsection{Results and discussion}

\subsubsection{LOCOS for pattern inversion into thermal $\mathrm{SiO}_{2}$}

LOCOS is a well-known isolation technique ${ }^{[8]}$ in semiconductor device fabrication technology, patented by Philips in the 1960s after the observation that the oxidation rate of silicon is much higher than that of silicon nitride. Conventional LOCOS concerns the selective oxidation of silicon using thin silicon nitride layers as oxidation masks and is compatible with many existing technologies. The resulting field oxides serve as masks during impurity diffusion (i.e., doping) of the active areas in device fabrication. The reduction in dimension losses in LOCOS to field oxides has been the biggest challenge for process engineers to scale LOCOS into the sub-micrometer regime due to the lateral oxide encroachment under the silicon nitride mask and field oxide thinning, ${ }^{[9]}$ resulting in the development of many LOCOS type isolation techniques during the last decades. ${ }^{[10]}$

\subsubsection{Design rules for the transfer layers}

By setting proper design rules, conventional LOCOS can still be applicable as a technique to convert resist patterns into inverse patterns of thermal $\mathrm{SiO}_{2}$ on the nanometer scale. Scheme 6.1 illustrates this LOCOS pattern inversion procedure. 


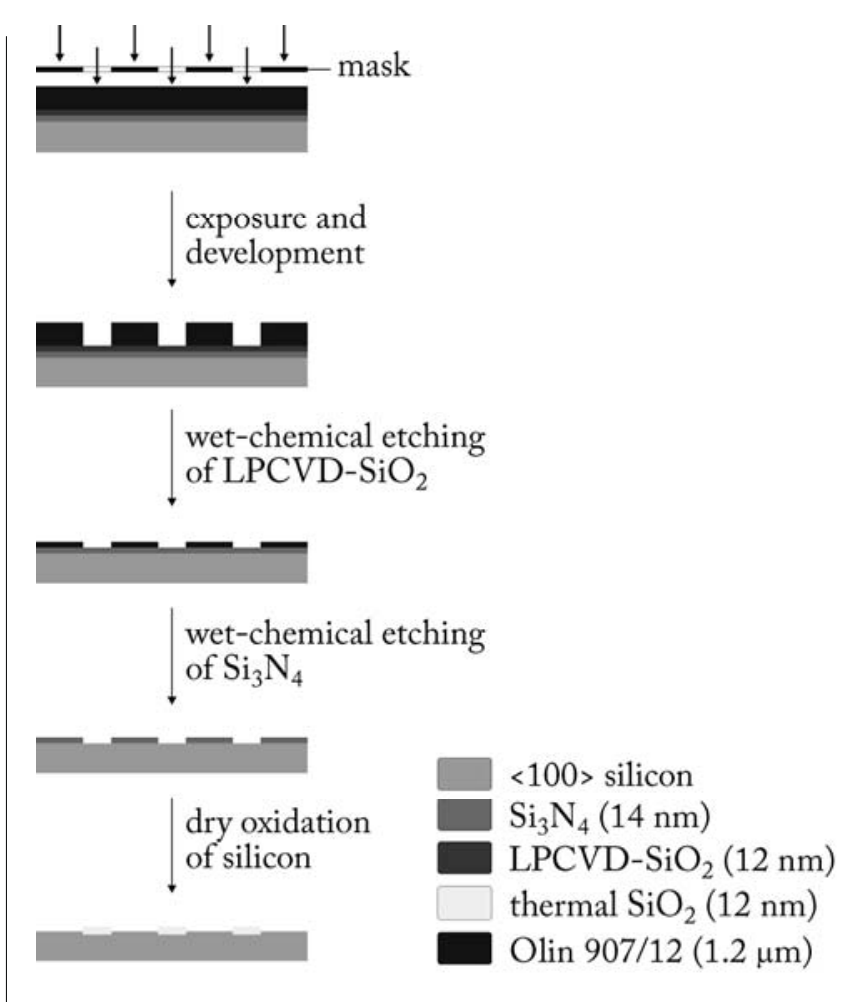

Scheme 6.1 Schematic representation of the LOCOS pattern inversion procedure in 3 steps from an arbitrary resist pattern: (1) wet-chemical etching of $\mathrm{LPCVD}-\mathrm{SiO}_{2}$ in $1 \% \mathrm{HF}$, (2) wet-chemical etching of $\mathrm{Si}_{3} \mathrm{~N}_{4}$ in $85 \% \mathrm{H}_{3} \mathrm{PO}_{4}\left(180{ }^{\circ} \mathrm{C}\right)$, and (3) dry oxidation of silicon.

A successive deposition of ultrathin layers of stoichiometric silicon nitride $\left(\mathrm{Si}_{3} \mathrm{~N}_{4}\right)$ and $\mathrm{SiO}_{2}$ by low-pressure chemical vapor deposition (LPCVD) makes any substrate compatible for this LOCOS pattern inversion procedure. These two layers have the following function:

- LPCVD-SiO 2 layer: sacrificial transfer layer to convert resist patterns into more etch-resistant inorganic patterns, as resists are incompatible with the strong oxidation conditions necessary for direct patterning of the $\mathrm{Si}_{3} \mathrm{~N}_{4}$ layer;

- $\mathrm{Si}_{3} \mathrm{~N}_{4}$ layer: oxidation transfer layer to invert the patterns into thermal $\mathrm{SiO}_{2}$.

The design rules set limits to the process parameters in terms of layer thicknesses and etch selectivities of the different materials (i.e., $\mathrm{LPCVD}-\mathrm{SiO}_{2}, \mathrm{Si}_{3} \mathrm{~N}_{4}$ and thermal $\mathrm{SiO}_{2}$ ), and oxidation resistance of the $\mathrm{Si}_{3} \mathrm{~N}_{4}$. The successive pattern transfer and final inversion to thermal $\mathrm{SiO}_{2}$ requires the use of ultrathin layers $(\sim 10 \mathrm{~nm})$ in order to 
minimize dimension losses due to the isotropic nature of wet-chemical etching and lateral oxide encroachment, respectively. Investigation of the etch rates of the different materials in $1 \%$ hydrogen fluoride $(\mathrm{HF})$ and in $85 \%$ phosphoric acid $\left(\mathrm{H}_{3} \mathrm{PO}_{4}\right)$ at $180{ }^{\circ} \mathrm{C}$ was necessary in order to optimize the inversion procedure in terms of etch selectivity. The intrinsic etch rates of $\mathrm{LPCVD}-\mathrm{SiO}_{2}, \mathrm{Si}_{3} \mathrm{~N}_{4}$ and thermal $\mathrm{SiO}_{2}$ in $1 \% \mathrm{HF}$ and in $85 \% \mathrm{H}_{3} \mathrm{PO}_{4}$ at $180{ }^{\circ} \mathrm{C}$ are shown in Table 6.1.

Table 6.1. Intrinsic etch rates of $\mathrm{LPCVD}-\mathrm{SiO}_{2}, \mathrm{Si}_{3} \mathrm{~N}_{4}$, and thermal $\mathrm{SiO}_{2}$ in $1 \% \mathrm{HF}$ at room temperature and in $85 \% \mathrm{H}_{3} \mathrm{PO}_{4}$ at $180{ }^{\circ} \mathrm{C}$, including the etch rates of the materials after 60 min thermal annealing under dry nitrogen at three different temperatures.

\begin{tabular}{|c|c|c|c|c|c|c|c|c|}
\hline & \multicolumn{8}{|c|}{ Etch rates $\left[\mathrm{nm} \cdot \mathrm{min}^{-1}\right]^{[11]}$} \\
\hline & \multicolumn{2}{|c|}{ intrinsic } & \multicolumn{2}{|c|}{$800{ }^{\circ} \mathrm{C}$ annealing } & \multicolumn{2}{|c|}{$900^{\circ} \mathrm{C}$ annealing } & \multicolumn{2}{|c|}{$1050{ }^{\circ} \mathrm{C}$ annealing } \\
\hline & $\mathrm{HF}$ & $\mathrm{H}_{3} \mathrm{PO}_{4}$ & $\mathrm{HF}$ & $\mathrm{H}_{3} \mathrm{PO}_{4}$ & $\mathrm{HF}$ & $\mathrm{H}_{3} \mathrm{PO}_{4}$ & $\mathrm{HF}$ & $\mathrm{H}_{3} \mathrm{PO}_{4}$ \\
\hline LPCVD- $\mathrm{SiO}_{2}$ & 31 & 2.9 & 23 & 1.1 & 12 & 0.55 & 4.6 & 0.26 \\
\hline $\mathrm{Si}_{3} \mathrm{~N}_{4}$ & 0.30 & 4.2 & 0.29 & 4.1 & 0.24 & 4.2 & 0.15 & 3.9 \\
\hline thermal $\mathrm{SiO}_{2}$ & 4.2 & 0.25 & - & - & - & - & - & - \\
\hline $\mathrm{Si}$ & - & $0.3^{[12]}$ & - & - & - & - & - & - \\
\hline
\end{tabular}

Etch selectivity of the mask material over the target material is important as it allows over-etching to ensure complete removal of the target material in a particular etch solution and complete removal of the mask material afterwards in another etch solution. ${ }^{[13]}$ The etch conditions were set to over-etch the LPCVD-SiO layers by $50 \%$ with respect to the (theoretical) etch time. As the pattern inversion procedure requires two consecutive etch steps, including $\mathrm{LPCVD}-\mathrm{SiO}_{2}$ as the target material and mask material, the etch selectivities of $\mathrm{LPCVD}-\mathrm{SiO}_{2}$ to resist (in $1 \% \mathrm{HF}$ ) and of $\mathrm{Si}_{3} \mathrm{~N}_{4}$ to $\mathrm{LPCVD}-\mathrm{SiO}_{2}\left(\right.$ in $85 \% \mathrm{H}_{3} \mathrm{PO}_{4}$ at $180{ }^{\circ} \mathrm{C}$ ) must therefore be taken into consideration at the same time.

The intrinsic etch selectivity of $\mathrm{Si}_{3} \mathrm{~N}_{4}$ over LPCVD- $\mathrm{SiO}_{2}$ in $85 \% \mathrm{H}_{3} \mathrm{PO}_{4}$ at $180{ }^{\circ} \mathrm{C}$ (Table 6.1, selectivity of 1.4) was insufficient to allow substantial over-etching due to a relatively large intrinsic $\mathrm{LPCVD}-\mathrm{SiO}_{2}$ etch rate. Previous studies on the material 
properties of $\mathrm{LPCVD}-\mathrm{SiO}_{2}$ have shown that high-temperature annealing can cause a strong reduction of the $\mathrm{LPCVD}-\mathrm{SiO}_{2}$ etch rate due to reduction of the layer porosity by desorption of internal water and structural rearrangements within the LPCVD$\mathrm{SiO}_{2}$ layer as shown by infrared spectroscopy. ${ }^{[14]}$ The effect of thermal annealing on the etch rates of $\mathrm{LPCVD}-\mathrm{SiO}_{2}\left(\right.$ and $\mathrm{Si}_{3} \mathrm{~N}_{4}$ ) in the two etch solutions is also shown in Table 6.1 (and Fig. 6.1a) at three different annealing temperatures.

(a)

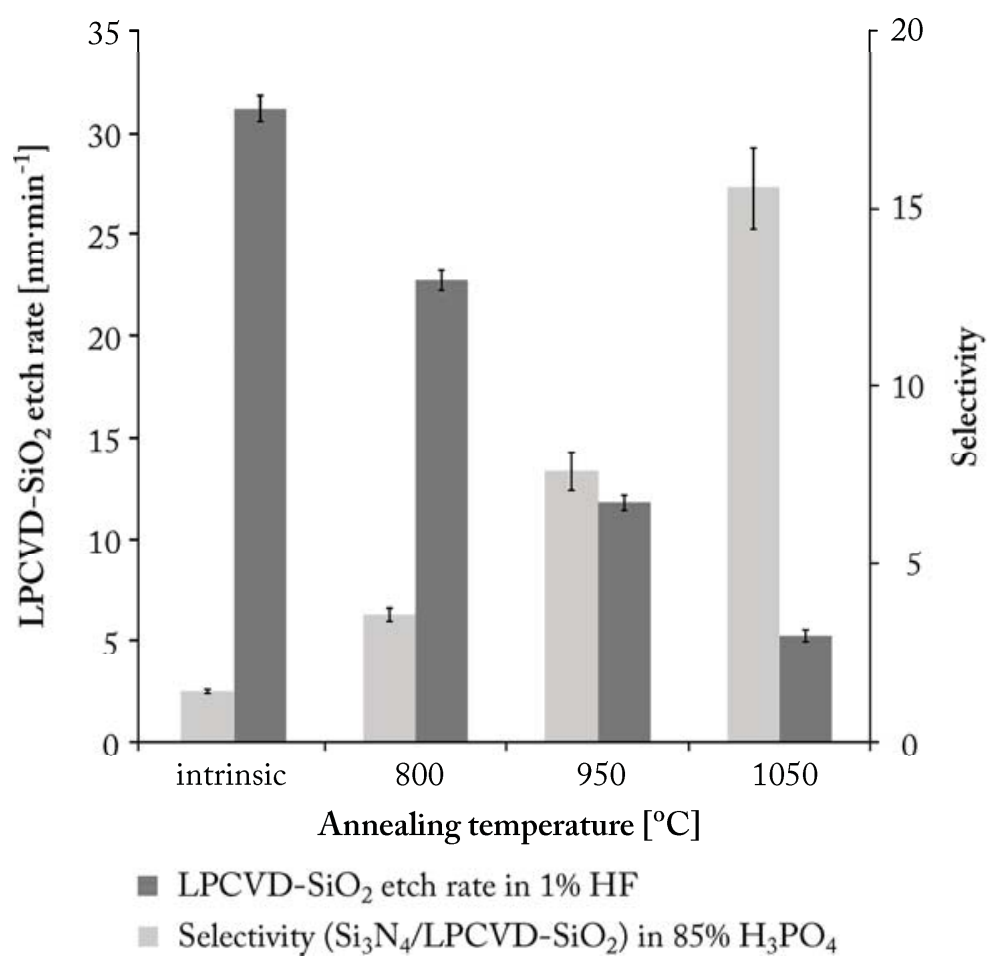

(b)

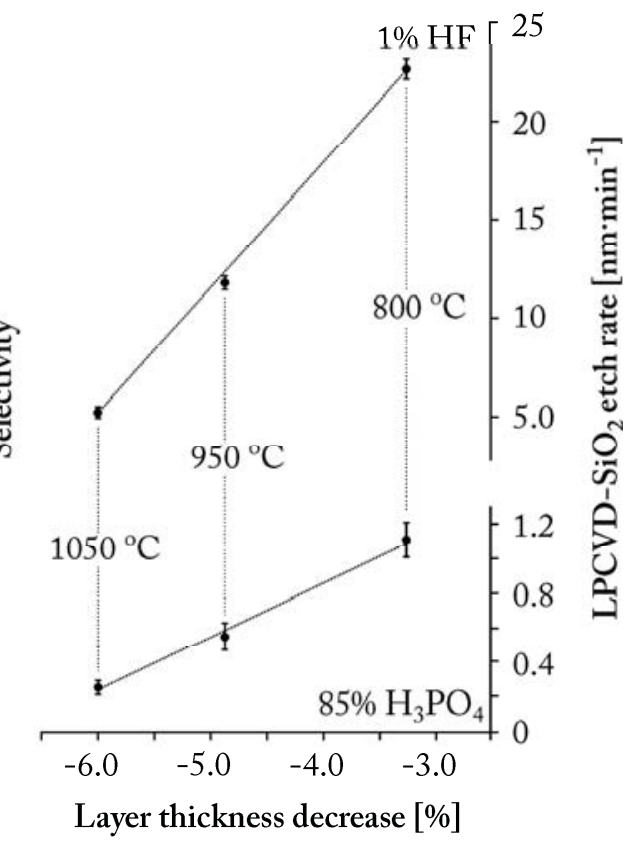

Figure 6.1 Etch rate dependence of LPCVD-SiO ${ }_{2}$ in $1 \% \mathrm{HF}$ and $85 \% \mathrm{H}_{3} \mathrm{PO}_{4}\left(180{ }^{\circ} \mathrm{C}\right)$ as a function of the annealing temperature (a) and the corresponding decrease in layer thickness (b).

The corresponding decrease in thickness of the $\mathrm{LPCVD}-\mathrm{SiO}_{2}$ layers upon thermal annealing at 800,900 and $1050^{\circ} \mathrm{C}$ was found to conform to a linear correlation with the resulting $\mathrm{LPCVD} \mathrm{SiO}_{2}$ etch rate in both etch solutions (Fig. 6.1b). In accordance with the literature, the $\mathrm{LPCVD} \mathrm{SiO}_{2}$ etch rate approximates the etch rate of thermal $\mathrm{SiO}_{2}$ at high-temperature thermal annealing (Table 6.1, $\mathrm{T}_{\text {anneal }}=1050{ }^{\circ} \mathrm{C}$ ). The results in Table 6.1 illustrate that the etch selectivity of $\mathrm{Si}_{3} \mathrm{~N}_{4}$ over $\mathrm{LPCVD}-\mathrm{SiO}_{2}$ in $85 \% \mathrm{H}_{3} \mathrm{PO}_{4}\left(180^{\circ} \mathrm{C}\right)$, as also shown in Fig. 6.1a, is in fact only susceptible to thermal annealing by the LPCVD-SiO 
LPCVD- $\mathrm{SiO}_{2}$ in $85 \% \mathrm{H}_{3} \mathrm{PO}_{4}\left(180{ }^{\circ} \mathrm{C}\right)$ serves the over-etching process condition at any of the thermal annealing temperatures. As a result, the etch rate of $\mathrm{LPCVD}-\mathrm{SiO}_{2}$ in $1 \% \mathrm{HF}$ was the decisive factor in the engineering process of the present lithographic procedure. It was found to decrease with annealing temperature (Fig. 6.1a). Thermal annealing at $800{ }^{\circ} \mathrm{C}$ was chosen after taking into account that the ultimate aim is to exploit this novel inversion process in thermal NIL of 2D PhCWGs on SOI, i.e., the resulting $\mathrm{LPCVD}-\mathrm{SiO}_{2}$ etch rate in $1 \% \mathrm{HF}$ has to tolerate patterning of this transfer layer within the resistance time (< 1-2 min) of sub-100 nm thick polymer imprints to $1 \%$ HF (see Chapter 7). With the etch rate and selectivity set, the last parameter that determines the final layer thickness of the transfer layer is the oxidation resistance of $\mathrm{Si}_{3} \mathrm{~N}_{4}$ in dry oxidation conditions. Fig. 6.2 illustrates the etch plot of a $\mathrm{Si}_{3} \mathrm{~N}_{4}$ layer in $1 \% \mathrm{HF}$ after a $10 \mathrm{~h}$ oxidation run at $950{ }^{\circ} \mathrm{C}$.

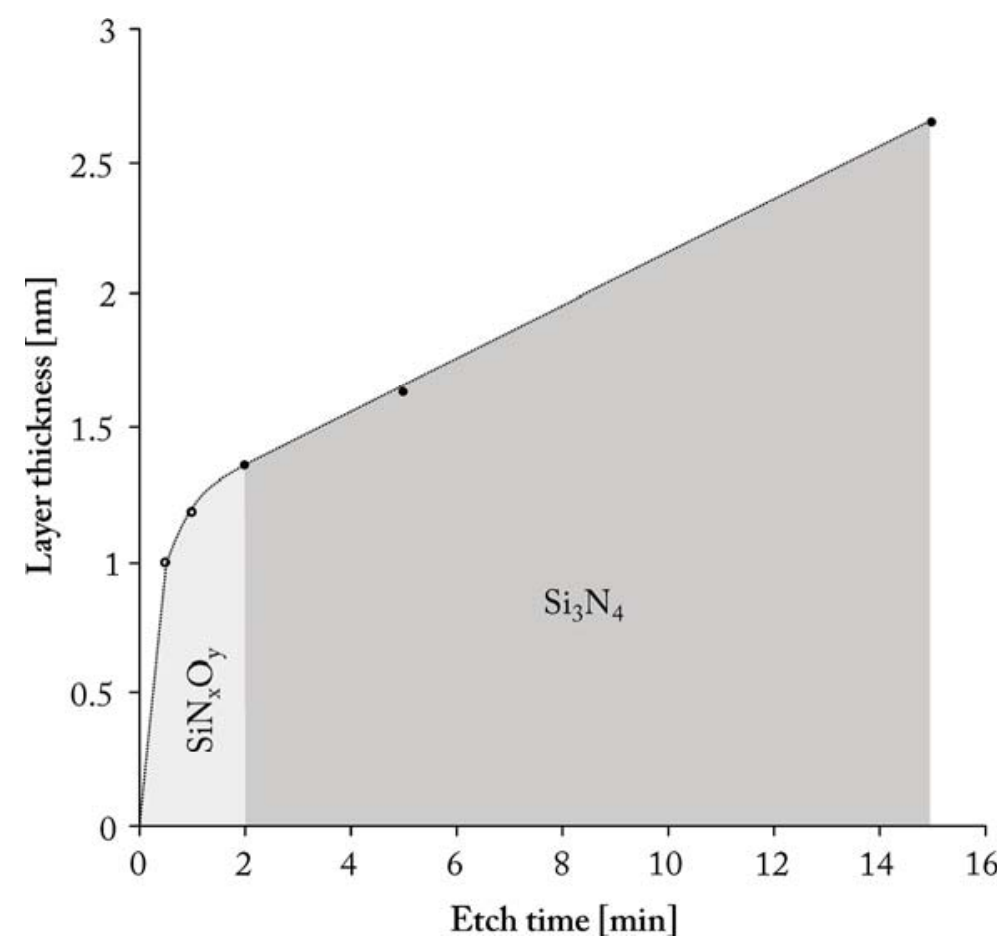

Figure 6.2 Etch rate dependence of a $\mathrm{Si}_{3} \mathrm{~N}_{4}$ layer in $1 \% \mathrm{HF}$ after a ten hour oxidation run at $950{ }^{\circ} \mathrm{C}$. All data points come from ellipsometer measurements on the same substrate after successive etching in $1 \% \mathrm{HF}$. For the measurements, due to the heterogeneous nature of the layer after oxidation, the refractive-index of $\mathrm{Si}_{3} \mathrm{~N}_{4}$ $(n=2.00)$ was taken to estimate (open dots) or determine (solid dots) the total layer thickness.

The partial conversion of $\mathrm{Si}_{3} \mathrm{~N}_{4}$ into silicon oxynitride $\left(\mathrm{SiN}_{\mathrm{x}} \mathrm{O}_{\mathrm{y}}\right)$ under dry oxidation conditions is known in literature. ${ }^{[15,16]}$ Estimation of the oxidation resistance is feasible 
due to the large drop in the etch rate in $1 \% \mathrm{HF}$ due to the heterogeneous nature of the layer after oxidation. The intrinsic etch rate of $\sim 2 \mathrm{~nm} \cdot \mathrm{min}^{-1}$ drops by a factor of 20 to about $0.1 \mathrm{~nm} \cdot \mathrm{min}^{-1}$, indicating entry to the core $\mathrm{Si}_{3} \mathrm{~N}_{4}$ (Fig. 6.2).

Ellipsometer measurements on a second substrate were carried out to estimate the thickness of the top $\left(\mathrm{SiN}_{\mathrm{x}} \mathrm{O}_{\mathrm{y}}\right)$ layer using a two-layer model with the thickness of the core $\mathrm{Si}_{3} \mathrm{~N}_{4}$ and the refractive-indices of $\mathrm{Si}_{3} \mathrm{~N}_{4}(\mathrm{n}=2.00)$ and $\mathrm{SiO}_{2}(\mathrm{n}=1.465)$ as input parameters. The top layer was found to range from $1.2 \mathrm{~nm}$ to $1.4 \mathrm{~nm}$, indicating the lower limit of the thickness of the $\mathrm{Si}_{3} \mathrm{~N}_{4}$ layer to resist oxidation. However, the present LPCVD system limits the layer thickness of the $\mathrm{Si}_{3} \mathrm{~N}_{4}$ layer to about $12 \mathrm{~nm}{ }^{[17]}$ The conditions for the low-pressure chemical vapor deposition (LPCVD) of the two transfer layers were set to result in a layer thickness of about $12 \mathrm{~nm}$.

\subsubsection{Pattern inversion of resist patterns into thermal $\mathrm{SiO}_{2}$}

After an introductory standard cleaning procedure of the 4-inch silicon wafers and native oxide removal in $1 \% \mathrm{HF}$, immediate deposition of very thin layers of $\mathrm{Si}_{3} \mathrm{~N}_{4}$ $(14 \mathrm{~nm})$ and LPCVD-SiO $2(12 \mathrm{~nm})$ was performed by LPCVD in order to prevent re-oxidation of the silicon to native oxide. Prior to processing the substrate by conventional photolithography, the wafers were annealed under dry nitrogen at $800{ }^{\circ} \mathrm{C}$ for $60 \mathrm{~min}$. After exposure and development of the mask patterns (hexagonal arrangement of $100 \mu \mathrm{m}$ circular features at $200 \mu \mathrm{m}$ periodicity) into positive photoresist (Fig. 6.3a), transfer of this pattern into $\mathrm{LPCVD}-\mathrm{SiO}_{2}$ was done by etching in $1 \% \mathrm{HF}$ for $1 \mathrm{~min}$ (Fig. 6.3b). After a subsequent photoresist strip in acetone/ isopropanol, the $\mathrm{LPCVD}-\mathrm{SiO}_{2}$ pattern was transferred into $\mathrm{Si}_{3} \mathrm{~N}_{4}$ by etching in $85 \% \mathrm{H}_{3} \mathrm{PO}_{4}$ at $180{ }^{\circ} \mathrm{C}$ for $5 \mathrm{~min}$. The resulting $\mathrm{Si}_{3} \mathrm{~N}_{4}$ oxidation mask for LOCOS is shown in Fig. 6.3c after stripping the $\mathrm{LPCVD}-\mathrm{SiO}_{2}$ in $1 \% \mathrm{HF}$ for $2 \mathrm{~min}$. The low intrinsic etch rates of the underlying layers, i.e., $\mathrm{Si}_{3} \mathrm{~N}_{4}$ in $1 \% \mathrm{HF}$ and silicon in $85 \% \mathrm{H}_{3} \mathrm{PO}_{4}$ (Table 6.1) during the patterning of respectively $\mathrm{LPCVD}-\mathrm{SiO}_{2}$ and $\mathrm{Si}_{3} \mathrm{~N}_{4}$, allow substantial over-etching without the problem of undercutting and/or damage of the underlying layer. Feature dimension analysis of nanometer-scale features in Chapter 7 illustrates a total increase of about $20 \mathrm{~nm}$ in feature dimension (= $=8.6 \%$ of the initial feature dimensions) during the successive transfer of the resist pattern into the underlying two transfer layers. Finally, the substrates were put in a dry 
oxidation furnace at $950{ }^{\circ} \mathrm{C}$ to perform the actual pattern inversion step by LOCOS using the ultrathin $\mathrm{Si}_{3} \mathrm{~N}_{4}$ patterns as oxidation masks. Under these conditions, the oxidation rate of silicon into thermal $\mathrm{SiO}_{2}$ was about $1.2 \mathrm{~nm} \cdot \mathrm{min}^{-1}$, resulting in $14 \mathrm{~nm}$ of thermal $\mathrm{SiO}_{2}$ after 12 min oxidation. ${ }^{[18]}$ The resulting thermal $\mathrm{SiO}_{2}$ etch mask after stripping the $\mathrm{Si}_{3} \mathrm{~N}_{4}$ in $85 \% \mathrm{H}_{3} \mathrm{PO}_{4}$ at $180{ }^{\circ} \mathrm{C}$ is shown in Fig. 6.3d.
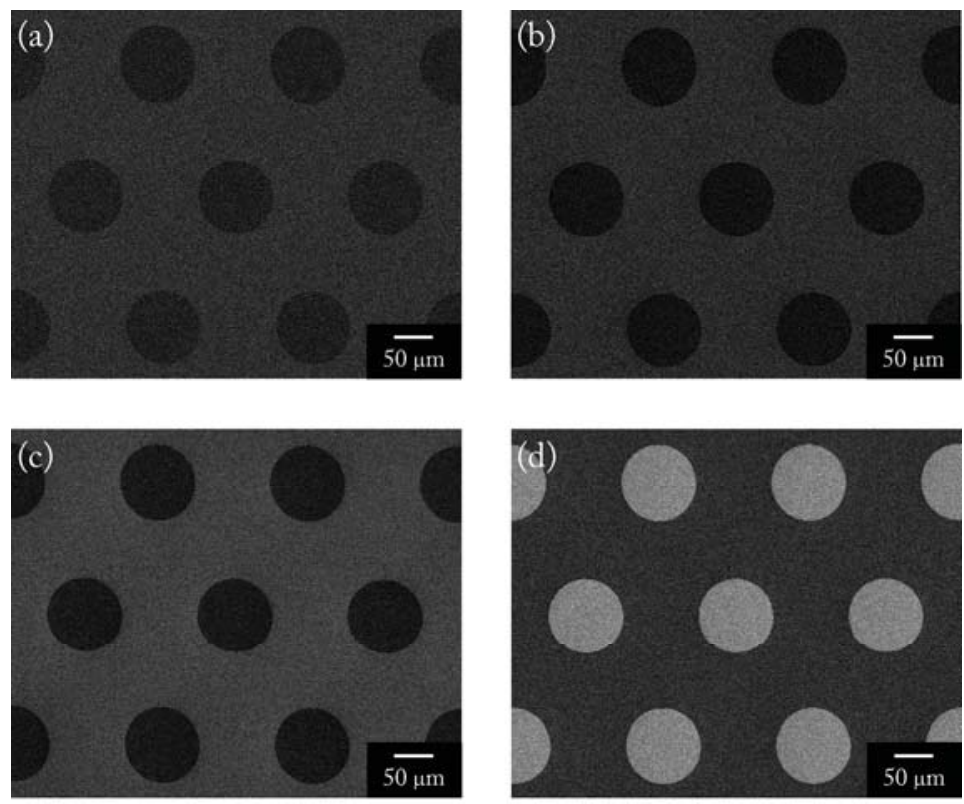

Figure 6.3 SEM images of $100 \mu \mathrm{m}$ circular features in a hexagonal pattern at a periodicity of $200 \mu \mathrm{m}$ : circular holes in photoresist Olin 907 (a), in $\mathrm{LPCVD}-\mathrm{SiO}_{2}$ (b), and in $\mathrm{Si}_{3} \mathrm{~N}_{4}$ (c) and inverse circular features in thermal $\mathrm{SiO}_{2}(\mathrm{~d})$.

The entire inversion procedure was found to occur with very high accuracy due to the lateral encroachment of thermal $\mathrm{SiO}_{2}$ under the oxidation mask (deviation in lateral feature dimensions of $<4 \mathrm{~nm}$, see Chapter 7). The time to strip the $\mathrm{Si}_{3} \mathrm{~N}_{4}$ oxidation mask from the substrate after LOCOS took longer than the theoretical etch time (Table 6.1) due to partial oxidation of $\mathrm{Si}_{3} \mathrm{~N}_{4}$ during the oxidation run in the furnace. An additional $2 \mathrm{~min}$ strip (at a total of $7 \mathrm{~min}$ ) was necessary to overcome the initial etch resistance to $85 \% \mathrm{H}_{3} \mathrm{PO}_{4}$ at $180{ }^{\circ} \mathrm{C}$, using the hydrophobicity of the silicon as a visual etch stop during rinsing of the substrates with water. As a result, the final thermal $\mathrm{SiO}_{2}$ etch mask was about $12 \mathrm{~nm}$ thick. Complete removal of the $\mathrm{Si}_{3} \mathrm{~N}_{4}$ oxidation mask was carried out just before silicon etching to prevent re-oxidation of the silicon to native oxide. 


\subsubsection{Profile control by cryogenic etching}

The requirements of anisotropic etching on the sub-500 $\mathrm{nm}$ scale using ultrathin $(12 \mathrm{~nm})$ thermal $\mathrm{SiO}_{2}$ etch masks are stringent. These dimensions necessitate the development of a suitable anisotropic etch recipe that combines a low silicon etch rate with a silicon to thermal $\mathrm{SiO}_{2}$ etch selectivity of at least 30 for achieving accurate silicon etching. For several reasons, the Oxford Plasmalab 100 plus system was chosen to cryogenically etch the final patterns into silicon. This system includes helium backside cooling by liquid nitrogen in combination with a mechanical clamping tool that allows efficient control of the temperature over the entire 4-inch wafer. Additionally, the dual-power plasma source of this system enables independent control of the plasma density (set by the inductive coupled plasma (ICP) power) and bias voltage (set by the capacitive coupled plasma (CCP) power) that affect, respectively, the chemical (by radicals) and physical (by ions) components of the silicon etching. The following describes the procedure to formulate a suitable recipe by complying with the guidelines for profile control at cryogenic temperatures using $\mathrm{SF}_{6} / \mathrm{O}_{2}$ plasmas. ${ }^{[19]}$ At first, some of the input parameters were intentionally set for specific reasons: (i) ICP power of $550 \mathrm{~W}$ (= minimum ICP power of the etch system) in order to generate a low plasma density, resulting in low silicon etch rates, (ii) process pressure of $10 \mathrm{~m}$ Torr in order to achieve directional ion bombardment during etching, and (iii) electrode temperature of $-120^{\circ} \mathrm{C}$ in order to obtain high etch selectivity. After setting these input parameters, the flow of $\mathrm{SF}_{6}$ and $\mathrm{O}_{2}$ and the $\mathrm{CCP}$ power are the only active input parameters necessary to tune the profile. The second stage was to determine the (spontaneous) silicon etch rate as a function of the $\mathrm{SF}_{6}$ flow on bare 4-inch silicon wafers.

Fig. 6.4a illustrates that the silicon etch rate in this particular etch regime is proportional to the $\mathrm{SF}_{6}$ flow at a rate dependence of $20 \mathrm{~nm} \cdot \mathrm{min}^{-1} \cdot \mathrm{sccm}^{-1}$. One drawback and limiting factor of this system is the quartz dome construction inside the reactor. Erosion of this type of dome during etching is known to cause oxygen emission, thereby affecting the etching properties of the plasma. ${ }^{[20]}$ As a result, the third stage was to quantify this effect by determining the silicon etch rate as a function of the CCP power. 
(a)

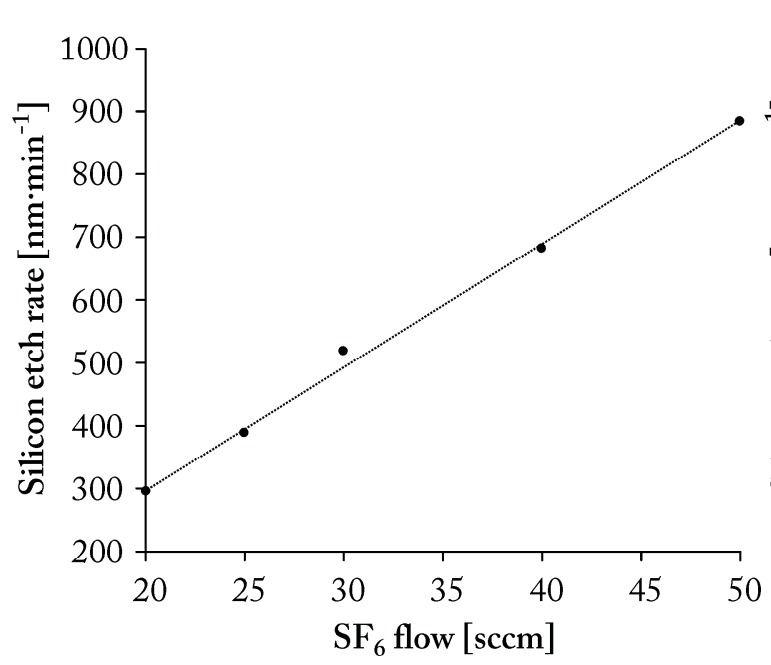

(b)

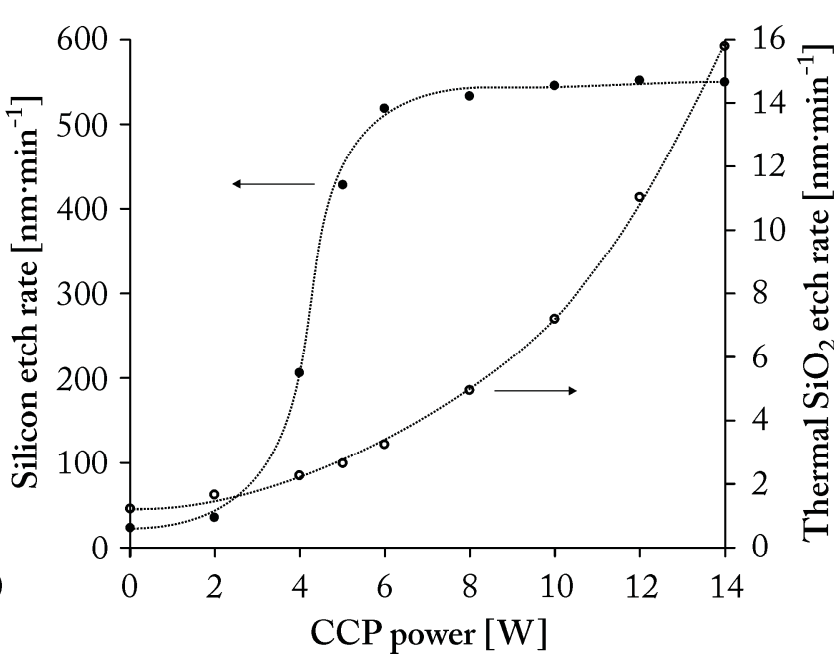

Figure 6.4 Plot of the etch rate dependence on different parameter settings using the Oxford Plasmalab 100 plus system. General parameter settings: $10 \mathrm{mTorr}, 550 \mathrm{~W} \mathrm{ICP}$ power, $-120{ }^{\circ} \mathrm{C}$ (no $\mathrm{O}_{2}$ addition). (a) Silicon etch rate as a function of $\mathrm{SF}_{6}$ flow at $6 \mathrm{~W} \mathrm{CCP}$ power. (b) Silicon and thermal $\mathrm{SiO}_{2}$ etch rates as a function of $\mathrm{CCP}$ power at $30 \mathrm{sccm} \mathrm{SF}_{6}$. Etch rate determination was done on 4-inch wafers by weight measurement in case of silicon and by ellipsometry in case of thermal $\mathrm{SiO}_{2}$. The resulting etch rates apply to a $100 \%$ load.

Fig. $6.4 \mathrm{~b}$ illustrates that the passivation by oxygen from the dome was only effective at low $\mathrm{CCP}$ powers $(\mathrm{CCP}$ power $<6 \mathrm{~W})$. At higher $\mathrm{CCP}$ powers $(>6 \mathrm{~W})$, the silicon etch rate was independent of the $\mathrm{CCP}$ power due to the fact that the corresponding higher bias voltages overpower the threshold energy for physical sputtering of the oxygen-containing passivation layer. In contrast, the etch rate of the thermal $\mathrm{SiO}_{2}$ was dependent on the CCP power over the entire regime, as shown in Fig. 6.4b, as erosion of this masking material is physical by nature. On the basis of these findings, the profile test experiments were carried out on a 4 -inch wafer scale using $12 \mathrm{~nm}$ thick patterns of thermal $\mathrm{SiO}_{2}$ as etch masks (for the fabrication, see Fig. 6.5 (left)).

Fig. 6.5 (profile I) illustrates a positive profile under the starting etch conditions (30 sccm $\mathrm{SF}_{6}$, no $\mathrm{O}_{2}$ addition, $6 \mathrm{~W}$ CCP power), indicating that the oxygen content in the plasma was already sufficient to cause strong sidewall passivation. Following the guidelines for profile control, ${ }^{[19,20]}$ tuning of this profile into a more directional profile (Fig. 6.5, profile II) is possible by either increasing the $\mathrm{SF}_{6}$ flow (Fig. 6.5, step 1b) or the CCP power (Fig. 6.5, step 1a): ${ }^{[21]}$ increasing the $\mathrm{SF}_{6}$ flow (at constant pressure) reduces the strength of the passivation layer, whereas increasing the $\mathrm{CCP}$ power intensifies the sputtering of this passivation layer. 


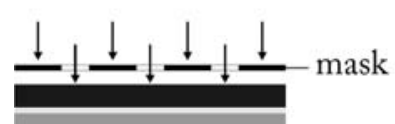

exposure and development
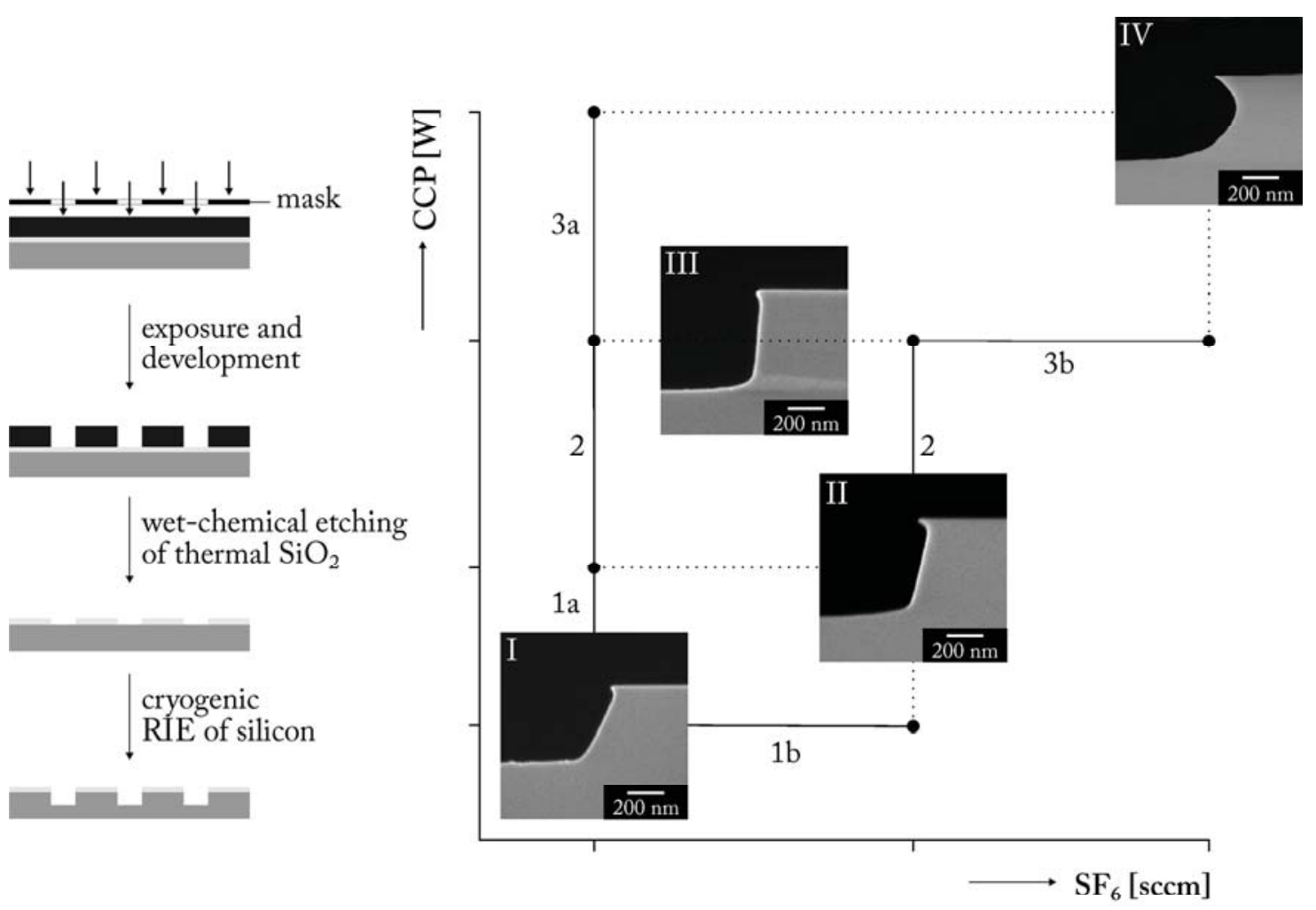

$<100>$ silicon thermal $\mathrm{SiO}_{2}(12 \mathrm{~nm})$

Olin 907/12 (1.2 $\mu \mathrm{m})$

Figure 6.5 Schematic representation of the entire profile test experiment (left) and the resulting diagram of the procedure for ultimate profile control including high-resolution SEM cross-sectional profiles (right). The diagram illustrates different possible etch profiles: ${ }^{[22]}$ positive profile (I), directional profile (II and III), and semi-isotropic profile (IV).

However, Fig. 6.6 illustrates that the resulting etch selectivity of silicon over thermal $\mathrm{SiO}_{2}$ is different in these two cases, due to the difference in etch rates of silicon and thermal $\mathrm{SiO}_{2}$ (Fig. 6.4). Additionally, Fig. 6.6 illustrates that, at the set conditions in stage $1\left(550 \mathrm{~W}\right.$ ICP power, $\left.10 \mathrm{mTorr},-120^{\circ} \mathrm{C}\right)$, there is a large process latitude in the cryogenic etching of silicon in terms of the selectivity of silicon over thermal $\mathrm{SiO}_{2}$, i.e., in the present range of $\mathrm{CCP}$ power $(6-12 \mathrm{~W})$ and $\mathrm{SF}_{6}$ flow $(30-50 \mathrm{sccm})$ the selectivity is sufficient to formulate an anisotropic etch recipe with a suitable selectivity (>30). An increase of any of the two parameters out of this range was found to result in semi-isotropic profiles (Fig. 6.5, step 3a/b and profile IV). 


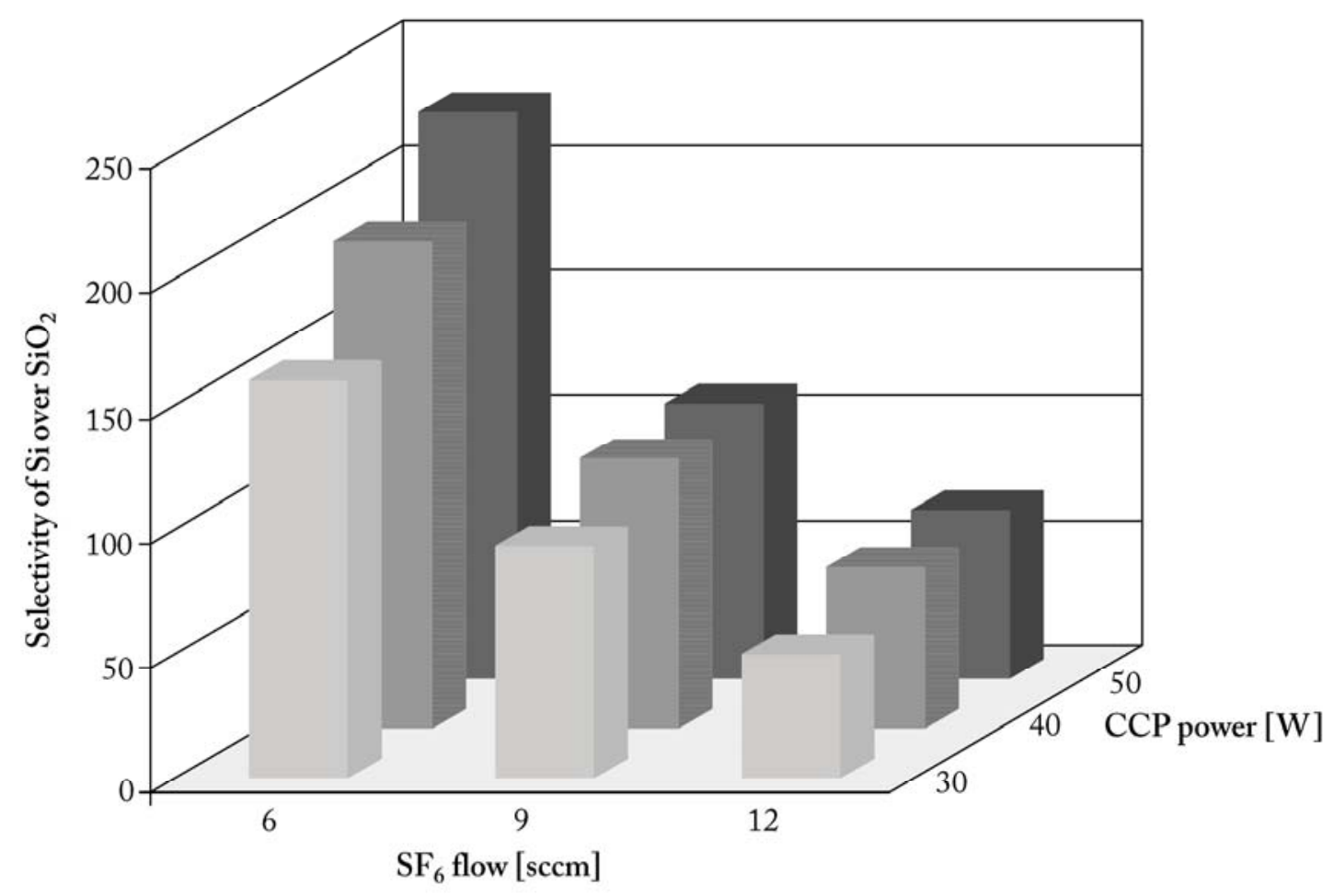

Figure 6.6 Etch selectivity of silicon over thermal $\mathrm{SiO}_{2}$ as a function of the $\mathrm{SF}_{6}$ flow $(30-50 \mathrm{sccm})$ and $\mathrm{CCP}$ power (6-12 W). Selectivities in this etch regime vary from 50 (at $30 \mathrm{sccm} \mathrm{SF}_{6}$ and $12 \mathrm{~W} \mathrm{CCP}$ power) up to 225 (at $50 \mathrm{sccm} \mathrm{SF}_{6}$ and $6 \mathrm{~W} \mathrm{CCP}$ ).

Fig. 6.5 and 6.6 indicate that increasing the $\mathrm{SF}_{6}$ flow or the $\mathrm{CCP}$ power will result in two different anisotropic etch recipes. The former action results in a high etch selectivity at the expense of high silicon etch rates, whereas the latter action results in a low silicon etch rate at the expense of low etch selectivities. Upon considering that the target is to formulate a recipe with a low silicon etch rate, preference was given to increase the CCP power first for substantial tuning (Fig. 6.5, step 1a and 2: + $6 \mathrm{~W}$ ) of the profile prior to increasing the $\mathrm{SF}_{6}$ flow $(+4 \mathrm{sccm})$ for final fine-tuning of the profile. Conditions necessary for close-to-anisotropic etching (Fig. 6.5, profile III) were found using this systematic procedure with the following conditions: $10 \mathrm{mTorr}$, $550 \mathrm{~W}$ ICP power, $12 \mathrm{~W} \mathrm{CCP}$ power, $34 \mathrm{sccm} \mathrm{SF} 6,-120{ }^{\circ} \mathrm{C}$ (quartz dome). Using these settings, the silicon etch rate is about $600 \mathrm{~nm} \cdot \mathrm{min}^{-1}$ and the selectivity of silicon over thermal $\mathrm{SiO}_{2}$ is about 50 . 


\subsection{Conclusions}

The results in this chapter illustrate that the present LOCOS pattern inversion procedure to convert resist patterns into inverse patterns of thermal $\mathrm{SiO}_{2}$ is very accurate in terms of pattern transfer accuracy (total deviation in lateral dimension of $<4 \mathrm{~nm}^{[23]}$ ) and feature definition owing to the use of ultrathin functional layers, wetchemical etching and process engineering of the etch rate and selectivity. This lithographic procedure offers advantages over the conventional multilayer resist systems for metal lift-off in terms of transfer accuracy and process complexity (no need for undercut profile, no successive plasma etch steps) in case the resulting pattern serves as an etch mask for the anisotropic etching of silicon.

One technological improvement in the present inversion procedure includes the use of hydrogen silsesquioxane (HSQ) instead of the combination of photoresist/ LPCVD-SiO ${ }_{2}$. HSQ (structural formula: $\mathrm{H}_{2}(\mathrm{SiO})_{3}$ ) is commercially available in solution as FOX $^{\circledast}$ flowable oxide (Dow Corning ${ }^{[24]}$ ) and is of interest for four reasons: (i) potential application in room-temperature NIL (RT-NIL), ${ }^{[25]}$ pattern alignment and step-and-repeat patterning, (ii) high etch resistance, (iii) tunable properties by thermal curing, ${ }^{[26]}$ and (iv) effective reduction of the number of transfer layers.

Finally, the cryogenic RIE of silicon in the sub-500 nm scale using the ultrathin thermal $\mathrm{SiO}_{2}$ etch masks was successful by application of a systematic etch procedure to formulate a suitable anisotropic etch recipe (silicon etch rate of $600 \mathrm{~nm} \cdot \mathrm{min}^{-1}$ at etch selectivity with respect to thermal $\mathrm{SiO}_{2}$ of about 50 ).

\subsection{Experimental}

Chemicals and materials: All chemicals and materials were purchased from commercial resources: 4-inch OSP $<100>$ p-type silicon wafers (Okmatic), Olin 907/12 (Fujifilm Arch), hexamethyldisilazane (HMDS) (100\% Selectipur: Merck), OPD 4262 resist developer (Arch Chemicals), $\mathrm{HNO}_{3}$ (100\% Selectipur: Merck, and 69\% VLSI: Merck), HF (1\% VLSI: Merck), $\mathrm{H}_{3} \mathrm{PO}_{4}$ (85\% VLSI, Merck), acetone and isopropanol (Merck, VLSI), tetraethylorthosilicate (TEOS) (Schumacher Company), dichlorosilane $\left(\mathrm{SiH}_{2} \mathrm{Cl}_{2}\right)$ (PraxAir, 2.0) and $\mathrm{NH}_{3}$ (PraxAir, 5.0). All experiments were carried out in cleanroom environment. 
Substrate preparation: Prior to processing the silicon wafers, the native oxide was removed in $1 \% \mathrm{HF}$ for $60 \mathrm{~s}$. Afterwards, a very thin layer of stoichiometric silicon nitride $\left(\mathrm{Si}_{3} \mathrm{~N}_{4}, 14 \mathrm{~nm}\right)$ was deposited by low-pressure chemical vapor deposition (LPCVD, Tempress LPCVD furnace: $70 \mathrm{sccm} \mathrm{SiH}_{2} \mathrm{Cl}_{2}, 18 \mathrm{sccm} \mathrm{NH}_{3}, 850{ }^{\circ} \mathrm{C}$, 200 mTorr, $150 \mathrm{~s}$ deposition time) with minimal delay. On top of this layer, a very thin layer of LPCVD-SiO ${ }_{2}(12 \mathrm{~nm})$ was deposited by LPCVD (Tempress LPCVD furnace: $50 \mathrm{sccm}$ TEOS, $700{ }^{\circ} \mathrm{C}, 400 \mathrm{mTorr}$, bubbler at $40{ }^{\circ} \mathrm{C}, 90 \mathrm{~s}$ deposition time). After the two LPCVD runs, the wafers were annealed under dry nitrogen at $800^{\circ} \mathrm{C}$ for $60 \mathrm{~min}$ in a furnace (Tempress, dry oxidation furnace) with minimal delay.

Fabrication of resist patterns: Resist patterns were fabricated on the 4-inch wafer scale by conventional optical lithography (ElectroVisions Group mask aligner (EVG), model EV620). The wafers were cleaned by a standard cleaning procedure (10 $\mathrm{min}$ in $70 \% \mathrm{HNO}_{3}, 10 \mathrm{~min}$ in $100 \% \mathrm{HNO}_{3}$ at $95^{\circ} \mathrm{C}$ ). A dehydration bake on the hotplate $\left(120{ }^{\circ} \mathrm{C}, 5 \mathrm{~min}\right.$ ) was applied on the wafers before priming with HMDS. Spin-coating of the positive photoresist Olin 907/12 was done at $4000 \mathrm{rpm}$ for $20 \mathrm{~s}$. A softbake $\left(95^{\circ} \mathrm{C}, 60 \mathrm{~s}\right)$ was preceding the optical exposure of the resist $\left(3 \mathrm{~s}, 12 \mathrm{~mW} \cdot \mathrm{cm}^{-2}\right.$, hard contact with 0.7 bar contact pressure) through a mask. After a post-exposure bake at $120{ }^{\circ} \mathrm{C}$ for $60 \mathrm{~s}$, the resist was developed by mild agitation in OPD 4262 resist developer for $50 \mathrm{~s}$.

Local oxidation of silicon (LOCOS): LOCOS requires the transfer of the photoresist pattern into two different layers: first into the $\mathrm{LPCVD}-\mathrm{SiO}_{2}$ layer by etching in $1 \% \mathrm{HF}$ for $45 \mathrm{~s}$ and, after removal of the PMMA from the substrates in acetone/ isopropanol, into $\mathrm{Si}_{3} \mathrm{~N}_{4}$ by etching in hot $\mathrm{H}_{3} \mathrm{PO}_{4}$ of $180{ }^{\circ} \mathrm{C}$ for $5 \mathrm{~min}$. After removal of the $\mathrm{LPCVD}-\mathrm{SiO}_{2}$ mask in a $1 \% \mathrm{HF}$ solution for $2 \mathrm{~min}$, the silicon was locally oxidized in a dry oxidation furnace (Tempress, dry oxidation furnace, atmospheric pressure, $4000 \mathrm{sccm} \mathrm{O}_{2}, 950{ }^{\circ} \mathrm{C}, 12 \mathrm{~min}$ ) using the $\mathrm{Si}_{3} \mathrm{~N}_{4}$ as the oxidation mask. Removal of the $\mathrm{Si}_{3} \mathrm{~N}_{4}$ mask in $\mathrm{H}_{3} \mathrm{PO}_{4}$ at $180{ }^{\circ} \mathrm{C}$ for 7 min was performed just before the cryogenic etching of the silicon to prevent the formation of native silicon oxide.

Cryogenic reactive ion etching of silicon: Silicon etching in the sub-500 $\mathrm{nm}$ scale was carried out on the Plasmalab 100 plus system from Oxford Instruments. After loading the wafer into the etch reactor, a holding time for etching was set to $2 \mathrm{~min}$ for the system to equilibrate the temperature of the substrate. For the formulation of an 
anisotropic etch recipe, the resulting cross-sectional profiles were examined by highresolution SEM after etching and manual cleavage of the silicon wafer. After setting the ICP power $(550 \mathrm{~W})$, the pressure $(10 \mathrm{mTorr})$ and the temperature $\left(-120^{\circ} \mathrm{C}\right)$, adjustment of the $\mathrm{SF}_{6}$ flow and $\mathrm{CCP}$ power was done systematically to reach the anisotropic profile. Parameter settings for the anisotropic etching of silicon are found at $10 \mathrm{~m}$ Torr, $550 \mathrm{~W}$ ICP power, $12 \mathrm{~W} \mathrm{CCP}$ power, $34 \mathrm{sccm} \mathrm{SF} 6,-120{ }^{\circ} \mathrm{C}$. At these settings, the silicon etch rate is about $600 \mathrm{~nm} \cdot \mathrm{min}^{-1}$ and the selectivity of silicon over thermal $\mathrm{SiO}_{2}$ is about 50 .

Characterization: Layer thicknesses on silicon were determined with a Plasmos SD 2002 ellipsometer operating at a wavelength of $632.8 \mathrm{~nm}$. The weight measurements were carried out on a Sartorius weighing scale with an accuracy of $0.1 \mathrm{mg}$ (conversion factor: $1 \mathrm{mg}=55 \mathrm{~nm}$ ). Low-resolution SEM imaging was carried out with a JEOL JSM-5610 SEM operating in secondary electron detection. High-resolution SEM imaging was carried out with a LEO Gemini 1550 FEG-SEM operating in the INLENS mode for secondary electron detection.

\subsection{References and notes}

[1] E. Reichmanis, L. F. Thompson, Chem. Rev. 1989, 89, 1273.

[2] W. T. S. Huck, Int. J. Nanotech. 2003, 1-2, 119.

[3] S. Y. Chou, P. R. Krauss, P. J. Renstrom, Science 1996, 272, 85.

[4] S. Y. Chou, P. R. Krauss, W. Zhang, L. Guo, L. Zhuang, J. Vac. Sci. Technol. B 1997, 15, 2897.

[5] (a) B. Faircloth, H. Rohrs, R. Tiberio, R. Ruoff, R. R. Krchnavek, J. Vac. Sci. Technol. B 2000, 18, 1866. (b) P. Carlberg, M. Graczyk, E.-L. Sarwe, I. Maximov, M. Beck, L. Montelius, Microelectron. Eng. 2003, 67-68, 203. (c) K. Nakamatsu, K. Watanabe, K. Tone, T. Katase, W. Hattori, Y. Ochiai, T. Matsuo, M. Sasago, H. Namatsu, M. Komuro, S. Matsui, Jpn. J. Appl. Phys. 2004, 43, 4050.

[6] (a) A. Lebib, Y. Chen, F. Carcenac, E. Cambril, L. Manin, L. Couraud, H. Launois, Microelectron. Eng. 2000, 53, 175. (b) S. H. Kim, H. Hiroshima, S. Inoue, Y. Kurashimma, M. Komuro, J. Vac. Sci. Technol. B 2003, 21, 3144. (c) J. Shi, C. Peroz, D. Peyrade, J. Salari, M. Belotti, W. H. Huang, Y. Chen, Microelectron. Eng. 2006, 83, 1664.

[7] J. A. Appels, E. Kooi, M. M. Paffen, J. J. H. Schatorje, W. H. C. G. Verkuylen, Philips Res. Reports 1970, 25, 118. 
[8] The primary purpose of isolation technology in semiconductor device fabrication is to electrically isolate devices on the wafer by the use of field oxides.

[9] J. W. Lutze, A. H. Perera, J. P. Krusius, J. Electrochem. Soc. 1990, 137, 1867.

[10] S. Deleonibus, Solid-State Electron. 1997, 41, 1027.

[11] Etch rate determination was done by constructing etch plots containing five data points at different etch times. Each of the five data points was taken as the average of a 25 -point ellipsometric measurement of the layer thickness before and after etching of $5 \times 5 \mathrm{~cm}^{2}$ substrates. Refractive indices of the different materials: $1.42\left(\mathrm{LPCVD}-\mathrm{SiO}_{2}\right), 2.00\left(\mathrm{Si}_{3} \mathrm{~N}_{4}\right)$, and 1.465 (thermal- $\mathrm{SiO}_{2}$ ).

[12] W. van Gelder, V. E. Hauser, J. Electrochem. Soc. 1967, 114, 869.

[13] (a) K. R. Williams, R. S. Muller, J. Microelectromech. Sys. 1996, 5, 256. (b) K. R. Williams, K. Gupta, M. Wasilik, J. Microelectromech. Sys. 2003, 12, 761.

[14] (a) S. Rojas, A. Modelli, W. S. Wu, A. Borghesi, B. Pivac, J. Vac. Sci. Technol. B 1990, 8, 1177. (b) C. Orfescu, C. Pavelescu, M. Badila, J. Mater. Sci. 1990, 25, 1366.

[15] I. Fränz, W. Langheinrich, Solid-State Electron. 1971, 14, 499.

[16] L. V. Chramova, T. P. Smirnova, B. M. Ayupov, V. I. Belyi, Thin Solid Films, 1981, 78, 303.

[17] The LPCVD furnace limits the ultimate layer thickness of the $\mathrm{Si}_{3} \mathrm{~N}_{4}$ due to the slow response of the mass flow controllers to set the mass flow to the correct settings. Stabilization of the mass flows during the first minute of deposition results in a starting $\mathrm{Si}_{3} \mathrm{~N}_{4}$ thickness of 7-8 $\mathrm{nm}$. Additionally, this system starts to deliver good $\mathrm{Si}_{3} \mathrm{~N}_{4}$ layers of constant quality from a thickness of $12 \mathrm{~nm}$.

[18] The specific volume of $\mathrm{SiO}_{2}$ is about 2.2 times that of silicon, so $45 \%$ of the resulting LOCOS structures reside below the surface of the substrate.

[19] (a) H. Jansen, M. J. de Boer, R. Legtenberg, M. Elwenspoek, J. Micromech. Microeng. 1995, 5, 115. (b) H. Jansen, M. J. de Boer, H. Wensink, B. Kloeck, M. Elwenspoek, Microelectron. J. 2001, 32, 769 .

[20] M. J. de Boer, J. G. E. Gardeniers, H. V. Jansen, E. Smulders, M.-J. Gilde, G. Roelofs, J. N. Sasserath, M. Elwenspoek, J. Microelectromech. Sys. 2002, 11, 385.

[21] Profile control is to find the delicate balance between etching and sidewall passivation. Of all input parameters, the $\mathrm{O}_{2}$ content of the plasma is the most important for profile control in $\mathrm{SF}_{6} / \mathrm{O}_{2}$ plasmas at cryogenic temperatures as it determines the quality of the passivation layer (in terms of thickness and composition). In the case of a positive profile, any action that causes a decrease in the quality of the passivation layer $\left(\mathrm{SF}_{6}\right.$ flow and temperature) or an increase of the ion energy flux (CCP in combination with $\mathrm{ICP}$ ) towards the electrode results in a more directional and anisotropic profile. 
[22] The exact cause of the undercut in the top part of the profiles is unknown, but could find its origin during start up of the plasma by the apparatus.

[23] This specific transfer accuracy is shown in Chapter 7 by feature dimension analysis of nanometer-scale features.

[24] www.dowcorning.com

[25] Y. Igaku, S. Matsui, H. Ishigaki, J. Fujita, M. Ishida, Y. Ochiai, H. Namatsu, M. Komuro, H. Hiroshima, Jpn. J. Appl. Phys. 2002, 41, 4198.

[26] (a) H.-C. Liou, J. Pretzer, Thin Solid Films 1998, 335, 186. (b) Y. K. Siew, G. Sarkar, X. Hu, J. Hui, A. See, C. T. Chua, J. Electrochem. Soc. 2000, 147, 335. 


\section{Fabrication, replication and characterization of 2D photonic crystal waveguides"}

This chapter describes a novel inverse imprinting procedure for the fabrication and replication of two-dimensional photonic crystal waveguides with a transfer accuracy and feature definition that is comparable to state-of-the-art nanofabrication techniques. Local examination using photon scanning tunneling microscopy (PSTM) demonstrates that the resulting nanophotonic structures have excellent guiding properties at wavelengths in the telecommunications range, indicating a bigh quality of the local structure and the overall periodicity.

\subsection{Introduction}

Photonic crystals $(\mathrm{PhCs})^{[1,2]}$ have been a fascinating and active field of research in the past decade owing to the ability of these artificial nanostructures to influence the generation and propagation of light. ${ }^{[3,4]}$ In particular, due to the periodic dielectric

"Part of this chapter has been published in: C. M. Bruinink, M. Burresi, M. J. de Boer, F. B. Segerink, H. V. Jansen, E. Berenschot, D. N. Reinhoudt, J. Huskens, L. Kuipers, Nano Lett. 2008, 8, 2872-2877 (Copyright $@ 2008$ American Chemical Society). 
structure, $\mathrm{PhCs}$ may prohibit the propagation of photons of a certain range of frequencies (known as the photonic band gap). The flexibility to tune the optical properties by changing the local geometry in PCs results in novel types of filters, couplers, lasers, ${ }^{[5]}$ waveguides, ${ }^{[6]}$ etc. For example, by incorporating structural line defects within a (perfect) two-dimensional $\mathrm{PhC}$ in thin dielectric slabs one can fabricate low-loss two-dimensional photonic crystal waveguides (2D PhCWGs). The strong confinement of light in the line defect of 2D PhCWGs in principle surpasses the guiding properties of conventional waveguides in terms of propagation losses owing to the in-plane band gap confinement. The fabrication of these 2D PhCWGs on oxide claddings of commercially available high-quality silicon-on-insulator (SOI) wafers has become the most dominant in scientific research as it allows the direct application of the mature tools of silicon nanofabrication technology, e.g., direct e-beam lithography (EBL) ${ }^{[7]}$ and deep-UV (DUV) lithography, ${ }^{[8]}$ for high-resolution pattern generation in combination with any deposition and/or dry etch tool to transfer the final devices into SOI. ${ }^{[9]}$

Up to now, most research has been focusing on the theoretical and experimental investigation of the guiding properties of $2 \mathrm{D}$ PhCWGs in terms of specific design rules of different elements of $2 \mathrm{D} \mathrm{PhCWG}$, propagation losses, and light coupling efficiencies. Less attention has been given to the low-cost fabrication of 2D PhCWGs so far. Generally, nanophotonic devices pose very stringent requirements with regard to the quality of the nanofabrication technique. Not only does the definition of the local features, typically nanoholes in a background of high-index material, need to be perfect, but also the period of the crystal lattice has to be constant throughout the entire device. While local imperfections will lead to scattering losses, long-range imperfections, such as a non-constant period, will lead to undesirable variations in the propagation constant and potentially in reflections and out-of-plane losses. From the set of emerging nanofabrication techniques, nanoimprint lithography (NIL) ${ }^{[10]}$ has the highest potential for becoming the next-generation high-resolution lithography because of its high transfer accuracy, cost effectiveness and high throughput even for structures that require sub-10 $\mathrm{nm}$ resolution. The research and development of NIL as a tool in the fabrication process of $2 \mathrm{D} \mathrm{PhCWGs} \mathrm{is} \mathrm{only} \mathrm{very} \mathrm{recent.} \mathrm{After} \mathrm{the} \mathrm{first}$ successful demonstration of low-cost replication of PhCWGs in polymers by thermal 
NIL ${ }^{[11]}$ in 2005, only two research groups have put effort into the replication of 2D PhCWGs on SOI by exploiting NIL. ${ }^{[12,13]}$

The research in this chapter elaborates on the LOCOS inversion procedure and design rules set in Chapter 6, resulting in the highest pattern transfer accuracy (in terms of feature dimension loss and feature definition) to date in the replication of 2D $\mathrm{PhCWG}$ into SOI. The novelty with respect to the current multilayer resist systems ${ }^{[14,15]}$ lies in the fact that this procedure implements, for the first time, local oxidation of silicon (LOCOS), ${ }^{[16]}$ a microfabrication isolation technique for verylarge-scale integration (VLSI), in the inversion of NIL resist patterns into thermal silicon oxide $\left(\mathrm{SiO}_{2}\right)$. As the pattern transfer accuracy was the most important output factor in the entire process evaluation, high-resolution scanning electron microscopy (SEM) imaging of the resulting pattern was carried out after every process step in the replication procedure. Additionally, photon scanning tunneling microscopy (PSTM) was carried out in order to study the $2 \mathrm{D}$ PhCWG in more detail by capturing the propagation of light inside the line defect of the $2 \mathrm{D}$ PhCWG to determine the actual propagation losses and band structure.

\subsection{Results and discussion}

\subsubsection{Structural design of the 2D PhCWG}

The structural design of the $2 \mathrm{D}$ PhCWG was taken from the excellent work of Notomi ${ }^{[17]}$ on single-mode transmission within the PBG of narrow line-defect $\mathrm{PhCWG}$ on SOI. Apart from setting up several guidelines for structural tuning of the waveguide modes of line-defect $\mathrm{PhCWG}$ by theoretical analysis of the dispersion curve of these modes within the $\mathrm{PBG}{ }^{[18]}$ superior single-mode transmission efficiencies for near-infrared light of transverse-electric (TE) polarization in the telecommunications range have been experimentally shown for two novel line-defect $\mathrm{PhCWG}$ structures on SOI. This was the result after structural optimization of the line defect of the 2D PhCWG in two different approaches: (i) by narrowing the width of the line defect ${ }^{[19]}$ and (ii) by addition of smaller holes inside the single line defect of the $2 \mathrm{D}$ PhCWG. ${ }^{[20]}$ Fig. $7.1 \mathrm{a}$ shows the schematic representation of the structural 
design of the 2D PhCWG used in this study, which is close to the narrow line-defect 2D PhCWG of Notomi. ${ }^{[19 a]}$

(a)

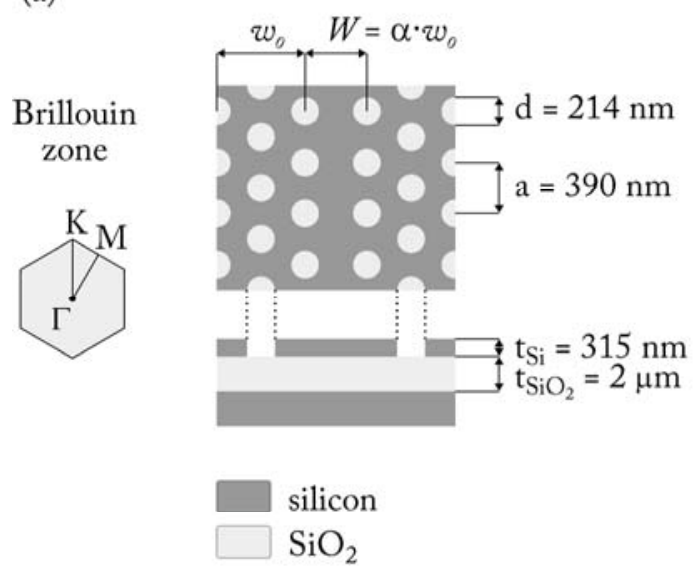

(b) 0.35

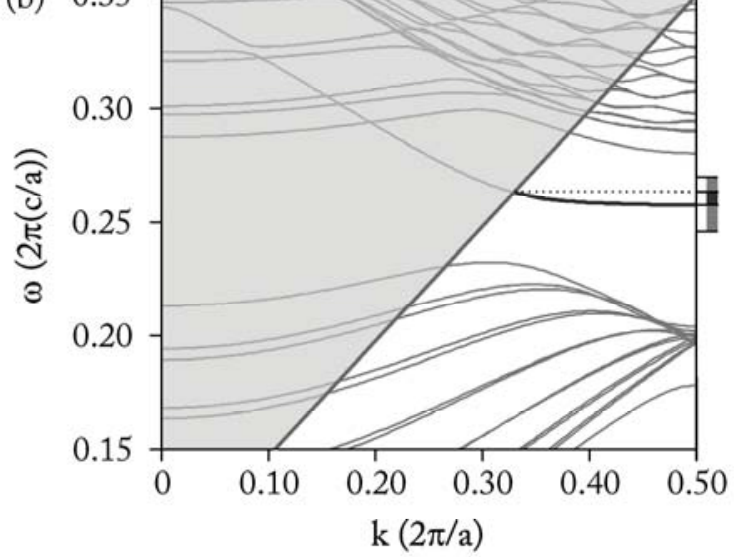

Frequency range laser $\quad-$ Light line of $\mathrm{SiO}_{2}$ Transmission band $\quad-$ Transmission mode Light cone

Figure 7.1 (a) Structural design of the 2D PhCWG. (b) Theoretical dispersion curve of this specific 2D PhCWG by calculation using a 2D plane-wave expansion method. The gray lines represent the TE-like waveguide modes, with the transmission mode in black. The light line of the $\mathrm{SiO}_{2}$ cladding layer indicates the border of the region inside the light cone of the cladding layer. The light gray areas correspond to the region outside the measurement range of the tunable laser.

The 2D PhCWGs consist of a triangular lattice of air holes of diameter $d$ at a lattice constant a perforating a $200 \mathrm{~nm}$ thick silicon slab that is on top of $2 \mu \mathrm{m}$ thick $\mathrm{SiO}_{2}$ cladding layer (Fig. 7.1a). Ridge waveguides ( $2 \mu \mathrm{m}$ wide) connect the $5 \times 22 \mu \mathrm{m}^{2}$ large 2D $\mathrm{PhCWG}$ to form the waveguide outline structure. At the fabrication stage of generating these waveguide outline structures, a lateral distance of $300 \mu \mathrm{m}$ in between the waveguides was set in the optical mask design for photolithography (Section 7.2.2). The line defect is along the $\Gamma-\mathrm{K}$ crystal direction of the $2 \mathrm{D} \mathrm{PhCWG}$ and has a line-width $W$ that is $70 \%(\alpha=0.7)$ of a normal single-line defect. This was done at the pre-fabrication stage of generating a stream file for FIB (Section 7.2.2) by removing a single line of air holes and shifting the structures in perpendicular direction with respect to the line defect.

A recalculation of the dispersion curve was necessary for the present $2 \mathrm{D} \mathrm{PhCWG}$ as the specifications of the SOI substrate were different from the $2 \mathrm{D}$ PhCWG of 
Notomi $\left(t_{\mathrm{Si}}=200 \mathrm{~nm}\right.$ and $\left.t_{\mathrm{Si}_{2}}=3 \mu \mathrm{m}\right)$ due to the availability of commercial SOI wafers at SOITEC. The geometric parameters of the final 2D PhCWG on SOI (see Section 7.2.3) were taken into account in this calculation. Fig. 7.1b illustrates the dispersion curve of the waveguiding modes using the $2 \mathrm{D}$ plane-wave expansion method of Johnson and Joannopoulos ${ }^{[21]}$ in combination with effective refractive-index calculations. ${ }^{[22]}$ Calculations predict a guiding mode for $\mathrm{TE}$ polarization (electrical field in the plane of the crystal) under the light line with a transmission band between $\omega=0.258$ and 0.264 . This transmission band is sufficient to propagate light of TE polarization inside the line defect of the $2 \mathrm{D} \mathrm{PhCWG}$ and lies within in the frequency range of the tunable laser of the PSTM setup (see Section 7.2.4).

\subsubsection{Fabrication of the nanoimprint mold}

The fabrication process starts with the fast prototyping of silicon molds containing 2D PhCWGs combining conventional optical lithography to define the micrometerscale ridge waveguides on larger areas and focused ion beam (FIB) milling to locally introduce the photonic crystal structure into the corresponding section within the waveguide outline structures (Scheme 7.1).

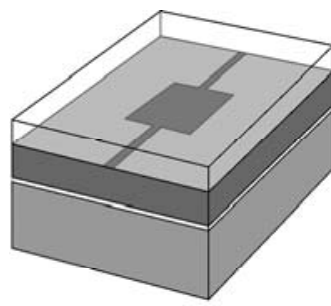

(a)

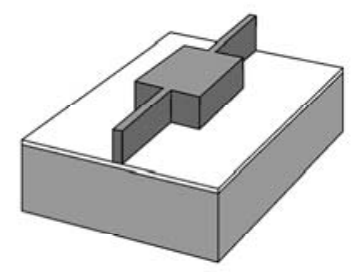

(b)

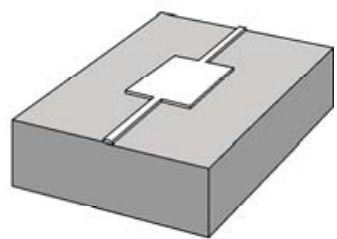

(c)

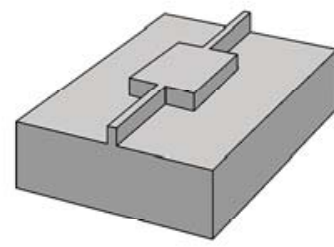

(d)

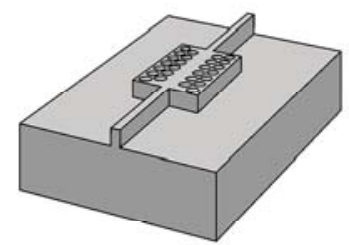

$<110>$ silicon

native $\mathrm{SiO}_{2}(\sim 2 \mathrm{~nm})$

photoresist (OLIN 907,1.2 $\mu \mathrm{m})$

Scheme 7.1 Schematic representation of the lithographic procedure for the fabrication of thermal NIL molds: (a) exposure of positive photosensitive resist through a mask containing the outline structures of the waveguides and subsequent resist development, (b) wet-chemical etching of the native $\mathrm{SiO}_{2}$, and removal of the photoresist patterns, (c) anisotropic wet-chemical etching of $<110>$ silicon exploiting the native $\mathrm{SiO}_{2}$ as the etch mask, and (d) local FIB milling of the photonic elements. 
After an introductory standard cleaning procedure and dehydration bake, HMDS and positive photosensitive resist Olin 907 were applied on the silicon wafer by spincoating. After a soft bake, the resulting $1.2 \mu \mathrm{m}$-thick resist layer was exposed by UV light through a mask containing the waveguide outline structures. Considering the anisotropic wet-chemical etching in this procedure, care was taken to align the direction of the ridge waveguides on the mask with the primary flat of the $<110>$ silicon wafer prior to exposure of the resist. After a post-exposure bake, the resist was developed in OPD 4262 resist developer (Scheme 7.1a), resulting in the waveguide outline patterns shown in Fig. 7.2a.
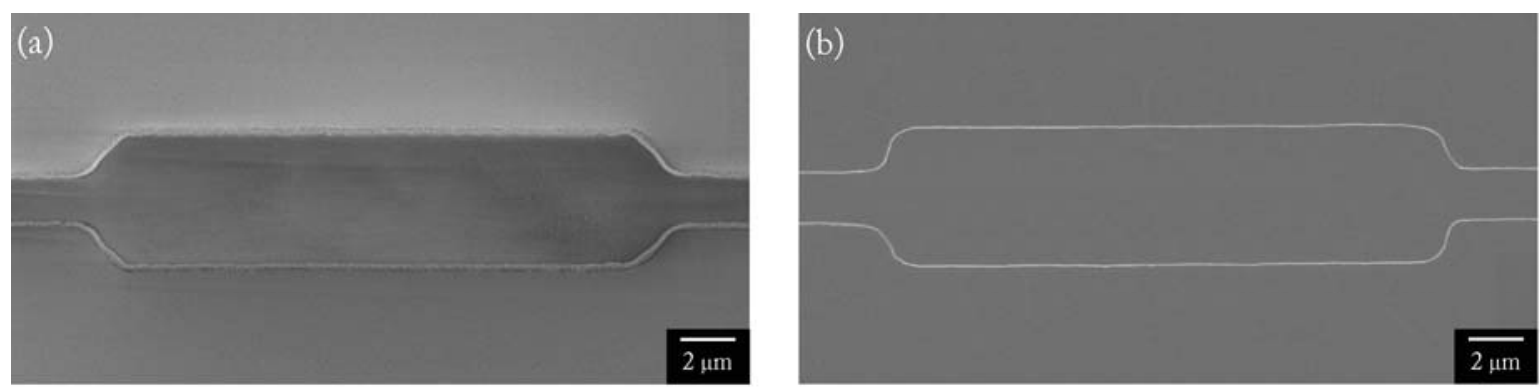

Figure 7.2 SEM images of the waveguide outline structures at two different stages of the fabrication procedure: (a) after exposure and development of Olin 907 and (b) after anisotropic etching into silicon.

The most important parameter for consideration in this fabrication procedure is the type of etch technique to transfer photoresist waveguide patterns into silicon as any surface roughness could potentially cause detrimental losses for light propagation through the resulting $2 \mathrm{D} \mathrm{PhCWGs.}{ }^{[23]}$ One potential silicon etch technique that is known to result in ultralow surface roughness is the anisotropic wet-chemical etching of silicon using aqueous basic solutions like commercially available resist developer OPD 4262. ${ }^{[24]}$ According to this study, OPD 4262 was superior to other aqueous basic etch solutions with respect to surface finish (surface roughness, $R_{a}<0.5 \mathrm{~nm}$ ). At room temperature, a silicon etch rate in the $\langle 110\rangle$ direction, i.e., the direction perpendicular to the wafer surface of the $\langle 110\rangle$ silicon wafer, of $3.7 \mathrm{~nm} \cdot \mathrm{min}^{-1}$ was found along with an excellent etch uniformity $( \pm 1 \mathrm{~nm})$ over the entire 4-inch wafer and a selectivity of (at least) 250 with respect to $\mathrm{SiO}_{2}$ as the etch mask. ${ }^{[25]}$ As a result of the high etch selectivity, native $\mathrm{SiO}_{2}$ was found to be a suitable etch mask in the present procedure to anisotropically etch the resist patterns into the top silicon layer of 
the SOI substrate in OPD 4262. The resist patterns were transferred into native $\mathrm{SiO}_{2}$ by etching in 1\% HF (Scheme 7.1b). One notable finding in initial etch experiments was that some commercial photoresists are incompatible with $1 \%$ HF (e.g., image reversal resist Ti09 XR (MicroChemicals) is in positive mode only compatible with 1\% HF after additional UV crosslinking), resulting in a failure to etch the waveguide outline structures into silicon in the next step due to the complete removal of the native $\mathrm{SiO}_{2}$. After resist removal in acetone and cleaning of the wafer in isopropanol, the waveguide outline structures were transferred $100 \mathrm{~nm}$ into the silicon wafer by wet-chemical etching in OPD 4262 using the native $\mathrm{SiO}_{2}$ as etch mask (Fig. 7.2b, Scheme 7.1c). At the correct alignment of the waveguide structures on the $<110>$ silicon wafer, anisotropic wet-chemical etching occurs in the direction perpendicular to the wafer surface resulting in waveguide structures with perfect vertical sidewalls. After a second standard cleaning procedure, the photonic crystal structures were written into the resulting silicon waveguide outline structures of the silicon mold by FIB milling (Scheme 7.1d). The final silicon mold, consisting of narrow (W0.7) line-defect 2D PhCWGs with a total length of about $22 \mu \mathrm{m}$ (or $56 a$ ) in between $2 \mu \mathrm{m}$-wide ridge waveguides, is shown in Fig. 7.3.

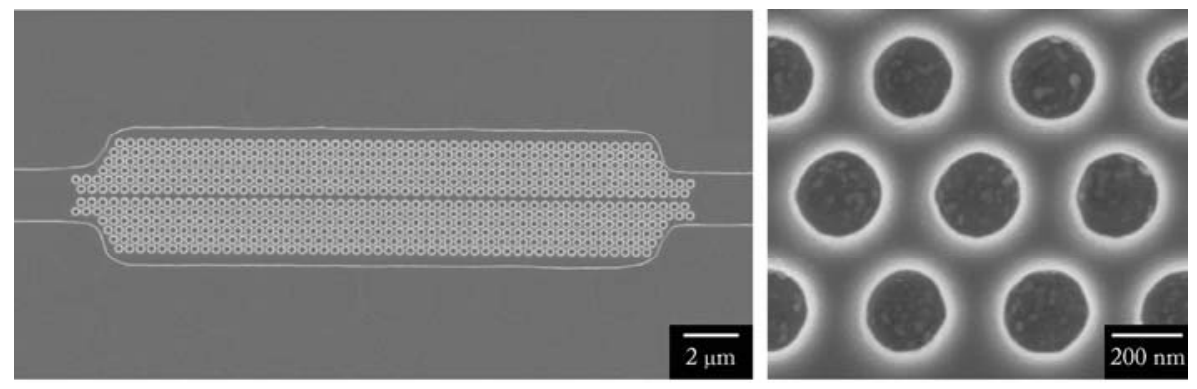

Figure 7.3 SEM images of the final NIL mold after FIB writing of the photonic element into the waveguide outline structures $(d=228 \pm 4 \mathrm{~nm}, a=396 \pm 6 \mathrm{~nm})$. $^{[26]}$

Several initial milling tests were done to determine (i) the offset after loading and positioning of the stream file pattern with the actual milling position in the patterning field and (ii) the magnification that matches the total lateral dimensions of the resulting photonic pattern to the dimensions set in the stream file (see Experimental). Accurate positioning and milling of the photonic elements within the silicon waveguide outline structures was the result after correction for the offset at positioning 
of the stream file pattern onto the target section within the ridge waveguides. In addition to accurate positioning, the close-up of Fig. 7.3 illustrates the excellent circular definition of the photonic elements. Feature dimension analysis of the silicon mold illustrates that the feature diameter was about $14 \mathrm{~nm}$ larger than the design geometry of the PhCWG (Section 7.2.1). Since the periodicity conforms to the design setting, some plausible causes for only the diameter to deviate from the design setting could reside in the current beam overlap ${ }^{[27]}$ and the feature definitions in the stream file. ${ }^{[28]}$

\subsubsection{Replication of 2D PhCWGs}

The inverse imprinting procedure to replicate the 2D PhCWGs into SOI is shown in Scheme 7.2.
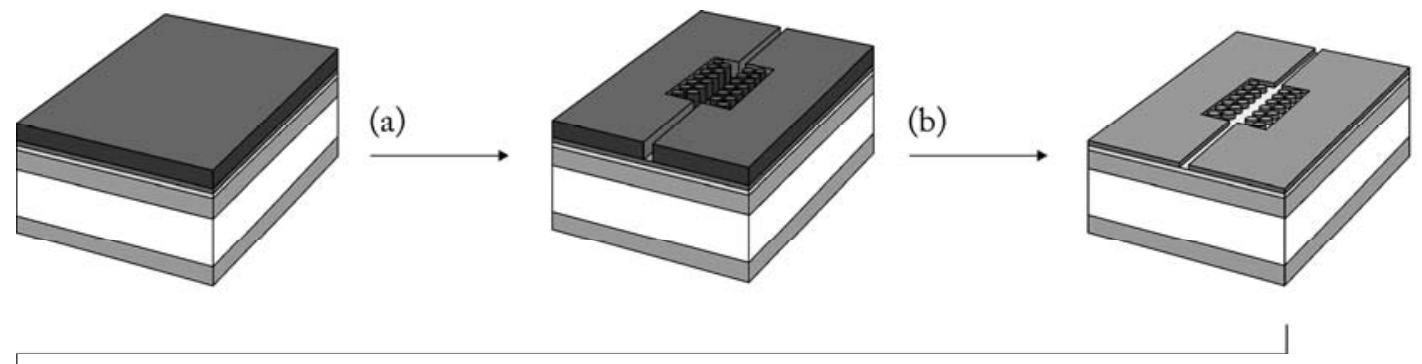

(c)

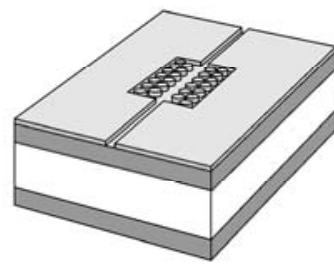

$<100>\operatorname{silicon}(315 \mathrm{~nm})$

PMMA (110 nm) (d)

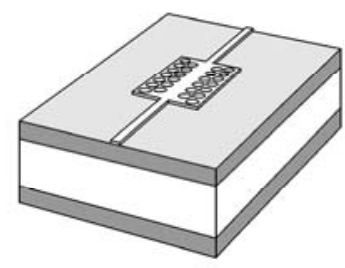

(e)

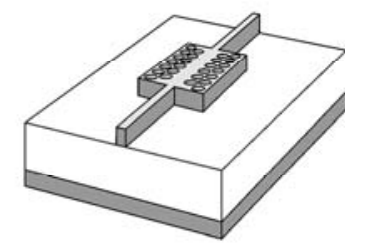

$\mathrm{Si}_{3} \mathrm{~N}_{4}(14 \mathrm{~nm})$

LPCVD-SiO $2(12 \mathrm{~nm})$

thermal $\mathrm{SiO}_{2}(2 \mu \mathrm{m}, 12 \mathrm{~nm})$

Scheme 7.2 Schematic representation of the lithographic procedure for the replication of the $2 \mathrm{D}$ PhCWGs on SOI substrates: (a) thermal NIL in PMMA (and removal of the residual layer by $\mathrm{O}_{2}$ plasma, (b) wet-chemical etching of $\mathrm{LPCVD}-\mathrm{SiO}_{2}$ using the PMMA imprint as the etch mask, and removal of PMMA, (c) wet-chemical etching of $\mathrm{Si}_{3} \mathrm{~N}_{4}$ and removal of the LPCVD SiO 2 etch mask, (d) dry oxidation of silicon and removal of the $\mathrm{Si}_{3} \mathrm{~N}_{4}$ oxidation, and (e) cryogenic etching of the final 2D PhCWGs in the top silicon layer of the SOI substrate. 
The process for the replication of $2 \mathrm{D}$ PhCWGs into SOI elaborates on the LOCOS inversion procedure and design rules set in Chapter 6 by applying this procedure to polymer imprint patterns of 2D PhCWGs by thermal NIL. Substrate preparation was done in order to allow for accurate pattern transfer: (i) deposition of two ultrathin transfer layers by low-pressure chemical vapor deposition (LPCVD) (in the order: SOI substrate, silicon nitride $\left(\mathrm{Si}_{3} \mathrm{~N}_{4}, 14 \mathrm{~nm}\right), \mathrm{LPCVD}-\mathrm{SiO}_{2},(12 \mathrm{~nm})$ ) and (ii) hightemperature annealing of the substrate under dry nitrogen to facilitate accurate pattern transfer during the wet-chemical etching of these two transfer layers in terms of resolution, etch rates, and etch selectivity.

\subsubsection{Thermal nanoimprint lithography}

Prior to application in thermal NIL, a second standard cleaning procedure was applied to the silicon mold in combination with a dehydration bake. Subsequently, a monolayer of perfluorodecyltrichlorosilane was grown onto the molds by gas phase deposition under vacuum in a desiccator to provide the molds with anti-adhesion properties. Spin-coating of thermoplastic polymers was done on the substrate at a thickness of $\sim 110 \mathrm{~nm}$. Patterning of the first transfer layer $\left(\mathrm{LPCVD}-\mathrm{SiO}_{2}\right)$ in 1\% HF using such thin polymer etch masks essentially requires HF-impermeable polymers (or high $\mathrm{LPCVD}-\mathrm{SiO}_{2}$ etch rates) in order to exploit the intrinsic high etch selectivity in this etch step. Patterns of PMMA $\left(\mathrm{M}_{\mathrm{w}}=350 \mathrm{kDa}\right)$ and polystyrene (PS, $M_{w}=240 \mathrm{kDa}$ ) were only partially resistant to $\mathrm{HF}$, resulting in gradual dissolution of the $\mathrm{LPCVD}-\mathrm{SiO}_{2}$ and detachment of the polymer imprint patterns from the substrate in time (< $60 \mathrm{~s})$. High-molecular-weight PMMA $\left(\mathrm{M}_{\mathrm{w}}=996 \mathrm{kDa}\right)$ was found to resist $1 \% \mathrm{HF}$ for at least $2 \mathrm{~min}$ and was therefore the polymer of choice in the NIL replication step.

For imprinting of the 2D PhCWG structures into this type of PMMA (Scheme 7.2a), the conditions for imprinting were set to a temperature $\sim 80^{\circ} \mathrm{C}$ above the glass-transition temperature and a pressure of 50 bar during an imprint time of 2 min. This procedure could be repeated for several tens of imprints without any observable deterioration of the silicon mold. Feature dimension analysis of the resulting 2D PhCWG structures in PMMA and comparison to the feature dimensions 
of the NIL mold illustrate the high accuracy (deviation in lateral feature dimensions $\sim 4 \mathrm{~nm}$ ) of thermal NIL to replicate the photonic structures into PMMA (Fig. 7.4).

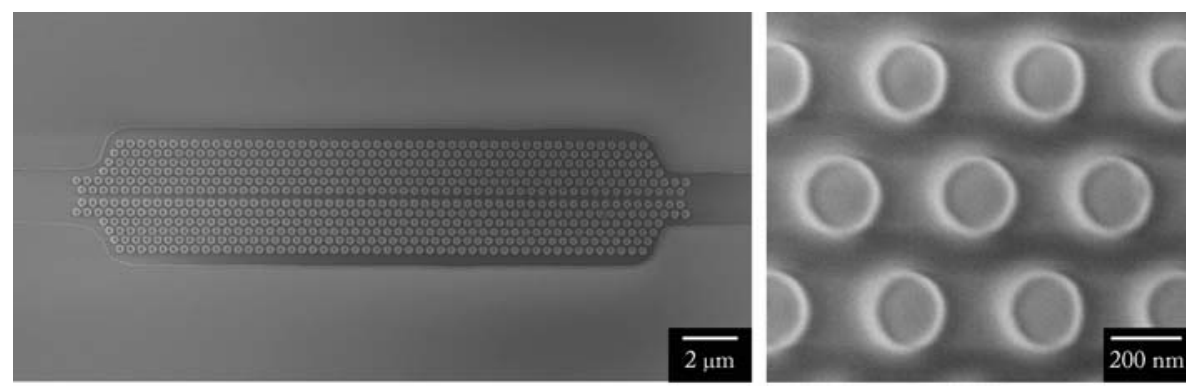

Figure 7.4 SEM images of the PMMA imprint before removal of the residual layer by $\mathrm{O}_{2}$ plasma $(d=232 \pm 4 \mathrm{~nm}, a=394 \pm 6 \mathrm{~nm}) .{ }^{[26]}$

The residual layer was kept as thin as possible by spin-coating PMMA layers that are close in thickness to the imprint depth of the NIL mold. At the very low pattern density of the current NIL mold $(<1 \%)$, the difference between the polymer layer thickness and the imprint depth of the mold was taken (to a first approximation) as the thickness of the residual layer. Removal of this intrinsically thin residual polymer layer was achieved by anisotropic $\mathrm{O}_{2}$ RIE to preserve the lateral dimensions of the PMMA features and, as a result, the accuracy of the present replication process. Temperature control over the electrode was excellent, resulting in a good etch uniformity at an intrinsic etch rate of $8 \mathrm{~nm} \cdot \mathrm{min}^{-1}$ (at 100\% PMMA loading) over the entire 4-inch wafer (deviation in layer thickness $<2 \mathrm{~nm}$ ). Substantial over-etching (on the order of $100 \%$ with respect to the theoretical etch time) was chosen to assure complete removal of the residual layer and therefore accurate transfer of the imprint pattern into the underlying $\mathrm{LPCVD}-\mathrm{SiO}_{2}$ transfer layer. The $\mathrm{O}_{2}$ plasma had no significant effect on the surface roughness, as observed by AFM (data not shown) and as was shown before. ${ }^{[29]}$ For process considerations, the only important side effect of the $\mathrm{O}_{2}$ plasma treatment is the increase in the hydrophilicity of the PMMA layer. This effect could facilitate etching of the first pattern transfer layer, i.e., $\mathrm{LPCVD}-\mathrm{SiO}_{2}$, in the aqueous etch solution using PMMA as the etch mask. 


\subsubsection{LOCOS inversion procedure}

After the removal of the residual layer by $\mathrm{O}_{2}$ plasma, the PMMA imprint was transferred into LPCVD- $\mathrm{SiO}_{2}$ by etching in $1 \% \mathrm{HF}$ (Scheme 7.2b). Subsequent to stripping the PMMA in acetone/isopropanol, the $\mathrm{LPCVD}-\mathrm{SiO}_{2}$ pattern was transferred into $\mathrm{Si}_{3} \mathrm{~N}_{4}$ by etching in $85 \% \mathrm{H}_{3} \mathrm{PO}_{4}$ at $180{ }^{\circ} \mathrm{C}$ (Scheme 7.2c). The resulting $\mathrm{Si}_{3} \mathrm{~N}_{4}$ oxidation mask for LOCOS is shown in Fig. 7.5a (after stripping the $\mathrm{LPCVD}-\mathrm{SiO}_{2}$ in $\left.1 \% \mathrm{HF}\right)$.
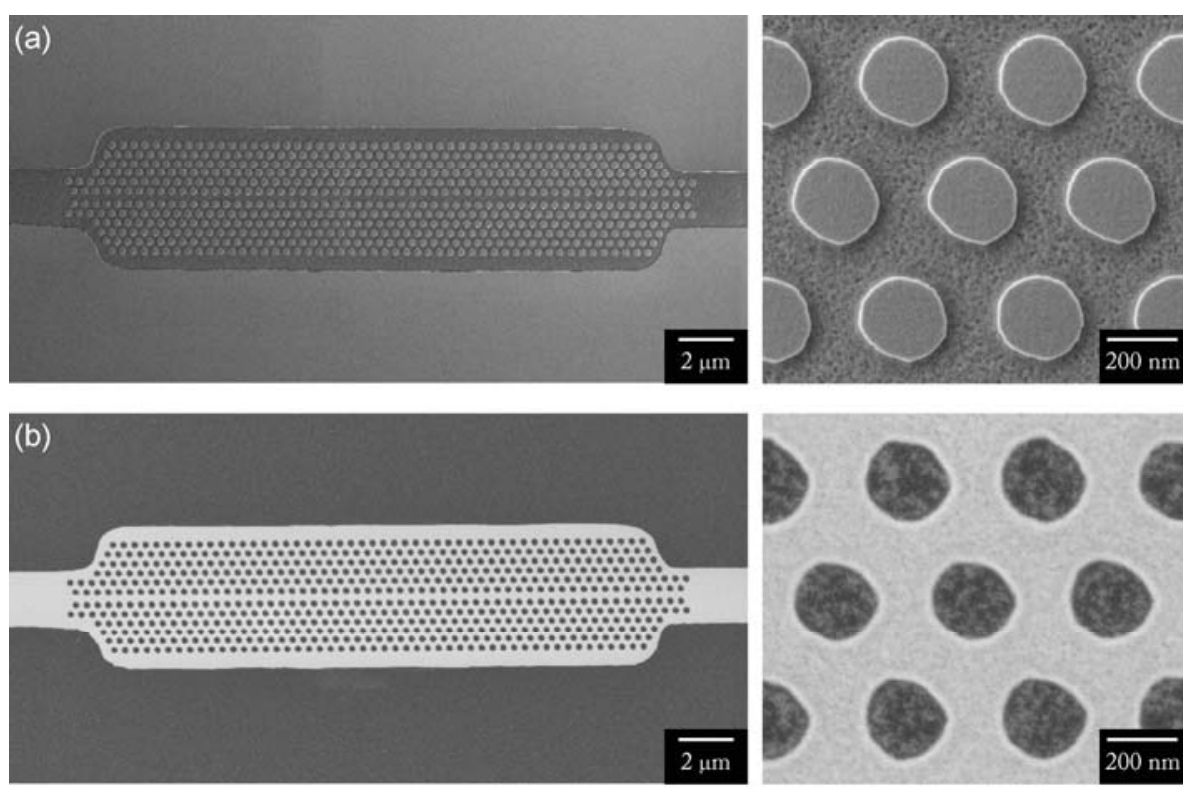

Figure 7.5 SEM images of two different stages in the pattern inversion process: (a) after successive transfer of the imprint pattern into $\mathrm{Si}_{3} \mathrm{~N}_{4}(d=252 \pm 2 \mathrm{~nm}, a=396 \pm 4 \mathrm{~nm})$ and (b) after local oxidation of silicon into thermal $\mathrm{SiO}_{2}(d=232 \pm 2 \mathrm{~nm}, a=394 \pm 4 \mathrm{~nm}) \cdot{ }^{[26]}$

Feature dimension analysis of the $\mathrm{Si}_{3} \mathrm{~N}_{4}$ oxidation mask illustrates a total increase of $20 \mathrm{~nm}$ in feature dimensions during the successive transfer of the PMMA imprint pattern into the underlying two transfer layers. Finally, the SOI substrates were put in a dry oxidation furnace at $950{ }^{\circ} \mathrm{C}$ for $12 \mathrm{~min}$ to perform the pattern inversion by LOCOS using the ultrathin $\mathrm{Si}_{3} \mathrm{~N}_{4}$ patterns as oxidation masks (Scheme 7.2d). The resulting thermal $\mathrm{SiO}_{2}$ etch mask after stripping the $\mathrm{Si}_{3} \mathrm{~N}_{4}$ in $85 \% \mathrm{H}_{3} \mathrm{PO}_{4}$ at $180{ }^{\circ} \mathrm{C}$ is shown in Fig. 7.5b. Feature dimension analysis of the thermal $\mathrm{SiO}_{2}$ etch mask illustrates that the well-known lateral encroachment of thermal $\mathrm{SiO}_{2}$ under the $\mathrm{Si}_{3} \mathrm{~N}_{4}$ mask during LOCOS effectively was capable of cancelling the $20 \mathrm{~nm}$ increase in 
feature dimensions during the preceding etch steps of the transfer layers. The pattern transfer accuracy at this stage of the replication procedure is excellent with a deviation in lateral feature dimensions of $<4 \mathrm{~nm}$.

\subsubsection{Anisotropic etching of waveguide structures into SOI}

The last step in the replication of the 2D PhCWGs was the anisotropic etching into SOI at cryogenic temperatures (Scheme 7.2e). Etching photonic structures through $\mathrm{SiO}_{2} / \mathrm{Si} / \mathrm{SiO}_{2}$ substrates in the micrometer range has been shown with this apparatus in previous studies. ${ }^{[30]}$ Additionally, for accurate etching of smaller SOI substrates $(18 \mathrm{~mm} \times 18 \mathrm{~mm})$, efficient thermal contact of the SOI substrate with the substrate electrode of the etch apparatus was achieved by mounting the SOI substrate onto a 4 -inch substrate silicon wafer with a thin layer of vacuum grease in between the two surfaces. Just before mounting, the native oxide on the substrate silicon wafer was removed in $1 \% \mathrm{HF}$ in order to attain close to $100 \%$ silicon loading (as in the case of the test experiments).

At a silicon etch rate of $\sim 600 \mathrm{~nm} \cdot \mathrm{min}^{-1}$ and an etch selectivity of silicon over thermal $\mathrm{SiO}_{2}$ of $\sim 50$ (see Chapter 6, Section 6.2.2), the current anisotropic etch recipe allows for accurate etching with sufficient latitude to intercept the effect of RIE lag. RIE lag is the aspect ratio-dependent etching that occurs during the simultaneous etching of different features sizes due to ion depletion and redeposition. ${ }^{[31]}$ The magnitude of RIE lag in the etching of the present PhCWG, i.e., the $300 \mu \mathrm{m}$ interspaces between the waveguides and the $\sim 230 \mathrm{~nm}$ holes of the photonic element, was $\sim 5 \%$ according to cross-section examination of a substrate by high-resolution SEM. Some over-etching (on the order of $20 \%$ with respect to the theoretical etch time) was done to ensure complete transfer into the top silicon layer, with the $\mathrm{SiO}_{2}$ layer acting as an etch stop.

Feature dimension analysis of the final 2D PhCWG on SOI by SEM (Fig. 7.6, after stripping the thermal $\mathrm{SiO}_{2}$ etch mask in $1 \% \mathrm{HF}$ ) illustrates that overall pattern transfer accuracy the $2 \mathrm{D}$ PhCWGs on SOI by the inverse imprinting procedure is competitive in terms of feature lateral dimensions (within $4 \mathrm{~nm}$ in comparison to the dimensions of the silicon mold) and feature definition to the accuracy of state-of-theart nanolithographic techniques, e.g., direct e-beam lithography and focused ion-beam 
lithography. Additionally, the current critical feature dimension of NIL of $5 \mathrm{~nm}^{[32]}$ implies that the present inversion procedure could also become competitive in terms of pattern resolution capability.
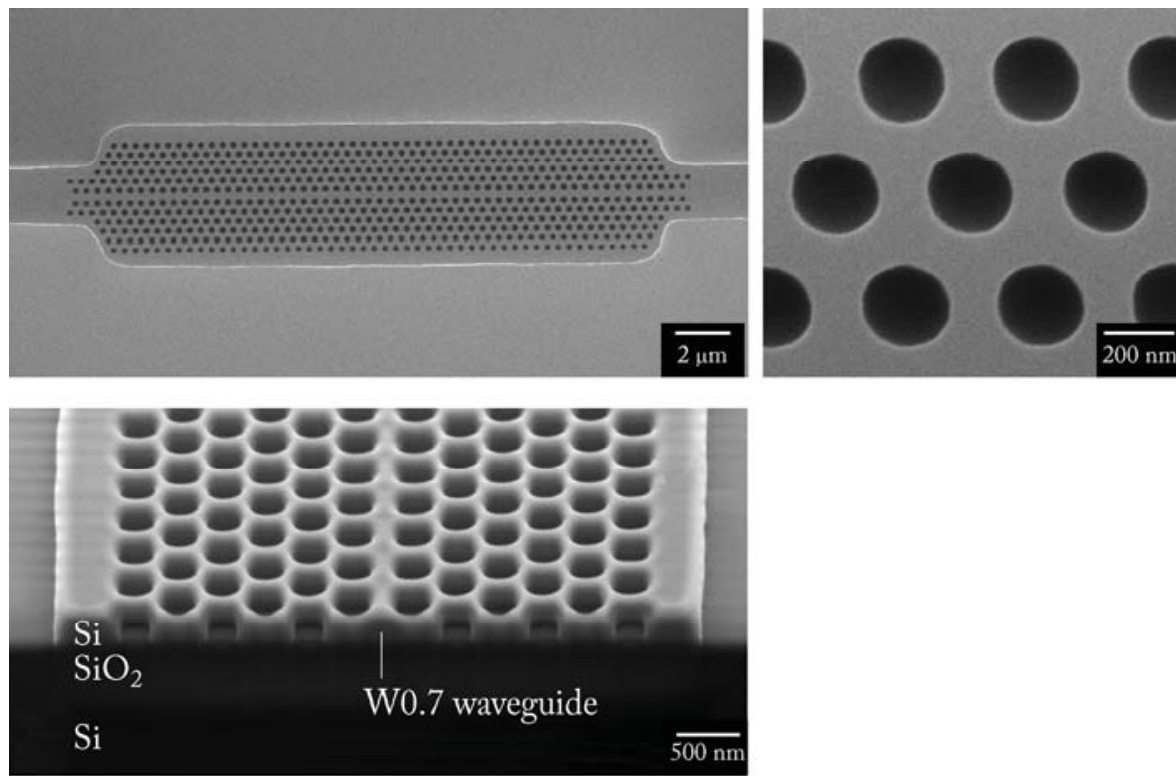

Figure 7.6 SEM images of the final 2D PhCWG on SOI $(d=228 \pm 2 \mathrm{~nm}, a=392 \pm 4 \mathrm{~nm})$.

SEM cross-section analysis in Fig. 7.6 illustrates the merits of the systematic etch procedure (Chapter 6) in achieving anisotropic etching at high etch selectivity of silicon over thermal $\mathrm{SiO}_{2}$.

\subsubsection{Optical characterization by PSTM}

Photon scanning tunneling microscopy (PSTM) ${ }^{[33]}$ was exploited to investigate the optical quality of the present $2 \mathrm{D}$ PhCWGs, as this technique has the capacity to investigate the propagation of light inside the line defect of 2D PhCWGs with subwavelength resolution. ${ }^{[34]}$ The phase-sensitive PSTM setup consists of a collection mode scanning near-field optical microscope (SNOM) in combination with a MachZehnder interferometer. A sensitive (photon) detector measures the interference signal (from the interferometer) between the reference signal and the signal from the nearfield probe. The lock-in amplifier finally processes the detector signal and allows the simultaneous measurement of the local amplitude and phase of the electromagnetic field propagating inside a 2D PhCWG. Additionally, the electronic feedback system 
keeps the probe at a constant height $(\sim 20 \mathrm{~nm})$ above the $2 \mathrm{D}$ PhCWG and enables the correlation of the light intensity distribution to the resulting topographical image of the 2D PhCWG (see Fig. 7.7 for a schematic representation).

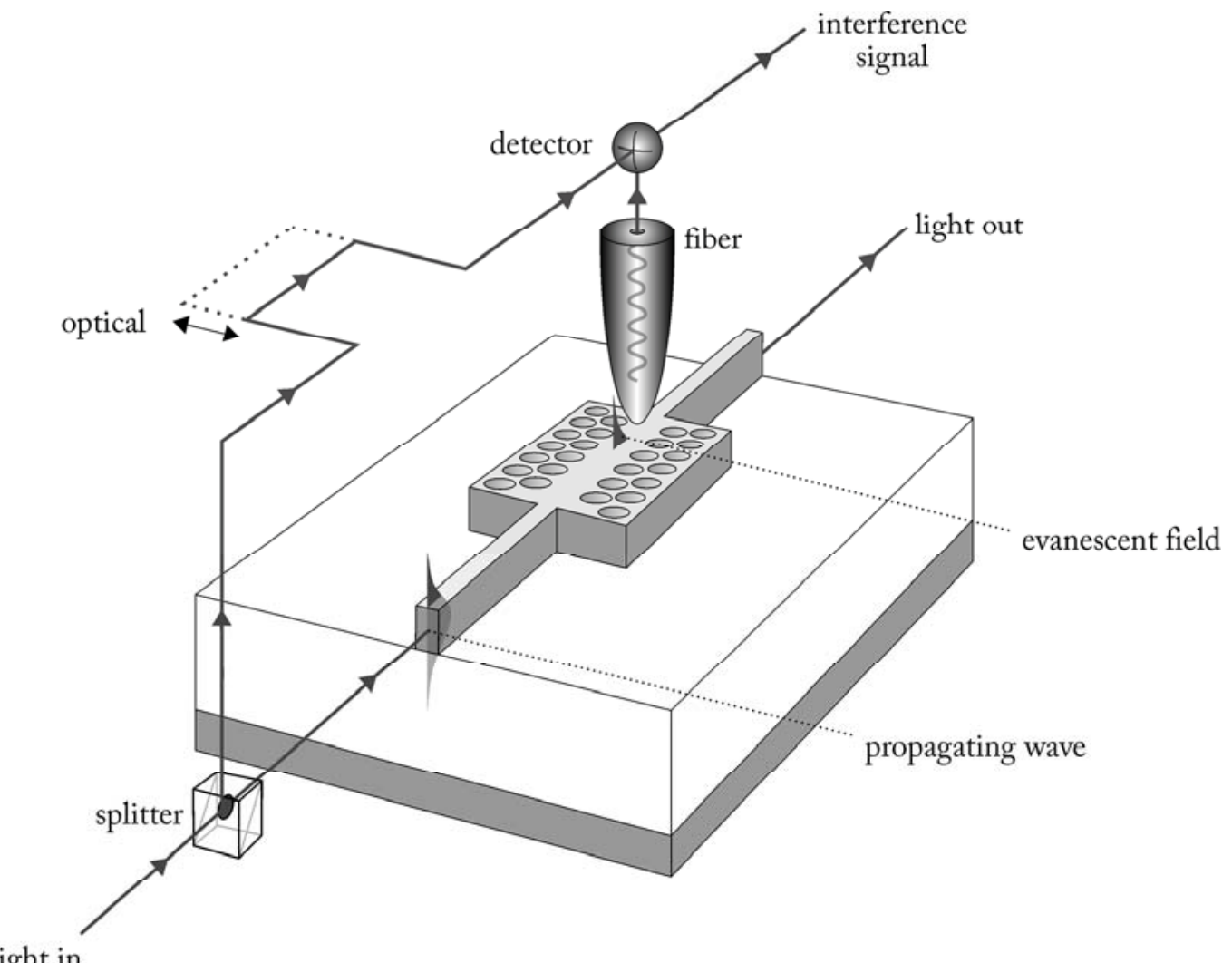

light in

Figure 7.7 Schematic representation of the PSTM setup. The light source is a tunable laser (HP 8168F by Atecorp $\left.{ }^{[35]}\right)$, operating in the wavelength range of $1440-1592 \mathrm{~nm}$, and is capable of delivering laser light of a specific polarization to the $2 \mathrm{D}$ PhCWG through a single-mode fiber (SMF-28e(R) by Corning ${ }^{[36]}$ ). A nanoscopic near-field probe senses the evanescent field of the propagating light by coupling of this field to the modes of the probe, while a piezo actuator enables raster scanning of a probe over the 2D PhCWG (max. scan size $200 \times 200 \mu \mathrm{m}^{2}$ ) in order to obtain a local intensity distribution of the light inside the $2 \mathrm{D} \mathrm{PhCWG}$ at sub-wavelength resolution.

Recently, slow light propagation in 2D PhCWGs has been shown by exploiting the scanning near-field optical microscope. ${ }^{[37]}$ The visualization of the optical propagation allows a direct 'eye-balling' of scattering at imperfections. Additionally, loss measurements on one structure are feasible, without requiring multiple structures for a (virtual) cut-back measurement. ${ }^{[38]}$ Here, advantage of the phase-sensitive PSTM was taken to elucidate the dispersion properties of $2 \mathrm{D}$ PhCWGs by measuring the local 
amplitude and phase of the light. In this way the accuracy in the periodicity of the crystal becomes accessible.

\subsubsection{Amplitude analysis of the $2 \mathrm{DPhCWG}$}

Fig. 7.8 illustrates the outcome of a typical PSTM measurement on our 2D PhCWGs.
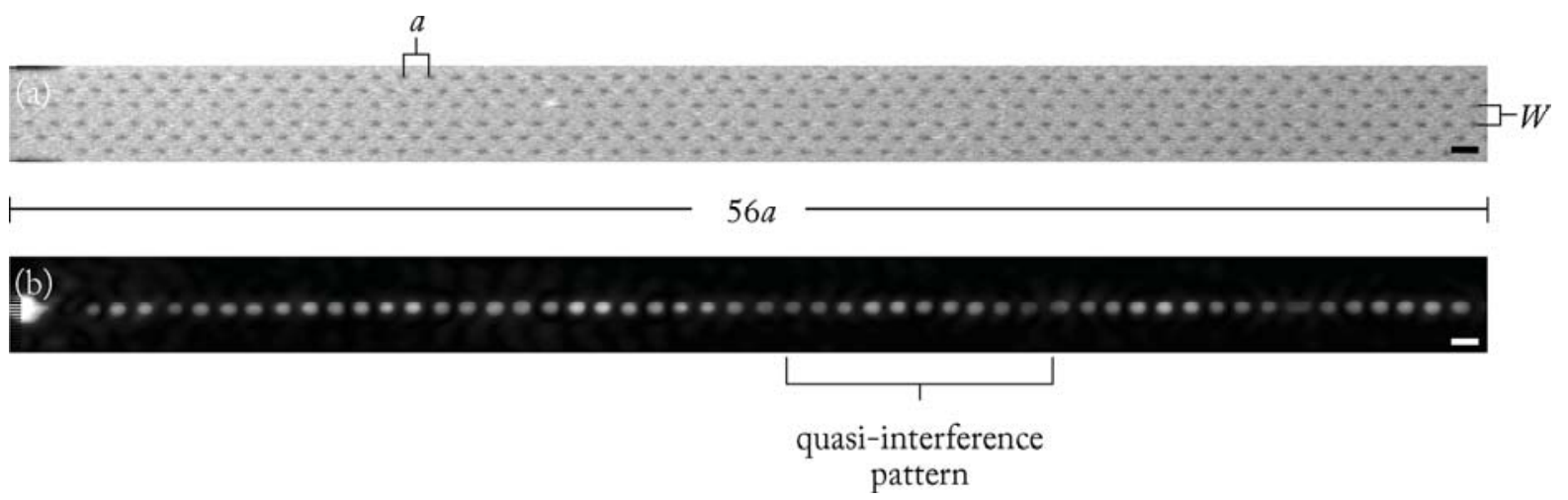

Figure 7.8 Phase-sensitive PSTM measurement of the 2D PhCWG under investigation: (a) topographical image of the 2D PhCWG revealing the triangular arrangement of the air holes; (b) optical field distribution (amplitude) image of the propagating light $\left(\lambda_{\circ}=1575 \mathrm{~nm}\right)$. The light is incident into the $2 \mathrm{D} \mathrm{PhCWG}$ from the left. The scale bar in the images denotes a lateral dimension of $400 \mathrm{~nm}$.

The triangular lattice of the air holes $(a)$ as well as the narrow line defect of the 2D PhCWG ( $W=472 \mathrm{~nm})$ are clearly resolved in the topographical image (Fig. 7.8b). The optical amplitude image (Fig. 7.8b) reveals that the propagating light is highly confined to the line defect in the $2 \mathrm{D} \mathrm{PhC}$; the amplitude outside the defect is more than 1 order of magnitude smaller at a distance equal to a single lattice unit. Apart from the high amplitude at the transition between the access waveguide and the 2D PhCWG, no high amplitude spikes are present, which would have revealed scattering at local imperfections. On closer examination, a periodicity is apparent in the amplitude signal which is equal to the lattice constant of the $2 \mathrm{D} \mathrm{PhCWG}$. This periodicity was found to remain constant irrespective of the excitation frequency of the light. ${ }^{[39]}$ This is a clear manifestation of the fact that the light propagating in the periodic waveguide is governed by Bloch's theorem, ${ }^{[40]}$ which is indicative of a high definition of the periodicity of the 2D PhCWG. Another striking feature, albeit faint, in the amplitude image is the presence of a larger periodic modulation along the 
waveguide with a periodicity of about $10 a$ (or $\sim 4 \mathrm{~m}$ ) as indicated in Fig. 7.8b. This feature can be attributed to the quasi-interference between TE-like and TM-like modes. $^{[40]}$

Phase-sensitive PSTM also enables the quantification of the propagation losses of the light inside a single line defect. By selecting specific modes in reciprocal space, one can determine the losses of only the mode of interest, i.e., the TE mode. By measuring the decay of the amplitude directly as a function of position, the need for investigating other reference structures is not necessary and the measurement is insensitive to the potential influence of coupling losses (the so-called insertion losses) at the interface of the access ridge waveguide and the 2D PhCWG. Fig. 7.9a illustrates the intensity plot for light of TE polarization along the line defect of the $22 \mu \mathrm{m}$ long $2 \mathrm{D}$ PhCWG at a specific frequency $\left(\lambda_{\circ}=1575 \mathrm{~nm}\right)$.

(a)

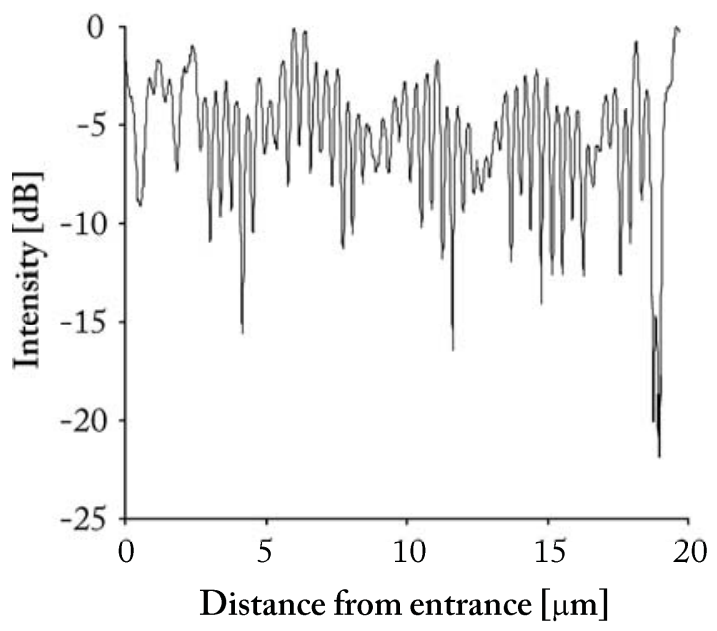

(b) $\omega(2 \pi(\mathrm{c} / \mathrm{a}))$

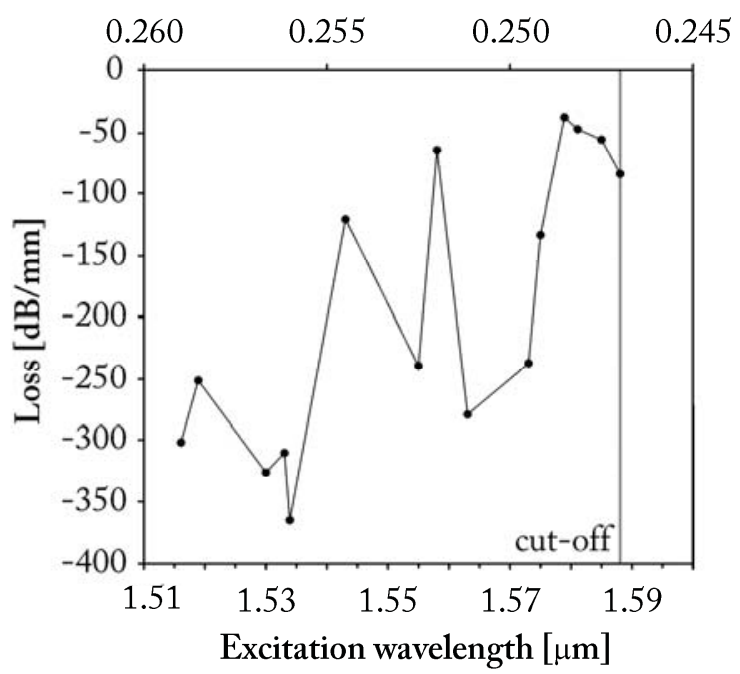

Figure 7.9 Propagation loss in the 2D PhCWG. (a) Intensity profile of light $\left(\lambda_{o}=1575 \mathrm{~nm}\right)$ in the center of the line defect as a function of the distance from the entrance. (b) Propagation loss as a function of the wavelength over the frequency range of the tunable laser up to the cut-off frequency.

The propagation losses for light of TE polarization were determined at different excitation wavelengths (see Fig. 7.9b) from a linear fit of the intensity profiles analogous to Fig. 7.9a, assuming that the interaction between the probe and the waveguide remains constant over the entire scan area. Below the cut-off frequency, the penetration length of the light inside the line defect of the $2 \mathrm{D}$ PhCWG was found to 
be $\sim 2 \mu \mathrm{m}$. For frequencies above the cut-off frequency and below the $\mathrm{SiO}_{2}$ light line, the forward propagating mode was selected in reciprocal space ${ }^{[41]}$ and, from its decay as a function of position, losses down to $36 \mathrm{~dB} \cdot \mathrm{mm}^{-1}$ were found close to the cut-off frequency. ${ }^{[42]}$ By selecting the backward propagating mode, it was found that the loss induced by reflection (due to a combination of small defects inside the photonic structure and insertion loss at the end of the photonic crystal waveguide and the start of the exiting ridge waveguide) is only $4 \%$. The main source of losses is due to out-ofplane scattering. While these losses are higher than the reported propagation losses by Notomi, ${ }^{[19 a]}$ these results are highly encouraging. Firstly, because our measurements represent the first loss measurements on high-index photonic crystal waveguides fabricated with NIL. Secondly, because it is anticipated that tuning the silicon slab thickness from the $0.8 a$ in the present $2 \mathrm{D}$ PhCWGs to the optimal slab thickness of $0.4-0.5 a^{[43]}$ and transforming into an air-bridge type waveguide should significantly reduce the propagation losses.

\subsubsection{Band structure analysis of the $2 D P h C W G$}

From the phase information of the PSTM, it is possible to study the Bloch nature of the light in the waveguide. Fourier transformation enables the determination of the spatial frequencies of the propagating light: the wavevectors. By repeating this procedure as a function of the optical frequency through tuning of the narrow bandwidth tunable laser, the photonic band diagram of the 2D PhCWG was measured in the excitation wavelength range from $1496 \mathrm{~nm}(\omega=0.262)$ up to $1592 \mathrm{~nm}(\omega=0.246) \cdot{ }^{[44]}$ Fig. 7.10 illustrates the experimental band diagram of the 2D PhCWG in the excitation wavelength range from $1440 \mathrm{~nm}(\omega=0.272)$ up to $1592 \mathrm{~nm}(\omega=0.246)$ after plotting the frequency against the resulting $k$ vectors. Several separate photonic modes are apparent in Fig. 7.10: the fundamental TE-like mode and the TM-like modes, as indicated by the white and black arrows, respectively, propagating in forward direction. That these modes are found at spatial frequencies $k$ and $k-2 / a$ is a direct proof that the light propagating inside the 2D PhCWG experiences a periodic modulation of the dielectric constant and a confirmation of the phase analysis concerning the periodic amplitude modulation (Section 7.2.4.1, Fig. 7.8b). The TE-like mode bends towards the edge of the 
Brillouin zone $(k=\pi / a)$, near $\omega=0.245$. Apart from a slight shift of $\Delta \omega=0.005$, the measured photonic band structure is in qualitative agreement with the calculations of Notomi. ${ }^{[19]}$

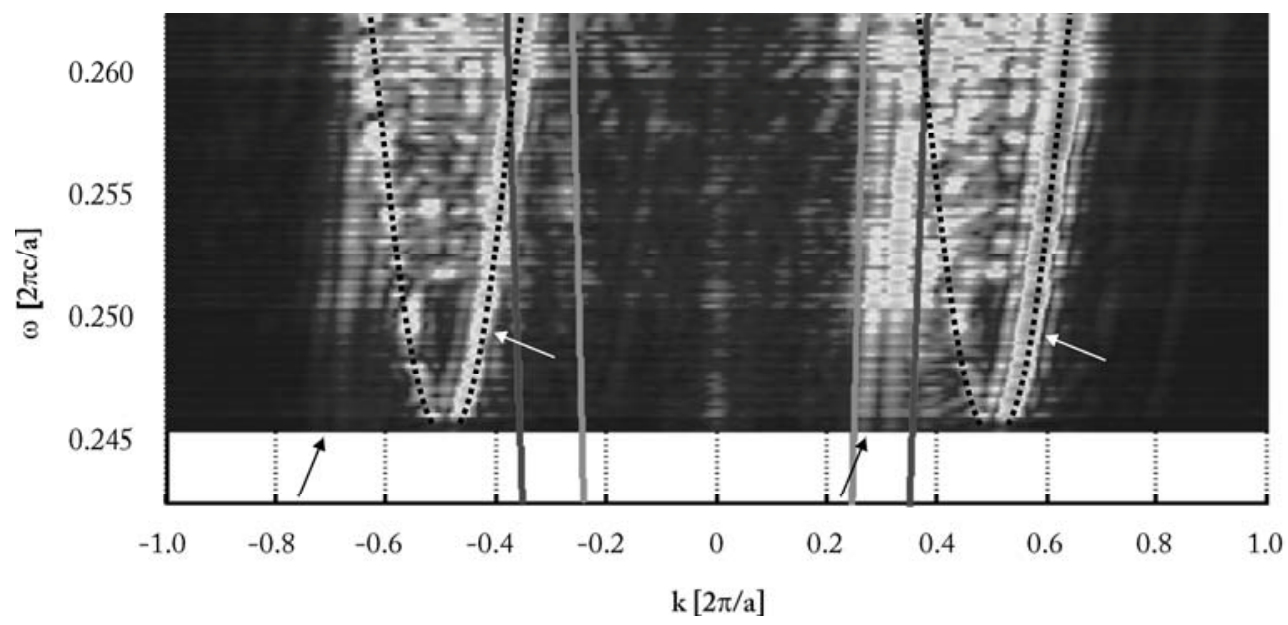

Figure 7.10 Experimentally determined photonic band diagram of the 2D PhCWG. The arrows in the band diagram indicate the dispersion curves of different modes of the waveguide: the dispersion curves indicated with the white arrows are both part of the TE-like mode and the curves indicated by the black arrows both belong to the TM-like mode. The light gray and dark grey lines are the light lines in vacuum and in $\mathrm{SiO}_{2}$, respectively. The black dotted lines serve as a guide to the eye for the TE mode.

A strong dispersion is apparent at the Brillouin zone edge leading to slow light between $\lambda_{\circ} 1575 \mathrm{~nm}(\omega=0.249)$ and $1590 \mathrm{~nm}(\omega=0.247)$. The measured photonic band structure reflects only a minimal amount of light propagating in the backward direction. This observation again attests to the high quality of the present 2D PhCWG.

\subsection{Conclusions}

This chapter demonstrates the entire lithographic procedure for the fabrication and replication of $2 \mathrm{D}$ PhCWGs on $\mathrm{SIO}$ substrates. The combination of conventional photolithography with local FIB writing was shown to result in fast prototyping of silicon masters containing 2D PhCWGs with ultra-smooth sidewalls by incorporating anisotropic wet-chemical etching of silicon in OPD 4262 into the fabrication procedure. 
The combination of thermal NIL, LOCOS and cryogenic RIE is very accurate in terms of pattern transfer accuracy (deviation in lateral dimension of $<4 \mathrm{~nm}$ ) and feature definition of the final 2D PhCWG on SOI owing to the use the lithographic procedures in Chapter 6. This process shows the highest pattern transfer accuracy to date in the replication of $2 \mathrm{D}$ PhCWGs into SOI by NIL and is therefore a viable alternative nanofabrication technique for nanophotonics.

The present 2D PhCWG exhibits excellent optical characteristics, as shown by near-field microscopy, by illustrating good confinement of propagating light in the line defect at wavelengths in the telecommunications range with encouragingly low losses.

\subsection{Experimental}

Chemicals and materials: All experiments except the nanoimprint experiments were carried out in cleanroom environment. All chemicals and materials were purchased from commercial resources: 4-inch DSP <110> p-type silicon wafers (Okmatic), 8-inch SOI wafers (SOITEC, UNIBOND ${ }^{\mathrm{TM}}$ : $340 \mathrm{~nm}$ silicon on $2000 \mathrm{~nm} \mathrm{SiO}_{2}$ ), Olin 907 (Fujifilm Arch), hexamethyldisilazane (HMDS) (100\% Selectipur: Merck), OPD 4262 resist developer (Arch Chemicals), $\mathrm{HNO}_{3}$ (100\% Selectipur: Merck, and 69\% VLSI: Merck), HF (1\% VLSI: Merck), $\mathrm{H}_{3} \mathrm{PO}_{4}$ (85\% VLSI, Merck), acetone and isopropylalcohol (Merck, VLSI), PMMA (Aldrich), toluene (Acros), $1 H, 1 H, 2 H, 2 H-$ perfluorodecyltrichlorosilane (ABCR), and vacuum grease (Crawford fitting company).

Imprint mold fabrication: the outline structures of the 2D PhCWGs on the mold were prepared by conventional optical lithography (ElectroVisions Group mask aligner (EVG), model EV620). <110> DSP silicon wafers were cleaned by a standard cleaning procedure $\left(10 \mathrm{~min}\right.$ in $70 \% \mathrm{HNO}_{3}, 10 \mathrm{~min}$ in $100 \% \mathrm{HNO}_{3}$ at $95^{\circ} \mathrm{C}$ ). A dehydration bake on the hotplate $\left(120^{\circ} \mathrm{C}, 5 \mathrm{~min}\right)$ was applied on the wafers before priming with HMDS. Spin-coating of the positive photoresist Olin 907 was done at $4000 \mathrm{rpm}$ for 20 s. A softbake of the resist $\left(95^{\circ} \mathrm{C}, 60 \mathrm{~s}\right)$ was preceding the optical exposure of the resist ( $3 \mathrm{~s}, 12 \mathrm{~mW} \cdot \mathrm{cm}^{-2}$, hard contact with 0.7 bar contact pressure) through a mask. After a post-exposure bake at $120{ }^{\circ} \mathrm{C}$ for $60 \mathrm{~s}$, the resist was developed by mild agitation in OPD 4262 resist developer for $50 \mathrm{~s}$ and the native oxide was removed by etching the wafers in a $1 \%$ aqueous $\mathrm{HF}$ for $60 \mathrm{~s}$. Without delay, the wafers were 
immersed for $60 \mathrm{~s}$ in acetone to strip the resist and $60 \mathrm{~s}$ in isopropanol to clean the wafer, and blown dry by nitrogen before immersion in OPD 4262 resist developer. Etching of the outline structures into the silicon wafer was done for 28 min to result in etch depth of about $100 \mathrm{~nm}$.

FIB milling in the photonic section of the waveguides was carried out with a stateof-the-art NOVA600 FIB dual-beam writer (FIE Company). Photonic designs were made in CorelDraw12 $(d=214 \mathrm{~nm}, a=390 \mathrm{~nm})$, and exported as a 240 dpi tiff-file (at this resolution, the photonic structures just fit in the 12-bit patterning field of the FIB resulting in maximum milling resolution). Conversion of the tiff-file into this patterning field was done in LabVIEW6 in order to translate the pixel information into coordinates for the stream file.

FIB settings on the NOVA600 dual-beam FIB writer: $30 \mathrm{kV}$ accelerating voltage, $93 \mathrm{pA}$ beam current, accumulative dwell time is $\sim 1 \mathrm{~s}$. The offset in the position of the stream file pattern with the actual milling position in the patterning field was $50 \mathrm{~nm}$ in $\mathrm{x}$-direction and $150 \mathrm{~nm}$ in $\mathrm{y}$-direction and the correct magnification was 5400. At this magnification, the field of view (FOV) is $47 \mu \mathrm{m}$ resulting in a digital-to-analog converter $(\mathrm{DAC})$ value of $\sim 11.5 \mathrm{~nm}\left(=\mathrm{FOV} / 2^{12}\right)$. The $\mathrm{DAC}$ value is the limiting factor with respect to the maximum beam current setting as accurate milling requires a reasonable beam overlap. A beam current of $93 \mathrm{pA}$ was chosen for the ion beam milling, as this setting still let us to use practical mill times in combination with a good beam overlap ( 50\%, as the beam diameter was $22.4 \mathrm{~nm}$ at $93 \mathrm{pA})$. To eliminate the effect of stage drift during on the final definition of the photonic elements, the software was set to execute a dynamic drift correction (DDC) every $40 \mathrm{~s}$ on a reference feature $\left(9 \times 9 \mu \mathrm{m}^{2}\right.$ frame $)$ within the FOV. DDC locks the position of the reference feature prior to ion beam milling and corrects for differences in its position due to stage drift. At these current settings, the time for milling a $22 \mu \mathrm{m}$ long $2 \mathrm{D} \mathrm{PhCWG}$ $100 \mathrm{~nm}$ deep into silicon was set by calculation:

mill time $=\frac{\text { number of elements } \times \text { volume per element }}{\text { beam current } \times \text { sputter rate }}=\frac{630 \times 3.6 \cdot 10^{-3} \mu \mathrm{m}^{3}}{93 \cdot 10^{-12} \mathrm{C} \cdot \mathrm{s}^{-1} \times 0.18 \cdot 10^{9} \mu \mathrm{m}^{3} \cdot \mathrm{C}^{-1}}=136 \mathrm{~s}$ 
Finally, the as-fabricated molds were cleaned in a standard cleaning procedure and put on the hot plate at $120{ }^{\circ} \mathrm{C}$ for $5 \mathrm{~min}$ for dehydration prior to application of $1 \mathrm{H}, 1 \mathrm{H}, 2 \mathrm{H}, 2 \mathrm{H}$-perfluorodecyltrichlorosilane to the molds by gas phase deposition for $60 \mathrm{~min}$ in a desiccator under vacuum.

Substrate preparation: Prior to processing the SOI wafers, the native oxide was removed in 1\% $\mathrm{HF}$ for $60 \mathrm{~s}$. Afterwards, a very thin layer of stoichiometric silicon nitride $\left(\mathrm{Si}_{3} \mathrm{~N}_{4}, 14 \mathrm{~nm}\right)$ was deposited by low-pressure chemical vapor deposition (LPCVD, Tempress LPCVD furnace: $70 \mathrm{sccm} \mathrm{SiH}_{2} \mathrm{Cl}_{2}, 18 \mathrm{sccm} \mathrm{NH}_{3}, 850{ }^{\circ} \mathrm{C}$, $200 \mathrm{mTorr}, 150 \mathrm{~s}$ deposition time) with minimal delay. On top of this layer, a very thin layer of LPCVD-SiO ${ }_{2}(12 \mathrm{~nm}$ ) was deposited by LPCVD (Tempress LPCVD furnace: $50 \mathrm{sccm}$ TEOS, $700{ }^{\circ} \mathrm{C}, 400 \mathrm{mTorr}$, bubbler at $40{ }^{\circ} \mathrm{C}, 90 \mathrm{~s}$ deposition time). After the LPCVD runs, the wafers were annealed under dry nitrogen at $800^{\circ} \mathrm{C}$ for $60 \mathrm{~min}$ in the furnace (Tempress, dry oxidation furnace) with minimal delay and cut into smaller substrates $\left(18 \times 18 \mathrm{~mm}^{2}\right)$ for further processing using the Disco dicing saw (model: DAD-321, NBC- $Z$ dicing saw (Disco), spindle at $30000 \mathrm{rpm}$, sawing speed at $\left.1 \mathrm{~mm} \cdot \mathrm{s}^{-1}\right)$. Prior to cutting, a thick $(\sim 3 \mu \mathrm{m})$ layer of photoresist (Olin 907) was applied on the wafer by spin-coating to protect it from damage from particles during sawing. After cutting, the substrates were cleaned using the standard cleanroom cleaning procedure.

Nanoimprint lithography: Polymer solutions were applied onto the substrates by filtering through a syringe filter with a $0.2 \mu \mathrm{m}$ PTFE membrane (Acrodisc CR $25 \mathrm{~mm}$ ). Spin-coating was done at 2000-4000 rpm for $30 \mathrm{~s}$ in order to obtain polymer layers of about $\sim 110 \mathrm{~nm}$ thickness, followed by a prebake at $100-120^{\circ} \mathrm{C}$ for $10 \mathrm{~min}$ to remove residual solvent. For imprinting the mold pattern into a thermoplastic polymer (PMMA, $\mathrm{M}_{\mathrm{w}}=996 \mathrm{kD}$ ), the mold and substrate were placed in between the plates of a commercial hydraulic press (Specac, 15 tons manual press equipped with electrical heating plates and a temperature controller) and heated to the imprint temperature of $180{ }^{\circ} \mathrm{C}$ (heating rate $\sim 30^{\circ} \mathrm{C} \cdot \mathrm{min}^{-1}$ ). Upon reaching this imprint temperature, an imprint pressure of 50 bar was applied to pattern the PMMA layer. After 2 min imprinting at $180^{\circ} \mathrm{C}$ and $50 \mathrm{bar}$, the stack was cooled (cooling rate $\sim 25^{\circ} \mathrm{C} \cdot \mathrm{min}^{-1}$ ) to $106{ }^{\circ} \mathrm{C}$ prior to pressure reduction and manual separation of the mold from the 
polymer substrate with a scalpel. The total process cycle (i.e., heating, imprinting, and cooling) took about $7.5 \mathrm{~min}$.

The residual polymer layer was removed by low-pressure $\mathrm{O}_{2}$ plasma $(\mathrm{Plasmatherm}$ SLR 770). Of fundamental importance to the etch uniformity was the conditioning of the plasma etcher after a high-power plasma cleaning procedure $(20 \mathrm{~m}$ Torr, $800 \mathrm{~W}$ $\mathrm{ICP}$ power, $20 \mathrm{~W} \mathrm{CCP}$ power, $40 \mathrm{sccm} \mathrm{O}$, $40 \mathrm{sccm} \mathrm{Ar}, 25^{\circ} \mathrm{C}$ ). Conditioning was done by etching a thick PMMA layer on silicon for 10 min using similar conditions for etching of the residual layer. The $\mathrm{O}_{2} \mathrm{RIE}$ conditions $\left(5 \mathrm{~m}\right.$ Torr, $5 \mathrm{~W}, 20 \mathrm{sccm} \mathrm{O}_{2}$, $\left.25^{\circ} \mathrm{C}\right)$ were chosen in order to achieve directional etching $(\mathrm{P}<10 \mathrm{~m}$ Torr $)$ that is nondamaging for the polymer structures (low CCP power). At these settings the PMMA etch rate was $8 \mathrm{~nm} \cdot \mathrm{min}^{-1}$, resulting in etch times of $2.5 \mathrm{~min}$.

Local oxidation of silicon (LOCOS): The PMMA imprint patterns were transferred into the $\mathrm{LPCVD}-\mathrm{SiO}_{2}$ layer by etching in a $1 \% \mathrm{HF}$ solution for $45 \mathrm{~s}$. After removal of the PMMA from the substrates in acetone/isopropanol, the $\mathrm{Si}_{3} \mathrm{~N}_{4}$ layer was patterned by etching in hot $\mathrm{H}_{3} \mathrm{PO}_{4}$ of $180{ }^{\circ} \mathrm{C}$ for $5 \mathrm{~min}$. After removal of the $\mathrm{LPCVD}-\mathrm{SiO}_{2}$ mask in a $1 \% \mathrm{HF}$ solution for $2 \mathrm{~min}$, the silicon was locally oxidized in a dry oxidation furnace (Tempress, dry oxidation furnace, atmospheric pressure, $4000 \mathrm{sccm} \mathrm{O}_{2}$, $950{ }^{\circ} \mathrm{C}, 12 \mathrm{~min}$ ) using the $\mathrm{Si}_{3} \mathrm{~N}_{4}$ as the oxidation mask. Removal of the $\mathrm{Si}_{3} \mathrm{~N}_{4}$ mask in $\mathrm{H}_{3} \mathrm{PO}_{4}$ at $180{ }^{\circ} \mathrm{C}$ for 7 min was done just before the cryogenic etching of the silicon to prevent the formation of native $\mathrm{SiO}_{2}$.

Cryogenic reactive ion etching of silicon: Etching of the final 2D PhCWGs into the top silicon layer of the SOI substrates were carried out on a Plasmalab 100 plus system from Oxford Instruments. Prior to mounting the SOI substrate onto the substrate holder (= standard 4-inch p-type silicon wafer), the native oxide was removed in $1 \% \mathrm{HF}$ for $60 \mathrm{~s}$ to assure close to $100 \%$ silicon loading. Mounting of the SOI substrate was done onto the substrate holder with minimal delay using vacuum grease on the backside of the substrate. After loading the wafer plus substrate holder into the reactor, the holding time for etching was set to $2 \mathrm{~min}$ for the system to equilibrate the temperature of the substrate. Parameter settings for the anisotropic etching of the final waveguide structures are: 10 mTorr, $550 \mathrm{~W}$ ICP power, $12 \mathrm{~W}$ CCP power, $34 \mathrm{sccm} \mathrm{SF} 6,-120^{\circ} \mathrm{C}$. At these settings, the silicon etch rate is about $600 \mathrm{~nm} \cdot \mathrm{min}^{-1}$ 
and the selectivity of silicon over thermal $\mathrm{SiO}_{2}$ is about 50. Typical etch times range from $30-40 \mathrm{~s}$.

Characterization: Layer thicknesses on silicon were determined with a Plasmos SD 2002 ellipsometer operating at a wavelength of $632.8 \mathrm{~nm}$. Step height measurements of the silicon molds were carried out with a Sloan Dektak II surface profiler. Highresolution SEM imaging was carried out with a LEO Gemini 1550 FEG-SEM operating in the INLENS mode for secondary electron detection. PSTM measurements were carried out with a Phantom setup, consisting of a scanning nearfield optical microscope and a Mach-Zehnder interferometer, at the Amolf institute.

\subsection{References and notes}

[1] E. Yablonovitch, Phys. Rev. Lett. 1987, 58, 2059.

[2] S. John, Phys. Rev. Lett. 1987, 58, 2486.

[3] J. D. Joannopoulos, P. R. Villeneuve, S. Fan, Nature 1997, 386, 143

[4] J. D. Joannopoulos, R. D. Meade, J. N. Winn, Photonic crystals - Molding the flow of light, Princeton University Press, Princeton, New Jersey, USA 1995.

[5] O. Painter, R. K. Lee, A. Scherer, A. Yariv, J. D. O’Brien, P. D. Dapkus, I. Kim, Science 1999, $284,1819$.

[6] T. F. Krauss, R. M. De la Rue, S. Brand, Nature 1996, 383, 699.

[7] M. Notomi, A. Shinya, K. Yamada, J. Takahashi, C. Takahashi, I. Yokohama, IEEE J. Quant. Electron. 2002, 38, 736.

[8] W. Bogaerts, R. Baets, P. Dumon, V. Wiaux, S. Beckx, D. Taillaert, B. Luyssaert, J. van Campenhout, P. Bienstman, D. van Thourhout, J. Lightwave Technol. 2005, 23, 401.

[9] SOI is also a suitable template for potential telecommunication applications owing to its spectral properties in the $1.5 \mu \mathrm{m}$ wavelength range.

[10] S. Y. Chou, P. R. Krauss, P. J. Renstrom, P. J. Science 1996, 272, 85.

[11] (a) H. Schift, S. Park, B. Jung, C.-G. Choi, C.-S. Kee, S.-P. Han, K.-B. Yoon, J. Gobrecht, Nanotech. 2005, 16, S261. b) C.-G. Choi, C.-S. Kee, H. Schift, Curr. Appl. Phys. 2006, 6S1, e8.

[12] (a) M. Belotti, J. Torres, E. Roy, A. Pépin, D. Gerace, L. C. Andreani, M. Galli, Y. Chen, Microelectron. Eng. 2006, 83, 1773. (b) M. Belotti, J. Torres, E. Roy, A. Pépin, D. Gerace, L. C. Andreani, M. Galli, Y. Chen, J. Appl. Phys. 2006, 99, 024309. (c) M. Belotti, M. Galli, D. Bajoni, L. C. Andreani, G. Guizzetti, D. Decanini, Y. Chen, Microelectron. Eng. 2004, 73-74, 405. 
[13] P. I. Borel, B. Bilenberg, L. H. Frandsen, T. Nielsen, J. Fage-Pedersen, A. V. Lavrinenko, J. S. Jensen, O. Sigmund, A. Kristensen, Opt. Express 2007, 15, 1261.

[14] (a) B. Faircloth, H. Rohrs, R. Tiberio, R. Ruoff, R. R. Krchnavek, J. Vac. Sci. Technol. B 2000, 18, 1866. (b) P. Carlberg, M. Graczyk, E.-L. Sarwe, I. Maximov, M. Beck, L. Montelius, Microelectron. Eng. 2003, 67-68, 203. (c) K. Nakamatsu, K. Watanabe, K. Tone, T. Katase, W. Hattori, Y. Ochiai, T. Matsuo, M. Sasago, H. Namatsu, M. Komuro, S. Matsui, Jpn. J. Appl. Phys. 2004, 43, 4050.

[15] (a) A. Lebib, Y. Chen, F. Carcenac, E. Cambril, L. Manin, L. Couraud, H. Launois, Microelectron. Eng. 2000, 53, 175. (b) S. H. Kim, H. Hiroshima, S. Inoue, Y. Kurashimma, M. Komuro, J. Vac. Sci. Technol. B 2003, 21, 3144. (c) J. Shi, C. Peroz, D. Peyrade, J. Salari, M. Belotti, W. H. Huang, Y. Chen, Microelectron. Eng. 2006, 83, 1664.

[16] E. Kooi, The Invention of LOCOS - IEEE Case Histories of Achievement in Science and Technology, Vol. 1, IEEE, NY 1991, pp. 101-128.

[17] http://www.brl.ntt.co.jp

[18] K. Yamada, H. Morita, A. Shinya, M. Notomi, Opt. Commun. 2001, 198, 395.

[19] (a) M. Notomi, A. Shinya, K. Yamada, J. Takahashi, C. Takahashi, I. Yokohama, Electron. Lett. 2001, 37, 293. (b) M. Notomi, A. Shinya, K. Yamada, J. Takahashi, C. Takahashi, I. Yokohama, IEEE J. Quant. Electron. 2002, 38, 736.

[20] K. Yamada, M. Notomi, A. Shinya, C. Takahashi, J. Takahashi, H. Morita, Electron. Lett. $2002,38,74$.

[21] S. G. Johnson, J. D. Joannopoulos, Opt. Express 2001, 8, 173.

[22] http://wwwhome.math.utwente.nl/ hammerm/oms.html (accessed at 05-06-2008)

[23] T. Baba, A. Motegi, T. Awai, N. Fukaya, Y. Watanabe, A. Sakai, IEEE J. Quant. Electron. 2002, 38, 743.

[24] J. Haneveld, H. V. Jansen, E. Berenschot, N. Tas, M. Elwenspoek, J. Micromech. Microeng. 2003, 13, S62.

[25] At room temperature, a silicon etch rate in the $\langle 110\rangle$ direction, i.e., the direction perpendicular to the wafer surface of the $\langle 110\rangle$ silicon wafer, of $3.7 \mathrm{~nm} \cdot \mathrm{min}^{-1}$ was found along with an excellent etch uniformity $( \pm 1 \mathrm{~nm})$ over the entire 4-inch wafer and a selectivity of (at least) 250 with respect to $\mathrm{SiO}_{2}$ as the etch mask.

[26] Feature dimension analysis of the diameter $(d)$ and periodicity $(a)$ was done by statistical calculation of about 20 measurements from the high-resolution SEM images. Each feature was fit by a circle in order to determine the feature diameter. The periodicity was taken as the distance of the centers from two adjoining features.

[27] At the perimeter of each circular feature the ion beam is milling with $25 \%$ of the current beam diameter $(\sim 6 \mathrm{~nm})$ outside the feature region 
[28] The deviation between the design diameter and the actual diameter therefore is a result of the accuracy of the milling process. The cause of the deviation could reside in the current beam overlap of the milling process and the feature definition in the stream file. At exporting the vector design in CorelDraw12 into a 240 dpi tiff-file, the program approximates the circular elements into an integer number of dots. At 240 dpi (i.e., 94 dots per $\mathrm{cm}$ ), the diameter of each element consists of 19-20 dots. Theoretically, the resulting diameter of the circular elements after translation into a stream file is in the range of 218-230 nm (number of dots $\times$ DAC value). See Experimental (Section 7.4) for further details.

[29] H. Lim, Y. Lee, S. Han, J. Cho, K.-J. Kim, J. Vac. Sci. Technol. A 2001, 19, 1490.

[30] A. P. Milenin, C. Jamois, T. Geppert, U. Gösele, R. B. Wehrspohn, Microelectron. Eng. 2005, 81,15 .

[31] H. V. Jansen, M. J. de Boer, R. Wiegerink, N. Tas, E. Smulders, C. Neagu, M. Elwenspoek, Microelectron. Eng. 1997, 35, 45.

[32] M. D. Austin, H. Ge, W. Wu, M. Li, Z. Yu, D. Wasserman, S. A. Lyon, S. Y. Chou, Appl. Phys. Lett. 2004, 84, 5299.

[33] a) H. Gersen, T. J. Karle, R. J. P. Engelen, W. Bogaerts, J. P. Korterik, N. F. van Hulst, T. F. Krauss, L. Kuipers, Phys. Rev. Lett. 2005, 94, 073903. b) H. Gersen, T. J. Karle, R. J. P. Engelen, W. Bogaerts, J. P. Korterik, N. F. van Hulst, T. F. Krauss, L. Kuipers, Phys. Rev. Lett. 2005, 94, 123901.

[34] For a topical review on near-field characterization of PhCWGs: S. I. Bozhevolnyi, L. Kuipers, Semicond. Sci. Technol. 2006, 21, R1.

[35] http://www.atecorp.com/equipment/hp/8168f.htm (accessed at 05-06-2008)

[36] http://www.corningcablesystems.com

[37] V. S. Volkov, S. I. Bozhevolnyi, L. H. Frandsen, M. Kristensen, Nano Lett. 2007, 7, 2341.

[38] C. Peeters, E. Flück, M. L. M. Balistreri, A. M. Otter, L. Kuipers, N. F. van Hulst, Appl. Phys. Lett. 2000, 77, 142.

[39] This is a characteristic feature of Bloch modes - general wave functions with a periodic amplitude modulation that coincides with the periodic modulation of the potential (in this case, the dielectric constant). See C. Kittel, Introduction to Solid State Physics, 8th edition, Wiley, NY 2005.

[40] M. L. M. Balistreri, D. J. W. Klunder, F. C. Blom, A. Driessen, H. J. W. M. Hoekstra, J. P. Korterik, L. Kuipers, N. F. van Hulst, N. F. Opt. Lett. 1999, 24, 1829.

[41] R. J. P. Engelen, Y. Sugimoto, H. Gersen, N. Ikeda, K. Asakawa, L. Kuipers, Nature Physics 2007, 3, 401. 
[42] The propagation losses for light of TE polarization were determined at different excitation wavelengths from a linear fit of the intensity profiles assuming that the interaction between the probe and the waveguide remains constant over the entire scan area.

[43] Y. Tanaka, T. Asano, R. Hatsuta, S. Noda, J. Lightwave Technol. 2004, 22, 2787.

[44] R. J. P. Engelen, T. J. Karle, H. Gersen, J. P. Korterik, T. F. Krauss, L. Kuipers, N. F. van Hulst, Opt. Express 2005, 13, 4457. 


\section{Summary}

This thesis contributes to the continuous development of patterning strategies in several different areas of unconventional nanofabrication, from the transfer of molecules into periodic patterns onto surfaces (Chapter 3), to the structuring of thermoplastic polymers into functional templates (Chapters 4 and 5), and towards the fabrication of functional nanophotonic devices such as two-dimensional photonic crystal waveguides (2D PhCWGs) (Chapters 6 and 7). The advancements shown in these chapters have been the result of incorporating several potential patterning strategies into existing nanofabrication techniques. These advancements include the precise control over the pattern stability of molecular patterns in microcontact printing $(\mu \mathrm{CP})$ and dip-pen nanolithography (DPN) by the use of multivalent supramolecular interactions of functional molecules with receptor surfaces (i.e., molecular printboards), the facile generation of high-resolution templates in (general) lithography by the use of capillary forces in the molding of thin polymer films at high annealing temperatures, and the accurate pattern transfer in nanoimprint lithography (NIL) by the use of local oxidation of silicon (LOCOS) in combination with ultrathin transfer layers to convert resist patterns into inverse patterns of silicon oxide $\left(\mathrm{SiO}_{2}\right)$. 
An extensive overview of the field of unconventional nanofabrication (from 1995 to mid-2008) with a focus on significant technological advancements has been presented in Chapter 2. A differentiation in the current set of nanofabrication techniques was made between pattern generation and pattern replication, as these represent two distinct patterning concepts. Special attention was given to contributions in the field of unconventional nanofabrication that result in the generation and/or replication of photonic nanostructures. The results throughout this thesis elaborate on several patterning strategies shown in Chapter 2 to further advance the patterning capability of current nanofabrication techniques in terms of the applicability to structure different materials, transfer fidelity, or feature resolution.

Chapter 3 describes the selective transfer of adamantyl-functionalized guest molecules to $\beta$-cyclodextrin $(\beta-\mathrm{CD})$ receptors on surfaces by means of conformal contact with elastomeric stamps or by scanning across the surface with atomic force microscopy (AFM) tips. The degree of guest deposition by transfer of elastomeric poly(dimethylsiloxane) (PDMS) stamps was systematically studied by $\mathrm{X}$-ray photoelectron spectroscopy (XPS), illustrating (in case of a divalent guest) a significant decrease from $\sim 5$ monolayers of guest molecules after transfer down to 1-2 monolayers and even to submonolayer coverages after rinsing with $50 \mathrm{mM} \mathrm{NaCl}$ and 1-10 $\mathrm{mM}$ $\beta-\mathrm{CD}$ solutions, respectively. Additionally, all kinetic stabilities were observed in additional printing and rinsing experiments using monovalent, divalent and multivalent guests by contact-mode AFM imaging, from fast removal during rinsing with water (in case of monovalent guests) to complete stability during rinsing with competitive $\beta-\mathrm{CD}$ solutions (in case of multivalent guests). The tunable pattern stability in combination with the absence of nonspecific interactions, as shown by the complete removal of multivalent guests on non-receptor monolayers, are an explicit demonstration of the concept of multivalency and moreover of the applicability of the molecular printboard in nanofabrication by supramolecular $\mu \mathrm{CP}$ and DPN. This applicability was furthermore supported by the successful electroless deposition (ELD) of copper on top of patterns of catalytically active nanoparticles and the fabrication of molecular patterns at a resolution of $60 \mathrm{~nm}$ by DPN.

Chapter 4 presents the successful combination of capillary force lithography (CFL) and replica molding (REM) to generate second-generation high-resolution stamps for 
soft lithography. Several measures were taken to improve the overall performance of CFL in patterning thin polymer films over large areas, including the use of thin ( $1 \mathrm{~mm}$ thick) PDMS stamps and pre-equilibration of the stamps and substrates to the annealing temperature before conformal contact. Additionally, the process of capillary kinetics during CFL of thin layers of poly(methylmethacrylate) (PMMA) was examined as a function of the initial polymer thickness by AFM analysis, illustrating that some control over the lateral feature dimensions of the resulting polymer templates was feasible in the range of several hundreds of nanometers. The highresolution templates were resistant to the REM conditions, as there was no evidence of noticeable degradation or damage on the AFM scale after generating more than 10 PDMS copies. The applicability of these second-generation stamps in soft lithography was illustrated in a series of different $\mu \mathrm{CP}$ experiments of alkanethiols on gold and successive wet-chemical etching of the gold to generate metal patterns at a resolution of $\sim 125 \mathrm{~nm}$. The accompanying discrepancy between the lateral dimensions of the metal features and the indentations present on the stamp was not further investigated, but is most probably the result of ink diffusion and deformation of the stamp during printing. By optimization of the etch conditions using the experimental black silicon method (BSM), nanochannels with vertical sidewalls were fabricated using the resulting metal patterns as secondary etch masks in the RIE of the silicon substrate.

Chapter 5 describes two additional applications for the high-resolution templates, specifically, as masters in NIL and as etch masks in the wet-chemical etching of metals. For the first time, a UV-curable polymer ( $\mathrm{mr}-\mathrm{L}$ 6000) was implemented in $\mathrm{CFL}$ in order to meet the stringent requirements of high temperature and pressure stability for NIL molds. The exposure dose dependence of the stability of the templates during UV curing was studied in detail by AFM analysis to determine the critical exposure dose for high-fidelity crosslinking. An additional long exposure and hard bake was carried out on the polymer templates to further increase the mechanical and thermal stability, as shown by the perfect response in durability test for tens of imprints. The applicability of these templates in NIL was shown by the fabrication of metal patterns at a resolution of $\sim 150 \mathrm{~nm}$ by metal lift-off and by wet-chemical etching of the underlying metal layer. The transfer of PDMS residues during CFL 
processing was unambiguously proven by XPS analysis and was found (in the case of PMMA and low-modulus stamps) to severely compromise the processability of the polymer templates in applications that necessitate the removal of the residual layer by $\mathrm{O}_{2}$ plasma. The use of poly(styrene) (PS) and longer curing of the PDMS stamps were effective measures to increase the processability of the polymer templates, as shown by the use of these templates as etch masks in the wet-chemical etching of the underlying metal layer. Additionally, the strategy of over-etching was shown as a means to control the reduction of the lateral dimensions of the metal features to a resolution of $\sim 80 \mathrm{~nm}$.

Chapter 6 describes in detail the development of a novel pattern inversion strategy by incorporation of LOCOS in NIL processing. Proper design rules were set in order to convert resist patterns into inverse patterns of $\mathrm{SiO}_{2}$ with high transfer accuracy, resulting in a set of two ultrathin transfer layers by optimization of the etch selectivities and layer thicknesses. High-temperature annealing of the set of transfer layers was found as a means to engineer the etch rates and therefore the etch selectivities of this system. The partial conversion of silicon nitride $\left(\mathrm{Si}_{3} \mathrm{~N}_{4}\right)$ in LOCOS compromises to some extent the processability, as shown by the large reduction of the initial etch rate in the first two minutes of wet-chemical etching of the $\mathrm{Si}_{3} \mathrm{~N}_{4}$ layer. For the resulting ultrathin $(\sim 10 \mathrm{~nm})$ etch masks of thermal $\mathrm{SiO}_{2}$, several guidelines were shown to systematically locate the etch parameters for etching silicon at high anisotropy and high etch selectivity.

Chapter 7 presents the fabrication and successful replication of $2 \mathrm{D} \mathrm{PhCWG}$ on silicon-on-insulator substrates and elaborates on the LOCOS inversion and anisotropic etch strategies shown in Chapter 6. After the fast prototyping of the necessary master by combining focused ion-beam and conventional lithography, 2D PhCWGs were accurately replicated as shown by the very high accuracy $(<4 \mathrm{~nm}$ deviation in lateral feature dimensions) and feature definition of the features using the novel inverse imprinting procedure. Excellent guiding properties at wavelengths in the telecommunications range was observed by local examination of the resulting 2D PhCWGs by photon scanning tunneling microscopy (PSTM), yielding propagation losses down to $36 \mathrm{~dB} \cdot \mathrm{mm}^{-1}$. The high quality of the $2 \mathrm{D} \mathrm{PhCWG}$ was moreover supported by the absence of high amplitude spikes (from the optical field 
distribution image), and the minimal amount of light propagating in the backward direction (from the photonic band diagram).

Collectively, the results in this thesis illustrate the diversity and potential of unconventional nanofabrication in the patterning of matter on the sub-100 $\mathrm{nm}$ scale. The work on molecular printboards demonstrates the applicability of supramolecular interactions in $\mu \mathrm{CP}$ and DPN in the patterning of molecular patterns with tunable kinetic stability. Especially the compatibility of this patterning strategy with DPN will facilitate the development of future detection assays by the immobilization of (bio)molecules on molecular printboards. The work on CFL and the application of the polymer templates in several lithographic applications demonstrate the potential to structure, in this particular case, metal films at high resolution. The general applicability of edge lithography in the cost-effective generation of (periodic) highresolution features will not only find its use in exploratory research, but ultimately in the fabrication of devices containing nanochannels and/or nanowires. Finally, the work on a novel pattern inversion strategy in the low-cost replication of $2 \mathrm{D}$ PhCWGs by NIL illustrates that unconventional nanofabrication techniques can also offer the transfer accuracy and feature definition of state-of-the-art techniques. This resolution enhancement technique is one of many that will further enforce the position of NIL as a candidate for next-generation lithographic technologies in IC manufacturing. 


\section{Samenvatting}

Dit proefschrift beschrijft de continue ontwikkeling van strategieën op een aantal verschillende gebieden van niet-conventionele nanofabricage. Van de overdracht van moleculen in periodieke patronen op oppervlakken (hoofdstuk 3) en het structureren van thermoplastische polymeren in functionele sjablonen (hoofdstukken 4 en 5) tot de vervaardiging van functionele nanofotonische structuren, zoals twee-dimensionale golfgeleiders op basis van fotonische kristallen (2D PhCWGs) (hoofdstukken 6 en 7). De vorderingen die in deze hoofdstukken worden beschreven zijn het resultaat geweest van het incorporeren van een antal strategieën in bestaande nanofabricagetechnieken. Deze vorderingen omvatten de nauwkeurige controle over de stabiliteit van moleculaire patronen met microcontactdruk $(\mu \mathrm{CP})$ en dip-pen nanolithografie (DPN) door het gebruik van multivalente supramoleculaire interacties van functionele moleculen met receptoroppervlakken (moleculaire printplaten). Daarnaast het gemakkelijk genereren van hoge-resolutie-sjablonen voor lithografie (in het algemeen) door het gebruik van capillaire krachten in het patroneren van dunne polymeerfilms bij hoge verhardingstemperaturen. Tenslotte het accuraat overdragen van patronen via nanoimprintlithografie (NIL) door lokale oxidatie van silicium (LOCOS) te 
combineren met uiterst dunne overdrachtslagen om patronen in polymeren te inverteren in silicium oxide $\left(\mathrm{SiO}_{2}\right)$.

Een uitgebreid overzicht van het gebied van niet-conventionele nanofabricage (vanaf 1995 tot medio 2008), met de nadruk op significante technologische vorderingen, is beschreven in hoofdstuk 2. De huidige nanofabricagetechnieken zijn onderverdeeld in het genereren en repliceren van patronen, aangezien dit twee verschillende concepten zijn. Speciale aandacht is besteed aan bijdragen in de nietconventionele nanofabricage gericht op de vervaardiging en/of reproductie van fotonische nanostructuren.

De resultaten beschreven in dit proefschrift borduren voort op een aantal van de fabricagestrategieën uit Hoofdstuk 2 met als doel om de capaciteit in patronering van de huidige nanofabricagetechnieken verder te brengen in termen van toepasbaarheid voor het structureren van verschillende materialen, overdrachtsnauwkeurigheid, en resolutie.

Hoofdstuk 3 beschrijft de selectieve overdracht van adamantyl-gefunctionaliseerde gastmoleculen naar $\beta$-cyclodextrine $(\beta-\mathrm{CD})$-receptoren op oppervlakken, door middel van contact met elastische stempels of door het oppervlak te scannen met een tip van een atomaire-krachtmicroscoop (atomic force microscope, AFM). De mate van depositie door overdracht van elastische poly(dimethylsiloxaan) (PDMS) stempels werd systematisch bestudeerd met Röntgen-fotoelectron spectroscopie (X-ray photospectroscopy, XPS). In geval van een divalente gast liet dit een significante daling zien van $\sim 5$ monolagen gastmoleculen, tot 1-2 monolagen en een submonolaag, na het spoelen met oplossingen van respectievelijk $50 \mathrm{mM} \mathrm{NaCl}$ en 1-10 mM $\beta$-CD. Met de AFM in contact-modus werd in additionele experimenten een scala aan kinetische stabiliteiten waargenomen door gebruik te maken van mono-, di- en multivalente gasten. Van snelle verwijdering tijdens het spoelen met water (in geval van monovalente gastmoleculen) tot volledige stabiliteit tijdens het spoelen met competitieve $\beta$-CD-oplossingen (in geval van multivalent gasten). De instelbare patroonstabiliteit is een expliciete demonstratie van multivalentie en de toepasbaarheid van het moleculaire printboard in nanofabricage met supramoleculaire $\mu \mathrm{CP}$ en $\mathrm{DPN}$. Deze toepasbaarheid werd verder gedemonsteerd door de succesvolle autokatalytische 
depositie (ELD) van koper op patronen van katalytisch actieve nanodeeltjes en de vervaardiging van moleculaire patronen met een resolutie van $60 \mathrm{~nm}$ door DPN.

Hoofdstuk 4 beschrijft de succesvolle combinatie van capillaire-krachtlithografie (CFL) en replica-vormgieten (REM) om hoge resolutiestempels van de tweede generatie te produceren voor zachte lithografie. Via een aantal aanpassingen werden de prestaties van CFL voor het patroneren van dunne polymeerfilms over grote oppervlakken verbeterd, met inbegrip van het gebruik van dunne ( 1 mm ) PDMSstempels en het vooraf thermisch equilibreren van de stempels en de substraten. De capillaire kinetiek tijdens CFL van dunne lagen poly(methylmethacrylaat) (PMMA) werd door AFM-analyse onderzocht, als functie van de initiële polymeerdikte. Dit laat een zekere controle zien van de laterale dimensies van de resulterende polymeersjablonen, over een bereik van enkele honderden nanometers. De hogeresolutie-sjablonen zijn bestand tegen de condities van REM, aangezien er geen merkbare degradatie of schade na het produceren van meer dan 10 PDMS-stempels te zien was. De toepasbaarheid van deze tweede-generatie-stempels in zachte lithografie werd geillustreerd in een reeks van e stempel experimenten met alkaanthiolen op goud en het nat-chemisch etsen van het goud, om metaalpatronen met een resolutie van 125 nm te produceren. De discrepantie tussen de laterale dimensies van de metaalpatronen en de patronen op de stempel is niet verder onderzocht, maar is vermoedelijk het resultaat van inktspreiding en vervormingen van de stempel tijdens contact. Door het optimaliseren van de etscondities met de experimentele zwartesilicium-methode (black silicon method, BSM) en door de metaalpatronen te gebruiken als secundair etsmasker, werden nanokanalen met verticale zijwanden gemaakt in het siliciumsubstraat met behulp van reactief ion-etsen (reactive ion etching, RIE).

Hoofdstuk 5 beschrijft twee andere toepassingen voor de hoge-resolutie-sjablonen, namelijk als mal in NIL en als etsmasker in het nat-chemisch etsen van metaallaagjes. Voor het eerst werd een UV-hardend polymeer (mr-L 6000) toegepast in CFL om aan de strenge vereisten van stabiliteit bij hoge temperatuur en druk voor NIL-sjablonen te voldoen. De afhankelijkheid van de stabiliteit van de sjablonen aan de dosis tijdens de blootstelling tijdens het UV-harden werd in detail bestudeerd door AFM-analyse om de kritieke dosis te bepalen voor een accuraat polymerisatieproces. Een extra lange 
UV-blootstelling en uitbakken van de polymeersjablonen mechanische en verhogen de thermische stabiliteit verder, zoals blijkt uit een duurzaamheidstest voor tientallen afdrukken. De toepasbaarheid van deze sjablonen in NIL werd aangetoond door de vervaardiging van metaalpatronen met een resolutie van $\sim 150 \mathrm{~nm}$, door selectieve metaaldepositie alsmede door het nat-chemisch etsen van de onderliggende metaallaag. De overdracht van PDMS-residuen tijdens het CFL-proces, bewezen door XPS-analyse, veroorzaakt in geval van PMMA-films en lage-modulus-PDMSstempels beperkingen in toepassingen die een verwijdering van de residuale laag door $\mathrm{O}_{2}$-plasma vereisen. Het gebruik van poly(styreen) (PS) en het langer uitharden van de PDMS-stempels zijn efficiënte maatregelen om de toepasbaarheid van de polymeersjablonen te verhogen, zoals aangetoond door het gebruik van deze sjablonen als etsmaskers in het nat-chemisch etsen van de onderliggende metaallaag. Als laatste werd een strategie beschreven voor overetsen om de laterale dimensies van metaalpatronen tot een resolutie van $\sim 80 \mathrm{~nm}$ gecontroleerd te reduceren.

Hoofdstuk 6 beschrijft de ontwikkeling van een nieuwe strategie voor patrooninversie door LOCOS te integreren in het NIL-proces. Richtlijnen werden ontwikkeld voor een paar uiterst dunne overdrachtslagen om polymeerpatronen te inverteren in $\mathrm{SiO}_{2}$ door optimalisering van de etsselectiviteit en laagdikte voor een hoge overdrachtsnauwkeurigheid. Deze overdrachtslagen werden bij hoge temperatuur verdicht om de etssnelheden en dus de etsselectiviteit aan te passen. De gedeeltelijke omzetting van siliciumnitride $\left(\mathrm{Si}_{3} \mathrm{~N}_{4}\right)$ in LOCOS compromitteert in zekere mate de verwerkbaarheid, zoals aangetoond door de grote reductie van de initiële etssnelheid in de eerste twee minuten tijdens het nat-chemisch etsen van de $\mathrm{Si}_{3} \mathrm{~N}_{4}-\mathrm{laag}$. Om het uiterst dunne thermisch- $\mathrm{SiO}_{2}$ als etsmasker te kunnen gebruiken zijn er richtlijnen ontwikkeld voor het optimaliseren van de parameters voor het etsen van silicium met hoge anisotropie en hoge selectiviteit.

Hoofdstuk 7 beschrijft de vervaardiging en succesvolle replicatie van 2D PhCWGs op silicium-op-isolator-substraten, door gebruik te maken van de LOCOS-inversieprocedure en de strategie voor anisotroop etsen uit Hoofdstuk 6. $\mathrm{Na}$ de fabricage van de noodzakelijke master, door gefocusseerde ionen-bundellithografie (focussed ionbeam, FIB) te combineren met conventionele lithografie, werden 2D PhCWGs nauwkeurig nagemaakt, zoals aangetoond door de zeer hoge nauwkeurigheid (< $4 \mathrm{~nm}$ 
afwijking in de laterale dimensies) en definitie van de patronen met de nieuwe procedure voor patrooninversie. Uitstekende lichtgeleidende eigenschappen met propagatie-verliezen tot $36 \mathrm{~dB} \cdot \mathrm{mm}^{-1}$ werden waargenomen bij golflengtes in het telecommunicatiebereik door de 2D PhCWG lokaal te bestuderen met de fotonraster-tunnelmicroscoop (photon scanning tunneling microscope, PSTM). De uitstekende kwaliteit van 2D PhCWGs werd bovendien ondersteund door de afwezigheid van hoge amplitudepieken (in de ruimtelijke verdeling van het optisch veld) en de minimale hoeveelheid licht dat propageert in de achterwaartse richting (in het fotonisch bandendiagram).

De onderzoeksresultaten beschreven in dit proefschrift illustreren de diversiteit en de potentie van niet-conventionele nanofabricage in het patroneren van materie op de sub-100-nm-schaal. Het werk aan moleculaire printplaten toont de toepasbaarheid van supramoleculaire interacties in $\mu \mathrm{CP}$ en DPN aan in het genereren van moleculaire patronen met variabele kinetische stabiliteit. Het is vooral de compatibiliteit van deze strategie met DPN, die de ontwikkeling van toekomstige analysetechnieken zal stimuleren door de immobilisatie van (bio)moleculen op moleculaire printplaten. Het werk aan CFL en de toepassing van de polymeersjablonen in verscheidene lithografische toepassingen tonen de potentie aan om metaalfilms met hoge resolutie te structureren. De algemene toepasbaarheid van randlithografie in het efficient fabriceren van (periodieke) patronen met hoge resolutie zal uiteindelijk ook belangrijk zijn voor de vervaardiging van apparaten die nanokanalen en/of nanodraden bevatten. Tot slot illustreert het werk an de nieuwe strategie voor patrooninversie met de replicatie van 2D PhCWGs, dat de niet-conventionele nanofabricatie technieken de nauwkeurigheid en de patroondefinitie van de huidige technieken kunnen bieden. Deze techniek om de resolutie te verhogen is één van vele technieken die de kansen voor NIL als kandidaat voor lithografische technologie van de volgende generatie in de IC-fabricage verder zullen versterken. 


\section{Dankwoord}

'Aan alles komt een einde', dus ook aan mijn promotieonderzoek aan de Universiteit Twente. Dit proefschrift is tot stand gekomen in samenwerking met en dankzij de inspanningen van een groot aantal mensen. Graag wil ik van de gelegenheid gebruik maken om op de laatste pagina's van mijn proefschrift een ieder te bedanken die op zijn/haar eigen manier heeft bijgedragen aan de totstandkoming van dit proefschrift. Een aantal mensen wil ik in het bijzonder noemen, zonder wie het onderzoek niet mogelijk was geweest.

Op de eerste plaats wil ik mijn eerste promotor David Reinhoudt bedanken. David, jij hebt mij de mogelijkheden gegeven om mezelf in de SMCT-groep op wetenschappelijk gebied te ontwikkelen. Het was een groot genoegen om voor jou te mogen werken binnen een organisatie als $\mathrm{MESA}^{+}$waarin de faciliteiten nagenoeg onbeperkt zijn. Ik heb naast je wetenschappelijke input vooral je persoonlijke bemoedigingen en je vertrouwen in mij in moeilijke tijden erg kunnen waarderen! Het spreekwoordelijke gezegde 'achter elke succesvolle man staat een sterke vrouw' gaat zeker op voor je vrouw. Leni, erg bedankt voor je persoonlijke inbreng in de SMCTgroep. 
Ik wil mijn grote waardering uitspreken voor mijn tweede promotor, Jurriaan Huskens. Je hebt me met jouw enthousiasme kennis laten maken met nanofabricage en je hebt me op jouw manier alle vrijheid gegeven om in de MnF-groep zelfstandig onderzoek te kunnen verrichten. Ik heb erg veel opgestoken tijdens onze werkbesprekingen en van je aanwijzingen om goede wetenschappelijke artikelen te schrijven. Je betrokkenheid voor mijn persoonlijke situatie als dagelijks begeleider was voor mij erg belangrijk en zal me voor altijd bijblijven!

Het is een voorrecht om Kobus Kuipers als raadgever tijdens je promotieonderzoek te hebben. Je enorme gedrevenheid voor wetenschappelijk onderzoek heeft voor mij erg aanstekelijk gewerkt en je hebt me tijdens werkbesprekingen een aantal keer weten te verrassen met ogenschijnlijk simpele vragen over de processing, waar ik niet direct een antwoord op wist te geven. Als chemisch ingenieur ben ik je erg dankbaar dat je mij als leek in de fysica meermaals de fysische achtergrond van fotonische kristallen hebt willen uitleggen.

Ik ben Gijs Krijnen erg dankbaar dat je me altijd op mijn prioriteiten hebt gewezen en dat je bereidwillig was om het afronden van dit proefschrift onder werktijd toe te staan.

Ik weet nog goed dat ik op aanraden van mijn promotor David contact heb gezocht met de TST-groep voor technische ondersteuning in de cleanroom. Dat is een gouden tip gebleken, want ik heb het uiteindelijk erg getroffen met Meint de Boer, Erwin Berenschot en Henri Jansen. Ik wil jullie in het bijzonder bedanken dat ik altijd met mijn vragen bij jullie terecht kon en daardoor veel ervaring heb opgedaan in processing. Jullie bijdrage is in elk hoofdstuk zichtbaar. Het was jullie humor waar ik even aan moest wennen, maar ik doe inmiddels net zo hard mee en heb erg genoten van de vele grappen!

Een speciaal woord van dank aan het team van de $\mathrm{MESA}^{+}$-cleanroom die dagelijks zorgen voor een goede werkomgeving in de cleanroom: Eddy Ruiter (SEM), Ite-Jan Hoolsema (etsers, LPCVD), Rene Wolf (etsers), Hans Mertens (metaal depositie), Huib van Vossen, Gerrit Boom en Dominique Altpeter (fotolithografie), Peter Linders (maskers), Samantha Ooijman-Geerdink en Marion Nijhuis-Groen (technische ondersteuning en LPCVD) en Gerard Roelofs (hoofd cleanroom). 
Een goede analyse is onmisbaar voor het verrichten van procesoptimalisatie en zonder de hulp van Mária Péter (AFM) en Mark Smithers (SEM) was het mij niet zo goed gelukt. Mária, ik veel respect voor de snelheid en precisie waarmee jij een substraat met een enkele AFM-tip kan afzoeken naar nanostructuren en ik wil je danken dat je ondanks de werkdruk altijd tijd voor mij hebt kunnen vrijmaken. Mark, ik verbaas me bij elke sessie weer hoe jij op de laagste vergroting op de SEM al de nanostructuren kunt zien (hoe doe je dat toch?). Mária en Mark, erg bedankt voor de goede analyses en het meedenken om de processing te verbeteren.

Tijdens mijn promotieonderzoek werd ik meteen opgenomen in een hechte internationale groep als SMCT/MnF. Daar heb ik het erg mee getroffen. Het onderzoek beschreven in hoofdstuk 3 is een deelresultaat van het grootschalige project aan moleculaire printplaten door de samenwerking met Tomasso Auletta, Alart Mulder en Steffen Onclin en generatie genootjes Manon Ludden, Christian Nijhuis en Olga Crespo-Biel. Ik wil jullie allemaal bedanken voor de leuke samenwerking en goede discussies over de moleculaire printplaten.

Aan het onderzoek beschreven in de hoofdstukken 4 en 5 ging een leuke uitdaging vooraf: het opstarten van een antal technieken (CFL, NIL) in de SMCT/MnFgroep. Pascale Maury, bedankt voor de goede samenwerking en het delen van je expertise op het gebied van NIL. Emiel Speets en Albert van den Berg wil ik bedanken voor het uitvoeren van de XPS-analyse op een groot aantal substraten en Gerhard Bleidiessel (MicroResist Technology $\mathrm{GmbH}$ ) voor het doneren van polymeer mr-L 6000.

De optische resultaten beschreven in hoofdstuk 7 zijn te danken aan de inzet van een aantal mensen. Frans Segerink (OS), je bijdrage an het onderzoek is groter geweest dan alleen het genereren van de fotonische structuren met de FIB. Bedankt dat je altijd bereid was om mee te denken aan het onderzoek. Ik wil uit de groep van Kobus Kuipers (NanoOptics, AMOLF) Matteo Burresi en Rob Engelen bedanken voor respectievelijk de metingen met de PSTM en de theoretische berekening van de dispersiecurve van de fotonische structuur. De bijdrage van AMOLF is erg belangrijk geweest voor het slagen van dit deel in mijn promotieonderzoek.

Een aantal vaste steunpunten in de SMCT/MnF/TST-groep wil ik bedanken voor de goede secretariële (Carla van der Ploeg, Izabel Katalanc, Marieke Slotman en Susan 
Janse) en labtechnische ondersteuning (Marcel de Bruine en Richard Egberink) die ik tijdens mijn promotieonderzoek heb mogen ontvangen. Het gaat te ver om iedereen in het SMCT/MnF-laboratorium en de cleanroom op te sommen en het zou niet correct zijn om maar een aantal mensen bij naam te noemen. De goede werksfeer in het laboratorium en de cleanroom hebben zeker bijgedragen aan het eindresultaat!

Ik wil Mark Smithers en Aldrik Velders bedanken voor het zorgvuldig corrigeren van mijn concept proefschrift.

Albert van Eijk heeft mij in de moeilijkste periode van mijn leven van veel goede raadgevingen voorzien om elke keer weer het beste uit mijn persoonlijke situatie te halen. Erg bedankt!

Als laatste wil ik nog een aantal mensen bedanken die een speciale plaats innemen in mijn leven. Lieve ouders, ik heb het jullie de laatste jaren niet altijd even gemakkelijk gemaakt ondanks jullie onvoorwaardelijke steun en het bijspringen in de verzorging van de tweeling. Ik ben jullie erg dankbaar en weet dat jullie er trots op zullen zijn dat jullie zoon gaat promoveren. Dat is grotendeels toch aan jullie te danken! Ik ben Rebecca Zimmerman erg dankbaar dat ze mij een jaar lang heeft geholpen in de verzorging van de tweeling en zal onze persoonlijke gesprekken op moeilijke momenten nooit vergeten! Carsten en Lars, mijn grote kereltjes, jullie zijn niet weg te denken uit mijn leven. Ik ben erg trots op jullie. Het is bewonderenswaardig hoe jullie je in de eerste jaren van jullie leven elke keer weer moeiteloos hebben weten aan te passen aan nieuwe veranderingen. Jullie zijn nog te klein om te begrijpen wat papa tijdens zijn promotieonderzoek heeft gedaan, maar toch wisten jullie in de weekenden (ja, al vanaf 5-6 uur's ochtends!) voor de nodige afleiding te zorgen om op het werk goed te kunnen presteren. Ириша, любовь моя! Я считаю, что мне повезло иметь такую жену как ты и я благодарен твоей безусловной любви, терпению и поддержке во время написания части моей диссертации. Я восхищаюсь твоей способностью видеть лучшее в любой ситуации и обожаю то, как ты заботишься о Карстене и Ларсе, наших семьях и друзьях. Я жду с нетерпением того момента, когда ты окончательно приедешь в Голландию, чтобы жить вместе со мной и близнецами. Навеки твой. 